




\section{Quieting: A Practical Guide 763067

Raymond D. Berendt and Edith L. R. Corliss

Mechanics Division

Institute for Basic Standards

National Bureau of Standards

Washington. D.C. 20231

Morris S. Ojalvo

National Science Foundation

1800 G Street, N.W.

Washington, D.C. 20006

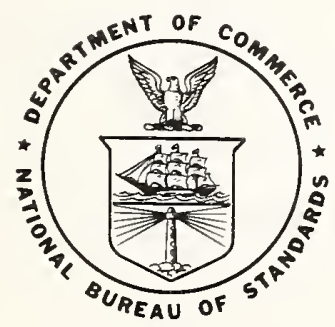

U.S. DEPARTMENT OF COMMERCE, Elliot L. Richardson, Secretary

Dr. Betsy Ancker-Johnson, Assistant Secretary for Science and Technology

U.S. NATIONAL BUREAU OF STANDARDS, Ernest Ambler, Acting Director

Issued July 1976 


\title{
Library of Congress Cataloging in Publication Data
}

Berendt, Raymond D.

Quieting.

(National Bureau of Standards handbook; 119)

Includes index.

Supt. of Dors. no.: C13.11:119

1. Noise control. 2. Soundproofing. I. Corliss, Edith L. R., joint author. II. Ojalvo, Morris S. III. Title. IV. Series: United States National Bureau of Standards. Handbook; 119.

QC100.U565 no. 119 [TD892] 620.2'3

$76-5500$

\section{National Bureau of Standards Handbook 119}

Nat. Bur. Stand. (U.S.), Handb. 119, 163 pages (July 1976)

\author{
CODEN: NBSHAP
}

\section{U.S. GOVERNMENT PRINTING OFFICE \\ WASHINGTON: 1976}

For sale by the Superintendent of Documents, U.S. Government Printing Office, Washington, D.C. 20402 Price $\$ 5.10$ (Add 25 percent for other than U.S. mailing).

Order by SD Catalog No. C13.11:119/Stock No. 003-003-01646-2 
WHAT IS NOISE?

HOW DOES NOISE ORIGINATE?

THE CHARACTERISTICS OF SOUND OR NOISE

Decibel, usually written dB. . . . . . . . . . . . . . . . . . 3

Sound Level Meter . . . . . . . . . . . . . . . . . . . . . 3

A-weighting . . . . . . . . . . . . . . . . . . . . . . . 4

The Notation $\mathrm{dB}(\mathrm{A})$. . . . . . . . . . . . . . . . . . . . . . 6

Frequency and Pitch. . . . . . . . . . . . . . . . . . . . 6

Loudness, Sound Pressure and Intensity . . . . . . . . . . . . . . 6

Phons . . . . . . . . . . . . . . . . . . . . . . . . . . . 7

Pure Tone and Broadband Noise. . . . . . . . . . . . . . . . . . 7

\section{CHAPTER 2}

NOISE AS A HEALTH HAZARD . . . . . . . . . . . . . . . . . . 8

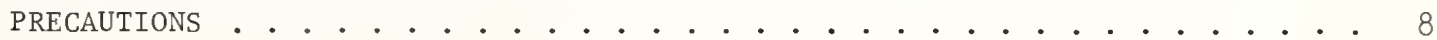

WARNING 非 1 Avoid areas that cause a hearing threshold shift. . . . . . . 8

WARNING 非2 Avoid areas where you must shout to converse . . . . . . . 9 9

WARNING 非3 Avoid areas that cause a tickle sensation in your ears . . . 11

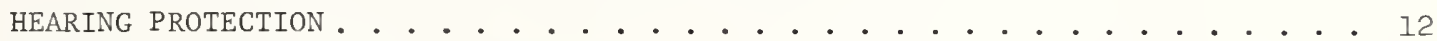

Situations to Avoid. . . . . . . . . . . . . . . . . 12

Signs of Hearing Loss. . . . . . . . . . . . . . . . . . . 12

Noisy Activities That Should Be Limited . . . . . . . . . . . . . 13

HOW TO AVOID RISKING YOUR LIFE AT NOISE LEVELS NOT DAMAGING TO HEARING . . . . 13

Lesson 1 Provide for sounds of emergency vehicles . . . . . . . . . 14

Lesson 2 Take frequent rests in quiet areas . . . . . . . . . . 15

Lesson 3 Avoid startle reactions................ 15

Lesson 4 Control noise at source. . . . . . . . . . . . . . . 15

\section{CHAPTER 3}

BASIC PRINCIPLES OF NOISE REDUCTION . . . . . . . . . . . . . . . 16

SOURCE-PATH-RECEIVER CONCEPT . . . . . . . . . . . . . . . . 16

NOISE CONTROL AT THE SOURCE . . . . . . . . . . . . . . . 16

Select Quiet Equipment ................. 16

REQUIREMENTS FOR QUIET OPERATION . . . . . . . . . . . . . . . . . 17

1. Reduce Impact or Impulsive Forces . . . . . . . . . . . . . . 17

2. Reduce Speed in Machines, and Flow Velocities and Pressures

in Fluid Systems ................. 18

3. Balance Rotating Parts . . . . . . . . . . . . . . 20

4. Reduce Frictional Resistance . . . . . . . . . . . . . . . 20

5. Isolate Vibrating Elements Within the Machine.......... . 23

6. Reduce Radiating Area . . . . . . . . . . . . . . 26 
7. Apply Vibration Damping Materials

8. Reduce Noise Leakage From the Interior of the Appiance. . . . 26

9. Choose Quieter Machinery When Replacing Appliances. . . . * 28

NOISE CONTROL OF THE TRANSMISSION PATH . . . . . . . . . . . . 29

FACTORS AFFECTING TRAVEL OF SOUND . . . . . . . . . . . . . 29

1. Absorption: Soaking Up Sound . . . . . . . . . . . . . 29

2. Sound Reflection: Bouncing of Sound............... . 31

3. Reverberation: Multiple Reflection of Sound............ 32

4. Diffraction: Bending or Squeezing of Sound . . . . . . . . 32

"QUiETING" IN THE PATH . . . . . . . . . . . . . . . . . . 33

1. Separate the Noise Source and Receiver as Much as Possible.... . 33

2. Use Sound Absorbing Materials . . . . . . . . . . . . . 34

3. Use Sound Barriers and Deflectors ............... 35

4. Use Acoustical Lining. . . . . . . . . . . . . . . . . 36

5. Use Mufflers, Silencers or Snubbers . . . . . . . . . . . . 37

6. Use Vibration Isolators and Flexible Couplers . . . . . . . . . 38

7. Use Enclosures . . . . . . . . . . . . . . . 40

PROTECT THE RECEIVER . . . . . . . . . . . . . . . . . . . 40

USE EAR PROTECTORS . . . . . . . . . . . . . . . . . . 40

ALTER WORK SCHEDULE . . . . . . . . . . . . . . . . . 40

IS THERE AN ACOUSTICAL PERFUME? . . . . . . . . . . . . . . . 41

\section{CHAPTER 4}

NOISE CONTROL AT HOME . . . . . . . . . . . . . . . . . . 45

TRANSMISSION OF AIRBORNE AND STRUCTUREBORNE NOISE. . . . . . . . . . . 45 SOUNDING BOARD EFFECT . . . . . . . . . . . . . . . . . . . . 46

FLANKING NOISE TRANSMISSION . . . . . . . . . . . . . . . . . . 47

GENERAL SOLUTIONS FOR NOISE CONTROL IN THE HOME. . . . . . . . . . . . . . . 48

SOUND ABSORPTION VS SOUND INSULATION . . . . . . . . . . . . . . . 50

KITCHEN NOISE . . . . . . . . . . . . . . . . . . 51

INSTALL DOORS . . . . . . . . . . . . . . . . . 52

CONTROL APPLIANCE NOISE. . . . . . . . . . . . . . . 52

1. Vibration Isolate the Appliance from the Floor . . . . . . . . 52

2. Isolate the Appliance from the Wall . . . . . . . . . . . 52

3. Isolate the Appliance from the Cabinet Enclosure. . . . . . . . 53

4. Isolate the Cabinet Enclosure from the Wall . . . . . . . . . 53

5. Isolate Small Appliances . . . . . . . . . . . . . . 53

6. Cushion Impact Noise in the Cabinets . . . . . . . . . . . 54

7. Apply Vibration Damping Material . . . . . . . . . . . . 54

8. Install Acoustical Lining. . . . . . . . . . . . . . . 54

9. Install Exhaust Fans on Rubber Mounts... . . . . . . . . . . . 54

10. Instal1 Acoustic Ceiling . . . . . . . . . . . . . 55

11. Install Carpet or Foam-Backed Tile............ . 55

12. Quieting of Large Appliances . . . . . . . . . . . 55

RECREATION OR PLAYROOMS . . . . . . . . . . . . . . . . . 57

CORRIDORS, HALLS, STAIRCASES. . . . . . . . . . . . . . . . . 58

IMPROVING THE PRIVACY OF YOUR BEDROOM, STUDY OR BATH AREA . . . . . . . . 58

IMPROVING THE SOUND INSULATION OF WALLS . . . . . . . . . . . . . . 60

IMPROVING THE SOUND INSULATION OF FLOOR-CEILING CONSTRUCTIONS . . . . . . 65

CONTROL OF FLOOR SQUEAKING. . . . . . . . . . . . . . . . . . . 66

CONTROL OF STAIR SQUEAKING. . . . . . . . . . . . . . . . . 69 
OF HEATING, VENTILATING AND AIR-CONDITIONING SYSTEM NOISE•

CONTROL OF DUCT NOISE . . . . . . . . . . . . . . . . . . . . • • 74

FURNACE COMBUSTION NOISE . . . . . . . . . . . . . . . . . . . . . . 78

STEAM HEAT NOISE . . . . . . . . . . . . . . . . . . . . . . . 78

NOISE FROM BASEBOARD HEATING SYSTEMS . . . . . . . . . . . . . . . . 78

CONTROL OF PLUMBING NOISE. . . . . . . . . . . . . . . . . . . . . . . . 79

CONTROL OF NOISE FROM RAIN GUTTERS AND SPOUTS. . . . . . . . . . . . . . 85

\section{CHAPTER 5}

HOW TO INSULATE YOUR HOME AGAINST OUTDOOR NOISE . . . . . . . . . . . . . • • 86

Reducing the Intrusion of Neighborhood Noise . . . . . . . . . . . . 86

Reducing the Intrusion of Traffic Noise. . . . . . . . . . . . . . . 87

Reducing the Intrusion of Aircraft Noise. . . . . . . . . . . . . 87

\section{CHAPTER 6}

SELECTING A QUIET HOME OR APARTMENT (or how to avoid making the same

mistake twice) . . . . . . . . . . . . . . . . . . . . . . 9 97

Selecting a Quiet Residential Area . . . . . . . . . . . . . . . . 97

Selecting a Quiet Location Within a Housing or Apartment Development . . . . . 98

Selecting a Quiet Apartment Unit Within the Building . . . . . . . . . .101

\section{CHAPTER 7}

A TROUBLE-SHOOTING GUIDE . . . . . . . . . . . . . . . . . . . . . . 102

ANALYZING THE PROBLEM . . . . . . . . . . . . . . . . . . . . . 102

HOW TO LOOK FOR NOISE LEAKS. . . . . . . . . . . . . . . . . . . . .104

USE YOUR EARS. . . . . . . . . . . . . . . . . . . . . . 104

USE HOME APPLIANCES AS DIAGNOSTIC TOOLS . . . . . . . . . . . . . . . . . 104

COMMON NOISE COMPLAINTS AND LIKELY CAUSES AND SOLUTIONS. . . . . . . . . . . . 107

GENERAL NOISE ABATEMENT TECHNIQUES . . . . . . . . . . . . . . . . . . . 109

INDEXED CATALOG OF SPECIFIC NOISE PROBLEMS AND REMEDIES . . . . . . . . . . . 110

\section{CHAPTER 8}

NOISE CONTROL AT THE OFFICE AND SCHOOL . . . . . . . . . . . . . . . . . 127

NOISE SOURCES . . . . . . . . . . . . . . . . . . . . 127

REDUCING THE NOISE . . . . . . . . . . . . . . . . . . . 127

EXTRANEOUS NOISE . . . . . . . . . . . . . . . . . . . 128

PRIVACY BETWEEN OFFICES . . . . . . . . . . . . . . . . . . . 128

PRIVACY IN OPEN-PLAN OFFICES. . . . . . . . . . . . . . . . . . . . . 129

NOISE CONTROL AT SCHOOL . . . . . . . . . . . . . . . . . . . . 130

CAUSES OF NOISE . . . . . . . . . . . . . . . . . . . . . . . 131

REDUCING CLASSROOM NOISE . . . . . . . . . . . . . . . . . . . 131

NOISE CONTROL IN MULTIPURPOSE ROOMS . . . . . . . . . . . . . . . . 132

\section{CHAPTER 9}

NOISE CONTROL WHILE TRAVELING . . . . . . . . . . . . . . . . . . 133

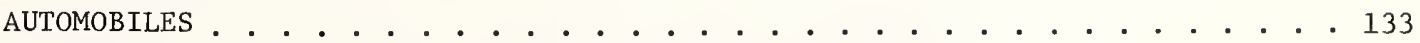

1. Select a "Quiet" Automobile . . . . . . . . . . . . . . 133

2. Drive at Slower Speeds . . . . . . . . . . . . . . . . 133

3. Close Windows for High Speed Driving . . . . . . . . . . . . 133

4. Use Slow Fan Speed . . . . . . . . . . . . . . . . . 133

5. Turn off the Radio..................... . 133

6. Select Quiet Tires . . . . . . . . . . . . . . 134 
7. Balance Car Wheels . . . . . . . . . . . . . 134

8. Reduce Windshield Wiper Noise . . . . . . . . . . . . 134

BUSES AND TRAINS . . . . . . . . . . . . . . . . . . . . . 135

AIRPLANES . . . . . . . . . . . . . . . . . . . . . . 135

SELECTING A "QUIET" MOTEL OR HOTEL . . . . . . . . . . . . . . . 135

SELECTING A "QUIET" ROOM . . . . . . . . . . . . . . . . 137

\section{CHAPTER 10}

POLITICAL AND LEGAL ACTION -- WHEN ALL ELSE FAILS . . . . . . . . . . . 140

INTRODUCTION . . . . . . . . . . . . . . . . . . 140

FEDERAL NOISE REGULATION . . . . . . . . . . . . . . . . . 140

STATE AND LOCAL REGULATION . . . . . . . . . . . . . . . . . 141

CORRECTIVE ACTION PROGRAMS . . . . . . . . . . . . . . . . 141

LOCAL NEIGHBORHOOD NOISE PROBLEMS . . . . . . . . . . . . . . 142

BIBLIOGRAPHY. . . . . . . . . . . . . . . . . . . . . 143

$\operatorname{INDEX}$. . . . . . . . . . . . . . . . . . . . . . 145 

In those days the world teemed; the people multiplied, the world bellowed like a wild bull, and the great god was aroused by the clamour. Enlil heard the clamour and he said to the gods in council, "The uproar of mankind is intolerable and sleep is no longer possible by reason of the babel." So the gods agreed to exterminate mankind.

From "The Epic of Gilgamesh"

Translated by N. K. Sandars

(Penguin Press, 1972)

\section{ABS TRACT}

This guide offers practical solutions for ordinary noise problems that a person is likely to meet. The discussion describes the ways in which sounds are generated, travel to the listener, and affect his hearing and well-being. Recommendations are given for controlling noise at the source and along its path of travel, and for protecting the listener. The guide instructs the reader by way of "Warning Signs" on how to determine whether he is being subjected in his environment to prolonged noise exposures that may prove hazardous to his hearing. Remedies are given for noise problems that a person is likely to find in his home, at work and at school, while traveling, and in the growth and development of his community. The remedies include noise prevention techniques and selection of quiet alternatives to existing noise sources. General principles for selecting quiet appliances are given. Ways of searching for the sources of noise and for determining the paths over which they travel to the listener are described. A detailed index is given for individual noise sources describing specific solutions to the problems they present. General ways of looking for inherently quiet homes and travel accommodations are described. In a final chapter, there are suggestions for enlisting community help where large external sources of noise must be quieted, such as those arising from public utilities and public transportation.

KEY WORDS: Airborne and structure-borne sounds; aircraft noise intrusion; annoyance; appliance noise; health and hearing hazards; household noise; legal and community action; loudness; noise control and abatement; traffic noise. 


\title{
QUIETING
}

\section{A PRACTICAL GUIDE TO NOISE CONTROL}

\author{
Raymond D. Berendt, Edith L. R. Corliss \\ and \\ Morris S. Ojalvo
}

Institute for Basic Standards

National Bureau of Standards

Washington, D. C. 20234

\section{Chapter 1}

\section{INTRODUCTION}

\section{WHAT IS NOISE?}

One humorous definition holds that "Noise is what your neighbor makes." of course, we all make noise ourselves, but we especially resent the intrusion of sounds that we do not choose to hear. Thus, noise may be defined as "unwanted sound." It invades our privacy and disrupts the enjoyment and full use of our surroundings. At worst, it can affect our hearing, performance, and behavior. Indeed, millions of workers risk permanent hearing loss from exposure to noise in their work.

Not only is much of this noise unnecessary, but it is avoidable. Most experts will agree that modern technology for noise control can be applied to reduce much noise to acceptable levels. Often noise arises through thoughtlessness and neglect, and may be remedied by attention to a relatively few basic principles.

The objective of this Guide is to explain and illustrate in a simple manner how many of the commonly encountered noise problems can be alleviated or even eliminated.

HOW DOES NOISE ORIGINATE?

Generally speaking, noise is produced when an object or surface vibrates rapidly enough to generate a pressure wave or disturbance in the surrounding air. Air is a rather springy, elastic substance composed of small particles called molecules. The elasticity is due to the tendency of these molecules to spring back to their original positions of rest whenever they are deflected or displaced by some outside disturbance or force. As a sound wave travels through the air, the pressure fluctuations cause the molecules to vibrate back and forth about their normal positions of rest. In so doing, they bump neighboring molecules, imparting motion to them and then, rebounding back and forth, return to their original positions. The disturbed molecules collide with others in the same manner. The disturbance travels through the air by successive collisions, much as a billiard cue ball will impart motion to a line of billiard balls. Although each of the balls moves with the disturbance, each returns to its original position after collision with the next successive ball; only the end ball, which makes no further collision, moves forward beyond its rest position. In a similar fashion, the vibration imparted to the air travels in a chain-reaction manner from one air molecule to another until it eventually reaches our ears. Colliding molecules produce a pressure increase or a "compression" in the air; molecules rebounding away from each other produce a lower pressure or a "rarefaction". In spite of all this motion, the individual air molecules do not travel far from their original positions; it is only the pressure wave or the compression/rarefaction disturbance that moves outward, like the ripples in a pond into which a pebble is dropped. We hear sounds because our ears respond to the pressure fluctuations that have been set up in the air around us.

A few noises originate directly in the air. The most dramatic is the thunderclap that originates from a discharge of lightning. The electrical forces and the local heating create a sudden burst of overpressure that compresses the air surrounding the path. This pushes the air outward, and the motion persists until the surrounding air particles have been pushed outward by the particles driven by the original stroke. By this time, the column of air has been somewhat over-expanded, a process that cools it and lowers the pressure within it. As a result, some air molecules are sucked back into the column, 
and by the time they have collided with the central part of the column, a pressure rise has been generated that again pushes outward on the column. What started out as a pulse from a single instantaneous heat surge becomes a succession of high-pressure pulses separated by low-pressure pulses, which gradually diminish as the wave motion spreads outward from the column. These we recognize as the compressions and rarefactions in a sound wave.

Another noise that originates directly in the air is turbulence. You can see the swirls of turbulence that your breath generates if you blow into an illuminated column of smoke or dust, such as one illuminated by a beam of sunshine coming through a window. The swirls that you see are larger in diameter and slower in motion than the turbulence you can hear, but have the same general form as the audible turbulence generated at the edge of a jet-engine exhaust or the blades of a high-speed fan. The swirls of turbulence originate whenever a fast flow of fluid goes past a stationary or slowly-moving surface of an obstacle or a fluid.

Most noises are started by the transfer of motion from a vibrating surface to the air lying next to it. If this motion is to be transferred efficiently, the radiating surface must be large compared to the spacing between successive pressure pulses. The pressure pulses travel through the air at the speed of sound, 345 meters/second (1130 feet/second - a little slower than a mile in four seconds). If the pressure can flow from the front to the back of the surface in a very short time, it can restore the neutral condition, because if the front surface of a moving object is compressing the air, the back surface must at the same time be allowing the air behind it to expand.

As far as the listener is concerned, a sonic boom sounds very much like a loud thunderclap. Whereas the lightning generates an initial overpressure by electrical heating, a supersonic plane or the blade tip of a high speed helicopter generates the overpressure by compressing the air in its path faster than the high-pressure disturbance can flow away. This compression is called a "shock wave". Once the sonic boom has been created, the subsequent behavior of the sound is similar to that of thunder.

You can generate relatively high sound levels with the motion of a small surface if there is little opportunity for the air to flow around and equalize the pressure between successive motions, as in the case of striking a kettledrum. There you are changing the pressure in an enclosed volume, and the cancellation from the back surface cannot occur.

Except for our own voices, which reach us primarily through the bony structures in our heads, most sounds reach us through the air. We say they are airborne. However, sounds travel rapidly and with little loss through liquids and solids. As long as these offer an uninterrupted path, they transmit vibrations readily. In liquids and solids the speed of sound is from four to ten times as great as it is in air.

From experience, you know that if you press your ear against a wall in your home or apartment, you can hear noises arising from some distance. You may be able to identify quite clearly your own dishwasher, a distant TV set, or the compressor in the air-conditioner system. Often, when you press your ear against a water pipe, you can hear the sound of running water, perhaps originating at a neighbor's faucet. Some of us have had the childhood experience of listening to the railroad track to hear whether there was a train on the line. These are examples of structure-borne sounds.

A disturbance continues to travel until it dissipates itself and becomes indistinguishable from the random motions of the molecules. We hear the sounds coming from a liquid or solid when they are transferred to our ears as pressure fluctuations if they occur within the range of frequencies to which our ears are sensitive. We do not normally hear the thermal vibrations of molecules because they are too small and too random to generate enough sound, and vibrations occur at frequencies for which our ears are insensitive.

\section{THE CHARACTERISTICS OF SOUND OR NOISE.}

The various physical characteristics of noise or vibration, the way they travel through materials, and how they react with the surrounding environment can be measured. In many cases they can be predicted, analyzed and explained.

When acousticians or noise control engineers get together in technical discussions about noise, words like sound power, sound pressure level, intensity, 
frequency and decibels frequently are mentioned. While these terms are by no means household words, most people have a rough understanding of what they represent. We perceive frequency as pitch, intensity as loudness, and are likely to be concerned with the general level of the noise rather then the absolute over-all sound power level.

We know that decibels (commonly abbreviated as "dB") have some relation with the loudness and powerfulness of sounds. Someone living near an airport complains that the aircraft flying over his house produced a sound pressure level greater than $100 \mathrm{~dB}$ in his backyard. We see from the newspapers that there is a debate as to whether heavy industrial noise should be limited to 85 or $90 \mathrm{~dB}$ for any employe's exposure during an eight-hour working day. On the other hand, we hear remarks that a room meeting the $20 \mathrm{~dB}$ noise contour represents very quiet conditions; that an ordinary office has a noise level of 40 to $50 \mathrm{~dB}$; and someone will mention that at an ordinary symphony concert the sound levels in the audience range from some 30 or $40 \mathrm{~dB}$ up to $95 \mathrm{~dB}$.

\section{Decibel, usually written $\mathrm{dB}$}

The decibel was originally used in the design of telephone and telegraph lines. It is more common than the unit from which it was derived, for it is one-tenth of a "Bel", a unit named for Alexander Graham Bell by experts in telephony. (You can recognize this as a fate that also befell the meter as a unit of length; small lengths are more likely to be described in centimeters, large distances in kilometers.) Originally, the ratio between the power arriving at the end of a long transmission line and the power put into the line at its input was expressed as a loss in decibels; the intensification of a signal passing through an amplifier can be described by giving the ratio between the power of the output signal for a given input signal as a gain in decibels. The decibel scale counts ratios in powers of 10 . A power amplifier with a gain of $10 \mathrm{~dB}$ has multiplied the input power by a factor of 10. An amplifier with a gain of $40 \mathrm{~dB}$ has multiplied the power at the input by a factor of 10,000 (10 × $10 \times 10 \times 10)$.

The decibel scale offers a great advantage in acoustics because of the very large range of power that characterizes the sounds we are able to hear. Consider for a moment the range of sensitivity of the human ear. If we were to assign the value of one unit to the smallest flux of sound power that the ear can hear, commonly referred to as the "threshold of hearing", then a value of one million million units would be given to the greatest power that the ear can withstand without suffering immediate discomfort, which defines the "threshold of pain". (The ratio of one million million is written by engineers as $10^{12}$, meaning that twelve successive multiplications by ten of the threshold power would be needed to produce this threshold of discomfort.) If one were to plot this enormous range on graph paper with graduations of $1 / 25$ inch (1 $\mathrm{mm})$ per unit of sound power, it would leave the threshold of discomfort at the far end of a sheet of paper 62 miles $(100 \mathrm{~km}) 1$ ong. Sorted out by powers of ten on the decibel scale, the range of sound power level between the threshold of hearing and the threshold of pain can be compressed between the limits of $0 \mathrm{~dB}$ and $120 \mathrm{~dB}$ respectively, and by choosing $1 \mathrm{~mm}$ per $\mathrm{dB}$, can be plotted easily on a graph the size of ordinary writing paper.

On the decibel scale, the sound power level of $0 \mathrm{~dB}$ does not indicate an absence of sound; it indicates a level of sound, at a frequency of $1000 \mathrm{~Hz}$, (one Hertz = one cycle per second, see the discussion of Frequency and Pitch) just barely audible to a person with normal, unimpaired hearing. It turns out that a $1-d B$ difference in sound level is about the smallest relative change that the average person can detect.

\section{Sound Level Meter:}

Sound levels are measured with a sound-level meter, basically an electronic instrument calibrated to read sound level directly in decibels. It has a microphone that converts a sound-pressure variation in the air into an electrical signal, an amplifier powered by a battery to raise the signal level enough to operate an indicator needle, and an attenuator to adjust the signal level within the range of the meter's scale.

For its basic setting, the sound level meter reads the total of all sound within the range of frequencies heard by the normal human ear. As an example of what the meter indications signify, we present in Table 1-1 the sorts of readings you would see if you were to take a sound-level meter in your hand and expose its microphone to common sources of sound in your environment.

Now, if your ear behaved just like the simple sound level meter, the direct 
TABLE 1-1

COMMON NOISE SOURCES AND LEVELS

Noise Source

Near large jet plane at takeoff

Air-raid siren

Threshold of pain

Sound Level Meter Reading, $\mathrm{dB}$

Thunder, sonic boom

Garbage truck, trailer

truck, at roadside

Power lawn mower, $5 \mathrm{ft}$

$(1.5 \mathrm{~m})$

Alarm clock, vacuum cleaner, garbage disposal

Freeway traffic, $50 \mathrm{ft}(15 \mathrm{~m})$

Conversational speech

Average residence

Bedroom

Soft whisper, $15 \mathrm{ft}(4.5 \mathrm{~m})$ away

Rustle of leaves

Breathing

Threshold of hearing
140

130

120

100

0
Ratio, Sound Power to Hearing Threshold
Sound Pressure at Meter Microphone, Newtons $/ \mathrm{m}^{2} *$
$10^{14}$

$10^{13}$

$10^{12}$

20

$10^{11}$

$10^{10}$

2

$10^{9}$

$10^{8}$

0.2

$10^{7}$

$10^{6}$

0.02

$10^{5}$

$10^{4}$

0.002

$10^{3}$

$10^{2}$

0.0002

$10^{1}$

$$
1\left(=10^{0}\right) * *
$$

0.00002

(In the modern metric units, sound pressures are given in "microPasca1s"; 0.00002 newtons $/ \mathrm{m}^{2}=20 \mathrm{microPascals.)}$

*The power in a sound wave is proportional to the square of the sound pressure.

$* *$ The threshold of hearing is about $10^{-16}$ watt $/ \mathrm{cm}^{2}$; at all times you are 1 istening to a very small fraction of a watt.

readings of the meter would be all that you would need to measure the extent of a noise problem. However, high-pitched sounds -the calls of birds, the upper notes of a piano, insect chirps, the consonant sounds of speech, whispers -- all can attract your attention at low sound levels, in fact at levels so low that sounds of low frequency and similar intensity, as for example a distant airplane, might easily go unnoticed. The ear is particularly sensitive to sounds in the middle of the audible range, but has less sensitivity at the extremes of the range. In a sound level meter, this property is approximated by equipping the meter with filters that screen the sounds so that the sensitivity to sounds of different frequencies resembles that of the ear; these filters are called weighting networks.

\section{A-Weighting:}

We can give you a picture of the way in which the ear weights sounds of equal level but different frequencies by showing you a set of "equal loudness level contours". These values are measured by asking subjects to match the loudness of a test sound against a standard sound. Usually the standard sound is a pure tone whose frequency is $1000 \mathrm{~Hz}$ (about the top note of a soprano's range). A set of these equal-1oudness contours is shown in Fig. 1-1, and we have superimposed some typical 


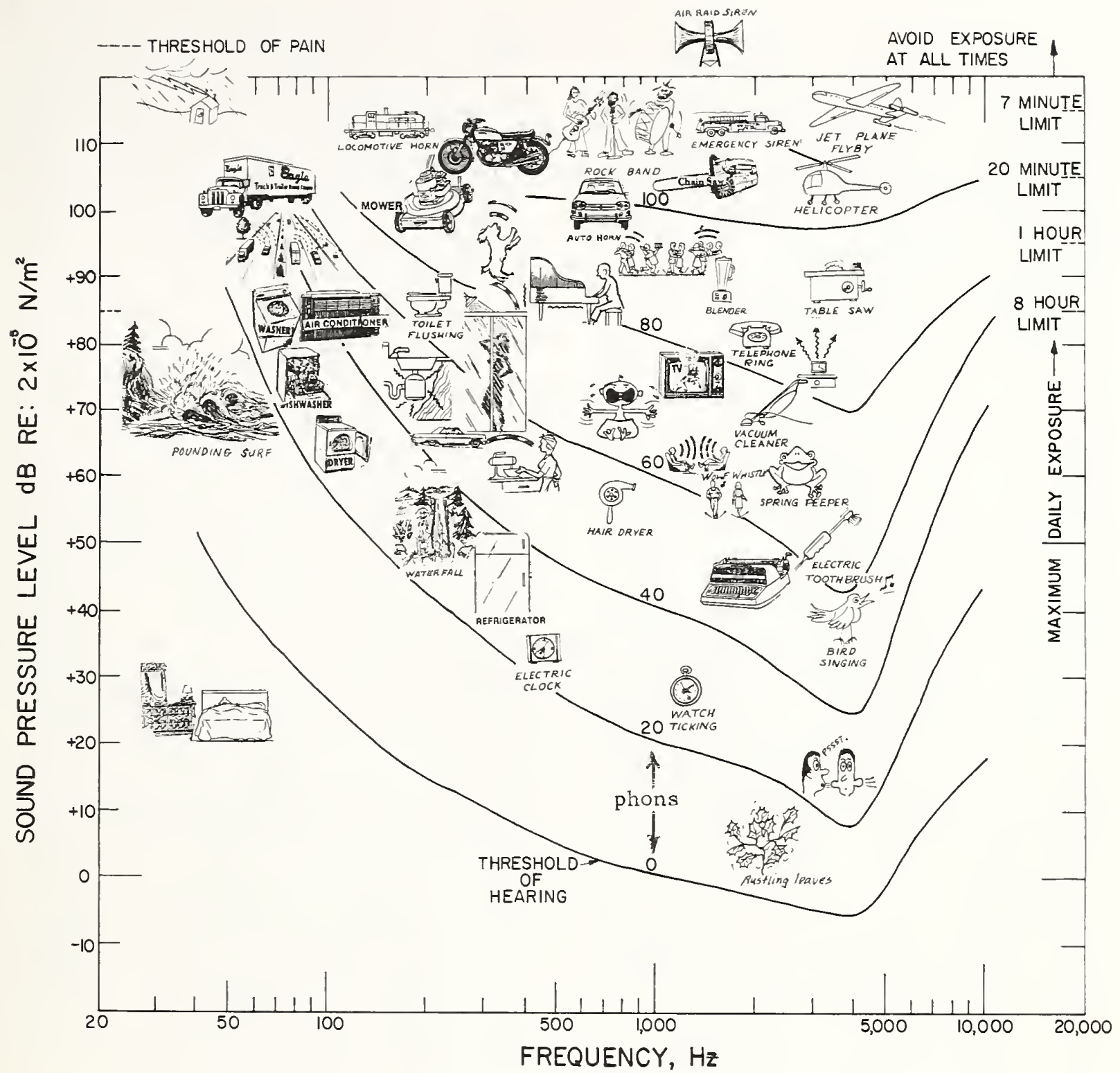

Fig. 1-1. COMMON SOUND SOURCES PLOTTED AT THEIR DOMINANT FREQUENCIES AND LEVELS AS TYPICALLY HEARD BY THE OBSERVER

sound sources at their appropriate sound levels and at the dominant frequency that determines their relative loudness. Until fairly high levels of sounds are reached, the contours are more or less parallel to one another.

The A-weighting network on a sound level meter is designed to adjust the sensitivity of the meter to sounds of different dominant frequencies so that its indications show more nearly how the ear might respond to moderate sound levels. The
A-weighting of a sound level meter has been found to agree reasonably well with subjective response to the loudness of sounds. When an A-weighting network has been used to filter the signals applied to the output meter of the sound level meter, this fact is indicated by writing "dB(A)". As an example of what the filter does, consider an air conditioner whose noise you set out to measure. Much of the fan and air-turbulence noise is low-frequency; some hum at low multiples of the power-line frequency is radiated by the fan motor. The 
whistling of air through the grille is a high frequency sound. In the absence of any weighting, the sound level meter might indicate $70 \mathrm{~dB}$ as the sound level at the grille. With the A-weighting network, some of the low-frequency power is attenuated in the meter weighting circuit, and with the A-weighting network in place, you might get a reading of only $58 \mathrm{~dB}$. It would be customary to report this reading as "58 $\mathrm{dB}(\mathrm{A})$ ".

At high sound levels ( $90 \mathrm{~dB}$ and above) the ear seems to perceive sounds more nearly like a "flat" or unweighted sound-level meter. Sometimes this is designated the " $\mathrm{C}$ " weighting. There is also a " $B$ " weighting that is used to approximate the loudness weighting ascribed to the ear for a 70-dB contour -- it is intermediate between $A$ and $\mathrm{C}$ weightings.

\section{The Notation "dB(A)":}

This guide will follow the practice of reporting sound level meter indications read with an A-weighting network as $\mathrm{dB}(\mathrm{A})$. Many regulatory agencies of state and local government use only the A-weighted indications when they are dealing with human responses. When in this guide sound-level meter readings are indicated merely as in $\mathrm{dB}$, they represent the unweighted readings of the sound level meter, obtained with the "flat" setting of the meter.

\section{Frequency and Pitch}

The number of complete to-and-fro vibrations that a source of sound makes in one second is called the frequency of vibration. For example, if a loudspeaker cone moves back and forth 100 times in a second, the frequency of the tone it radiates is said to be 100 cycles per second, (the cone has executed 100 cycles of the same motion within one second) or, in modern metric terminology, 100 Hertz (abbreviated $\mathrm{Hz}$ ).

The ear senses the frequency of a sound as the pitch. Sounds with frequencies near $30 \mathrm{~Hz}$ are sensed as having extremely low pitch, whereas sounds near the upper frequency limit of hearing at $15,000 \mathrm{~Hz}$ are judged to have an extremely high pitch. The noise of bacon sizzling in a pan is judged to be high-pitched, whereas the rumble of distant thunder is low-pitched. The small, high-speed wings of a mosquito radiate a higher-pitched whine than do the larger, somewhat more slowly vibrating wings of a bumblebee.
The ear recognizes the doubling of frequency as producing a similarity of tone quality. Musicians call this change the octave. To produce the same pitch change in different parts of the frequency range, the interval must be adjusted to be proportional to the frequency. The scale of frequencies in Figure $1-1$ is adjusted for this property of hearing, and you may notice that each doubling of frequency takes up the same horizontal interval in the figure.

Loudness, Sound Pressure and Intensity

Loudness may be described as a human or subjective measure of the pressure or intensity of sound. It can be ordered on a scale with limits ranging from whisper-soft to painfully loud. We have already seen that the loudness of a sound of a given intensity depends upon its frequency or pitch. However, if we present a sound of a given frequency at different levels and ask a person to assign a number representing its loudness, we find that - as a "rule of thumb" - doubling of loudness corresponds roughly to a $10 \mathrm{~dB}$ increase in sound level. (Experiments give from 10 to $12 \mathrm{~dB}$ ). Table 1-2 relates changes in sound level to the average person's perception of loudness.

TABLE 1-2

SUBJECTIVE RESPONSE TO CHANGES IN SOUND LEVEL
Change in Sound Level

$$
\begin{aligned}
& \pm 1 \mathrm{~dB} \\
& \pm 3 \mathrm{~dB} \\
& \pm 5 \mathrm{~dB} \\
& \pm 10 \mathrm{~dB} \\
& \pm 20 \mathrm{~dB}
\end{aligned}
$$

\section{Change in Loudness}

Requires close attention to notice

\author{
Barely perceptible \\ Quite noticeable \\ Dramatic; nearly twice or \\ half as loud \\ Striking, fourfold change
}

You can see now that Figure 1-1 gives you a picture of how the ear weights sounds of equal level but different frequencies. The ordinary noise sources are shown at their typical sound-level meter readings in $\mathrm{dB}$, plotted at their dominant frequencies. These are the indications that the sound-1evel meter would yield as measured at the position where the sound would be most likely to be experienced: For some appliances, such as a blender or a power mower, at the position of the operator; for vehicles on a highway, at curbside; for 
aircraft overflights and neighborhood noise sources, outside the home; for such sounds as the ocean, bird calls, or thunder, at the position of the observer out-of-doors. Superimposed on this plot are contours of equal loudness for pure-tone sounds, depicted by the solid curved lines. On a given contour any pure tone at the sound level and frequency indicated will be judged by the average person to be equally as loud as any other tone along the curve. For example, on the lowest contour, the "threshold of hearing", a 50-Hz tone at a level of about $45 \mathrm{~dB}$ sounds equally as loud as a $500-\mathrm{Hz}$ tone at $5 \mathrm{~dB}$. The audiofrequency spectrum from 1000 to $8000 \mathrm{~Hz}$ (approximately the two top octaves of the piano and the next octave beyond) covers the region where your ear is most sensitive. Some of the more distressing sounds, such as the squeaking of chalk on a blackboard lie in this region. It is in this region, also, that the ear is most vulnerable to damage by noise or disease.

Phons:

In Fig. 1-1, the spacing of the contours is chosen to be in even increments of the $1000-\mathrm{Hz}$ standard tone, as read on a sound-level meter. The contours connect sound level values where the loudness is alike. Each contour is rated numerically for the sound-level of the 1000-Hz tone with which other tones on the contour are comparable. Although they, too, are written as $d B$, the units related to the contours are no longer sound level, but "phons", and now represent a level of loudness perceived to be equal in effect to the $1000-\mathrm{Hz}$ tone through which the contour passes at a sound-level indicated in the decibel scale on the left-hand margin of the graph.

\section{Pure Tone and Broadband Noise}

Throughout this guide some noises may be described as pure tones, or as having pure-tone components, whereas other noises may be described as being broadband in character.
Pure Tone:

A pure tone is the sound radiated by a source vibrating at a single discrete frequency. For example, the sound emitted by a tuning fork that is properly struck is remarkably pure in tone. Similarly, striking a single key on a piano or plucking a single guitar string produces a relatively pure tone. The average person can produce a reasonably pure tone simply by puckering his lips and whistling a note. A chord struck on the strings of a guitar is an example of a tone made up of a number of pure-tone components.

Broadband Noise:

Broadband noise, on the other hand, is a complex mixture of sounds of different frequencies. Often these mixtures are changing rapidly with time. The sound produced by a nearby waterfall or a pounding surf is relatively broadband in character.

In many instances, noise sources radiate both broadband noise and pure-tone components. A jet plane at take-off, for example, radiates a combination of such noises. There is the shrill whine of the turbine, which may have a pure-tone, whistle-like character, superimposed on the broadband roar of exhaust noise. The vacuum cleaner emits a siren-like sound from its impeller, generates whistling noise in some corrugated hoses, and also produces broadband air- suction sound. Wind blowing past overhead power lines often generates a pure- tone whistling sound which stands out against the background of the broadband noise of the wind.

There is some evidence that pure tones are judged to "grow" in loudness as they endure over a time interval of a number of seconds, whereas broadband noises soon reach a plateau of loudness. This is not indicated by the usual methods of measuring equal-loudness contours because comparisons ordinarily are made between sounds of similar durations. However, this feature does have a bearing on the choice of procedures for noise abatement. Special effort to remove pure tones is advisable. 
When you are in a sound field whose level as read on a sound-1evel meter is 70 $\mathrm{dB}$ or more, even though you know consciously that you are in no hazard, some part of your body tries to run away. The signs of this are the common symptoms of nervous stress: Your heart beats more quickly; breathing becomes shallower and faster; the pupils of your eyes dilate; the small blood-vessels in your skin contract; your blood pressure rises. These are the actions of a body arousing itself to escape. During sleep the sound level need only exceed $55 \mathrm{~dB}$ to produce changes resembling the waking stress for $70 \mathrm{~dB}$.

You can become "accustomed" to the noises in your environment, but a11 that this means is that you can become accustomed to eliminating the noise from your conscious attention. The physiological effects persist. If you are compelled to work in noisy surroundings, the only warnings of acoustically-induced stress may be signs of fatigue and nervous strain.

Experiments with anesthetized animals show that the stress reactions persist at the same levels whether or not the animal is aware of them. Adaptation to the stress does not occur. Studies of noise reactions of people living in crowded neighborhoods also have shown that, over a period of years, the rate and kinds of complaints about noise did not change.

No one knows how many of the stress-induced diseases of our modern era are made more prevalent by the rising noise levels in our densely-populated cities. Note that these levels do not affect hearing. Nor do the results mean that all noise should be excluded. Studies of the effects of noise upon work indicate that some degree of ambient sound (which might as well be pleasant, such as the sounds of a fountain, or background music) helps maintain arousal during repetitious tasks; only the very highly-demanding mental tasks are done better in wholly quiet surroundings.

Noise damage to hearing is an insidious process. The immediate effects do not always indicate the ultimate results of exposure. Exposure to a mildly excessive noise level results in a temporary desensitization of the ear, the so-called "temporary threshold shift", a temporary loss of the ability to detect faint sounds. Noises likely to cause a temporary threshold shift are encountered during subway rides and airplane trips. Some degree of temporary threshold shift may be detectable even on the day following exposure, but ultimate recovery can be complete.

However, this pattern of threshold rise and recovery sometimes goes on through a number of cycles without any further evidence of damage, and then there may be a sudden failure to make complete recovery. This permanent effect - "noise-induced permanent threshold shift", sometimes abbreviated in capitals as NIPTS - is a sensorineural hearing loss. That is, the damage is located at the auditory nerve, itself, with serious consequences.

Studies of NIPTS over a period of time have shown some evidence that persons are particularly likely to suffer hearing damage when they are going through physiological change or enduring physical stress such as rapid growth or illness. Thus, the common practice among teenagers of listening to extremely loud amplified music for long periods of time, especially through earphones, may have very serious consequences. Young persons, in particular, frequently engage in several activities each of which may be sufficiently noisy to constitute a hearing hazard. For example, it is not unusual for a high school student to earn spending-money during summer vacation by mowing neighborhood lawns with a typical, usually very noisy, power mower. The same person also may rehearse several hours a week with a loud rock and roll band, then play for hours at the saturday night dance. And finally for excitement or recreation, the student may spend his leisure time riding a powerful but noisy motorcycle, water skiing behind a noisy high-speed motorboat, or perhaps skeet shooting at the gunnery range. Although no one of these activities may cause any hearing impairment for short or infrequent exposures, they cannot be taken a11 together at frequent intervals without risking hearing damage.

\section{PRECAUTIONS}

WARNING 非1: Because we have no reliable way to predict the degree of permanent loss from temporary threshold shift, a conservative policy is to avoid situations 
giving rise to temporary threshold shift. Any person whose hearing does not recover within a few days after an exposure that induced a threshold shift should avoid any further exposure to sounds of that level, and should take positive action to protect his hearing. He should not return to that environment without protective equipment.

After exposine to sound levels of $85 \mathrm{~dB}$ and above for lengthy periods of time, a significant number of persons will be found to have a permanent hearing loss (NIPTS); this degree of exposure essentially doubles the incidence of hearing loss beyond what would occur from mischance and disease in the course of aging. For exposures above this level, the incidence of losses rises fairly rapidly with the sound level to which persons are exposed. Since there is some reason to believe that $80 \mathrm{~dB}$ represents the point from which the damage risk begins to be significant, it is obviously a good idea to avoid continuous exposure to any levels above $80 \mathrm{~dB}$ for any length of time comparable to a working day, and to wear ear protection if exposure is necessary. The potential damage point is indicated fairly reliably by conversational effort.

WARNING 非: If you have to raise your voice to carry on a conversation at a distance of 3-5 feet from the person with whom you are talking, you may be subjected to a potentially damaging exposure. (See Fig. 7-1, Chapter 7) Not only does it inconvenience you in your conversation at the moment; it may make it difficult for you in the future.

Figure 2-1 is a diagram of the process whereby the sounds arriving at your ear through the air reach the auditory nerve at the entrance to your brain. Like any well-engineered system, your ear has a few external protective devices. If you are exposed to an excessively loud noise, the muscle tensing your eardrum pulls taut,

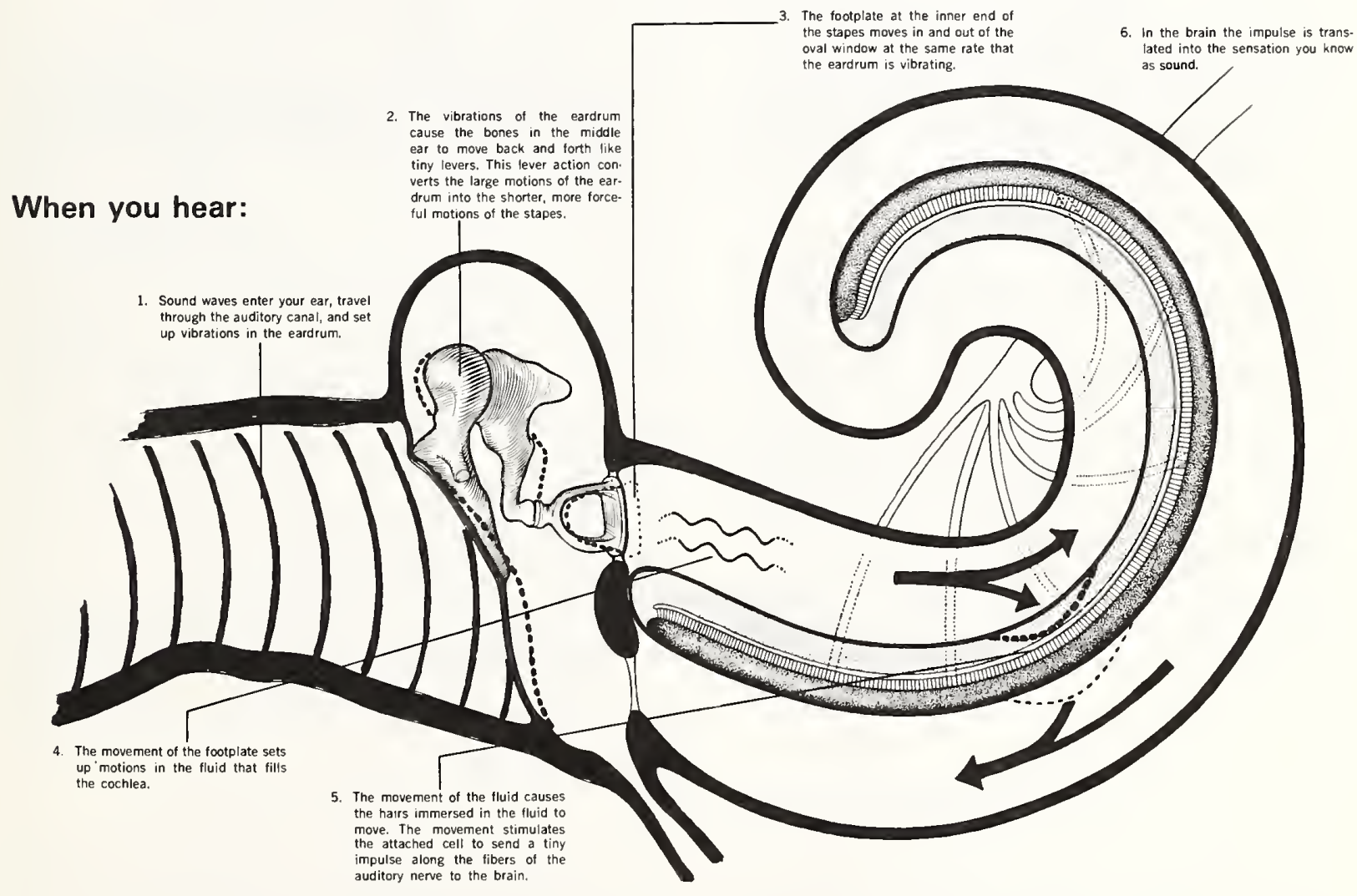

Fig. 2-1. A SCHEMATIC DIAGRAM OF HOW THE HUMAN EAR FUNCTIONS 
desensitizir ${ }$ Ll. This "stapedius reflex" takes place at sound levels between 80 and $95 \mathrm{~dB}$. The point of onset of the reflex varies with individual sensitivity. Some persons can operate the stapedius reflex at will; from their experience it is evident that the desensitization is of the order of $20 \mathrm{~dB}$. Thus the protection afforded by the stapedius reflex is important.

However, a burst of intense sound pressure too abrupt to give the stapedius reflex time to act can occur. This is particularly true of a shock-wave from a gun-shot or a pressure burst from a box on the ear. In that event, the drum membrane is likely to rupture, causing a temporary loss of hearing and a hazard of middle-ear infection. However, if no infection follows the injury, the membrane will heal. Except for the effect of the weight of the heavier scar tissue that is likely to form, the ultimate sensitivity of the ear will have suffered little change. Desensitization due to scar tissue may be enough, in some individuals, to lower the response of the ear to high-frequency sounds, where the critical consonant sounds, of speech are located, and where the cues to musical tone quality are transmitted. (Since it is sometimes necessary to lance the drum membrane in cases of middle-ear infection, with a consequent formation of scar tissue, it is clear that any ear infections must be attended to at once to avoid a residue of permanent loss.)

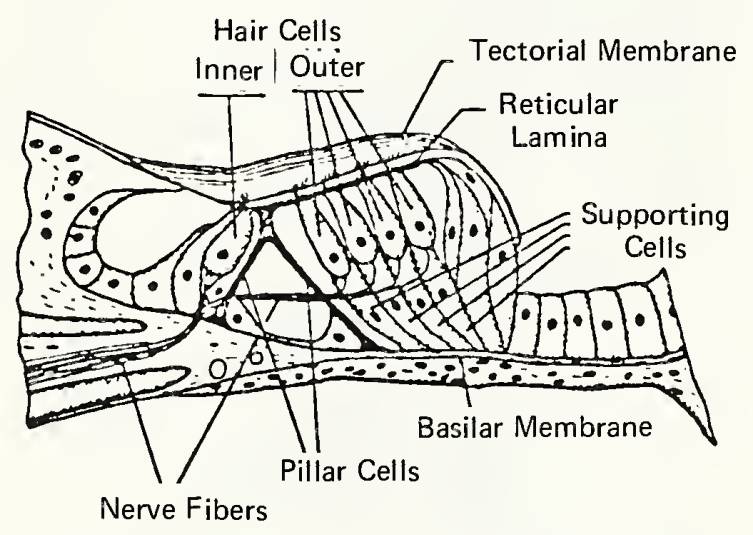

(A) NORMAL ORGAN OF CORTI

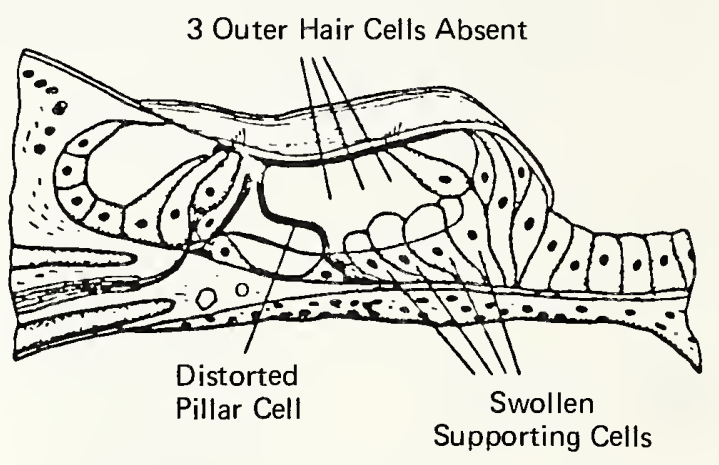

(B) PARTIAL INJURY

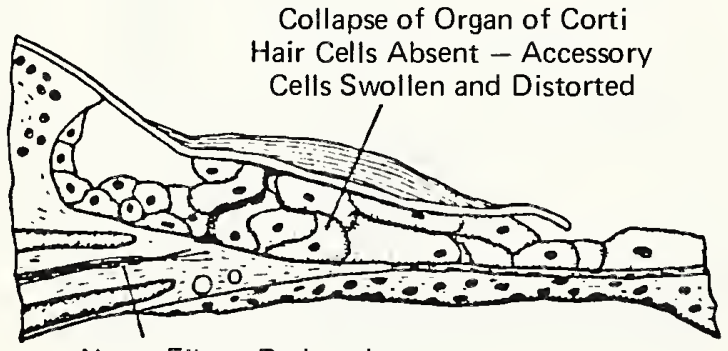

Nerve Fibers Reduced in Number

(C) SEVERE INJURY

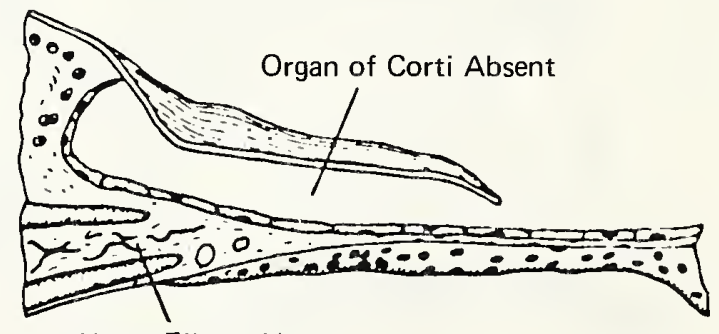

Nerve Fibers Absent

(D) TOTAL DEGENERATION

Fig. 2-2. DRAWINGS OF THE HUMAN ORGAN OF CORTI ARE SHOWN THAT ILLUSTRATE THE NORMAL STATE, PANEL A, AND THE INCREASING DEGREES OF NOISEINDUCED PERMANENT INJURY, PANELS B, C, AND D. THIS IS WHAT YOU WOULD SEE IN A SECTION VIEW CUT ACROSS THE REGION OF THE HAIR CELLS SHOWN IN Fig. 2-1, INNER EAR. 
It is damage to the hair cells in the inner ear that causes the irretrievable losses that are associated with noise damage. From experiments on animals, it has been found that loss of $15-20 \%$ of the hair cells that respond to high frequencies will produce a rise in threshold of about $40 \mathrm{~dB}$. This loss, for human beings, occurs first in the region most important for discrimination of the consonant sounds of speech - in the range from 4000 to $6000 \mathrm{~Hz}$. If you look at the diagram in Fig. 2-1, you can see that losing a fraction of the hair cells is comparable in some ways to the loss in vision that would occur if scattered areas of your retina were destroyed. Although the effect observed by measuring your threshold of hearing is simply that your threshold has risen, which is the same sort of observation that would be made if the injury were in the drum or in the chain of bones in the middle ear, something much more serious has been lost: speech becomes difficult to understand.

Loss of hair cells impairs the resolution that your ear can bring to bear on sounds. Even after amplification, the loss of resolution remains. As you can see from Fig. 2-2, which shows the damage to hair cells as a result of noise exposure, nerve endings have been put out of action. The loss impairs particularly the ability to understand conversation in the presence of noise. With a severe loss of hair cells, although a person can use a hearing aid to permit him to hear sounds at levels comparable to those heard by persons with normal hearing, he may not be very successful at recognizing speech. The average person who has suffered a sensorineural hearing loss that raises his unaided threshold above the normal by $60 \mathrm{~dB}$ will generally have a significant degree of difficulty in understanding speech even while he wears his hearing aid. Thus a permanent threshold shift produces a permanent impairment of hearing, and the only practical remedy is prevention. Figure 2-3 shows recovery times in quiet surroundings recommended to minimize hearing loss caused by exposure to hazardous sound levels.

If you have been exposed to an excessively intense noise, you are likely to notice a ringing in your ears as an after-effect. This is called "tinnitus", and it seems to represent a spontaneous firing of signal impulses by the hair cells. Also at sound levels that are potentially damaging to hearing, you may notice that music acquires a complex, sometimes

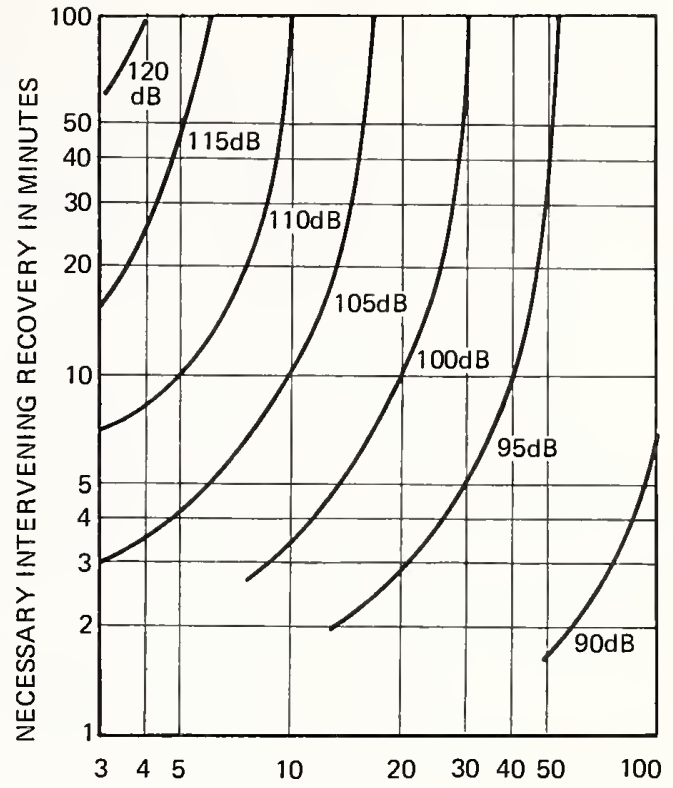

NOISE BURST DURATION IN MINUTES

PARAMETER: SPL 600 TO $1200 \mathrm{HZ}$ OCTAVE BAND

Fig. 2-3. RECOVERY TIMES AND MAXIMUM EXPOSURE
ALLOWED TO REMAIN BELOW PROPOSED DAMAGE-RISK LIMITS (WARD, 1966)

discordant sound. This is because the ear produces mixtures of tones instead of merely transmitting the sound stimuli. Only the rhythmic patterns of music may remain esthetically pleasing. At these levels, some effect is sensed on the semi-circular canals of the ear that normally respond to orientation and balance. This gives a sensation of dizziness, which is perhaps cultivated by devotees of rock-music as a pleasurable sense of detachment, analogous to drunkeness. Like the over-consumption of alcohol, continuous very loud sounds lead to an end-point of seasickness and nausea. You can literally experience an "acoustic hangover".

WARNING 非3: At still higher sound levels, a "tickle" sensation is felt in the ear, and with another $10 \mathrm{~dB}$ rise of level, a sensation of pain intrudes. The pain is an important though perhaps tardy warning. Very little exposure at the threshold of pain is needed to produce a permanent hearing loss in susceptible individuals. Another serious flaw in this warning system is the fact that at present we cannot predict who is susceptible. (There is some reason to suspect that susceptibility is 
enhanced by large-scale changes in the physiology of the body, as in adolescence.)

It is a fact that persons in noisy occupations and with noisy avocations are found generally to have various degrees of permanent, sensorineural hearing loss. Among the pleasures having more devastating consequences are rock music, target shooting and snowmobiling. Hearing loss due to gunshot in warfare is very prevalent.

The readings of an ordinary sound-level meter will fail to indicate the true level of rapidly peaking sounds because the needle of the meter is too sluggish to follow the sharp pressure changes that occur on impulsive sounds like shots, or even the sounds of a poorly-muffled internal combustion engine, which after a11, derives its power from the explosions confined in combustion chambers. This makes it difficult to estimate exposure to impulsive noises.

\section{HEARING PROTECTION}

We are hampered in preventing a great deal of hearing loss because of a degree of bravado that causes some people to shun wearing hearing protection. Since hearing loss is subtle and invisible, perhaps it seems illogical to choose a visible means of protection. Some types of ear protectors are shown in Fig. 2-4.

Simple insert-type ear protectors are usable where perhaps 10-20 dB of protection is sufficient to eliminate the chance of hearing loss. In general, these are more effective for high-frequency sounds such as whistles than for low-frequency sounds such as machinery noise or jet turbulence. Cotton worn in the ears is not very effective, only a little better than nothing. However, in a pinch, you can derive some real help from cotton soaked in vaseline or soft wax (to increase its mass and sealing power), and pressed gently into your ears. Commercial ear protectors of the deformable soft plastic type provide still better protection. At most, you can count on internally-worn ear protectors for a 10-20 $\mathrm{dB}$ reduction.

Really effective protection is offered by over-the-ear muffs, which look like a relatively large pair of headphones. You may have seen these on the personnel working at airports. For maximum protection, both muffs and inserts may be worn. Even the combination of muffs and ear protectors, however, can offer little more than $40 \mathrm{~dB}$ protection, for sound can enter the body by other paths, entering via the chest cavity, the throat, and the bones of the head. If you translate that information into the conditions of your environment, you cannot remain for any length of time around sound levels in excess of $120 \mathrm{~dB}$ without walling yourself off from the source of sounds. In operating noisy machinery, workmen may find it necessary to observe and control the operation from the window of an isolation booth.

If you are concerned about appearances, be assured that these are not as obvious as hearing loss, for a hearing loss at high frequencies destroys the referee that enables you to monitor your own speaking. Not only do you have trouble hearing other people, because your hearing loss slurs and obliterates the consonant sounds of speech, your own speech becomes like what you hear.

How can you protect your hearing? The following notes may help:

Avoid Situations Where

1. You have to raise your voice to converse with anyone.

2. Ordinary melodic music sounds discordant.

3. You can't manage to talk over the telephone.

4. Sharp noises are repeatedly making your ears ring.

5. Everything seems too bright and too loud.

6. The racket makes it difficult to "think straight".

7. You begin to feel detached and a little dizzy.

8. In a short while you begin to feel tired and dazed.

9. The noise makes you feel seasick.

Your Hearing May Have Begun To Deteriorate When It Seems That

1. You have trouble recognizing what is said from the stage or pulpit.

2. You have to ask people to repeat what they say.

3. People ask you to repeat what you say.

4. You lose the thread of conversation at the dinner table.

5. The birds seem to have stopped singing.

6. You miss the telephone bell or the doorbell. 


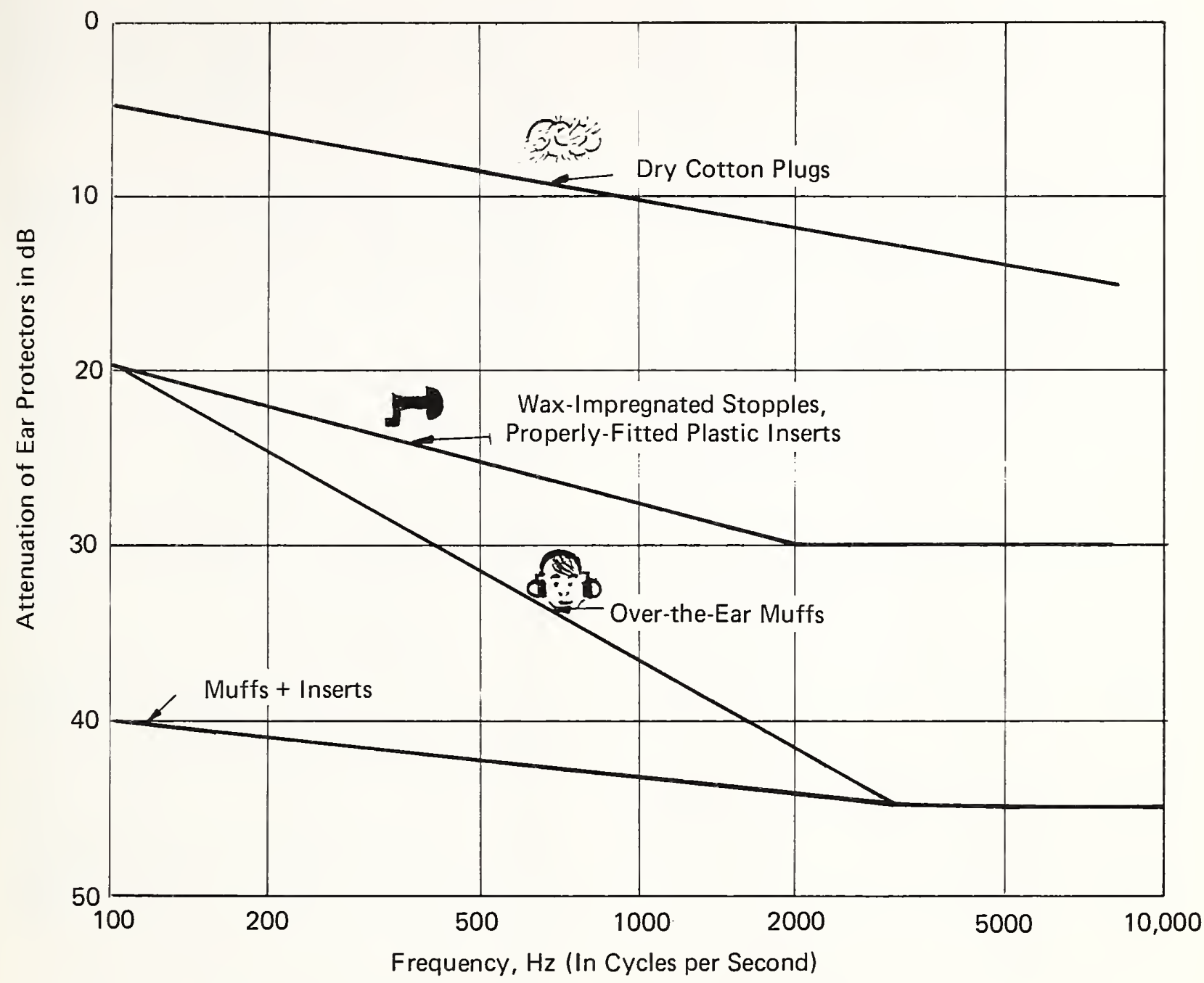

Fig. 2.4. SOUND ATTENUATION CHARACTERISTICS OF VARIOUS TYPES OF EAR PROTECTORS

7. Your family repeatedly asks you to turn down the TV or radio.

8. People seem to have begun to mumble.

Be Cautious in the Use of These Devices

If you garden:

Power lawnmowers, chain saws, mulchers, lawn vacuums, lawn edgers.

For the sportsman:

Guns, speedboats, snowmobiles, motorcycles, all-terrain vehicles.
Even if you are the sheltered, indoor type of person:

Power

tools,

"industrial vacuum-cleaners", superpowered Hi-Fi, rockmusic bands.

HOW TO AVOID RISKING YOUR LIFE AT NOISE LEVELS NOT DAMAGING TO HEARING

A high sound level can conceal the presence of rival sounds in your environment, or reduce their apparent loudness, thus removing their capacity for attracting your attention. This concealment, known as masking, can prove fatal. 
For example, in a modern car with stereo and air-conditioning running, someone we know drove happily out of her driveway and collided with a fire engine. The car was a total wreck. Two factors militated against her: In the first place, the closed car windows attenuated exterior sounds. In the second place, although the car radio masked the motor noises and the air conditioner with pleasing sounds, it also masked out the warning siren and the engine noise of the fire truck. Since the car noises were in themselves not negligible, she was of course actually operating the radio at unduly high sound levels. In order to have overridden all this sound and caught her attention, the fire engine's siren would have had to be intense enough to offer a risk of hearing damage to the firemen themselves and to pedestrians on the street.

The most common function of a siren is to alert motorists and pedestrians to clear the road for an approaching emergency vehicle. Tower or roof-mounted sirens, on the other hand, alert the populace to some impending danger, such as an air raid, or summon volunteer fire fighters as the case may be. Although the noise is intense enough to, and often needlessly does, awaken large segments of a sleeping community, it fails all too frequently to achieve its main objective, that is, to alert the motorist. This failure is due to one or a combination of the following factors:

-vehicle windows are closed

-high noise level in the vehicle due to operation of radio, fan or air

conditioning equipment or the

intrusion of engine or traffic noise

-preoccupation or distraction of the driver.

For the motorist, the ideal warning device would be a small buzzer or alarm with perhaps an intense flashing light installed on the dashboard of his vehicle that could be triggered or set off by a short range radio frequency transmitter on the emergency vehicle. Both the warning buzzer and light could be modulated to buzz and flash at a gradually increasing rate as the emergency vehicle approached, to alert the motorist as to its proximity.

Similar warning systems could be installed in the station house and homes of volunteer firemen. If the installation of such equipment were made mandatory in a11 emergency and automotive vehicles, communities would benefit from a dramatic curtailment in the use of a highly disturbing source of noise pollution and perhaps a significant improvement in the safety of conducting emergency or rescue missions.

Lesson 1: In order to hear sirens of emergency vehicles while you are driving an air-conditioned or heated' car in city traffic, do not close it tightly. Leave an opening of perhaps half an inch (about $1 \mathrm{~cm}$ ) in the window next to the driver's seat.

Leaving the window next to your ear raised enough to prevent "line-of-sight" access from the sounds of the road has some advantage. It will generally not interfere with the sounds of sirens and warning whistles, which are ordinarily pitched in the range from 500 to $3000 \mathrm{~Hz}$, but some of the high-frequency road noises generated by tire treads and exhaust turbulence will be kept from reaching your ear at levels high enough to induce acoustic fatigue. Use your radio sparingly and at low volume when driving in any degree of traffic. You have more at stake than your hearing. However, you must still be alert because the partially open window offers no guarantee that you will hear an approaching siren.

A careful industrial study has shown that persons in noisy environments gradually require longer times to react to emergencies; we say their reaction times have increased. This points to a special hazard: Reaction time is known to have an influence on accident rate. (One of the important consequences of the consumption of alcohol, and of such medicinal agents as tranquilizers and antihistamines, is an increase of reaction time.) Further, in the noise exposure cases it was found that although for the first day or two of exposure, a rest period of an hour or more restores the reaction time, continued exposure to noise in succeeding days is no longer compensated entirely by rest.

In the industrial experiment, persons working for an eight-hour day were tested before and after each four-hour work session, with a lunch hour between. Each group in this study had just returned from vacation. By Wednesday of each week, the reaction time in the morning was lengthened a little, and full recovery did not follow the noon hour. By Thursday, and even further on Friday, the reaction time was lengthened at al1 times, and recovery was poorer. On Friday afternoon, the reaction time was nearly $50 \%$ longer than the normal 
level following extended rest. This was not due to the work: Half of the subjects of the study were merely sitting in the noisy environment beside their working companions.

Lesson 2: For maximum safety, you should break up working spells in a noisy environment by taking a full day's rest in a quiet area at frequent intervals. It is worth noting that you can enhance the safety of your family on long trips by automobile simply by breaking the trip after four hours' driving, for a rest of at least an hour in a quiet surrounding, and by making a one day stopover for every three days, preferably two days of driving. The definition of "quiet" has been found by experiment, as well: Full recovery from acoustic exposure demands a quiet area in which the ambient noise is less than $55 \mathrm{~dB}$. Since the lengthening of reaction time is primarily due to the exposure to noise, changing of drivers among the travelers within the car will not avoid this problem.

Another source of accidents that can be caused by noise, which is not in itself at a physically damaging level, is the "startle reflex". The normal human reflex at a sudden loud noise is to cringe: The eyes blink and shift, the shoulders are drawn together, the head is moved downward and away from the sound, the long muscles of the arms and legs pull them toward the trunk. Probably the startling noise of a blowout is the major cause of loss of control of a car after a blowout has occurred.

Lesson 3: If you are driving a car and see a pedestrian about to cross the street, you have to measure the possibility that by blowing your horn you will trigger off a startle reflex in him and possibly in other bystanders, and weigh it against the chance that you might lack enough control to stop before you hit him. Startle reactions in bicyclists are especially dangerous because they can lose direction as well as balance. If you are riding a bicycle, you should be careful to remain in a lane clear of traffic to protect yourself against a sudden startle; for the same reason a passing motorist should allow a bicyclist a wide lane, even though the small size of the vehicle does not require it.
A reflex known as "nystagmus", a sideward back and forth scanning of the eyes, is triggered off by noise. For most persons it is unlikely to occur until sounds reach quite a high level, but for susceptible persons it may become troublesome at levels below those directly injurious to hearing. Naturally, this reaction interferes with visual acuity and the judgment of distance.

Lesson 4: Since all the protection we can suggest is only a palliative measure, it is clear that the best procedure for protection from noise is to cut off excess noise at the source. Unfortunately, to many people, noise means power. Thus, there is some feeling that there may be a virtue in noisiness. An adolescent who removes the muffler from his car feels it "sounds more powerful", thus bigger and somewhat better than before. This is no different from the reaction of the purchaser of a lawnmower or vacuum cleaner, who has a strong feeling that the sound indicates reserve power. Manufacturers of power tools report that efforts to quiet the equipment do not pay; people feel that the equipment is somehow less powerful.

Perhaps because the quieting of equipment often removes high-frequency noise preferentially, the impression is created that the action of the equipment is stifled (as a hand held over your mouth smothers your speech, letting the basic voice through but muffling the consonants). This is very far from true. Internal-combustion engines with properly resonated mufflers actually fire better, make better use of fuel, and run more steadily. Thus, the tradition among many cross-country truckdrivers of "cutting out" the muffler on open roads is actually countereffective.

To re-establish a feeling for the magnitudes involved, just remember that a sound capable of damaging your hearing need have a power flux of no more than one thousandth of a watt per square centimeter. Thus the amount of power involved in generating noise is very sma11; a mechanism using very little power can generate large amounts of sound (ask any parent). Noise is thus no criterion for the availability of usable power. 


\section{BASIC PRINCIPLES OF NOISE REDUCTION}

If you have a noise problem and want to solve it, you have to find out something about what the noise is doing, where it comes from, how it travels, and what can be done about it. A straightforward approach is to examine the problem in terms of its three basic elements: i.e., sound arises from a source, travels over a path, and affects a receiver, or listener.

\section{SOURCE-PATH-RECEIVER CONCEPT}

The source may be one or any number of mechanical devices radiating noise or vibratory energy, such as several appliances or machines in operation at a given time in a home or office.

The most obvious transmission path by which noise travels is simply a direct line-of-sight air path between the source and the listener; for example, aircraft flyover noise reaches an observer on the ground by the direct line-of-sight air path. Noise also travels along structural paths. In most cases it travels from one point to another via any one or a combination of several such paths. Noise from a washing machine operating in one apartment may be transmitted to another apartment along air passages such as through open windows, doorways, corridors or ductwork. Direct physical contact of the washing machine with the floor or walls sets these building components into vibration. This vibration is transmitted structurally throughout the building causing walls in other areas to vibrate and to radiate noise.

The receiver may be a single person, a classroom of students, or a suburban community near an airport or expressway.

Solution of a given noise problem might require alteration or modification of any or all of these three basic elements:

(1) modifying the source to reduce its noise output,

(2) altering or controlling the transmission path and the environment to reduce the noise level reaching the listener, and

(3) providing the receiver with personal protective equipment.
Let us examine the various noise control measures that can be applied in each of these categories.

\section{NOISE CONTROL AT THE SOURCE}

\section{Select Quiet Equipment}

Basically, the best way of controlling noise at its source is to select quiet equipment or appliances initially. When shopping, look for equipment that carries a low-noise certification or rating - preferably backed with a copy of the certifying laboratory's test report. Some appliances and equipment feature or advertise noise-control design or construction - look for descriptive terms such as "sound conditioned," "acoustically treated", "quiet operation", and similar phrases that may be used in advertising copy or in owner's manuals. However, you must be on guard because such descriptive phrases as "whisper quiet" may be more poetical than absolute, or perhaps an expression of hope rather than reality.

Unfortunately, the selection of mechanical equipment and appliances that feature noise ratings or that specifically call attention to noise control design is extremely limited. Thus, the buyer must resort to his own wit or know what to look for in order to choose quiet equipment. The key words to remember in making purchasing decisions are "slower" and "Iower," particularly as they apply to the operation of the equipment. The speed of moving parts, flow velocities, gas or fluid pressure differentials, and power ratings all affect noise output. By looking for equipment displaying "slower" and "lower" operation characteristics you can be assured of relatively quieter operation. For example, large slow-speed fans are substantially quieter than small high-speed fans for a given air flow; low horsepower motors are less noisy than those with high horsepower ratings; likewise low-pressure, low-velocity air ventilation or fluid distribution systems are virtually noiseless compared with their high-pressure high-velocity counterparts.

Whenever possible, the buyer should conduct a side-by-side comparison noise test for various makes or types of appliances or equipment. This can best be done by dealing 
with a supplier who carries a wide selection of appliances or equipment made by several manufacturers and who is willing to demonstrate their operation in the show room. This, of course, is possible when shopping for small domestic appliances such as vacuum cleaners, window air conditioners, etc. However, large mechanical installations, such as central heating or air conditioning systems can only be properly evaluated in-place.

The following summarizes the features that the buyer should look for and the steps he should take in selecting quiet equipment.

(1) low-noise certification,

(2) advertisement operation, evidence of noise-control design,

(3) emphasis on "lower" and "slower" operating characteristics,

(4) conduct comparative side-by-side noise tests in the dealer's showroom,

(5) request an on-site inspection of mechanical equipment of the type you are considering, that the dealer has previously installed, for the purpose of making your own evaluation of the noise output.

\section{REQUIREMENTS FOR QUIET OPERATION}

Most mechanical devices such as automated appliances and machinery are complex noise generators that contain numerous sources of noise. For example, the overall noise radiated by a clothes washer may be generated by the drive motor, gear train, pump, pulleys, cams, bearings, electrical switches, automatic valves, and water flow. Reducing the noise output of such a device usually requires a considerable amount of study to identify the major sources of noise and their respective transmission paths. In other words, it is important to know whether it is the motor, gear train or the pump that is the main cause of noise - and whether the noise is radiated directly from the source into the air as airborne noise, or whether it is transmitted structurally as vibration to other parts and surfaces of the machine which, in turn, vibrate and radiate the noise.

A thorough study of the cause of machine noise is important because the sound from major noise sources must be attenuated before reduction of the noise from secondary sources; such as surface vibration, will have any significant effect. The need for these investigations increases with increasing size and complexity of the machinery. In short, the larger and more complicated the machine, the more difficult it is to quiet.

Obviously, the most effective way of manufacturing quiet equipment is to incorporate good noise control techniques in the basic design stage. This is, of course, a concern of the design engineer and manufacturer. But if you, as a consumer, are familiar with certain principles governing noise in equipment operation you can know what to look for when making a purchase, or when attempting to control noise from appliances and equipment. Whether one is concerned about designing quiet equipment or faced with the problem of quieting an existing noisy device the following recommendations and corrective measures should be considered and applied wherever possible.

\section{REDUCE IMPACT OR IMPULSIVE FORCES}

Many machines and items of equipment are designed with parts that strike forcefully against other parts, producing noise. Often, this striking action, or impact, is essential to the machine's function. A familiar example is the typewriter - its keys must strike the ribbon and paper in order to leave an inked letter-impression. But the force of the key also produces noise as the impact falls on the ribbon, paper and platen.

Other common devices that produce impact noise include quick-acting cut-off valves found in washing machines and furnace humidifiers. The loud "thump" they often make can be startling, annoying, or in the case of some furnace controls, they can disturb sleep.

Several steps can be taken to reduce noise from impact forces. The particular remedy to be applied will be determined by the nature of the machine in question. Not all of the steps listed below are practical for every machine and for every impact-produced noise. But application of even one suggested measure can often reduce the noise appreciably. A knowledge of the principles underlying impact-noise reduction can also assist you in purchasing quieter equipment. 

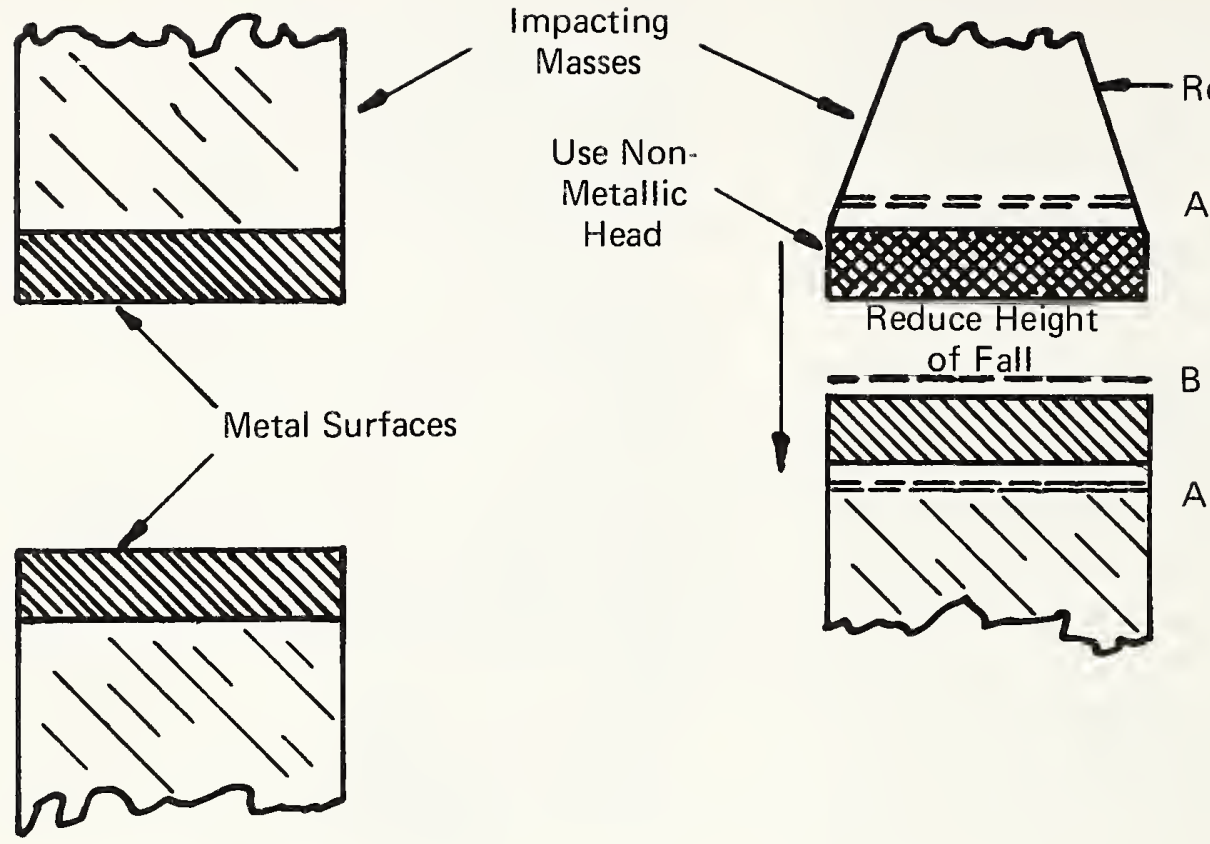

Fig. 3-1. METHODS OF REDUCING IMPACT FORCES TO LOWER NOISE RADIATION

a) Reduce weight, size or height of fall of impacting mass.

b) Cushion the impact by inserting a layer of shock-absorbing material between the impacting surfaces. (For example, insert several sheets of paper in the typewriter behind the top sheet to absorb some of the noise-producing impact of the keys hitting against the platen.) In some situations, you may insert a layer of shock-absorbing material behind each of the impacting heads or objects to reduce transmission of impact energy to other parts of the machine.

c) Whenever practical, one of the impact heads or surfaces should be made of non-metallic material to reduce "resonance or ringing" of the heads. Figure 3-1 shows the application of measures $a, b$, and c.

d) Substitute the application of a small impact force over a long time period for a large force over a short period to achieve the same result.

e) Smooth out acceleration of moving parts; apply accelerating forces gradually. Avoid high peak acceleration or jerky motion.

f) Minimize overshoot, backlash, loose play in cams, followers, gears, linkages, etc. This can be achieved by reducing the operational speed of the machine, better adjustment, or by using spring-loaded restraints or guides. Machines that are well made, with parts machined to close tolerances generally produce a minimum of such impact noise.

2. REDUCE SPEED IN MACHINES, AND FLOW VELOCITIES AND PRESSURES IN FLUID SYSTEMS

Reducing the speed of rotating and moving parts in machines and mechanical systems results in smoother operation and lower noise output.

Likewise, reducing pressure and flow velocities in air, gas and liquid 
circulation systems lessens turbulence resulting in decreased noise radiation.

(a) For quiet operation, fans, impellers, rotors, turbines, blowers, etc., should be operated at the lowest bladetip speeds that will still meet job needs.

(b) Use large-diameter low-speed fans rather than small-diameter
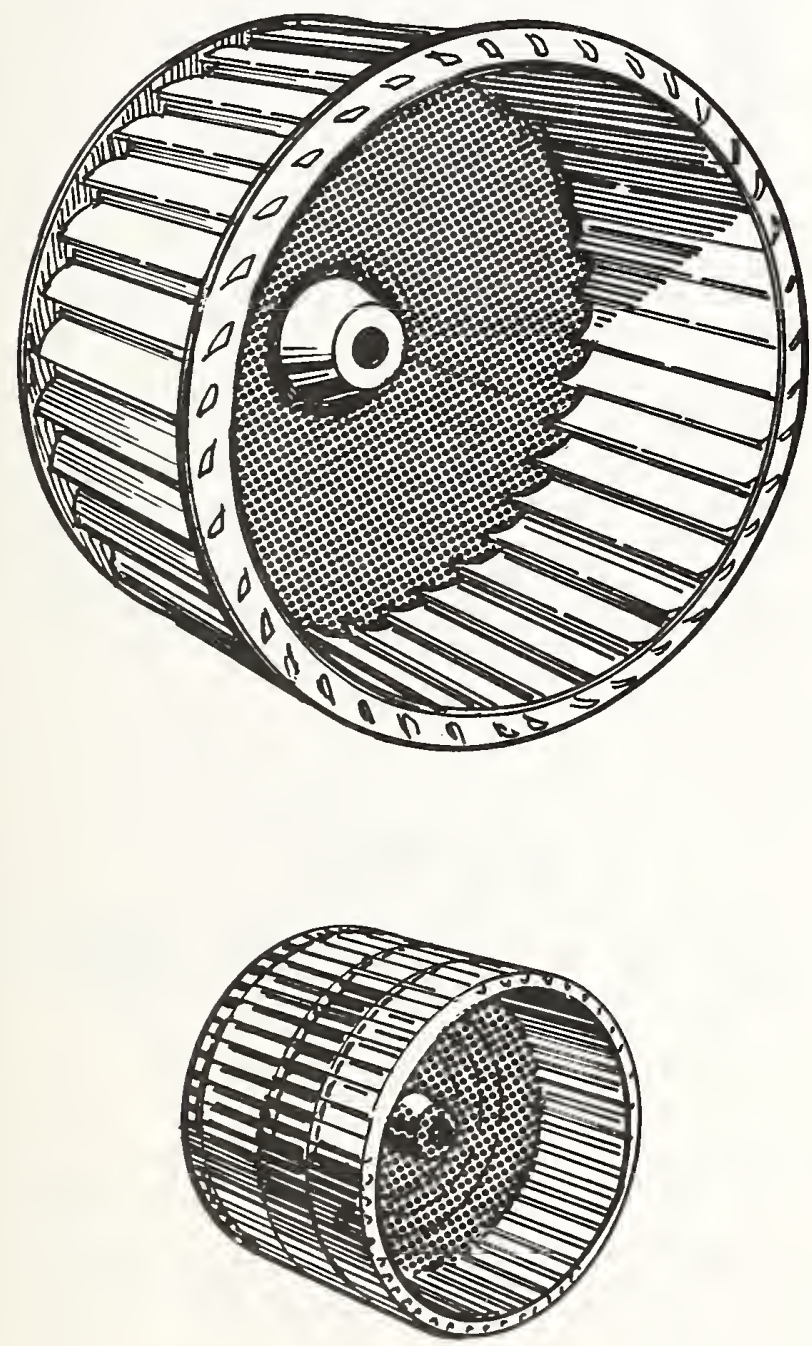

Squirrel Cage or Centrifugal Fans high-speed units for quiet operation. In short, maximize diameter and minimize tip speed.

(c) Centrifugal squirrel-cage type fans, sometimes used in furnaces or on exhaust ducts, are less noisy than vane axial or propeller type fans, a11 other factors being equa1. The two types of fans are illustrated in Fig. 3-2.
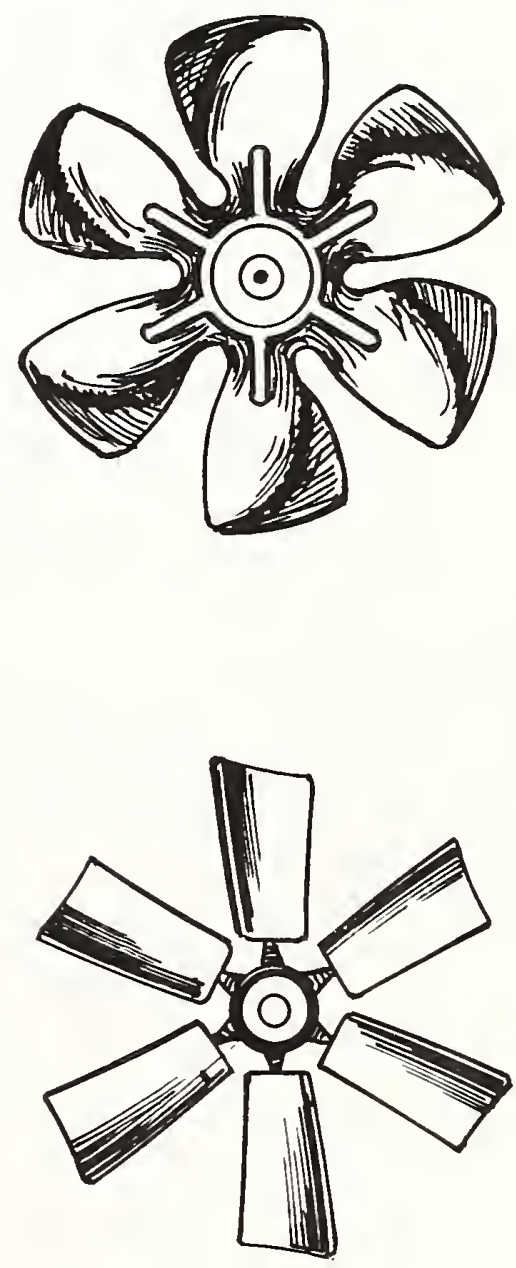

Propeller or Vaneaxial Fans

Fig. 3-2. FOR A GIVEN MASS FLOW, SQUIRREL CAGE FANS GENERALLY ARE LESS NOISY THAN PROPELLER TYPE FANS 
(d) Water pressure in individual home,

office, or apartment plumbing systems should be limited to 35 pounds per square inch (241 kilopascals) and flow velocities of the order of $6 \mathrm{ft} / \mathrm{sec}(2 \mathrm{~m} / \mathrm{sec})$ for quiet operation. A competent plumber can perform these adjustments.

(e) In air ventilation systems, a 50-percent reduction in air flow velocity may lower the noise output by 10 to $20 \mathrm{~dB}$, or roughly to $1 / 2$ to $1 / 4$ of the original loudness. Air flow velocities of $8-10 \mathrm{ft} / \mathrm{sec}(3 \mathrm{~m} / \mathrm{sec})$ as measured at a supply or return grille produce a low level of noise which usually is unnoticeable in most residential or office areas. In a given system, reduction of air flow velocity can be achieved by operating at lower motor or blower speeds, installing a greater number of ventilating grilles, or by increasing the cross-sectional area of the existing grilles. Your heating and air-conditioning contractor can make these adjustments and modifications.

One of the main sources of machinery noise is structural vibration caused by the rotation of poorly balanced parts such as fans, fly wheels, pulleys, cams, shatts, etc. Measures used to correct this condition involve the addition of counter weights to the rotating unit or the removal of some weight from the unit as indicated in Fig. 3-3. You are probably most familiar with noise caused by imbalance in the high-speed spin cycle of washing machines. The imbalance results from clothes not being distributed evenly in the tub. By redistributing the clothes, balance is achieved and the noise ceases. This same principle - balance - can be applied to furnace fans and other common sources of such noise. On some furnace blowers driven by a single belt, an unbalanced load may be applied if the blower pulley can move out of direct alignment with the motor pulley; if the load is applied symmetrically through a pair of belts driving pulleys on either side of the fan and the motor shafts, both shafts will tend to stay centered and the pulleys aligned, resulting in less noisy operation and reduced wear.

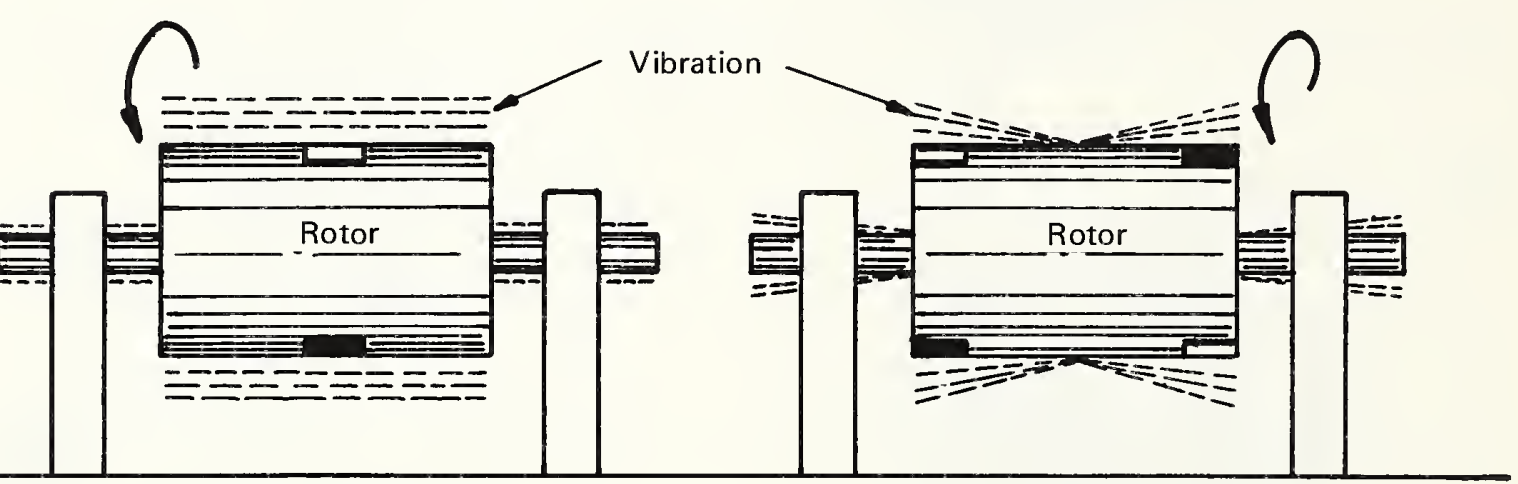

(a) Static Unbalance

(b) Dynamic Unbalance

Black blocks are heavy parts of rotor that cause vibration. White blocks are locations where counterweights must be placed to eliminate the vibration.

Fig. 3-3. EFFECTS OF STATIC AND DYNAMIC UNBALANCE ON ROTOR OPERATION

\section{REDUCE FRICTIONAL RESISTANCE}

Reducing friction between rotating, sliding or moving parts in mechanical systems frequently results in smoother operation and lower machine noise output.
Similarly, reducing flow resistance in air, gas and liquid distribution systems results in less noise radiation. In most cases, applying any one or a combination of the following corrective measures should provide a noticeable reduction in noise output. 
Reducing Resistance In Mechanical Systems

(See Fig. 3-4)

Lubricate: all rotating, moving, sliding, meshing or contacting parts should be lubricated with appropriate lubricant for quiet operation.

Align: Proper alignment of all rotating, moving or contacting parts results in less noise output. Maintain good axial and directional alignment in pulley systems, gear trains, shaft coupling, power transmission systems, bearing and axle alignment, etc.

Polish: Highly polished and smooth surfaces between sliding, meshing or contacting parts are required for quiet operation, particularly where bearings, gears, cams, rails, and guides, are concerned.

Balance: Static and dynamic balancing of rotating parts reduces frictional resistance and vibration, resulting in lower noise output.

Avoid eccentricity or out-of-roundness: Eccentricity or off-centering of rotating parts such as pulleys, gears, rotors, shaft/ bearing alignment causes vibration and noise. Likewise, out-of-roundness of wheels, rollers, and gears causes uneven wear, resulting in flat spots which generate vibration and noise.

Reducing Resistance In Air or Fluid Flow Systems (See Fig. 3-5)

The key advice to effective noise

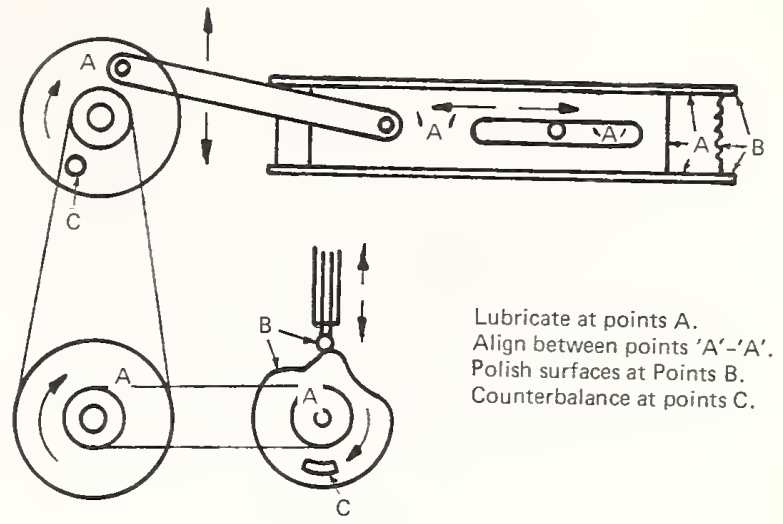

Fig. 3-4. REDUCING FRICTION OF ROTATING AND SLIDING PARTS TO DECREASE NOISE RADIATION

control in such systems is "streamline the flow". This holds true regardless of whether one is concerned with air flow in ducts or vacuum cleaners, or water flow in plumbing systems. Streamline flow is simply smooth, nonturbulent, low-friction flow, but it is the essential requirement for quiet operation of any type of fluid flow system.

The two most important factors which determine whether flow will be streamline or turbulent are flow velocity and the cross-sectional area of the flow path - that is, the pipe diameter. The rule of thumb for quiet operation is to use a lowvelocity, large-diameter pipe system to mieet a specified flow capacity requirement. However, even such a system can inadvertently generate noise if certain aerodynamic design features are overlooked or ignored. A system designed for quiet
NOISY DESIGN

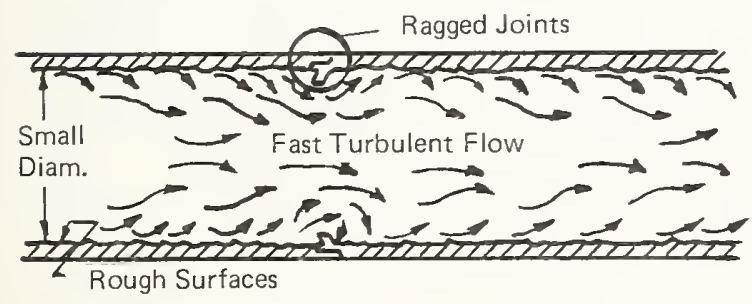

QUIET DESIGN

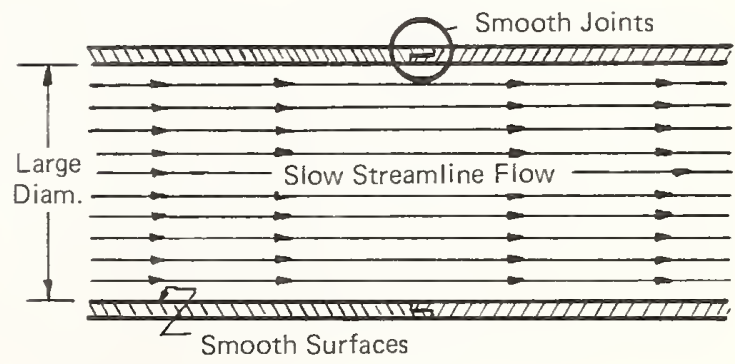

Fig. 3-5a. DESIGN OF QUIET FLOW SYSTEMS 

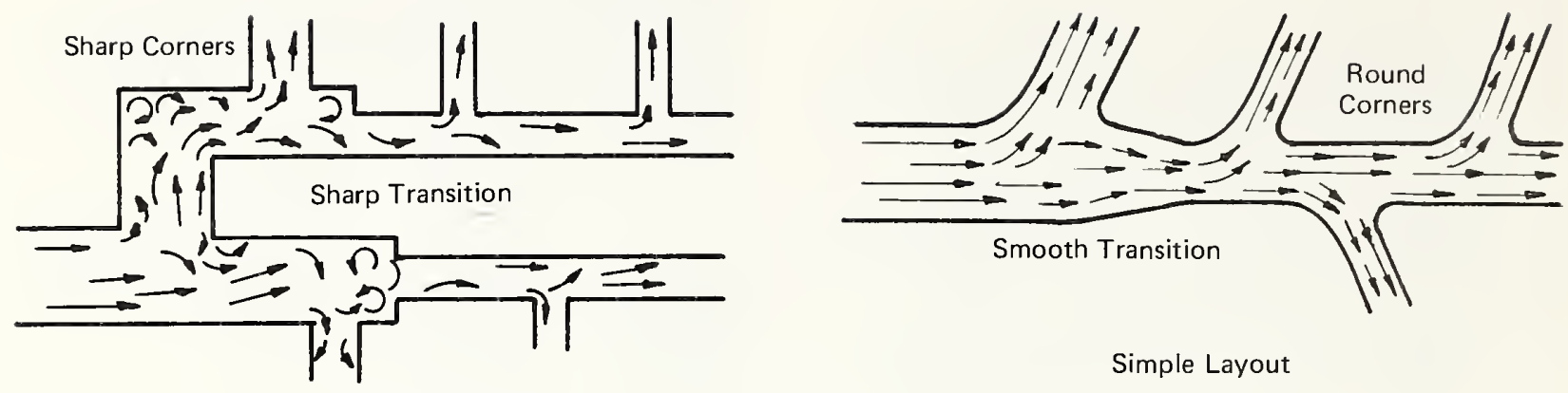

Complicated Layout

Simple Layout

Fig. 3-5b. DESIGN OF QUIET FLOW SYSTEMS

operation will employ the following features :

Low flow velocities: Low flow velocities avoid turbulence, one of the main causes of noise. Flow velocities should be of the order of 8 to 10 feet per second $(3 \mathrm{~m} / \mathrm{sec})$ in domestic forced-air heating and ventilation systems and plumbing systems for quiet operation.

Smooth boundary surfaces: Duct or pipe systems with smooth interior walls, edges, and joints generate less turbulence and noise than systems with rough or jagged walls or joints.

Simple layout: A well-designed duct or

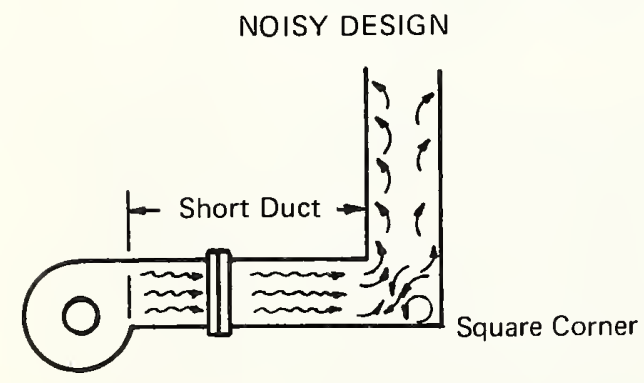

Turbulent Flow pipe system with a minimum of branches, turns, fittings and connectors is substantially less noisy than a complicated layout.

Long-radius turns: Changes in flow direction should be made gradually and smoothly. It has been suggested that turns should be made with a curve radius equal to about five times the pipe diameter or major cross sectional dimension of the duct.

Flared sections: Flaring of intake and exhaust openings, particularly in a duct system, tends to reduce flow velocities at these locations, often with substantial reductions in noise output.

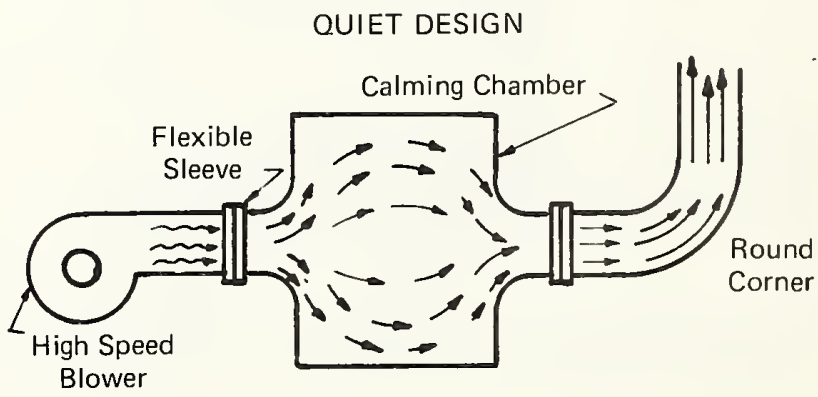

Smooth Flow

Fig. 3-5c. DESIGN OF QUIET FLOW SYSTEMS 


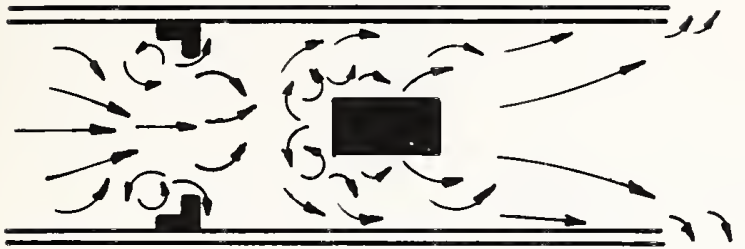

Turbulence Caused by Rectangular Devices

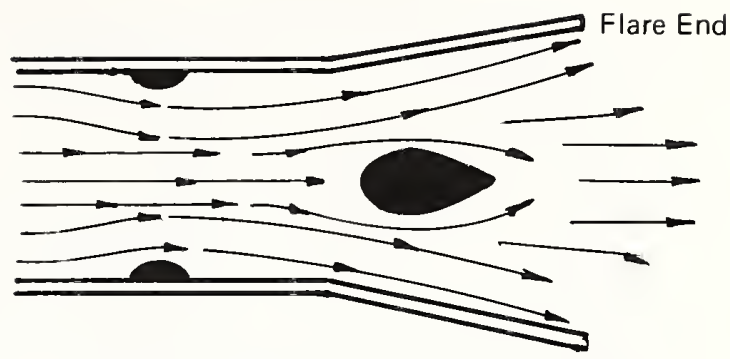

Remove or Streamline Objects in Flow Path

Fig. 3-5d. DESIGN OF QUIET FLOW SYSTEMS

Streamline transition in flow path:

Changes in flow path dimensions or cross-sectional areas should be made gradually and smoothly with tapered or flared transition sections to avoid turbulence. A good rule of thumb is to keep the cross-sectional area of the flow path as large and as uniform as possible throughout the system.

Remove unnecessary obstacles:

The greater the number of obstacles in the flow path, the more tortuous, turbulent, and hence the noisier the flow. Al1 other required and functional devices in the path such as structural supports, deflectors, and control dampers, should be made as small and as streamlined as possible to smooth out the flow patterns.

In other cases, parts with perforations, slots or other openings permit air leakage through or around the part as it moves, thus eliminating air pressure buildup, the chief cause of flexing and pulsation. Such action when there is no air leakage gives a popping sound - as the noise produced when squirting oil from an oil can. THE MACHINE

\section{ISOLATE VIBRATING ELEMENTS WITHIN}

In all but the simplest machines, the vibrational energy from a specific moving part is transmitted through the machine structure forcing other component parts and surfaces to vibrate and radiate sound often with greater intensity than that generated by the originating source itself.
For example, a water pump fastened to a side panel of a washing machine, as illustrated in Fig. 3-6, will cause the panel to vibrate and produce greater noise output.

The vibration generated by moving or rotating parts of a machine should be confined as close as possible to the area containing the source of vibration. In short, the vibrating unit must be isolated as much as possible from the structural frame or machine housing. The most effective method of vibration isolation involves the resilient mounting of the vibrating component on the most massive and structurally rigid part of the machine. All attachments or connections to the vibrating part in the form of pipes, conduits, shaft couplers, etc., must be made with flexible or resilient connectors or couplers otherwise the vibrational isolation afforded by the resilient mounts will be bypassed or shortcircuited. For example, pipe connections to a pump resiliently mounted on the structural frame of a machine should be made of resilient tubing, preferably as clcse to the pump as possible. Resilient pipe supports or hangers may also be required to avoid bypassing of the isolated system. In addition to these measures it is often good practice in the case of large machines or appliances such as washing machines, dryers, refrigerators or air conditioning equipment to set the entire machine on resilient mounts to prevent vibrational excitation of the supporting floor, and thus avoid radiation of noise into the area below or adjacent areas. These measures are illustrated in Fig. 3-7. 

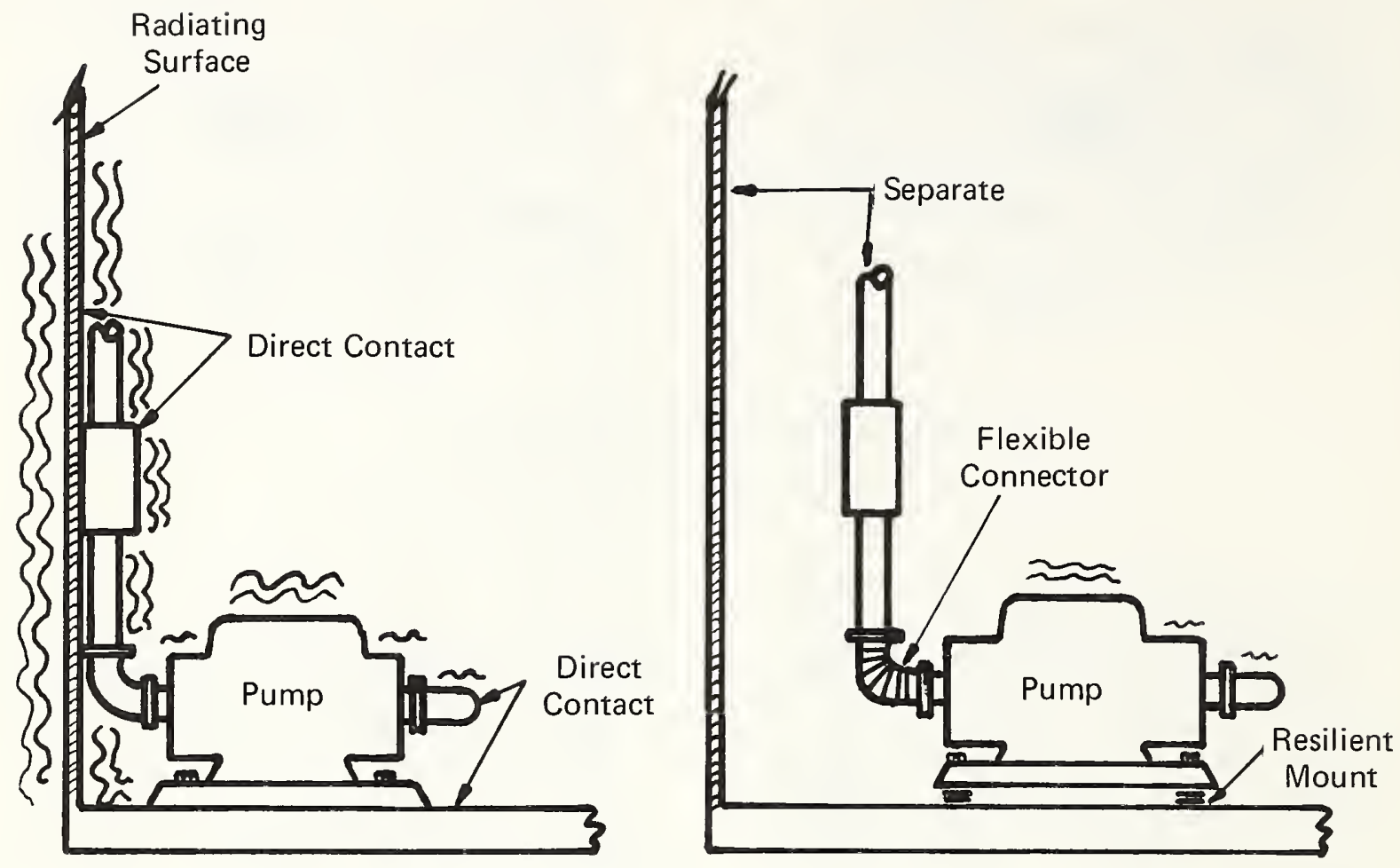

Fig. 3-6. ISOLATE LARGE RADIATING SURFACES FROM VIBRATING PARTS 


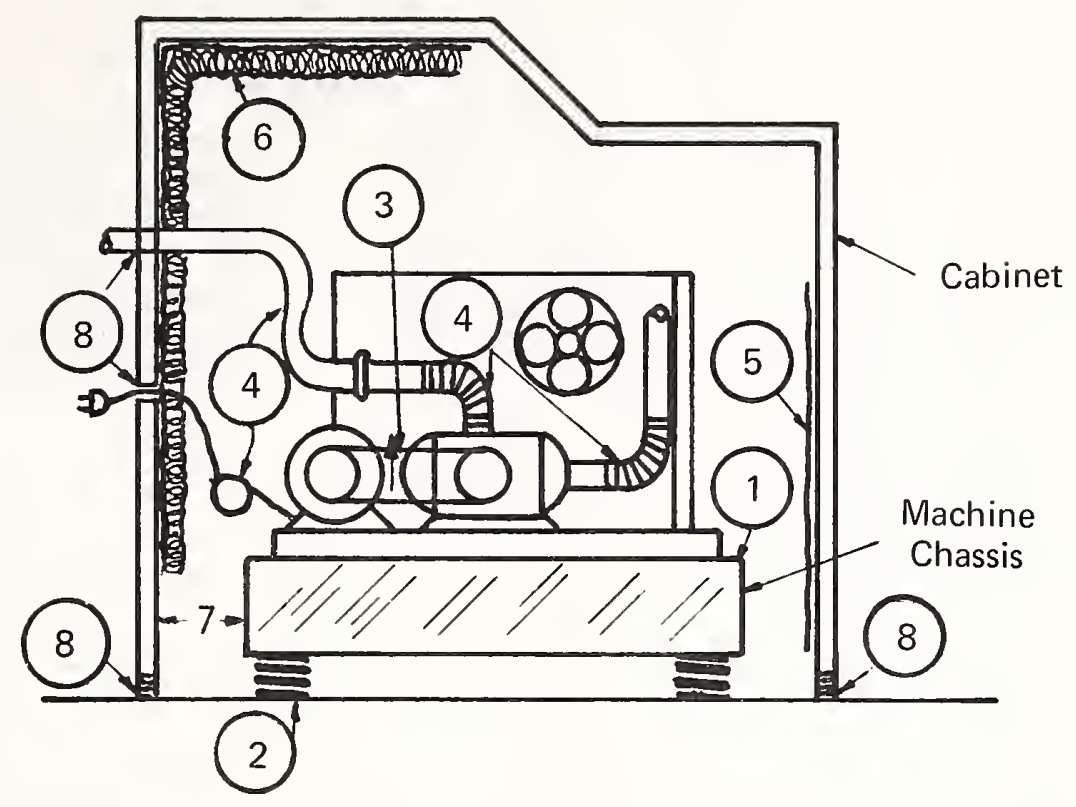

1. Install motors, pumps, fans, etc. on most massive part of the machine.

2. Install such components on resilient mounts or vibration isolators.

3. Use belt drive or roller drive systems in place of gear trains.

4. Use flexible hoses and wiring instead of rigid piping and stiff wiring.

5. Apply vibration damping materials to surfaces undergoing most vibration.

6. Install acoustical lining to reduce noise buildup inside machine.

7. Minimize mechanical contact between the cabinet and the machine chassis.

8. Seal openings at the base and other parts of the cabinet to prevent noise leakage.

Fig. 3-7. TECHNIQUES TO REDUCE THE GENERATION OF AIRBORNE AND STRUCTURE·BORNE NOISE IN MACHINES AND APPLIANCES 
Large Vibrating

Surfaces

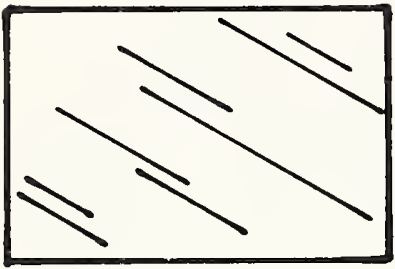

NOT THIS
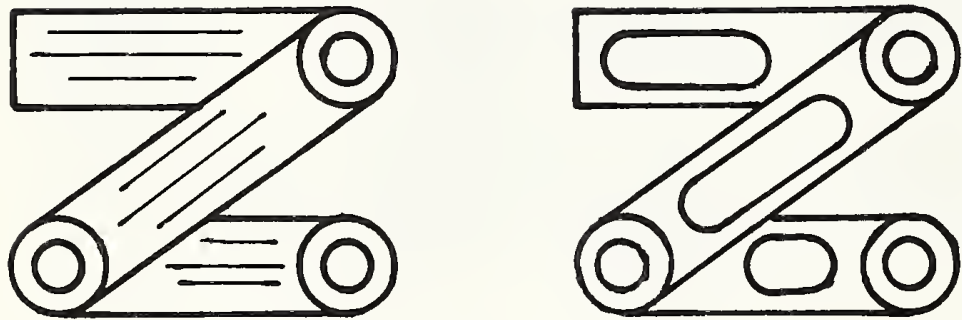

THIS

Fig. 3-8. REDUCE THE AREA OF VIBRATING SURFACES TO LOWER NOISE RADIATION

\section{3-8) \\ 6. REDUCE RADIATING AREA (See Fig.}

Generally speaking, the larger the vibrating part or surface, the greater the noise output. The rule of thumb for quiet machine design is to minimize the effective radiating surface areas of the parts without impairing their operation or structural strength. This can be done by making parts smaller, removing excess material, or by cutting openings, slots or perforations in the parts. For example, replacing a large vibrating sheet metal safety guard on a machine with a guard made of wire mesh or metal webbing might result in a substantial reduction in noise, because of the drastic reduction in surface area of the part.
OR

THIS

Reduce Size of Part or

Cut Out Excess Material
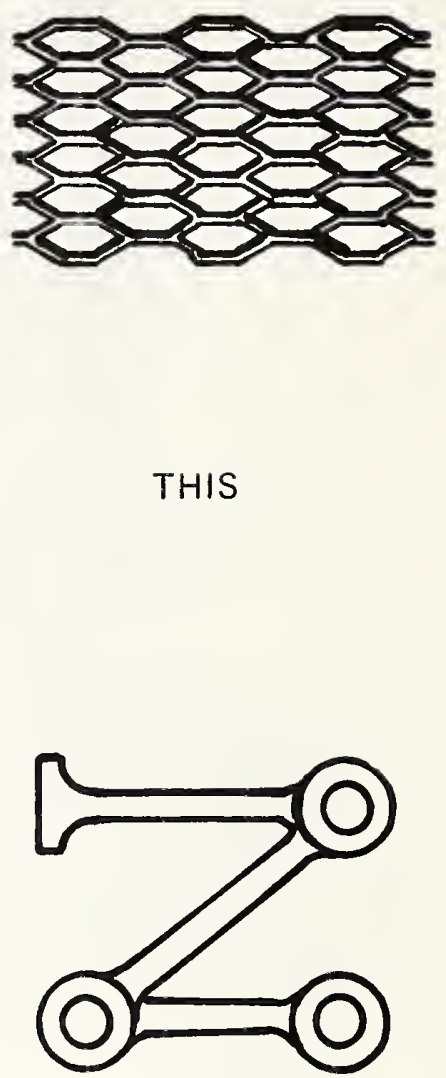
course, you would probably apply the materials to the inside surface to preserve the appearance of the appliance. Three basic types of vibration damping materials are available:

(a) liquid mastics which are applied with a spray gun and harden into relatively solid materials, the most common being automobile "undercoating";

(b) pads of rubber, felt, plastic foam, leaded vinyls, adhesive tapes or fibrous blankets which are glued to the vibrating surface;

(c) sheet metal viscoelastic laminates or composites which are bonded to the vibrating surface.

The type of material best suited for a particular vibration problem depends on a number of factors such as size, mass, vibrational frequency and operational function of the vibrating structure.
However the following guidelines should be observed in the selection and use of such materials to maximize vibration damping efficiency:

(a) Damping materials should be applied to those sections of a vibrating surface where the most flexing, bending or motion occurs. These usually are the thinnest sections.

(b) For a single layer of damping material, the stiffness and mass of the material should be comparable to that of the vibrating surface to which it is applied. This means that single layer damping materials should be about two or three times as thick as the vibrating surface to which they are applied.

(c) Sandwich materials made up of metal sheets bonded to mastic ("sheet-metal viscoelastic composites") are much more

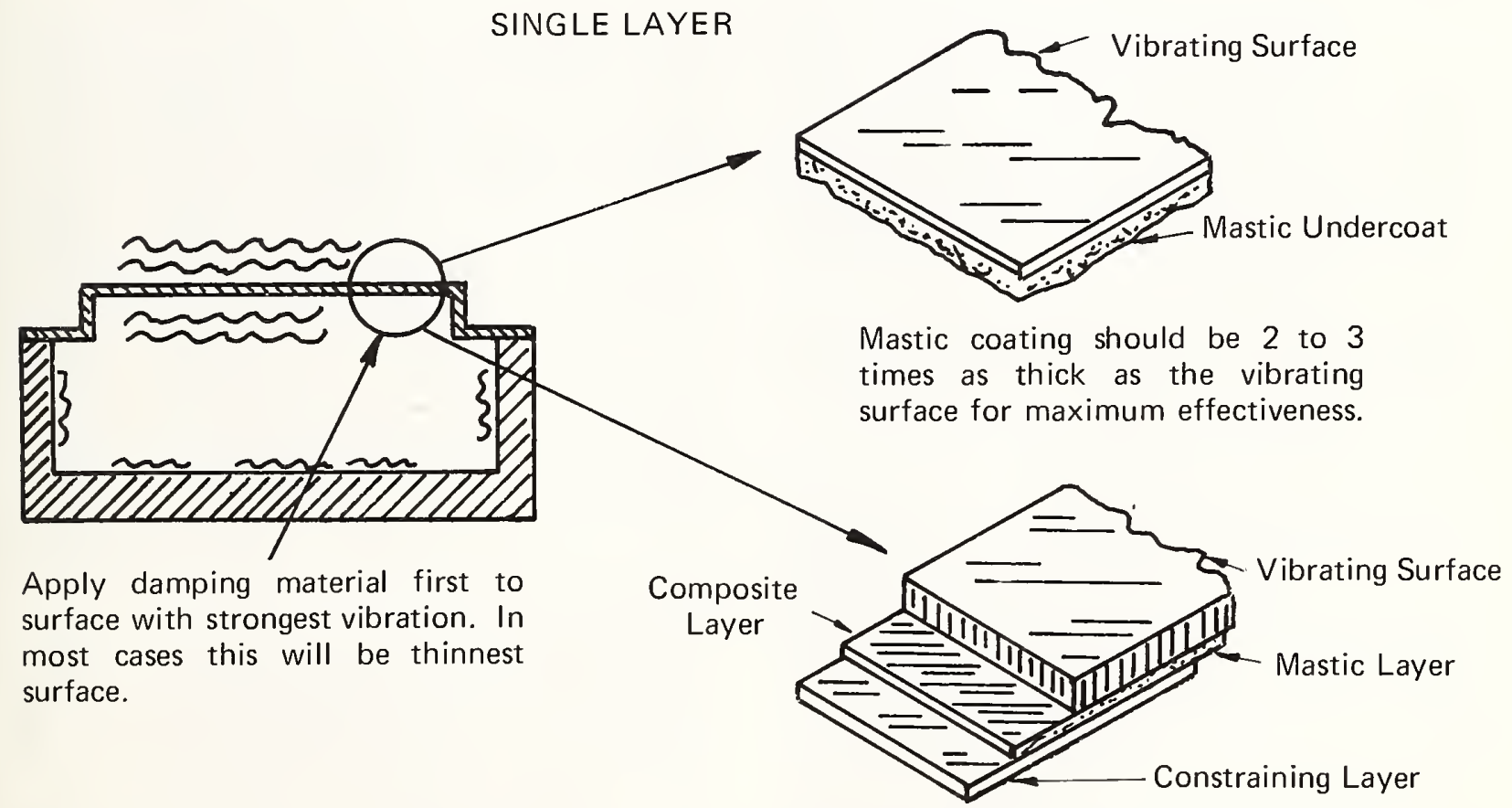

For effective vibration damping, each layer should be about $1 / 3$ as thick as the vibrating surface.

Fig. 3-9. REDUCING VIBRATION WITH DAMPING MATERIALS 
Openings Around

Pipes, Conduits, Etc.

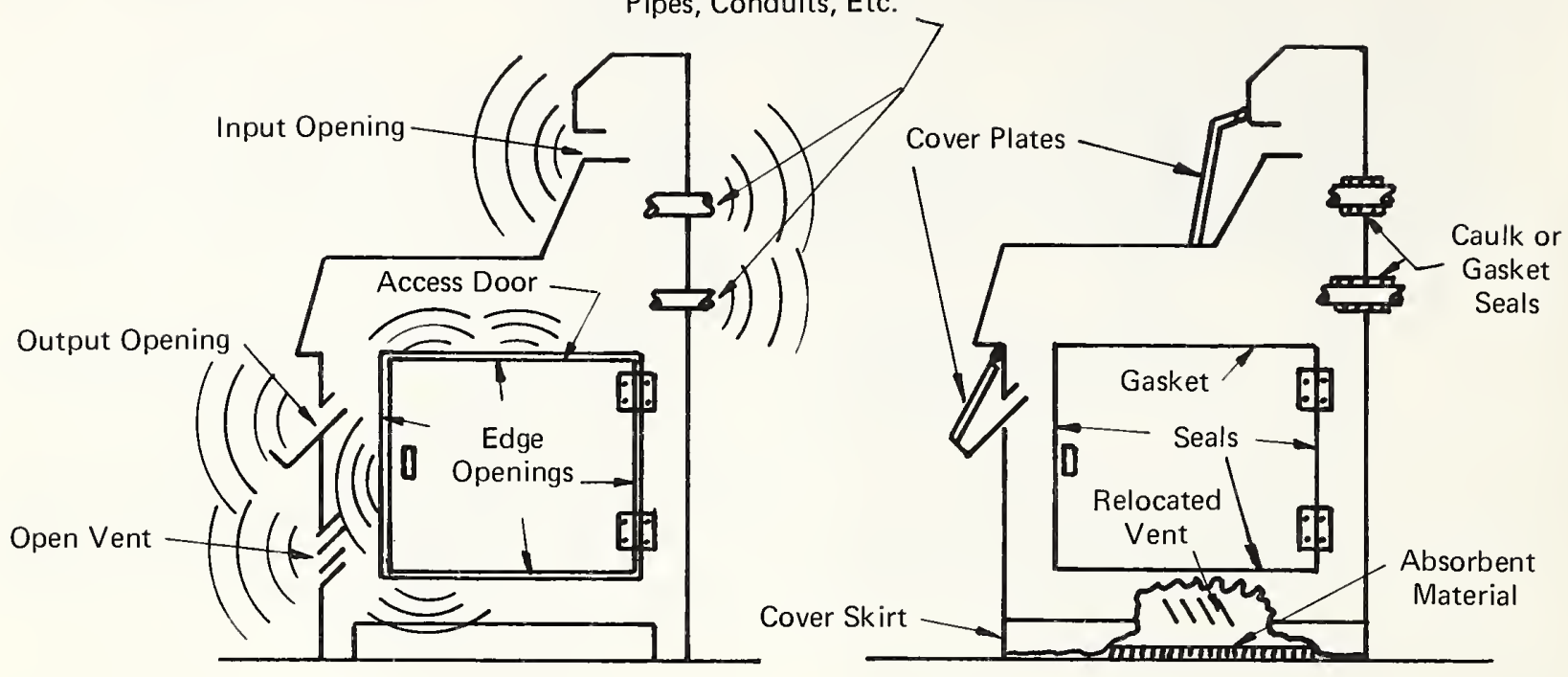

Fig. 3-10. TECHNIQUES FOR REDUCING THE RADIATION OF NOISE FROM OPENINGS IN A MACHINE

effective vibration dampers than single layer materials; the thicknesses of the sheet metal constraining layer and the viscoelastic layer should each be about one-third the thickness of the vibrating surface or panel of the appliance to which they are applied.

8. REDUCE NOISE LEAKAGE FROM THE INTERIOR OF THE APPLIANCE

(See Fig. 3-10)

In many cases machine cabinets can be made into rather effective soundproof enclosures through simple design changes, and the application of some sound absorbing treatment. Substantial reductions in noise output may be achieved by adopting some of the following recommendations, especially if some of the vibration isolation techniques discussed earlier are also used.

(a) Seal or cover all openings

(i) All unnecessary holes or cracks, particularly at joints, should be caulked or closed off.

(ii) All electrical or plumbing penetrations of the housing or cabinet stiould be sealed with rubber gaskets or a suitable non-setting caulk.

(iii) If practical, all other functional or required openings or ports which radiate noise should be covered with lids or shields edged with soft rubber gaskets to effect an airtight seal. For example, the lid of a top-loading washing machine may be fitted with a rubber gasket or with rubber strips to prevent the escape of the noise from the agitator.

(iv) Other openings required for exhaust, cooling or ventilation purposes should be equipped with mufflers or acoustically lined ducts.

(v) Relocate or direct openings away from the operator and other people.

(b) Apply acoustical materials

(i) Install sound absorbent lining on inner surfaces of cabinets to reduce noise 
buildup in the reverberant cavities. (We will discuss reverberation further in Section 3 under Factors Affecting the Travel of Sound)

\section{(ii) Apply vibration damping material to the inner surfaces of all vibrating panels.}

\section{REPLACING APPLIANCES}

This advice is, of course, of little comfort if you find yourself the owner of a new, noisy appliance. In due time, though, it will need to be replaced, and this is an opportunity for choosing an effectively QUIETED device. However, some minor changes in accessories can reduce noise problems:

(a) Replace plastic blender containers with containers made of heavy glass. Often both alternatives are available; glass costs very little more.

(b) Substitute rubber or plastic trash cans for noisy metal cans. Some laminated metal trash cans have been made for industrial use; the new concern with quiet may make them desirable for ordinary domestic use.

(c) Use wood or fiberboard cabinets instead of the noisier metal units.

(d) Select sliding or folding closet doors made of wood or laminate instead of metal doors which are apt to rattle or vibrate when moved.

(e) High velocity air flowing over the corrugated surfaces inside a hose often generates a whistling noise. Replacing the corrugated hose of a vacuum cleaner with a smooth surfaced hose will reduce this noise.

(f) Replace small high-speed floor fans or exhaust fans with larger, slower-moving units.

(g) Substitute a belt-driven furnace blower operated by a resiliently-mounted motor for a motor-coupled blower.
After you have tried all possible ways of controlling the noise at the source, your next line of defense is to set up barriers or other devices in the transmission path to block or reduce the flow of sound energy before it reaches your ears. This can be done in several ways:

you can absorb the sound along the path,

you can deflect the sound in some other direction away from you by placing a reflecting barrier in its path, and

you can contain the sound by placing the source inside a sound- insulating box or enclosure.

Selection of the most effective technique will depend upon various factors such as the size and type of source, intensity and frequency range of the noise, and the nature and type of environment. In addition one should have a basic understanding of the characteristics and behavior of sound that influence its propagation or travel through the air.

As we mentioned before, a sound wave traveling through the air creates a succession of compressions -- high-pressure waves -- followed by rarefactions, or low-pressure waves. These waves move away from the source in an alternating manner much like the spreading of ripples in a pond into which a pebble was dropped, as illustrated in Fig. 3-11. The distance between adjacent peaks of the high pressure waves is the wavelength of the sound. The frequency of the sound " $f$ " and its speed of trave1 "c" determine $L$ the wavelength. These three quantities are related by the equation $c=f L$. Since the speed of sound in air is relatively constant for all frequencies, the wavelength decreases as frequency increases. Sound travels at a speed of about 1130 feet $(345 \mathrm{~m})$ per second. Therefore, the wavelength for a $100 \mathrm{~Hz}$ tone would be approximately 11 feet $(3.45 \mathrm{~m})$ whereas for a $1000 \mathrm{~Hz}$ tone it would be about 1.1 feet $(34.5 \mathrm{~cm})$. As we shall see, both frequency and wavelength affect the propagation of sound in air and the manner in which it interacts with the environment.

\section{FACTORS AFFECTING TRAVEL OF SOUND}

\section{Absorption: Soaking Up Sound}

We know from experience that air 


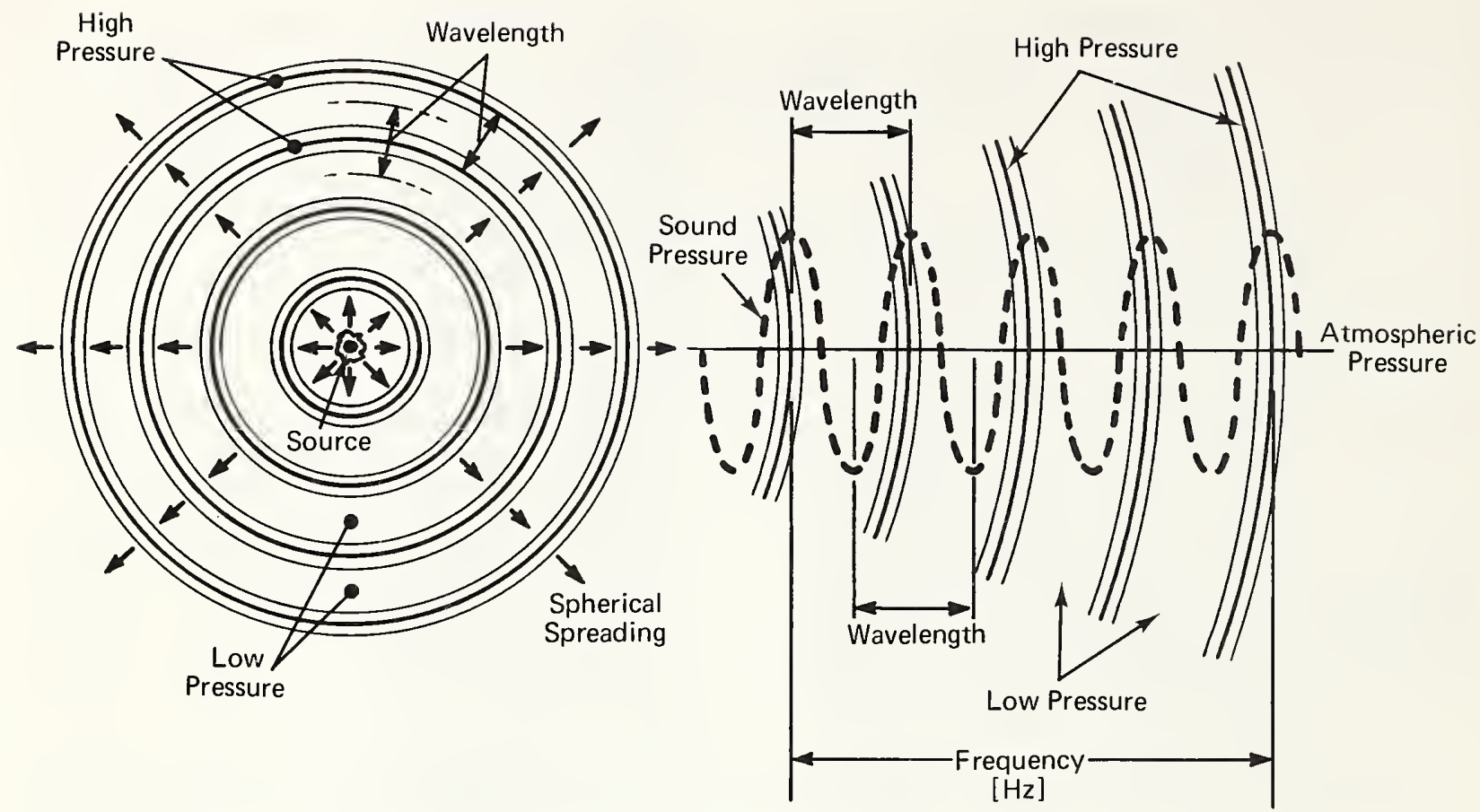

Fig. 3-11. PROPERTIES OF SOUND WAVES

absorbs sound, especially high-pitched sounds. That is why we hear only the low-pitched roar of exhaust noise from a high-flying jet plane and not the shrill high-pitched turbine noise we normally hear at the airport when the plane takes off. The low-pitched rumble of distant thunder sounds almost harmless compared to the sharp terrifying crack of thunder when heard close by. The high-pitched components of a thunderclap are absorbed by the air before they reach a distant listener.

Air absorbs high-pitched sounds more effectively than low. However, if the distance between a source and a listener is great enough, low-pitched sounds also will be absorbed appreciably by the air. For example, we might not hear any sound at a11 from a jet plane flying at an altitude so great that only its vapour trail is visible.

With the exception of very large auditoriums, convention halls, or sport arenas, the absorption of sound by air within typical buildings or rooms is negligible. However, in most buildings and rooms a significant degree of sound absorption can be achieved through proper furnishings. Because of their soft porous qualities, such materials as draperies and carpeting are excellent sound absorbers. Upholstered furniture and bed furnishings also have good sound absorbing characteristics. However, the installation of acoustical tile on ceilings in rooms, corridors and staircases is a practical method of controlling noise along its path of travel. Depending on the type of installation, and the frequency distribution of the noise, acoustical ceilings can absorb from 50 to $80 \%$ of the sound energy that strikes the surfaces. A soft, thick carpet with felt padding placed on the floor will absorb about 50 to $60 \%$ of airborne sound energy striking it. In addition, it will muffle impact and footstep noise very effectively. Pleated drapery will absorb about as much sound energy as carpet for the same amount of surface coverage. With these materials on ceilings, floors and walls, much of the sound energy is absorbed along the path before it can cause annoyance in other areas.

When sound waves strike such materials, they penetrate into the pores and air pockets of the material. There they bounce around haphazardly causing air in the pores and the fibers of the material to 
vibrate; this motion dissipates the sound energy in the form of heat because of frictional resistance between air molecules and fibers.

Drapery and carpeting absorb high-frequency sounds much more effectively than low. Because of their long wavelengths, low-frequency sound waves require materials that are many times thicker and have much larger pores and air pockets than those used for absorbing high-pitched sounds. For efficient absorption of low frequency sounds, the thickness, openings and pores of the acoustical material should bear the same proportion to the wavelengths of these sounds as the thickness, openings and pores of absorbers for high-frequency sounds bear to their respective wavelengths. Unfortunately, materials for absorbing low-frequency sounds are somewhat impractical in a house because of their large size and bulkiness.

Although sound waves generally keep on traveling in the direction in which they originally started, high-frequency sounds tend to follow narrower and more beam-like paths than do low-frequency sounds. We have noticed how highly directional the sound of a "hiss" or "psst", or a whistling tea kettle is compared, for example, with the rumbling sound of a washing machine. Low-frequency sounds tend to spread out uniformly in all directions from the noise source because of their long wavelengths.
The assumption that sound travels in straight lines is valid only for wavelengths that are short compared to the dimensions of open spaces, rooms or passage ways. Sound waves travel in straight lines until they encounter some obstacle in their path. If the obstacle is small compared to the wavelength of the sound, the wave front will hardly be disrupted.

2. Sound Reflection: Bouncing of Sound

When a sound wave strikes a solid flat surface that is large compared to the wavelength of sound, the wave is reflected similar to the way a sunbeam is reflected from a window pane. As sound waves strike a flat surface, they rebound at an angle equal to the one at which they originally struck the surface, as shown in Fig. 3-12.

A listener exposed to both the direct waves and reflected waves from a distant sound source will perceive the direct wave to be much louder than the reflected wave. At one time or another, you might have noticed that an echo of a person's handclap or a yell in a large hall or canyon seemed to be much less intense than the original sound. This reduction in loudness of the reflected wave or echo is due to the longer distance it has traveled, and also to the loss of energy due to absorption by reflecting surfaces and perhaps the air.

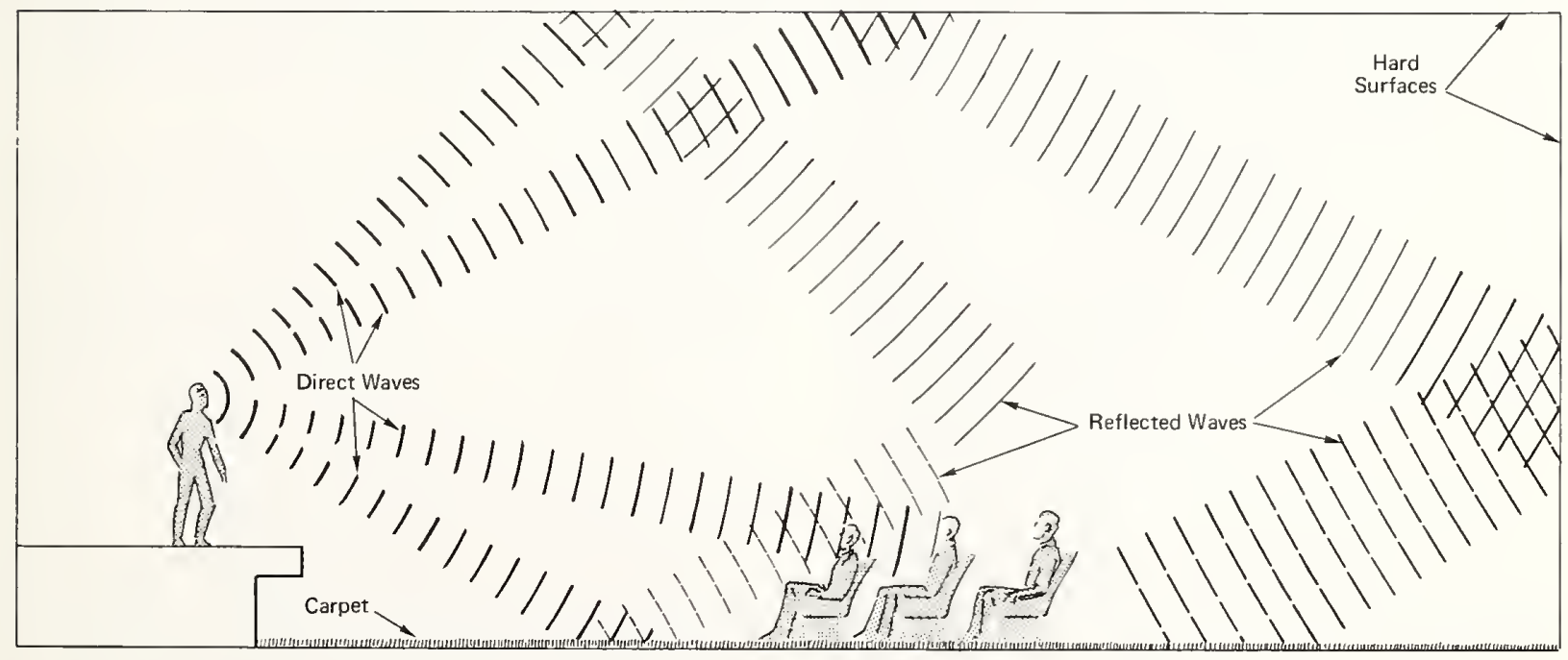

Fig. 3-12. PROPAGATION OF DIRECT AND REFLECTED SOUND WAVES IN A ROOM 
3. Reverberation: Reflection of Sound

Multiple

However, in a reverberant space of small dimensions, the reflected and direct sourid waves tend to merge to produce a sound that is louder than the direct sound alone. If successive reflections follow one another within a time interval of less than 35 milliseconds, the ordinary person perceives no echo. This corresponds to a spacing between successive reflections of less than about 35 feet $(10 \mathrm{~m})$. This type of sound reflection frequently is not only desirable but can be put to practical use. When properly used it will enhance the sound of music, or, as in the case of a band shell, it will reinforce the music reaching the audience.

You, perhaps, have taken advantage of this phenomenon, if you have mounted your stereo loudspeakers on a wall or in the corners of a room to get greater sound intensity for the same setting of the volume control.

Have you ever noticed upon entering a bare, unfurnished room how much louder your voice appears to be? The merging of the reflected waves builds up the sound level. This effect, which is called reverberation, is most prominent in rooms with hard reflecting surfaces such as a typical bathroom with its ceramic tile and plaster surfaces.

This phenomenon, more so than any other, gave birth to the so-called BATHROOM BARITONE. Rooms like the bathroom, which are highly reverberant, would be described as being acoustically live. Many basement areas and recreation rooms would fall in this category. Bedrooms and living rooms, on the other hand, would be considered as acoustically dead because of the large amounts of sound absorbent materials normally found in these rooms. Obviously, the Bathroom Baritone rarely performs in such rooms because his powerful voice suddenly would sound weak or dead, just like the environment.

Highly reverberant rooms tend to be excessively noisy. The most effective method of dealing with this problem is to install a large amount of sound absorbent treatment in the form of carpeting, drapery, upholstered furniture or an acoustical ceiling. As a rule of thumb, if the sound of a person's handclap or a shout in a particular rocm takes longer than a second or two to die away, the room is too reverberant, and requires acoustic treatment.

Although excessive reverberation of sounds in most instances is a problem associated with the interior of buildings or rooms, it can occur as well out of doors, The most common example is the reverberant echoing and rumble of thunder in mountainous areas. The high noise levels caused by the reverberation of traffic noise between tall buildings in major cities is familiar to all of us. The court areas of $U$ shaped apartment buildings tend to be excessively reverberant and noisy, particularly if they are used as recreation areas or if they face traffic arteries. Very little can be done to remedy problems of natural origin; however, man-made problems can be avoided through proper planning in the design stage. For example, a U shaped court yard should not front on a traffic artery.

of Sound

4. Diffraction: Bending or Squeezing

When a sound wave encounters an obstacle or an opening which is comparable in size to its wavelength, the sound will bend around the obstacle or squeeze through the opening with little loss of energy, as shown in Figs. 3-13a, 3-13b. This action is knowr as diffraction. Diffraction occurs when sound waves strike the edge of a solid barrier. This edge acts as a focal line from which a new train of waves is generated. These new waves which spread out into the area behind the barrier are of the same frequency as the original waves but lower in intensity. This explains why we can hear traffic noise from a busy highway even though it is hidden from view by a large building or a hill.

With regard to sound passing through smal1 openings, you may have noticed how readily sound passes through a door that is slightly ajar. With the conventional practice of installing doors with a $1 / 2$ to 3/4 inch (13-19 mm) air space at the bottom to provide for the circulation of return air, it is not surprising that we get no privacy even when the door is closed. The amount of sound energy that passes through a small hole or hairline crack in a wall is far greater than one would predict based on the size of the crack. This points out how important it is to caulk or seal all cracks or openings in walls, doors, etc., which separate areas where privacy is desired.

Inside buildings, the combined effects of diffraction and reflection permit sound 


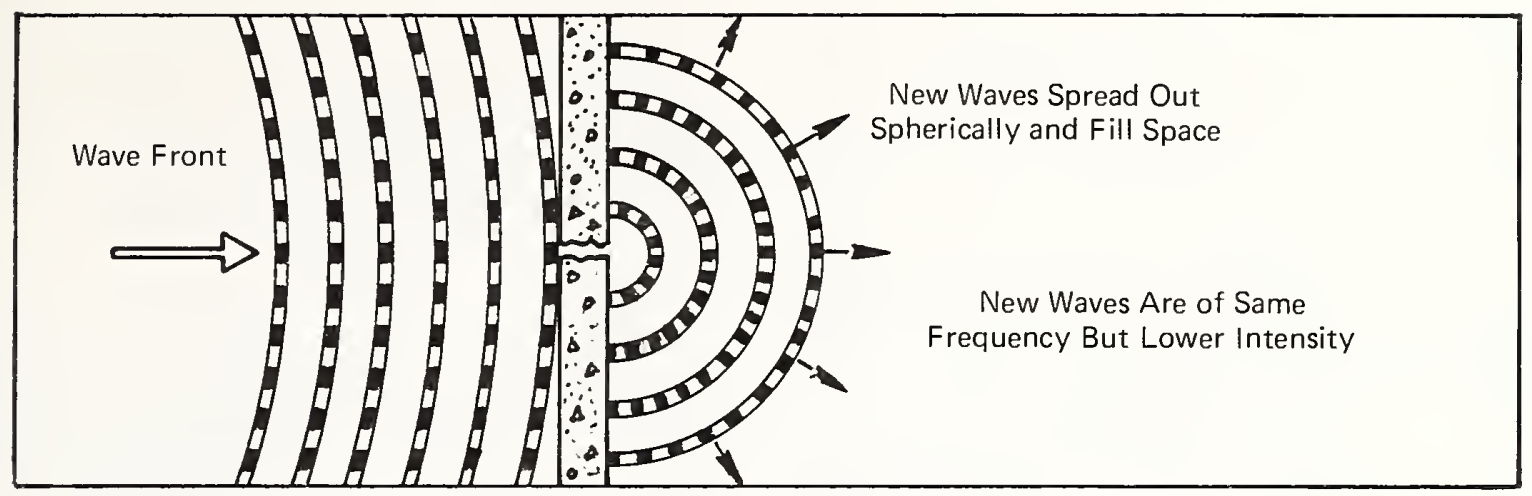

Fig. 3-13a. DIFFRACTION OF A SOUND WAVE PASSING THROUGH A SMALL HOLE

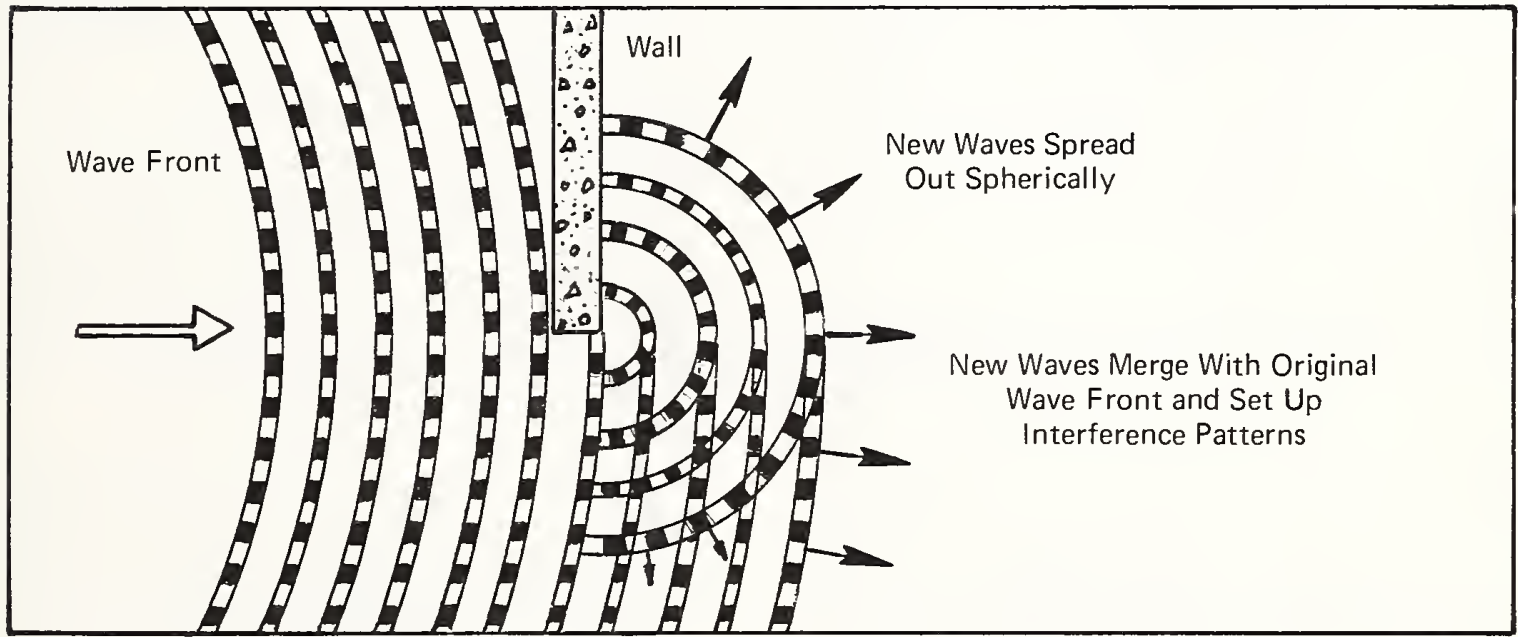

Fig. 3-13b. DIFFRACTION OF A SOUND WAVE AT THE EDGE OF A WALL

to trave1 along winding corridors, up staircases and through duct systems with surprising ease.

\section{"QUIETING" IN THE PATH}

1. Separate the Noise Source and Receiver As Much As Possible

In open spaces, sound coming from a point source, which is defined as one that radiates sound uniformly in all directions, tends to spread out in a wave front that is spherical in shape. The power at any point away from the source drops in proportion as the surface area of a sphere centered at the source increases. For each doubling of distance from the source, the surface area increases fourfold. Consequently, for each doubling of the distance, the power drops to one-fourth or the power level drops by $6 \mathrm{~dB}$.

Any source that is located at a distance that is large compared to the size of the source acts to the observer like a point source along a direct line between him and the source, even though the source itself may be relatively large and may radiate more sound in some directions than in others. Thus, a power mower located at a 
distance of 100 feet $(30 \mathrm{~m})$ and a large noisy factory located at a distance of $1 / 4$ mile $(0.4 \mathrm{~km})$ from the 1istener could both be corisidered as point sources so far as the listener is concerned.

Increasing your distance from a source of noise is a practical means of noise abatement, if you can manage it. If you can quadruple your distance from a point source you will have succeeded in lowering its loudness by at least one-half or roughly 12 $\mathrm{dB}$. However, if you have to contend with an extended or a line source such as a railroad train, the noise level drops by only $3 \mathrm{~dB}$ for each doubling of distance from the source. The main reason for this lower rate of attenuation is that line sources radiate sound waves that are cylindrical in shape. The surface area of such waves only increases twofold for each doubling of distance from the source. However, when the distance from the train becomes comparable to its length, the noise level will begin to drop at the rate of $6 \mathrm{~dB}$ for each subsequent doubling of distance. Figures 3-14a and 3-14b illustrate how the noise levels drop as distance increases from both types of noise sources.

Indoors, the noise level generally drops only from 3 to $5 \mathrm{~dB}$ for each doubling of distance in the near vicinity of the source. However, further from the source, reductions of only 1 or $2 \mathrm{~dB}$ occur for each doubling of distance, due to the reflections of sound off hard wall and ceiling surfaces.

\section{Use Sound Absorbing Materials}

Sound absorbing materials such as acoustic tile, carpets, and drapes placed on ceiling, floor or wall surfaces can reduce the noise level in most rooms by about 5 to $10 \mathrm{~dB}$ for high-pitched sounds, but only by 2 or $3 \mathrm{~dB}$ for low-pitched sounds. Unfortunately, such treatment provides no protection to an operator of a noisy machine who is in the midst of the direct noise field. For greatest effectiveness, sound absorbing materials should be installed as close to the noise source as possible.

If you have a small or limited amount of sound absorbing material and wish to make the most effective use of it in a noisy room, the best place to put it is in the upper trihedral corners of the room, formed by the ceiling and two walls. Due to the process of reflection, the concentration of sound is greatest in the trihedral corners of a noisy room. Therefore, the acoustic material which absorbs a fixed percentage of the sound energy that strikes its surface will remove a greater amount of sound energy when placed in such corners

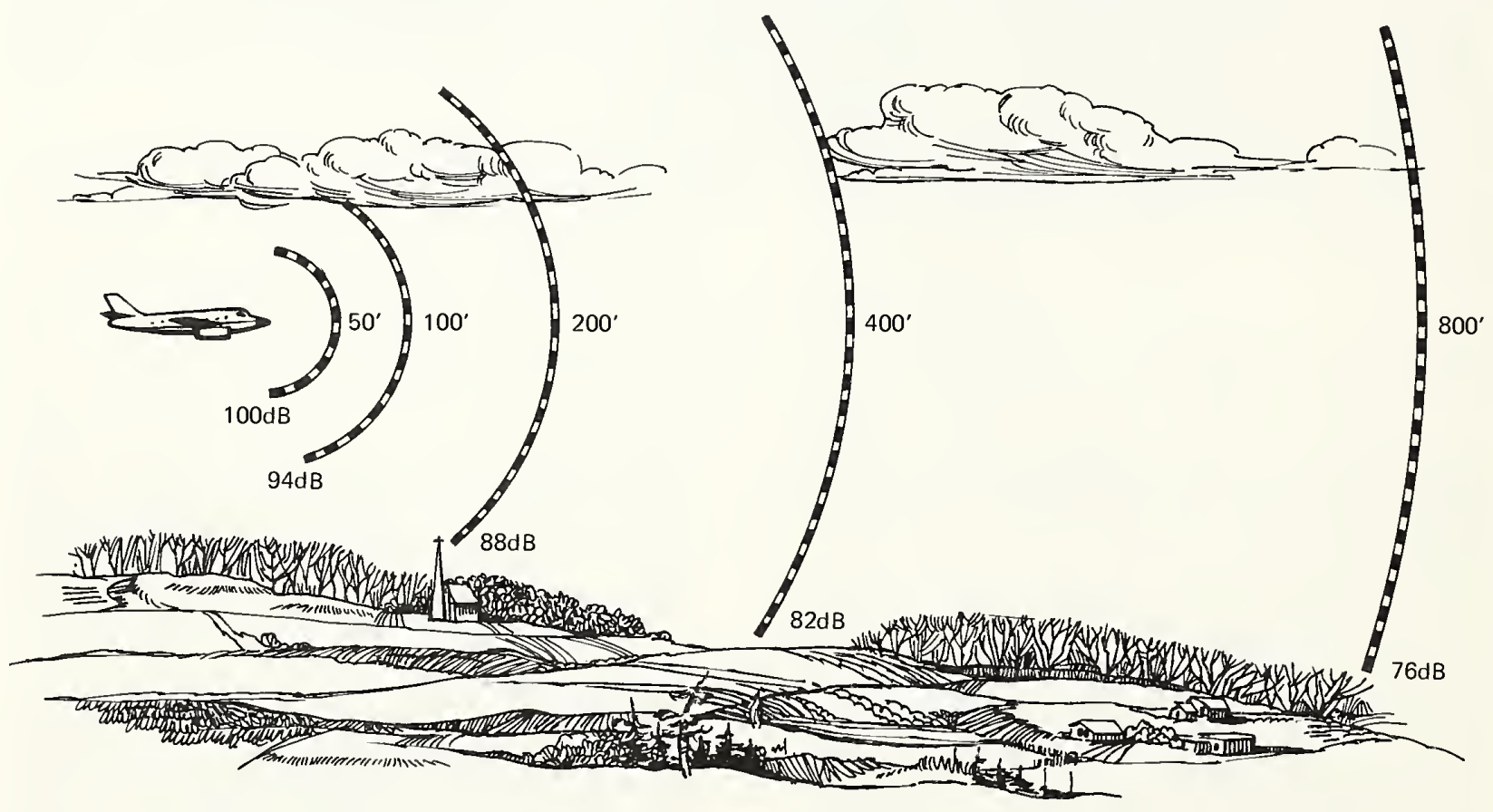

Fig. 3-14a. REDUCTION IN NOISE LEVEL WITH DISTANCE FROM A POINT SOURCE 


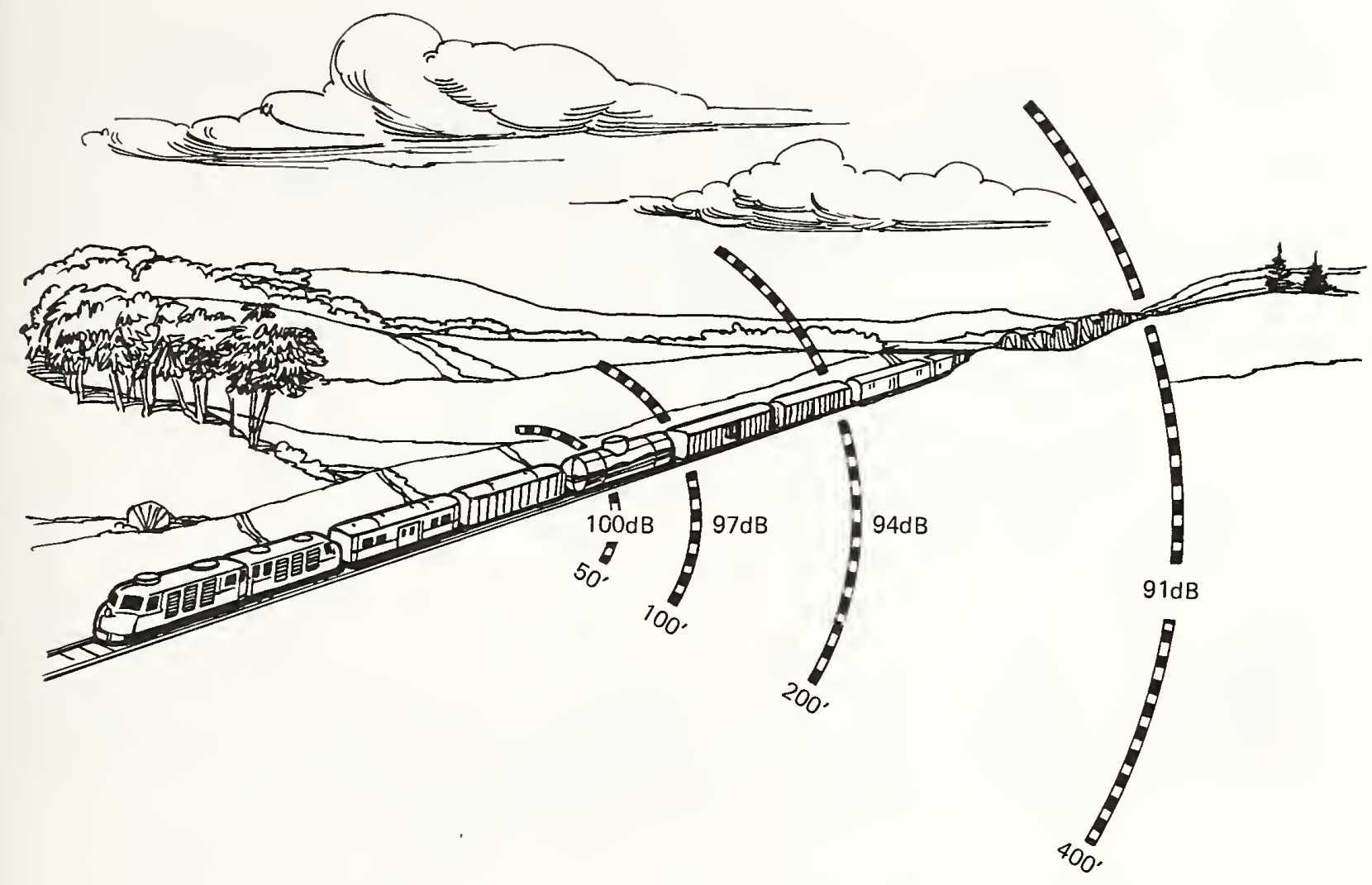

Fig. 3-14b. REDUCTION IN NOISE LEVEL WITH DISTANCE FROM A LINE SOURCE

than sowewhere else in the room. The upper corner locations also protect the ligh-weight fragile material from damage.

However, because of their light weight and porous nature, acoustical materials are ineffectual when applied to wall or ceiling surfaces to prevent the transmission of either airborne or structure-borne sound from one room to another. In other words, if you can hear people walking or talking in the room or apartment above, installing acoustical tile on your ceiling will not reduce the noise transmission. In most cases, the installation of a soft, thick carpet on the floor above will reduce the transmission of footstep noise substantially. However, reduction of both footstep and conversational noise might require the addition of either a floating floor or a resiliently suspended gypsum board ceiling to the existing floor-ceiling construction.

3. Use Sound Barriers and Deflectors

Placing barriers, screens, or deflectors in the noise path can be an effective way of reducing noise transmission, providing that the barriers are large enough in size, and depending upon whether the noise is high-pitched or low-pitched. Wood or metal panels lined with acoustical materials and placed in front of or around some noisy machine might attenuate the noise reaching a worker on the other side by about 10 to $15 \mathrm{~dB}$ if the noise is high-pitched. For example, in a room relatively free of echoes, the noise from a card-punch machine, which has a fundamental frequency of about $3000 \mathrm{~Hz}$, can be reduced in the shadow of a barrier which measures about 5 feet $(1.5 \mathrm{~m})$ on a side by at least $10 \mathrm{~dB}$. Low-pitched noise, however, might be reduced by only 4 or $5 \mathrm{~dB}$. Figure 3-15 illustrates the application of measures 1 , 2 , and 3 .

If intruding sound originates out-of-doors, you may be able to quiet the interior of your house by increasing the sound insulation of the exterior shell. Windows and doors are common leaks for both sound and heat. Properly fitted and sealed storm windows and doors will cut down the 


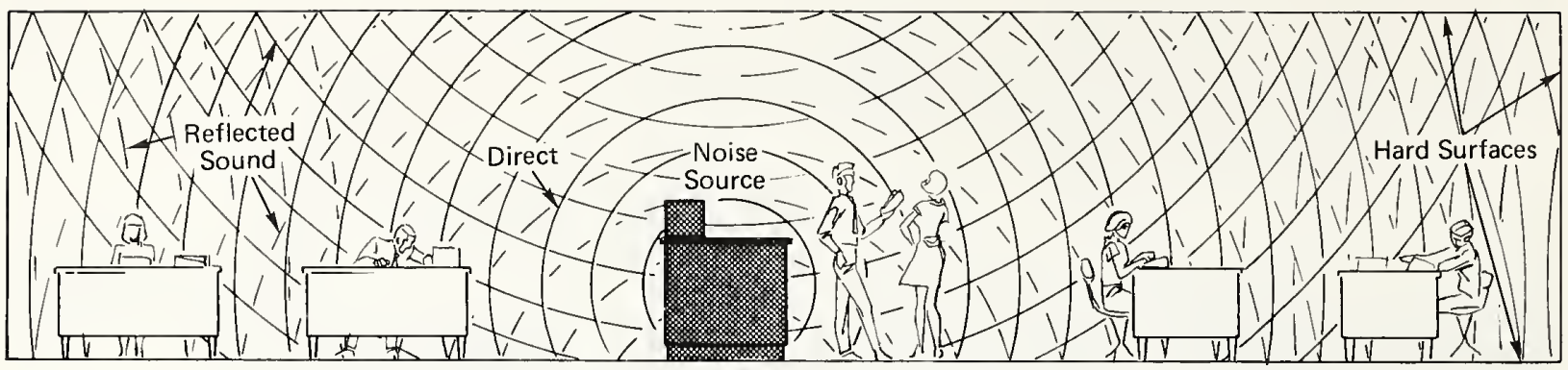

THIS

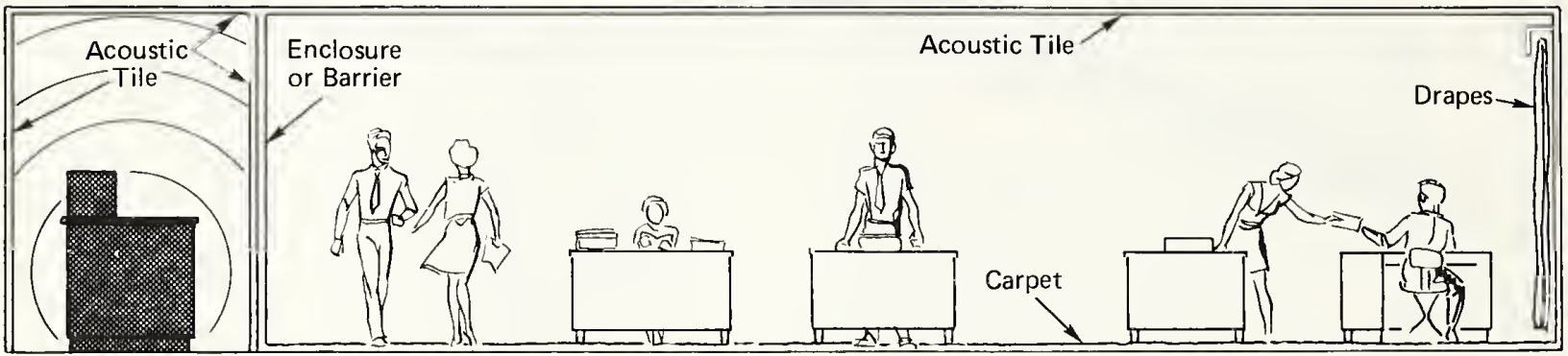

Fig. 3-15. NOISE CONTROL OF THE TRANSMISSION PATH

intrusion of outdoor sounds as well as reduce heat transfer.

\section{Use Acoustical Lining}

Noise transmitted through ducts, pipe chases or electrical channels can be reduced effectively by lining the inside surfaces of such passageways with sound absorbing materials. In typical duct installations, noise reductions of the order of 2 to $3 \mathrm{~dB}$ per linear foot of $1^{\prime \prime}(2.5 \mathrm{~cm})$ thick acoustical lining are quite possible for high-pitched sounds. (In metric units, the reduction is about $10 \mathrm{~dB} / \mathrm{m}$ for an acoustical lining $2.5 \mathrm{~cm}$ thick.) A comparable degree of noise reduction for the lower frequency sounds is considerably more difficult to achieve because it usually requires at least a doubling of the thickness and/or length of acoustical treatment. Various types of duct lining and silencers are illustrated in Fig. 3-16. 

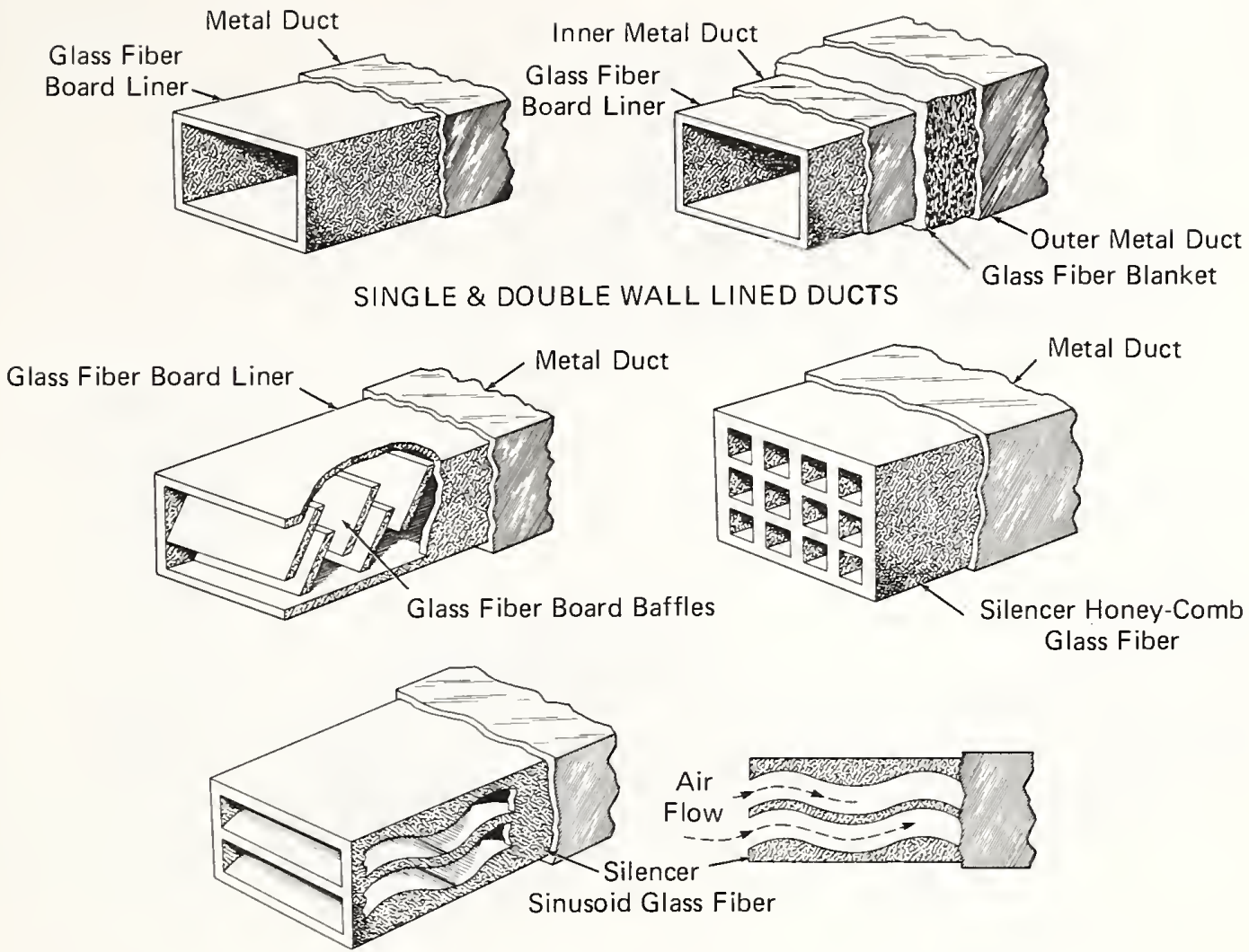

Fig. 3-16. VARIOUS TYPES OF ACOUSTICAL DUCT LINING, BAFFLES AND SILENCERS

5. Use Mufflers, Silencers, or Snubbers

Mufflers or silencers should be installed on all gasoline or diesel engines, regardless of size, including those used in model airplanes, toys, power tools, etc. Such devices should also be used in all installations in which large quantities of high-pressure, high-velocity gasses, liquids, steam or air are discharged into the open air as illustrated in Fig. 3-17. 


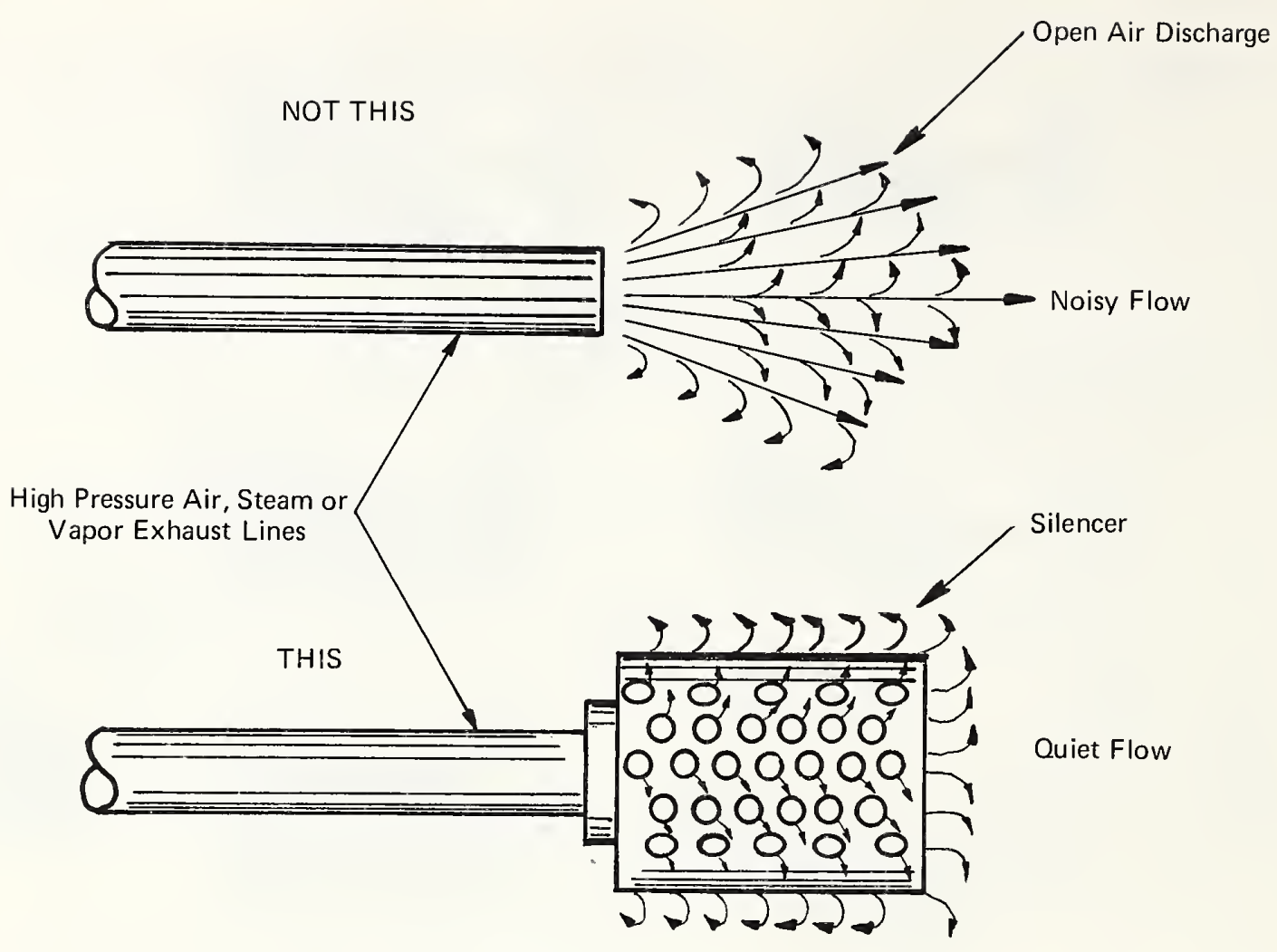

Fig. 3-17. SILENCER FOR HIGH PRESSURE EXHAUST LINE

6. Use Vibration Isolators and Flexible Couplers

In cases where the noise transmission path is structure-borne in character, vibration isolators in the form of resilient mountings, flexible couplers, or structural breaks or discontinuities should be interposed between the noise source and receiver. For example, spring mounts placed under an appliance or machine may prevent the floor from vibrating; or an expansion joint cut along the outer edges of a floor in a mechanical equipment room may reduce the amount of vibration transmitted to the structural frame or walls of a building. Such measures are illustrated in Figs. 3-18 and $3-19$. 


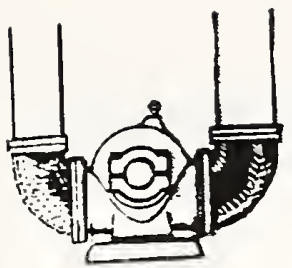

Pump Connector
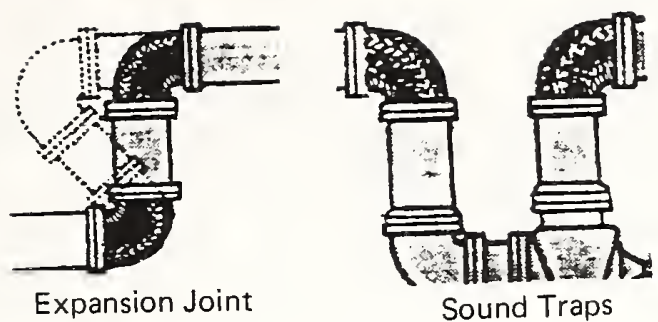
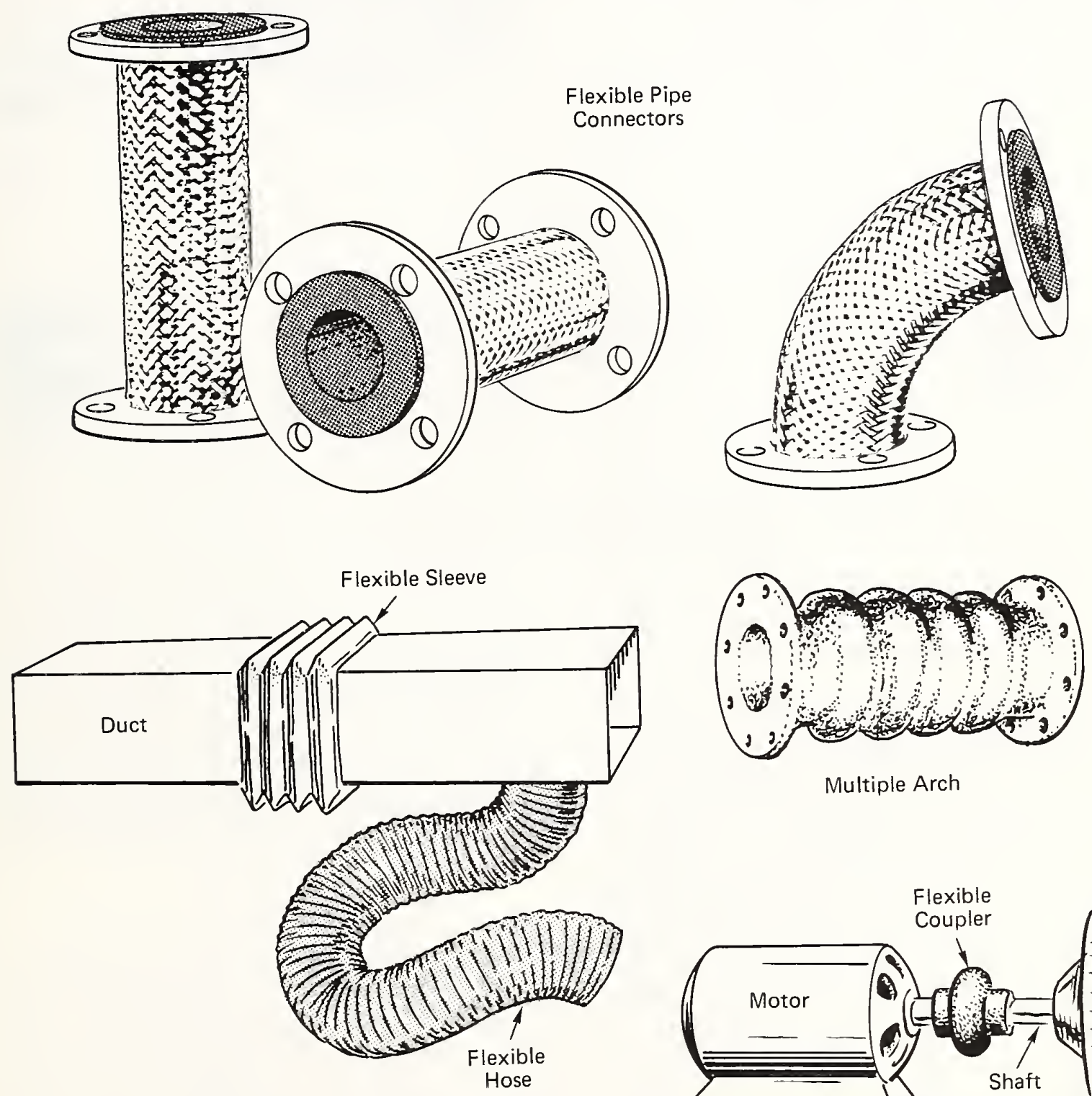

Multiple Arch

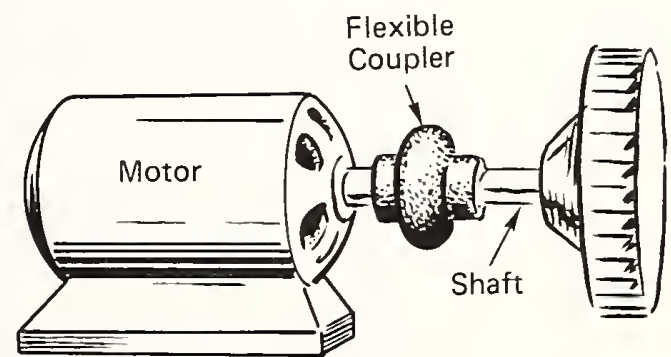

Fig. 3-18. VARIOUS TYPES OF FLEXIBLE CONNECTORS 

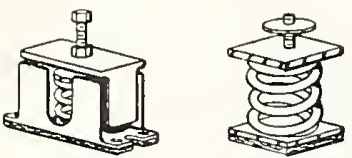

Spring Mounts

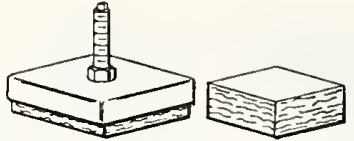

Fiberglass Mounts and Pads

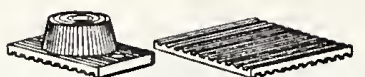

Neoprene Mounts and Pads
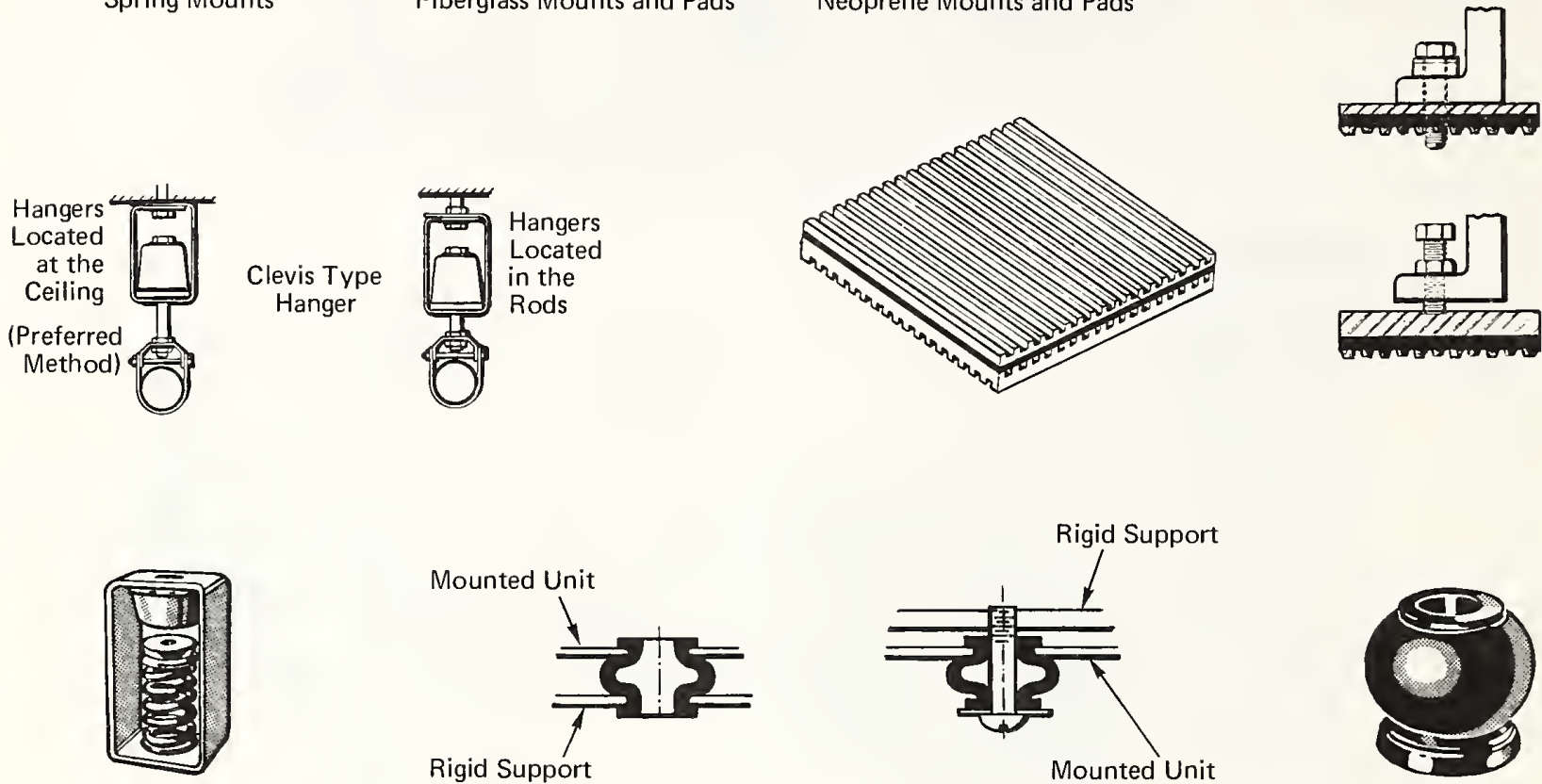

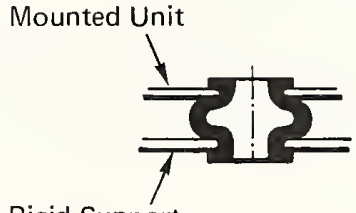

Rigid Support
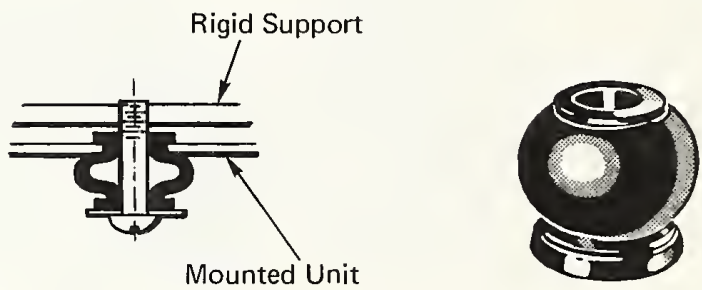

Fig. 3-19. VARIOUS TYPES OF VIBRATION ISOLATORS

\section{Use Enclosures}

Sometimes it is much more practical and economical to enclose a noisy machine in a separate room or box than to quiet it by altering its design, operation or component parts. The walls of the enclosure should be reasonably massive, and airtight to contain the sound. Absorbent lining on the interior surfaces of the enclosure will reduce the reverberant buildup of noise within it. Structural contact between the noise source and the enclosure must be avoided, or else the source vibration will be transmitted to the enclosure walls and thus short circuit the isolation. Figures 3-20 and 3-21 show the design and effectiveness of various enclosure configurations. Total enclosures should be used where large noise reductions are required, i.e., exceeding $15 \mathrm{~dB}$. Partial enclosures of $\mathrm{L}-$ or $\mathrm{U}$-shaped configurations may be adequate in areas requiring smaller noise reductions.

\section{PROTECT THE RECEIVER}

When exposure to iritense noise fields is required, as when operating chain saws or other very noisy equipment, measures must be taken to protect the receiver from hearing damage. This can be done by employing some of the following techniques.

\section{USE EAR PROTECTORS}

Molded and pliable earplugs, cup type protectors, and helmets are commercially available as hearing protectors. Such devices may provide noise reductions ranging from 15 to $35 \mathrm{~dB}$. However, such devices should be used only as a last resort, after all other methods have failed to lower the noise level to acceptable limits.

\section{ALTER. WORK SCHEDULE}

Suppose you have done all that it is possible to do to quiet an appliance - and 
still it makes noise! You should then operate the unit at times which will cause the least amount of disturbance to you and especially your neighbor. Waiting until later on Saturday and Sunday mornings to operate your lawn mower and power saw won't reduce their noise levels, but it may reduce the intensity of your neighbor's complaint. Inherent1y noisy operations, such as street repair, municipal trash collection, factory operation and aircraft traffic should be curtailed at night and early morning to avoid disturbing the sleep of the community.

Limit the amount of continuous exposure to high noise levels. In terms of hearing protection, it is preferable to schedule an intensely noisy operation for a short interval of time each day over a period of several days rather than a continuous 8-hour run for a day or two.

At home there are several intensely noisy chores such as gardening or landscaping with power mowers, tillers, and chain saws to name a few. If the home owner is concerned about protecting his hearing, he should intersperse periods of quiet activity during such chores to rest his ears. In short, he should avoid finishing intensely noisy jobs in continuous or overly prolonged runs, unless, of course, he is wearing ear protectors.

In an industrial plant employing a large number of people, an intermittent work schedule would benefit not only the operator of the noisy equipment but also other workers in the vicinity. Indeed, this practice would be even more beneficial if the noisy work were performed at night or at some other time when a minimum number of employees would be exposed. This assumes, of course, that noise created at night would be confined to the plant area and thus not disturb residential areas.

\section{IS THERE AN ACOUSTICAL PERFUME?}

Masking is a very prominent feature of the behavior of our ears. If you listen to a pure tone against a background of riva1 sounds, it will seem to fade as the rival background increases. In fact, the sounds of ordinary surroundings are sufficient to obscure recognition of our heart beats and breathing, which are quite audible in a very quiet place. For some people, the awareness of breath sounds is enough to break up the rhythm of breathing, and this becomes a source of discomfort.
However, sounds are masked only by rival sounds that are quite near them in pitch. Thus, the songs of birds cannot obscure the snoring of a person sleeping. Although lower-pitch masking has a greater effect on high-pitch sounds, the requirement that the masker must be in the frequency range of the tone to be masked is dominant, as we can recognize from the fact that orchestral music is heard as an ensemble. Effective masking requires a broad-band source of masking sound, if you can't predict the frequency range of the noise, or have to deal with broad-band interference.

In order to make conversational speech less obtrusive, as in a crowded restaurant, some use has been made of background music as an "acoustical perfume". Clearly the requirement for broad-band masking imposes the use of relatively bland, complex harmony, with a limited range of volume. Other usable sources of acoustical perfume are rippling brooks and splashing fountains.

However, efforts to use acoustic perfume for reduction of noise annoyance are not likely to succeed if the noise levels rival the levels of conversational speech. In order to override these levels, the background sound must begin to intrude into the higher levels where the ear's behavior is nonlinear. As a result, sounds would no longer necessarily retain their harmonious character. Where people working in noisy areas have been allowed to bring in radios, it will be observed that the listeners prefer strong rhythmic patterns and solo melocies.

A general characteristic of acoustical perfume is evident: it can be used to enhance the surroundings in an excessively quiet environment. People prefer some noise as opposed to not enough noise or too much noise. Most people feel quite comfortable in an environment with a low leve1, steady, soothing, unobtrusive sort of background noise, such as can be found along secluded beaches, forests, or quiet countrysides. However, it is impossible to produce a peaceful environment in an already noisy place merely by obscuring the sounds with rival stimuli.

Although the use of masking noise can be beneficial in certain cases, it has been extended and overemphasized to the point where it fails more frequently than it succeeds. The concept of masking noise in this context simply involves using an artificialiy induced noise or the ambient acoustical environment beneíicially for 

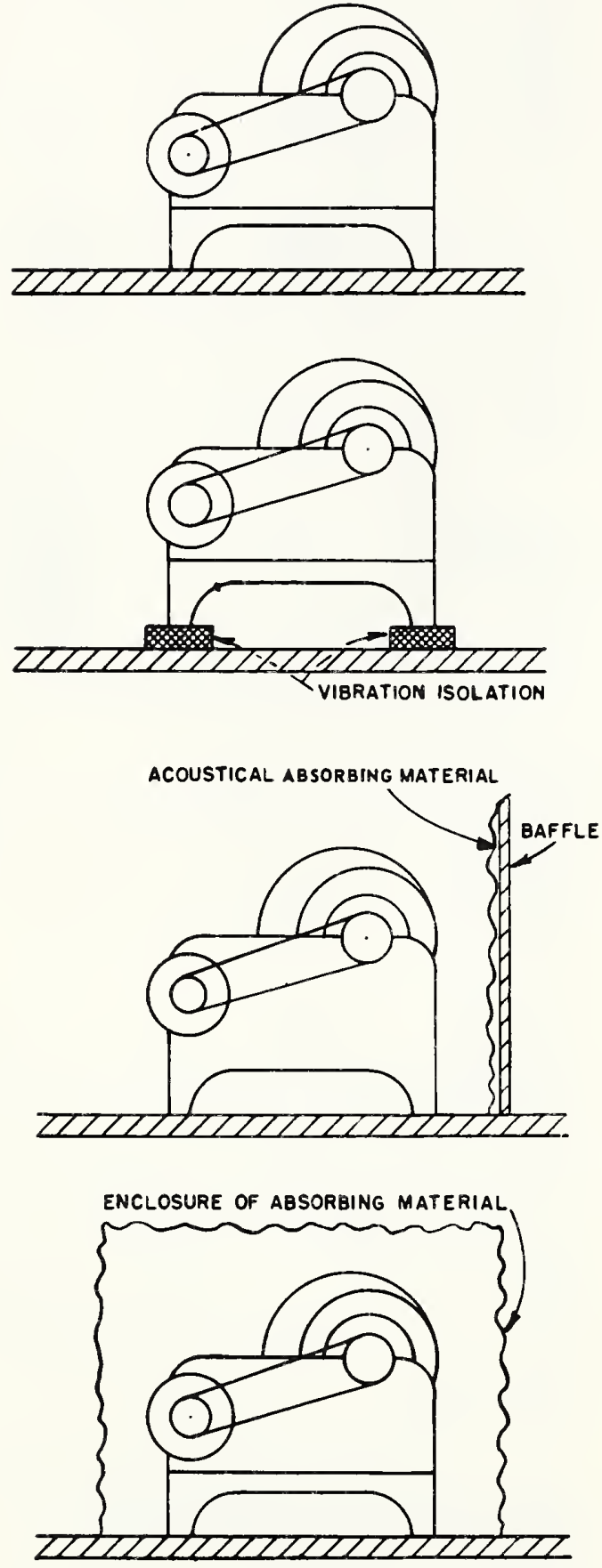

Courtesy of General Radio Company
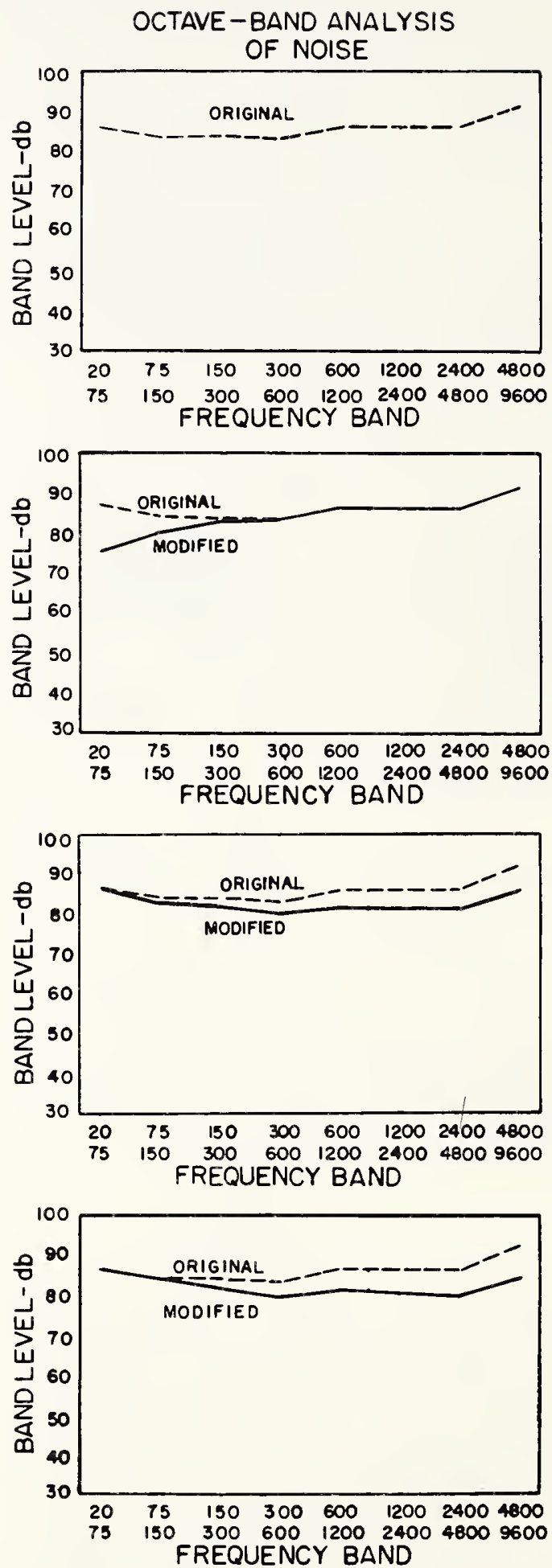

Fig. 3-20 EFFECTIVENESS OF VARIOUS NOISE REDUCTION TECHNIQUES 

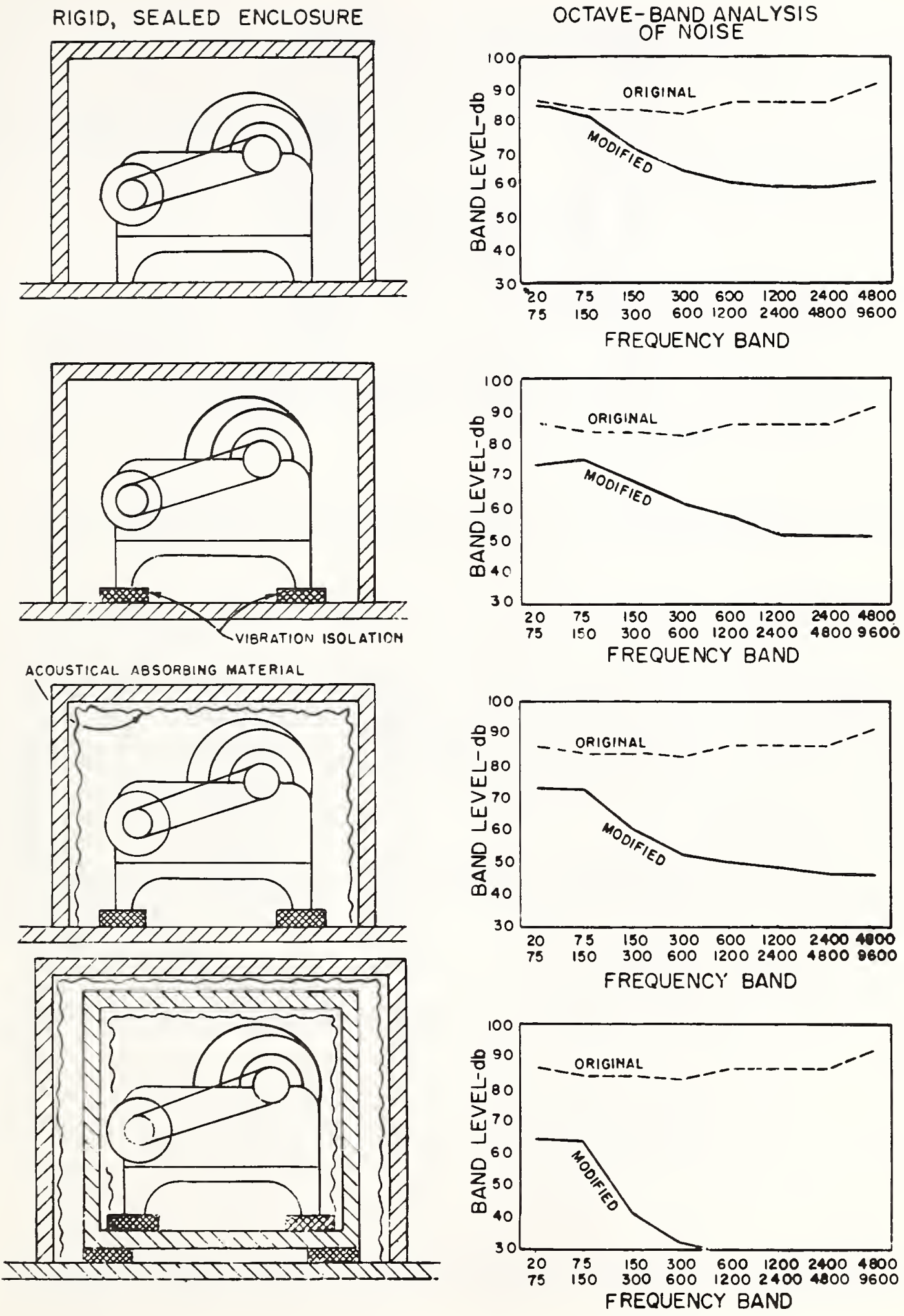

Fig. 3-20 EFFECTIVENESS OF VARIOUS NOISE REDUCTION TECHNIQUES 
Install Double Wall for

Optimum Sound Isolation

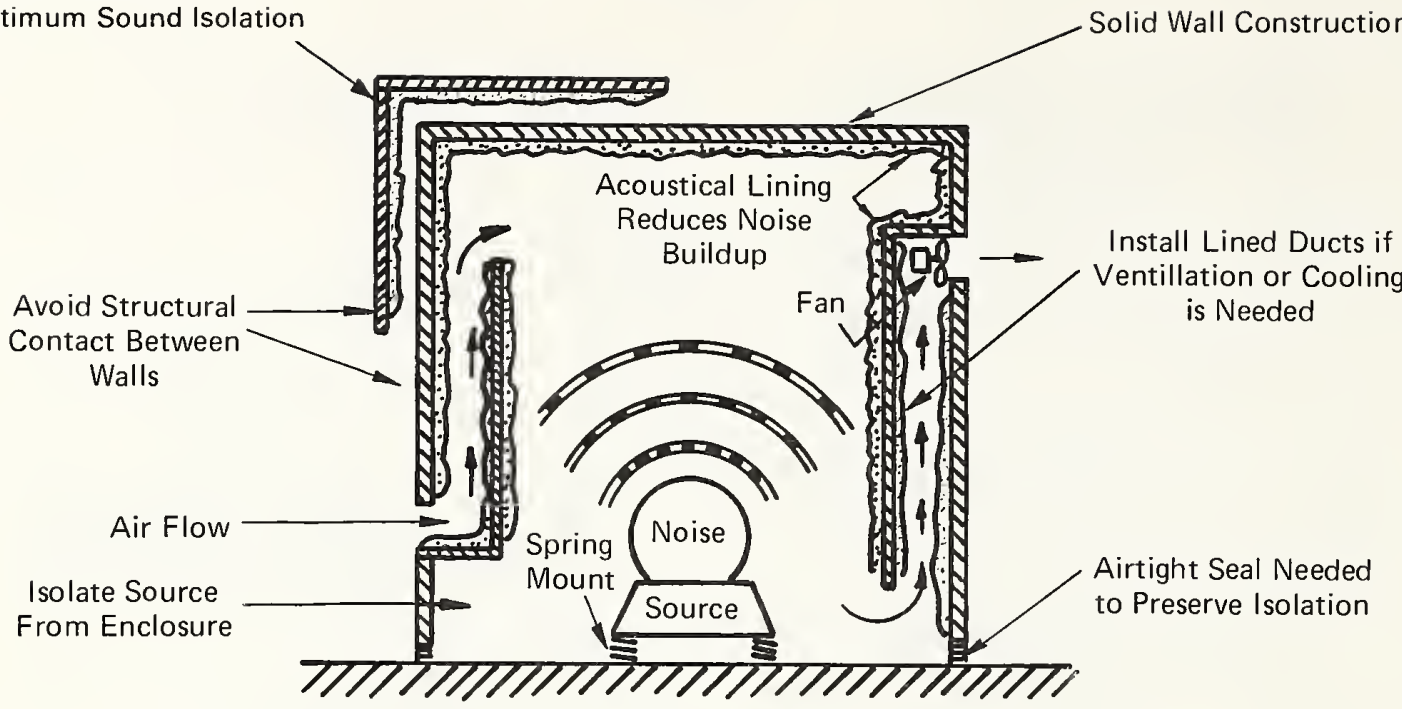

Fig. 3-21. DESIGN OF AN EFFECTIVE SOUND ISOLATING ENCLOSURE REQUIRING AIR CIRCULATION FOR COOLING

masking or overriding annoying intruding sounds. A descriptive definition of masking noise might be any steady sound which has the following pleasing characteristics:

(a) low intensity with a wide-band frequency distribution, void of any pure-tone components;

(b) an omnidirectional source, such that its location is not evident to the observer;

(c) the ability to over-ride or mask intruding noise without becoming annoying itself.

Many examples of sources of masking noise are given in the literature; the most commonly mentioned being vehicular traffic and heating and air conditioning equipment. Unfortunately neither is suitable. Traffic noise is seldom steady, and the heating or air- conditioning equipment is cyclic.
Hence such masking noise may itself become a source of annoyance. Likewise, using acoustic perfume to "trade off" or compensate for acoustically inferior party walls in the design of apartment buj.ldings usually backfires. However, commercial devices which electronically produce masking noise can be used to improve privacy in specialized situations. For example, using acoustic perfume in reception rooms or offices of dcctors, dentists, or lawyers provides additional privacy for the patient or client.

However, the presence of masking noise by whatever name interferes to some extent: with the ability to discriminate sounds. Thus, the presence of acoustic perfume in a doctor's office might interfere with his stethoscopic examination of a patient. Physicians have reported, for example, that it becomes difficult to interpret the sounds from a stethoscope if the surrounding sound levels exceed $45 \mathrm{~dB}$. 


\section{NOISE CONTROL AT HOME}

The peace and quiet traditionally associated with the home is fast disappearing. Indeed at times the noise levels within and outside the home or apartment approach those found in industrial plants. As a consequence, the typical homeowner or apartment dweller finds it rather difficult to isolate himself from the noise. This unfortunate situation is due primarily to the following factors:

(a) Increasing use of noisy appliances in virtually all areas of the home.

(b) The open-space layout of many homes. Wide doorless passageways between rooms, waist-high partial walls, and open screen room dividers permit noise to travel freely to all areas of the home.

(c) The poor sound insulation provided both by the exterior shell of the dwelling and by the partition walls, floors, and doors within. (d) The intrusion of neighborhood noise, the level of which unfortunately is increasing with the growth of automobile and aircraft traffic, and with the use of outdoor powered equipment and appliances.

Figures 4-1 and 4-2 illustrate some of the more common indoor and outdoor sources of noise that the typical apartment dweller or homeowner is exposed to on a daily basis.

TRANSMISSION OF AIRBORNE AND STRUCTUREBORNE NOISE:

The control of noise in the home involves reducing the travel or transmission of both airborne noise and structureborne noise, whether generated by sources within or outside the home. By airborne noise we mean noise that is produced initially by a source which radiates directly into the air. Many of the noises we encounter daily are of airborne origin; for example, the roar of an overhead

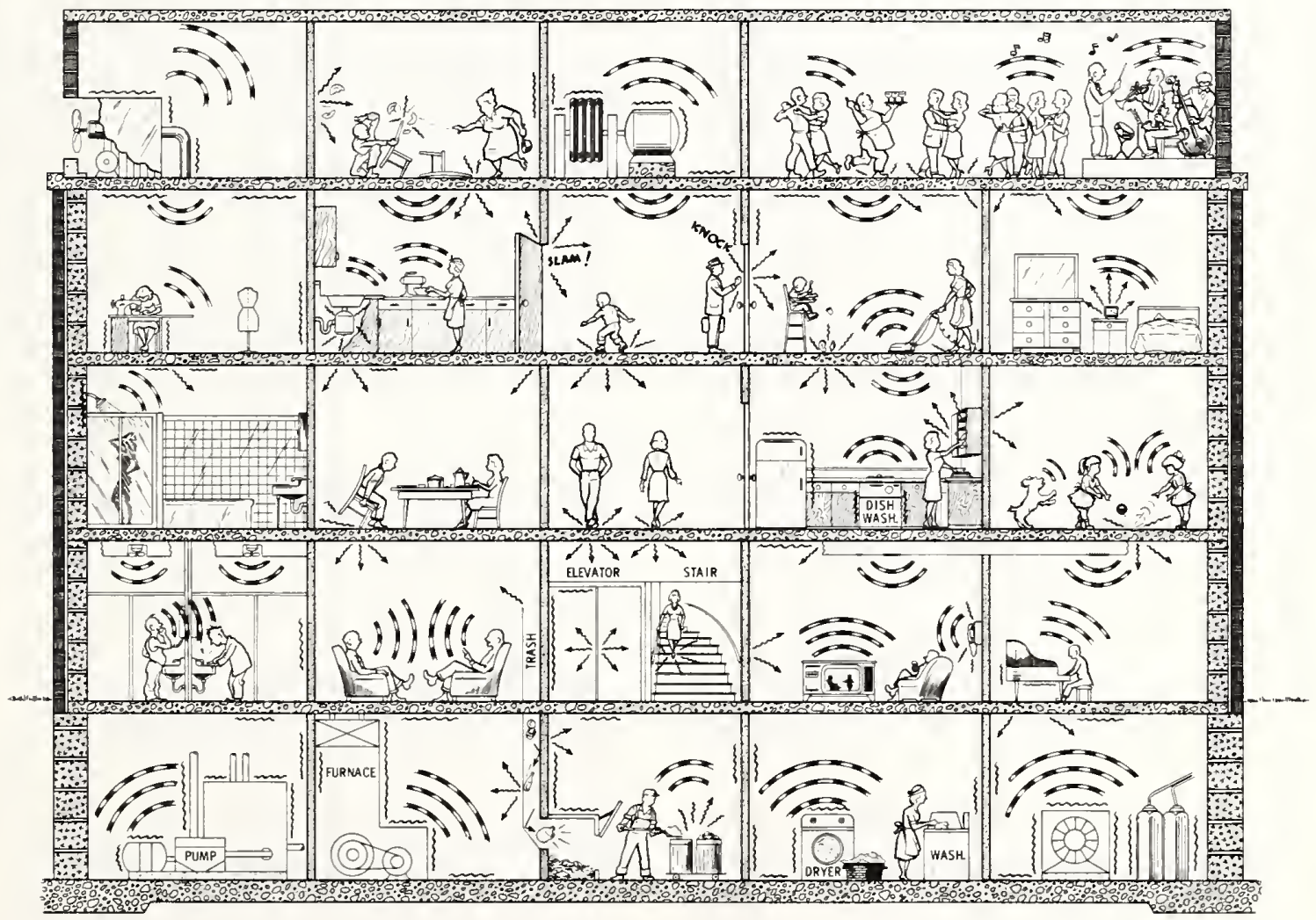

Fig. 4-1. COMMON INDOOR SOURCES OF NOISE 


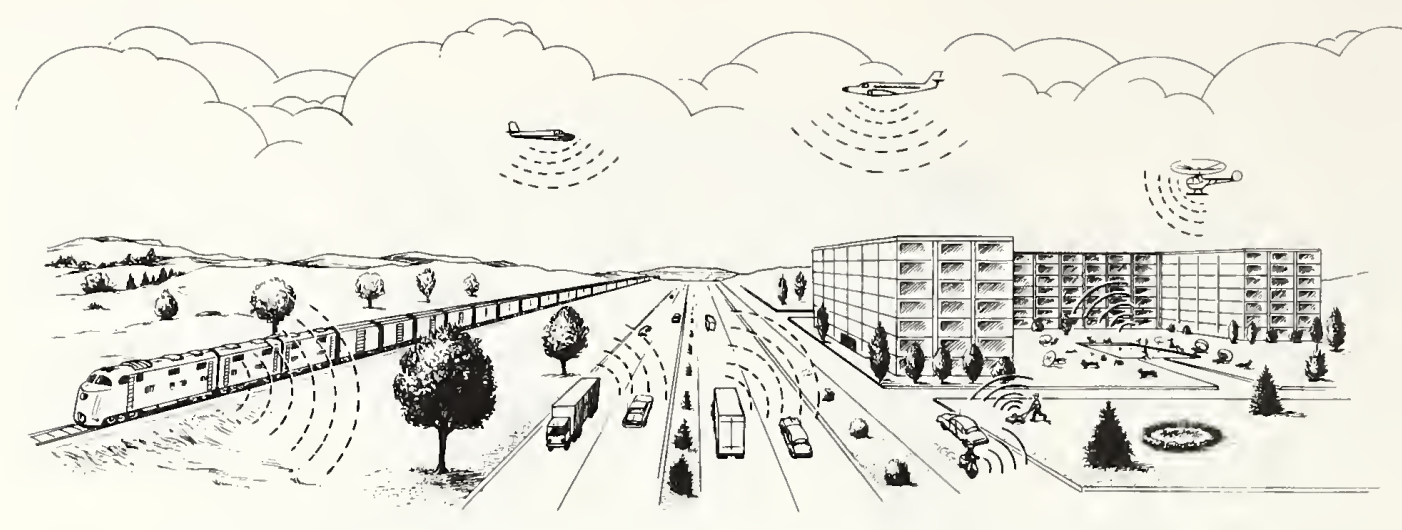

Fig. 4-2. COMMON OUTDOOR SOURCES OF NOISE

jet plane, the blare of an auto horn, voices of children, or music from stereo sets. Airborne sound waves are transmitted simply as pressure fluctuations in the open air, or in buildings along continuous air passages such as corridors, doorways, staircases and duct systems.

The disturbing influences of airborne noise generated within a building generally are limited to the areas near the noise sources. This is due to the fact that airborne noises usually are of much smaller power and are attenuated more easily than structureborne noise. The sound from your neighbor's stereo system may cause annoyance in rooms of your apartment which are adjacent to his, but rarely in rooms farther removed unless doors or passageways are open.

Structureborne noise occurs when wall, floor or other building elements are set into vibratory motion by direct contact with vibrating sources such as mechanical equipment or domestic appliances. This mechanical energy is transmitted throughout the building structure to other wall and floor assemblies with large surface areas, which in turn are forced into vibration. These vibrating surfaces, which behave somewhat like the sounding board of a piano, transmit their motion to the surrounding air, causing pressure fluctuations that are propagated as airborne noise into adjacent areas.

The intensity of structureborne noise generally is much higher than that produced by a wall or floor structure when it has been excited into vibration by an airborne sound wave because the vibrating source is more efficiently coupled to a structural member than it is to the surrounding air. Unlike sound propagated in air, the vibrations are transmitted rapidly with very little attenuation through the skeletal frame of the building or other structural paths. These vibrations frequently reach large light-frame structures which perform rather efficiently as sounding boards, and radiate the noise at high levels.

\section{"SOUNDING BOARD" EFFECT}

It might be well to consider briefly the so-called "sounding board effect", a reinforcement or amplification of sound, which so frequently is involved in the radiation of structureborne noise. Generally speaking, the efficiency of a sound radiator varies directly with the ratio of its surface area to the wavelength of sound; the larger the area of a radiator the greater its effectiveness. A sound source with a small radiating surface, such as a water pipe, produces relatively little airborne sound; but on the other hand, it will radiate high-pitched sounds more efficiently than low-pitched sounds, all other factors being equal. If a small vibrating source, which by itself radiates little airborne noise, is rigidly or mechanically coupled to a large surface such as plywood or gypsum wall panel, the intensity or volume of sound will be substantially reinforced or amplified. A piano provides an illustration of this effect. If we were to remove the sounding board of a piano, the sound generated by the vibrating strings would be almost inaudible, because of their small radiating surface.

Under certain conditions, airborne noise may produce structureborne noise which in turn may be radiated again as airborne 
noise. For example, an airborne sound wave striking one side of a wall may cause the entire wall to vibrate like a solid panel. Thus, the sound is transmitted to the other side of the wall from which it is radiated as airborne sound. However, some of the wall vibration will be transmitted to the supporting floor, adjacent walls, and through other structural members to various parts of the building where it eventually emerges as airborne sound.

There are many noise sources which generate both airborne and structureborne noise simultaneously. Sources usually considered to be strictly airborne noise generators may produce a substantial amount of structureborne noise and vibration if they are fastened rigidly to wall and floor structures. For example, a loudspeaker built into a wall enclosure might cause not only the wall to vibrate but perhaps the rafters as well. Every noise source has vibrating elements which radiate noise. A window air conditioner suspended in mid air would produce a substantial amount of airborne noise; however, when the unit is mounted in a conventional manner a combination of both structureborne and airborne noise of greater intensity is produced, due to the induced vibration of the window and supporting wall. Occasionally, a noise source may produce vibrations so low in frequency that they can be felt but not heard. In some instances, such a source may induce a wall or floor structure to resonate at its own natural frequency, which may be in the audible range. Thus, the low-pitched drone of a passing airplane may cause a wall or window to resonate at a higher pitch than that radiated by the plane itself.

\section{FLANKING NOISE TRANSMISSION}

The movement of noise from one completely enclosed room to an adjoining room separated by a continuous partition wall may be either by direct transmission through that wall, or by indirect transmission through adjoining walls, ceilings and floors common to both rooms or through corridors adjacent to the room. Noise transmission by indirect paths is known as flanking transmission. Quite frequently one is faced with a noise problem that involves a combination of both direct and indirect transmission paths, and in which noise from the indirect path may be the more serious offender. Such indirect or flanking transmission commonly occurs with structureborne as well as airborne noise.
The chief flanking transmission paths of airborne noise between two adjacent rooms usually involve common corridors, ventilation grilles, duct systems, open ceiling plenums which span both rooms, louvered doors, and close spacing of windows between rooms. In addition to the flanking paths, there may be noise leaks, particularly along the ceiling, floor and side edges of the partition wall. Also, noise leaks may occur around pipe and conduit penetrations, back-to-back installations of cabinets and electrical outlets in the partition wall. Imperfect workmanship may result in serious noise leaks, e.g., poor mortar joints in masonry core-walls which often are concealed behind furred walls, panels or built-in cabinets.

As we see in Fig. 4-3 airborne noise may travel from one room to another over a number of different paths, some of which are not only complicated but often hidden from view.

Flanking transmission paths of structureborne noise, as illustrated in Fig. 4-4, are about as numerous and difficult to trace as those of airborne noise. Noise and vibration-producing equipment such as fans, compressors, pumps, ventilation and plumbing systems readily communicate their vibrational energy to the building structure if no precautionary measures are taken. The vibration travels quickly over long distances through the skeletal building structure with no appreciable attenuation, forcing other building elements like wall and floor assemblies in other parts of the building to vibrate and to radiate noise. While noise or vibration transmission problems between adjacent rooms are relatively simple to resolve, it may be extremely difficult to determine the reasons for excessively high noise or vibration levels in rooms far removed from the noise sources. The difficulty arises in tracing the specific flanking paths and identifying the operating equipment at fault.

A good rule to follow is to conduct an inspection of the apartment building in order to locate a 11 excessively noisy areas. Identify and examine the major noise sources in each area, including their mountings and other accessory equipment and hardware to which they are connected. Then, starting with the noisiest source try to reduce as much as possible the airborne noise radiation and vibrational motion of the source, using the various techniques discussed throughout this guide. This, of course, presupposes that the building owner 


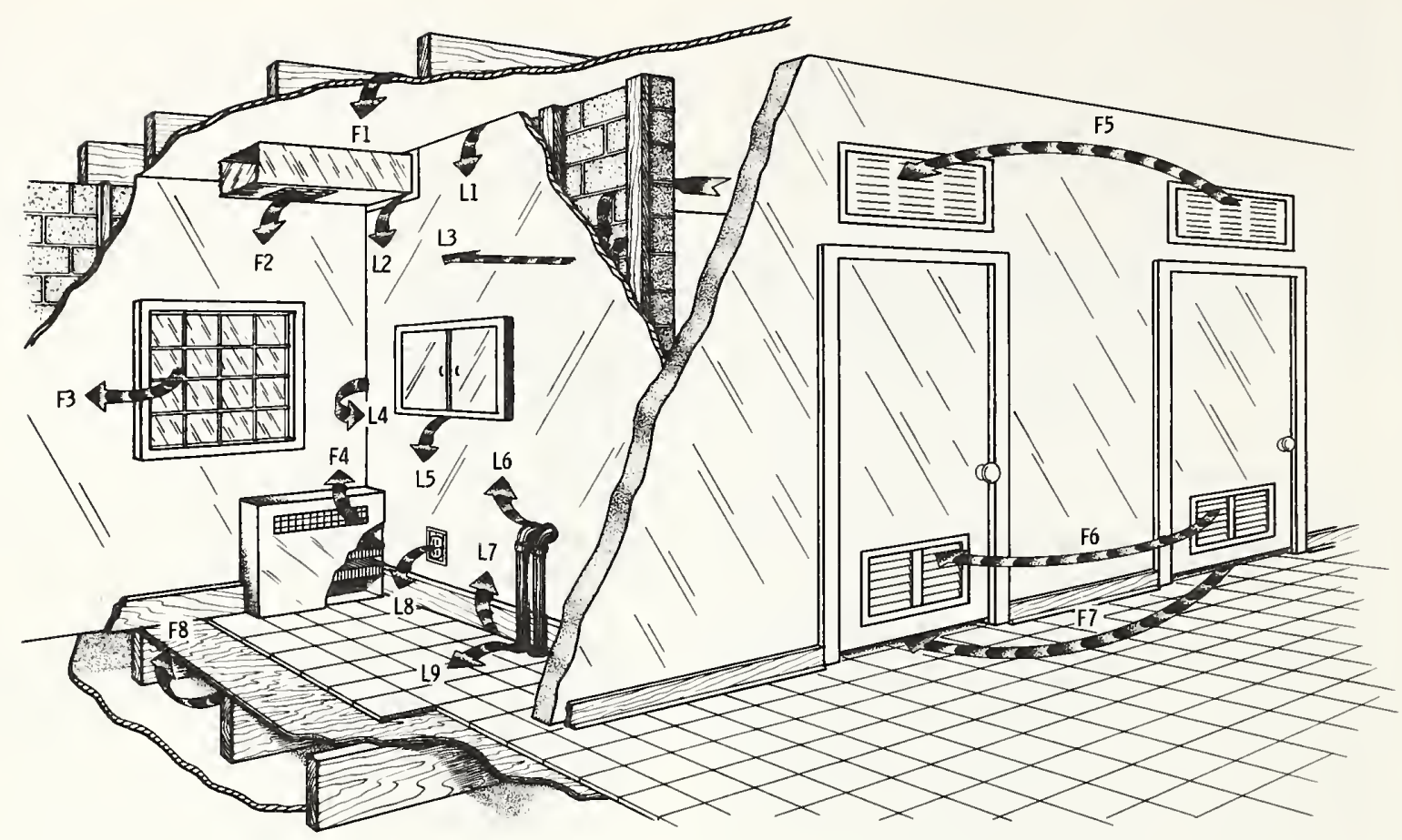

FLANKING NOISE PATHS

FI OPEN PLENUMS OVER WALLS, FALSE CEILINGS

F2 UNBAFFLED DUCT RUNS

F3 OUTDOOR PATH, WINDOW TO WINDOW

F4 CONTINUOUS UNBAFFLED INDUCTOR UNITS

F5 HALL PATH, OPEN VENTS

F6 HALL PATH, LOUVERED DOORS

F7 HALL PATH, OPENINGS UNDER DOORS

F8 OPEN TROUGHS IN FLOOR-CEILING STRUCTURE
NOISE LEAKS

LI POOR SEAL AT CEILING EDGES

L2 POOR SEAL AROUND DUCT PENETRATE

13 POOR MORTAR JOINTS, POROUS MASONRY BLK

L4 POOR SEAL AT SIDEWALL, FILLER PANEL ETC.

15 BACK TO BACK CABINETS, POOR WORKMANSHIP

L6 HOLES, GAPS AT WALL PENETRATIONS

L7 POOR SEAL AT FLOOR EDGES

L8 BACK TO BACK ELECTR ICAL OUTLETS

L9 HOLES, GAPS AT FLOOR PENETRATIONS

OTHER POINTS TO CONSIDER, RE: LEAKS ARE (A) BATTEN STRIP A/O POST CONNECTIONS OF PREFABRICATED WALLS, (B) UNDER FLOOR PIPE OR SERVICE CHASES, (E) RECESSED, SPANNING LICHT FIXTURES, (D) CEILING \& FLOOR COVER PLATES OF MOVABLE WALLS, (E) UNSUPPORTED A/O UNBACKED WALL BOARD JOINTS (F) EDGES \& BACKING OF BUILT-IN CABINETS \& APPLIANCES, (G) PREFABRICATED, HOLLOW METAL, EXTERIOR CURTAIN WALLS.

Fig. 4-3. COMMON AIRBORNE NOISE TRANSMISSION PATHS IN BUILDINGS

or management will permit such a survey to be done. However, most owners faced with noise complaints can be persuaded to cooperate, especially if the chances are good that the installation of some inexpensive treatment or simple devices would alleviate the noise problem, as for example inserting vibration isolators under a pump or elevator motor; or perhaps installing pressure reducing valves in certain locations of the building plumbing system.
GENERAL SOLUTIONS FOR NOISE CONTROL IN THE HOME

General solutions for the control of noise in the home or apartment involve a three step approach, namely:

(1) Stop or reduce the transmission of airborne sound by installing a wall or floor structure in its path. Ideally, the structure should reduce the level of 


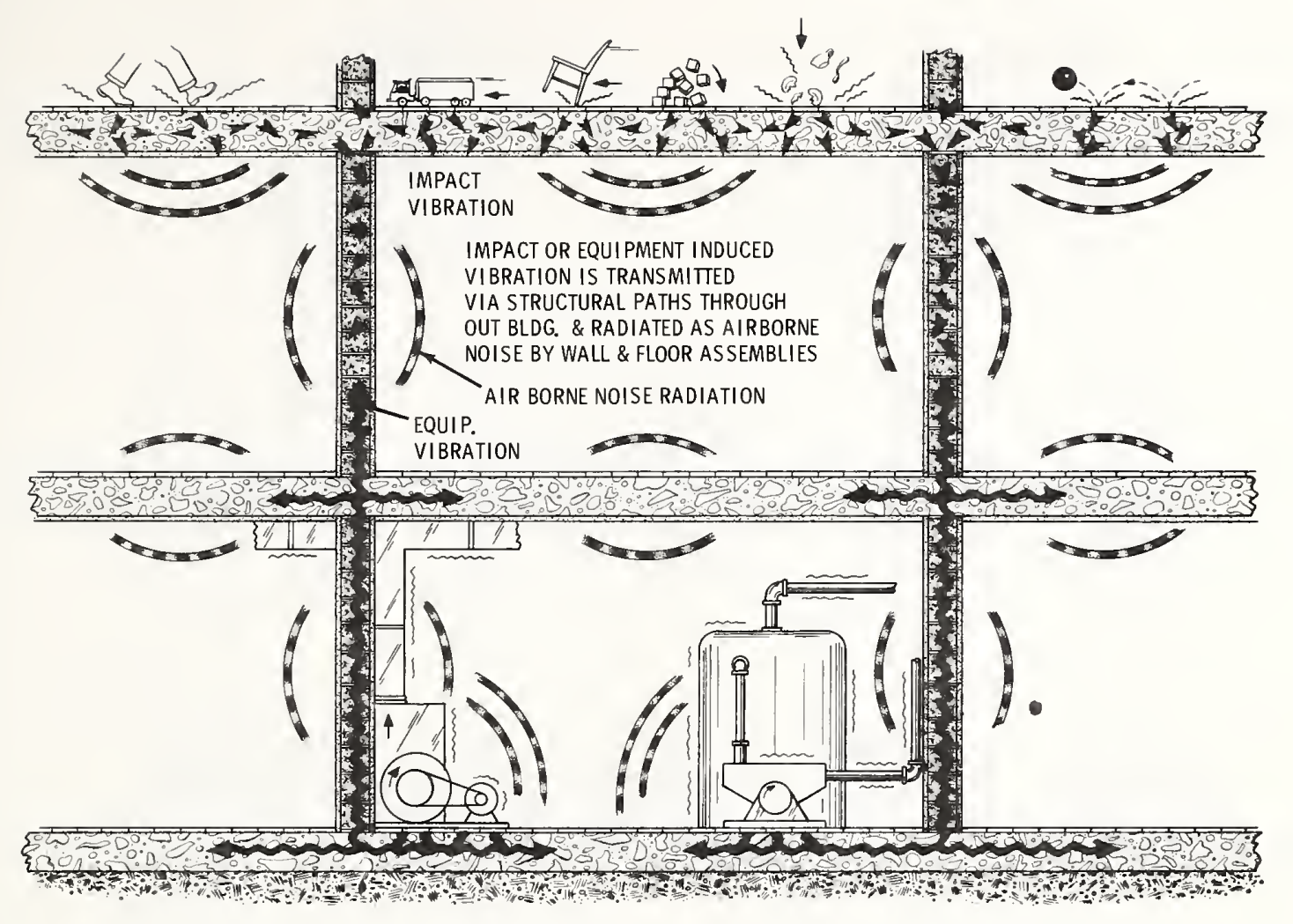

Fig. 4-4. COMMON STRUCTURE-BORNE NOISE AND VIBRATION PATHS IN BUILDINGS

intruding sound sufficiently to make it inaudible, or at least unnoticeable in areas where privacy is desired. The sound insulating effectiveness of a single wall is dependent on weight; there is a gain of about 5 $\mathrm{dB}$ in sound insulation or transmission loss for each doubling of the weight of the wall. However, single walls are not very effective where a high degree of sound insulation is required. For the same overall weight, a double wall or one using resilient studs or channels can be made to provide a higher degree of sound insulation than a single wa11.

(2) Stop or reduce the transmission of impact or structureborne noise by installing a structure, whether a wall or a floor, that will cushion the impact or interrupt the path of vibration. In the case of a concrete floor slab, a thick soft carpet with felt or rubber padding will be adequate. A floor of light-frame construction might require not only carpeting, but also a gypsum board ceiling suspended on resilient channels to achieve the same degree of impact isolation.

(3) Stop or reduce sound reflection which tends to amplify or buildup the level of noise, particularly in rooms. Ordinary room furnishings like carpeting, drapery and upholstered furniture are effective sound absorbers and should, therefore, be used to control excessive reflection. Sound reflection off hard ceiling surfaces can be minimized by the installation of an absorptive ceiling.

Techniques for the control of airborne and structureborne noise transmission through wall and floor structures are illustrated in Fig. 4-5. 

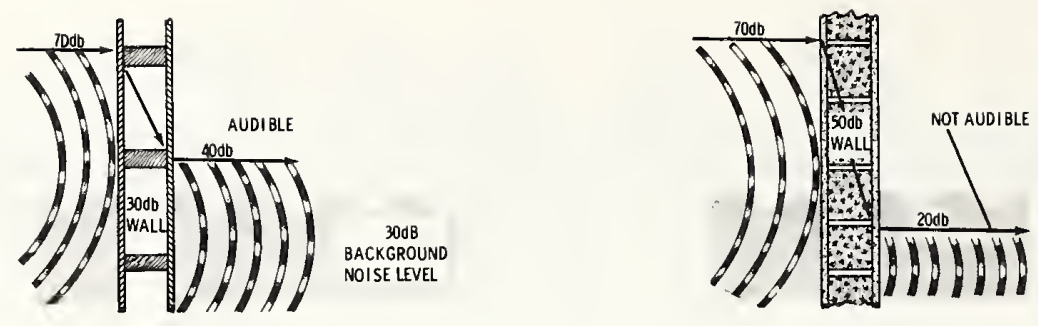

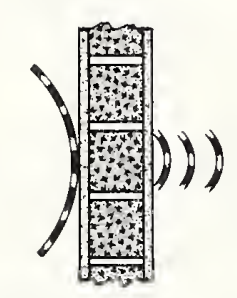

A. MASSIVE WALL

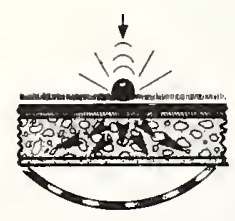

A. CUSHIONIMPACT

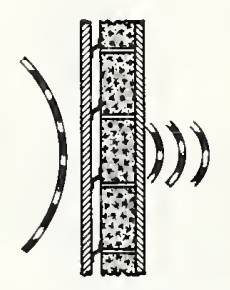

B. ISOLATED SURFACE

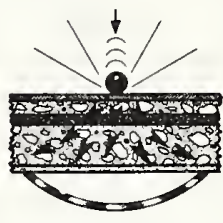

B. FLOAT FLOOR

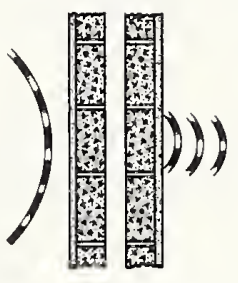

C. DOUBLE WALL

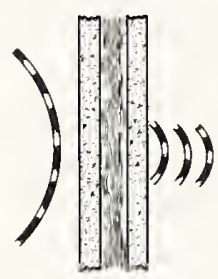

D. SOUND ABSORBER IN CAVITY

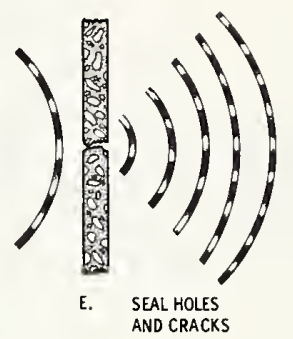

AND CRACKS

Fig. 4-5. METHODS OF CONTROLLING AIRBORNE AND IMPACT SOUND TRANSMISSION THROUGH WALLS AND FLOORS

\section{SOUND ABSORPTION VERSUS SOUND INSULATION}

The reduction and control of noise, whether in machinery, buildings, or rooms, generally involves the use of sound absorbing materials and sound insulating materials. These materials have different properties and are used for different purposes, which can not be interchanged. But due to confusion, both materials often are misused.

Sound absorbing materials such as acoustical tile, carpets and drapery play an indispensible part in controlling noise generated within a room or in reverberant areas such as lobbies, corridors and staircases. Although such materials are highly effective as sound absorbers, they are relatively poor sound insulators because of their soft, porous and lightweight construction. In short, they transmit noise very easily. To illustrate this point, imagine a wall constructed solely of acoustical tile, carpet or drapery material. Such a wall would provide virtually no resistance to the passage of sound through it. A brick wall on the other hand, is a very poor absorber of sound but it is an extremely effective sound insulator. Because it is massive, it resists the passage of sound. Perhaps a better example illustrating the difference between absorption and insulation is the ordinary blotter. The blotter is a very good absorber of liquids, but one would never consider using blotting paper to form the walls of a swimming pool. When the blotter absorbs the liquid to its saturation point, the liquid readily passes through it and leaks away.

Because sound insulators and sound absorbers are both called "acoustical treatment", confusion arises between their proper uses. Improper application of sound-absorbing treatment to reduce the transmission of noise between adjoining areas in an existing building has often been an expensive and annoying error.

WARNING: Sound-absorbing materials are 
generally light and porous. They should not be used on the interior surface of one room with the sole intention of preventing the transmission of sound to the next room. The porosity of the material actually facilitates sound transmission.

\section{KITCHEN NOISE}

With rare exception, the kitchen is the noisiest room in the home. This is due to its hard sound reflecting surfaces, and the frequent and oftentimes simultaneous operation of the various types of very noisy power-driven appliances found in the kitchen. These include garbage disposers, dishwashers, blenders, mixers, can openers, ice crushers, exhaust fans, etc. Although kitchen noise generally subsides before bedtime and thus rarely interfers with sleep, it ranks as one of the most irritating disturbances during daytime or early evening periods of relaxation and entertainment. Even though most kitchen appliances are operated for relatively short periods of time, it is the intensity, random intermittency and the periodic or cyclic characteristics of the noise that are especially annoying, as for instance the noise associated with filling, washing, draining and rinsing cycles of a dishwasher. Kitchen noise like most building noise problems can be avoided if good noise control techniques are incorporated early in the design stage of the home. This can best be done by adopting the following design and noise control features.

(1) Choose a floor plan which locates the kitchen as far as possible from such noise sensitive areas as bedrooms, studies or formal living rooms.

(2) Design the kitchen as a completely enclosed sound insulated room.

(3) Select "quiet" appliances and insist on proper vibration isolated mounting or installation, and

(4) Insta11 sound absorbing materials on room surfaces to reduce the noise buildup due to excessive reverberation. Materials such as acoustical ceiling tile, draperies and curtains, carpets or throw rugs and even racks of exposed towelling can provide a considerable reduction in kitchen noise level.

What can be done to control the noise from an existing kitchen with typical appliances and furnishings? A significant reduction of both the level of noise in the kitchen area and the amount of noise escaping to other areas of the home can be achieved by adopting the recommendations illustrated in Fig. 4-6, and described in greater detail below.

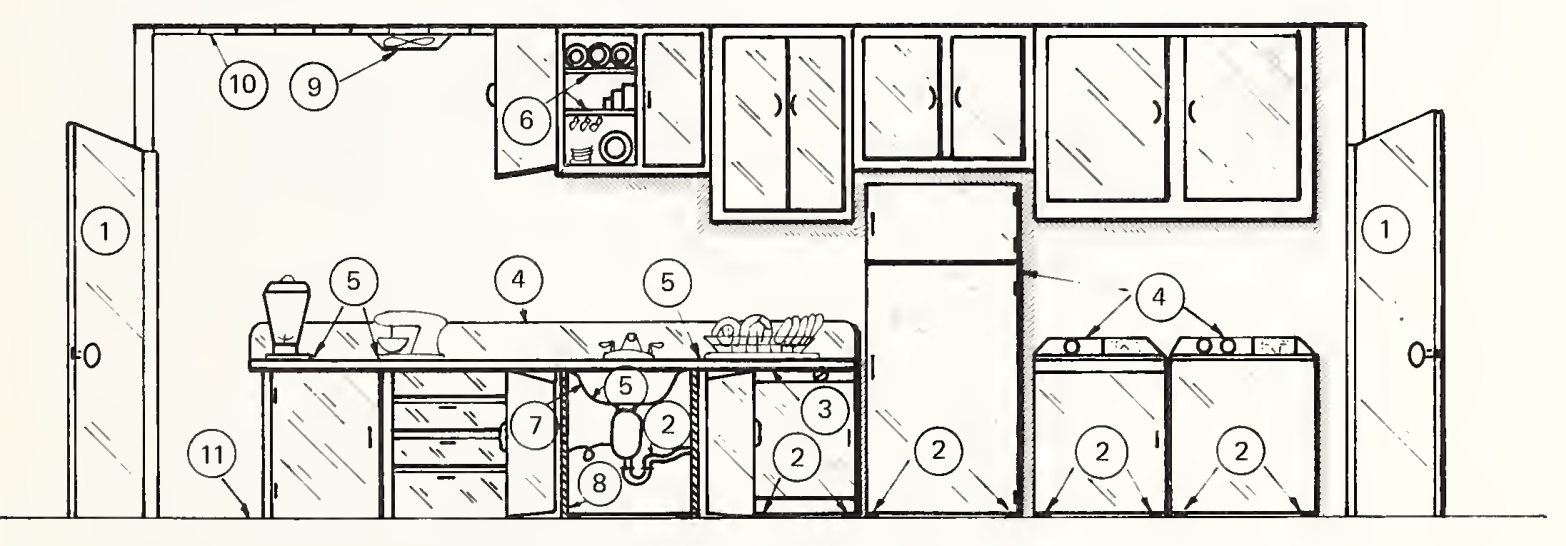

(1) Install solid door with gaskets

(2) Use vibration isolators \& mounts

(3) Isolate appliance from cabinet with rubber gasket

(4) Insert rubber gaskets behind cabinets and appliances to avoid wall contact

(5) Place rubber pads under small units, dish racks and in sink basins
(6) Install rubber or cork tile on backs and shelves of cabinets

(7) Apply vibration damping material

(8) Install acoustic tile

(9) Install exhaust fan on rubber mounts

(10) Install acoustic ceiling

(11) Install carpet or foam backed tile

Fig. 4-6. RECOMMENDATIONS FOR REDUCING KITCHEN NOISE 


\section{Instal1 Doors}

Completely enclose the kitchen area by installing doors in all passageways. Doors should be of solid core construction and equipped with soft rubber or plastic gaskets at the top, sides and at the bottom, as illustrated in Fig. 4-7. Hinged or sliding doors may be used, providing good edge seals are maintained. Such an installation will confine the noise to the kitchen area.

\section{Control Appliance Noise}

1. Vibration isolate the appliance from the floor

Place pads of resilient materials such as sponge rubber, ribbed neoprene or solid rubber under the legs or corners of large, heavy appliances like wash machines, dryers, and refrigerators to

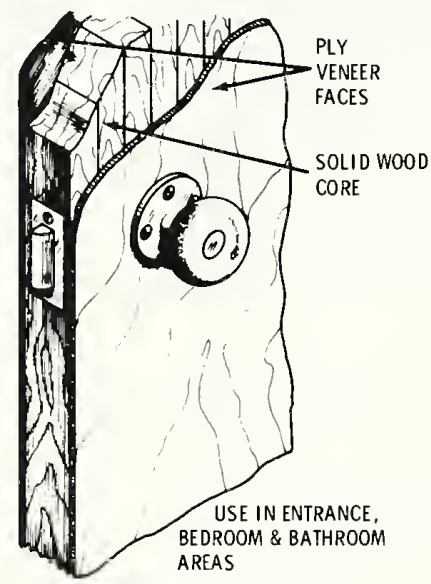

SOLID CORE DOOR prevent the transfer of machine vibration to the supporting floor. Pads measuring $1 / 4$ to $1 / 2$ inch ( 6 to $13 \mathrm{~mm}$ ) in thickness with an area of about 2 square inches ( 13 $\mathrm{cm}^{2}$ ) should provide adequate vibration isolation for most large appliances. To prevent screw-type legs from sinking too deeply in the pad, place a hard plate on top of the pad to distribute the load more uniformly. The legs may require some adjustment to prevent rattling against the hard plates.

\section{Isolate appliance from the wall}

Under no conditions should any appliance make rigid contact with a wall. A space of at least 2 inches $(5 \mathrm{~cm})$ should separate the appliance from the wall. Flexible plastic or rubber hoses should be

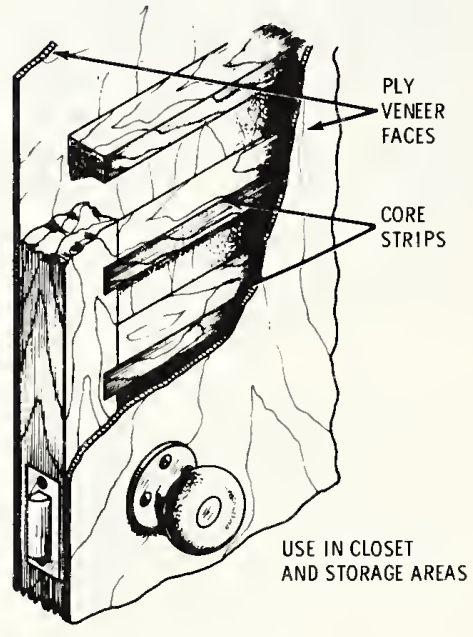

HOLLOW CORE DOOR
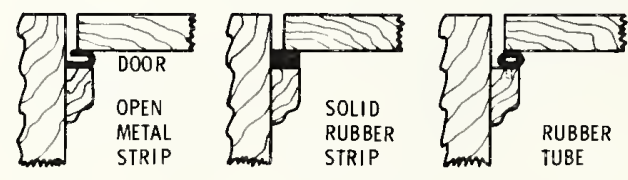

DOOR JAMB GASKETS

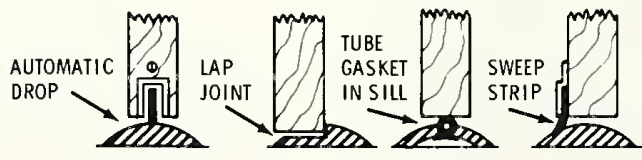

DOOR SILL CLOSURES

Fig. 4-7. PROPER INSTALLATION OF SOUND INSULATING DOORS 
used in the installation of food waste disposers, and of dish or clothes washers to avoid the transfer of machine vibration to the walls containing the plumbing outlets or fixtures. Otherwise, the vibrating walls acting like sounding boards would tend to amplify the machine noise.

3. Isolate the appliance from cabinet enclosure

Appliances such as dishwashers, disposers, compactors, etc., which are built into or enclosed by counter-type wall cabinets, should preferably be installed with a perimeter strip type gasket or spacers made of soft rubber to vibrationally isolate the cabinets from the machine. A strip gasket $1 / 4$ inch $(6 \mathrm{~mm})$ thick and $1 / 2$ inch $(13 \mathrm{~mm})$ wide attached to the top and side edges of the cabinet opening into which the appliance is to be installed would provide adequate isolation.

Small countertop appliances such as blenders, mixers, ice crushers, can openers, eic. should be placed on small pads of soft rubber to prevent the transfer of vibration to the countertop.

As a temporary measure, folded towels placed under small appliances can be effective. However, in using these schemes, one must be careful to avoid blocking any air vents used for cooling purposes that might be located in the base of the units. Otherwise, the appliances might tend to overheat with prolonged use.

A soft rubber sleeve-type gasket inserted between the mounting flange of a waste disposer and a sink basin will tend to prevent the sink from vibrating and thus amplifying the disposer noise. Figure 4-8 illustrates the proper installation of a disposer for quiet operation.

4. Isolate the cabinet enclosure from wa11

Countertop wall cabinets with built-in appliances tend to vibrate excessively when the devices are used. To prevent the transmission of the vibration to the back wall, strip gaskets, as described above, should be inserted behind the cabinet to avoid rigid contact with the wall. This will eliminate the tendency of the wall to act as a sounding board. Such gaskets will also reduce impact noise radiation caused by the stocking of cabinet shelves and the closing of drawers and cabinet doors.

\section{Isolate small appliances}

In most cases it is extremely difficult to reduce the noise output of the small countertop appliances that you presently own; the devices usually are built so compactly that it is virtually impossible to vibration isolate the case or housing from the motor and gear trains, which generally are the main sources of noise. The lack of room also precludes lining the case with sound

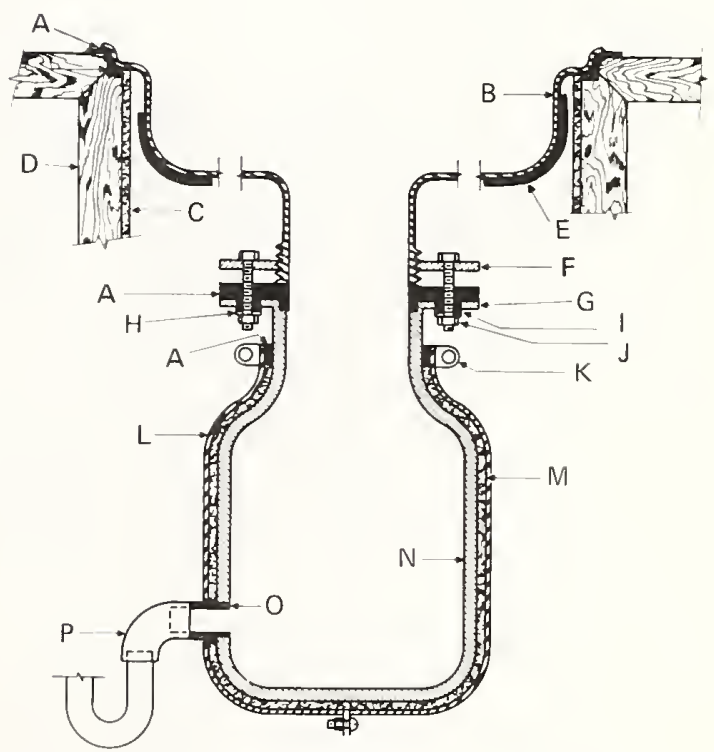

A - Rubber Gasket F-Ring Plate

$B-$ Sink

C - Acoustic Mat'|

$G$ - Disp'l-Flange

D - Cabinet

E - Mastic Coat

$\mathrm{H}$ - Steel Washer

1 - Rubber Washer

K - Ring Clamp

$\mathrm{L}$ - Metal Cover

$M$ - Glasswool

N- Disp'l-Housing

O - Rubber Sleeve

$\mathrm{P}$ - Rubber Hose

Fig. 4.8. DESIGN AND INSTALLATION OF A GARBAGE DISPOSER FOR QUIET OPERATION 
absorbent material. Sometimes lining the inside surfaces of an appliance with a metal or lead foil-backed adhesive-coated tape will damp the vibration of the case and thus result in some reduction of the noise. Aside from operating the units at low speeds, about all the owner can do is to vibrationally isolate the units from the counter tops by placing soft rubber pads under the base. This scheme at least prevents the counter top from amplifying the noise as a sounding board and thus results in a noticeable reduction in the overall noise.

Placing rubber mats in sink basins, and on countertops alongside the cook range, and under dish drying racks or baskets will cushion or absorb the impact noise caused by handling of dishes, pots and pans; further, such mats will tend to suppress the drumhead type amplification of impact noise by the hollow reverberant cabinets.

6. Cushion impact noise in the cabinets

Install soft rubber or cork tile on the shelves and back surfaces of the kitchen cabinets to minimize the impact noise caused by placing dishware or food supplies in the cabinets. The tiles will cushion or absorb the impacts caused by such chores.

Placing soft rubber bumpers or small strips of foam rubber on the inside edges of the cabinet doors will reduce the noise caused by the closing or slamming of the doors.

7. Apply vibration damping material

Sink basins equipped with a food waste disposer should be undercoated with a thick layer of a mastic type vibration damping material or other types of vibration dampers as illustrated in Fig. 3-9. Such treatment will suppress the vibration of the sink and resulting noise caused by operation of the disposer.
Damping treatment applied to the interior surfaces of the metal cabinets and tumblers of larger appliances, especially washing machines and dryers, would result in a noticeable reduction of noise due to cabinet vibration.

\section{Insta 11 acoustical lining}

Installing acoustical tile on the interior walls of the sink cabinet will minimize the noise buildup in the hollow reverberant enclosure. If space permits, this treatment should be applied to the cabinet that encloses the dishwasher or any other appliance.

9. Install exhaust fans on rubber mounts

Most ceiling exhaust systems use high speed, noisy propeller fans that are mounted rigidly to the ducts, which in turn are connected to the ceiling by means of the fan grilles. These structural paths carry the fan vibration to the ceiling, which acts like a large sounding board and amplifies the noise. You can reduce the noise level noticeably by following this three-step procedure: If possible, remount the fan on rubber grommets or spacers to isolate it from the duct, as shown in Fig. 4-9. In addition, try to break contact between the duct and the ceiling by inserting a soft sponge rubber gasket or spacers between the grille and the ceiling. Operate the fan at its lowest speed.

In the case of a hood type fan installed directly above the stove, it is unfortunately much more difficult to reduce the noise output because of the severe space limitations. In most situations, the only measures you can use to lower the noise are to operate the fan at its slowest speed, and to keep the oil and grease from accumulating on the fan blades and clogging up the pores of the filter pack. If it is at all possible to do so, install the fan on rubber mounts; this will reduce the noise due to hood vibration. 


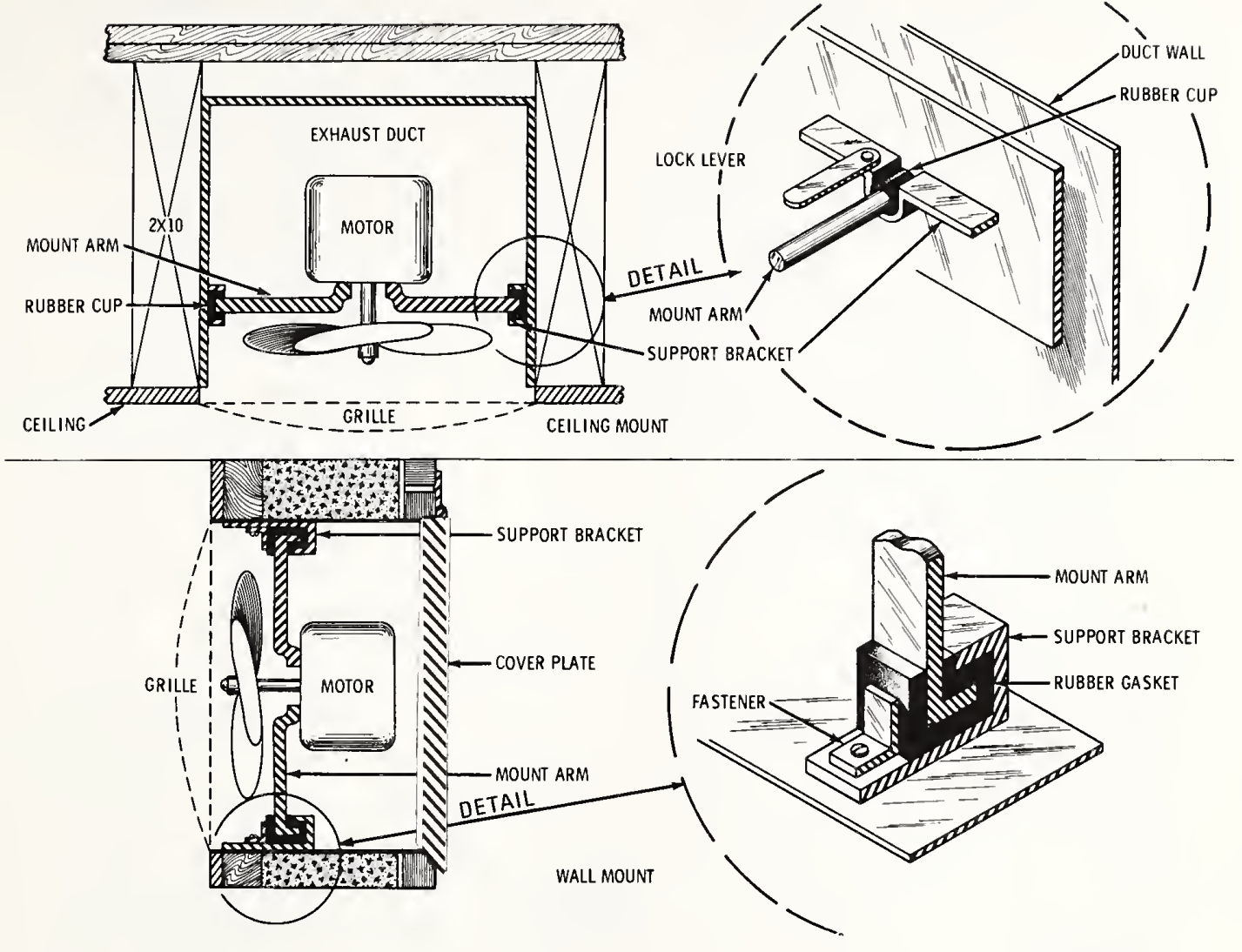

Fig. 4-9. PROPER INSTALLATION OF AN EXHAUST FAN

When replacement of a ceiling or hood exhaust fan is due, remember that squirrel-cage type fans are less noisy than propeller blade units, as illustrated in Fig. 3-2.

The ideal solution to avoid exhaust fan noise is to install a squirrel- cage fan on rubber mounts at the discharge or outdoor end of the duct instead of at the intake end in the kitchen ceiling. These measures combined with some acoustical lining near the fan and a flexible link between the duct and the ceiling would result in a relatively noiseless operation. These measures should be used in the installation of bathroom exhaust fans as well.

10. Install acoustic ceiling

The installation of an acoustic ceiling in the kitchen area will absorb a noticeable amount of noise. For ease of cleaning and maintenance, vinyl or membrane faced tile are available. Draperies, curtains and racks of exposed toweling also are good sound absorbers, particularly at high frequencies.

11. Install carpet or foam-backed tile Installation of foam-backed tile or an indoor/outdoor type carpeting, which is stain resistant, is an effective way of reducing impact noise caused by footsteps and dropped objects.

12. Quieting of large appliances

The application of basic noise-control principles in the early design stages of large appliances would eliminate much of the noise and disturbance such 
devices cause. This, of course, is an engineer's responsibility, and should have been incorporated in the design. The consumer can encourage such practices by giving preference to quiet appliances. Existing appliances can be quieted to some degree by applying the noise control measures illustrated in Fig. 4-10, and listed below.

(a) Insta11 ribbed neoprene or soft rubber pads under the legs of the units.

(b) Adjust screw-leveling legs to prevent units from wobbling.

(c) Tighten all loose parts and panels to prevent them from rattling. (d) Install soft rubber gaskets around pipe openings and along the perimeter edges of doors, of washing machines, dryers, and dishwashers to prevent noise leakage from inside the machines.

(e) With such units in operation, run your fingers lightly over the surfaces of the machines to find areas of greatest vibration. At these points install vibration damping materials on the inside surfaces. This will tend to suppress the vibration and reduce the noise output.

(f) Apply glass fiber board on the interior surfaces of the

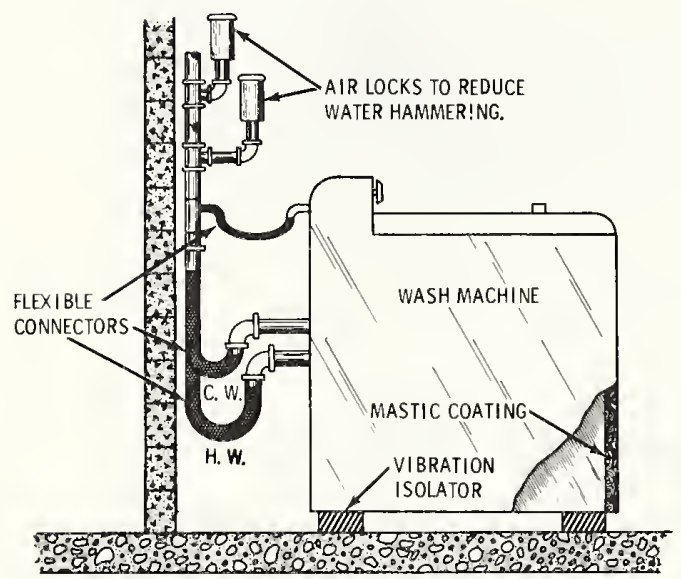

USE RUBBER PADS AND GASKETS

TO VIBRATION ISOLATE

APPLIANCE FROM CABINET

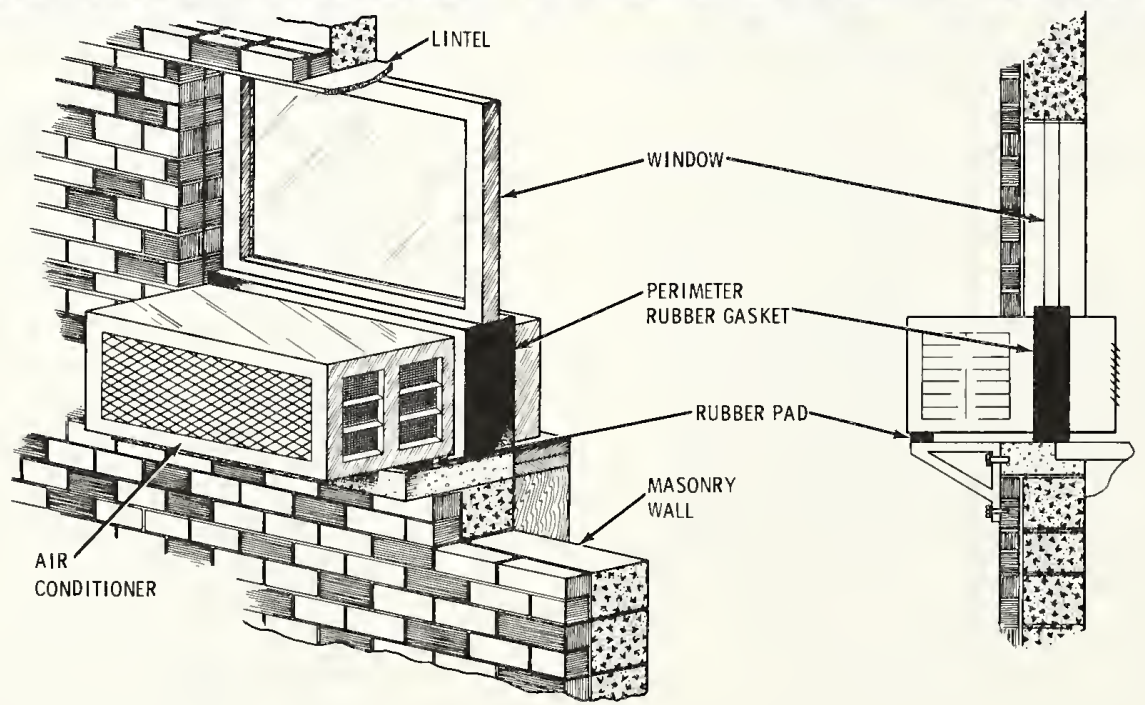

Fig. 4-10. PROPER INSTALLATION OF APPLIANCES FOR QUIET OPERATION 
cabinets to reduce the noise buildup in these reverberant enclosures.

(g) The more technically competent person might try mounting motors and pumps on resilient isolators and inserting rubber sleeves around pipes and conduits at points of support or contact with large cabinet surfaces.

Incorporation of the above acoustical measures will make a substantial reduction of the noise in the kitchen area.

\section{RECREATION OR PLAY ROOMS}

Other rooms of the home can be as noisy as the kitchen. These rooms are the family or recreation rooms, where members of the family, especially children, are permitted to watch television programs, listen to their stereo sets, play games or practice their music lessons. Such rooms tend to be somewhat reverberant and noisy because the wall and floor surfaces and furniture generally found in these rooms are made of hard materials designed to be stain resistant and durable. In addition, the rooms generally have wide open passageways, which permit the sound to travel to other areas.

Installing acoustic tile on the ceiling, drapery on the walls, and perhaps placing a few pieces of shag carpeting on the floor will reduce the noise buildup, and diminish distraction and annoyance.

While such measures will reduce noise levels in the recreation room, they do 1ittle to keep the noise from spreading to other areas of the home where a quiet and relaxing atmosphere may be desired even during daylight hours. The most effective measure to decrease the leakage of noise from rooms with open passageways is to install sound insulating doors, as was recommended in the case of the kitchen area.

However, some people, mothers in particular, might object to having rooms closed off for fear that they may not be able to hear what their children are doing in other parts of the house. Of course, when needed, doorways can always be kept open, but a more practical solution to this problem is to install an intercommunication system throughout the house. This electronic device will enable mothers to hear their children at will anywhere in the house without compromising the sound isolation that other members of the family might enjoy.

Noise in recreation rooms caused by playing such popular games as darts, table tennis, and billiards can be made less disturbing, without detracting from the fun, by placing a rubber pad behind the dart board, undercoating the tennis table with a vibration damping compound, and by placing rubber pads at the bottom of the billiard pockets and lining the ball return chutes with rubber or cork tile. Such treatment will cushion the impacts and muffle the resultant noise and distraction.

Earphones or headsets can be used when anyone wishes to listen to a sound system, radio or television set without disturbing other members of the household. This use, of course, does open the possibility that the listener unwittingly can turn the sound volume up to levels hazardous to his hearing. Some limit should be set, either by an attenuation pad inserted at the earphone outlet (which can be installed by a serviceman) or by marking a stop position on the volume control, beyond which it should not be turned.

Basement Recreation Rooms

In one-story homes, special attention must be paid to prevent the transmission of sound from recreation rooms located in basements to noise sensitive areas on the floor above. In many cases, recreation rooms are located directly below bedroom and study areas where privacy and quiet surroundings are most desired. The installation of a sound insulating ceiling assembly will alleviate such problems. A ceiling assembly which will provide adequate sound insulation under most circumstances would consist of the following construction, as illustrated in Fig. 4-11.

(a) If the floor joists are exposed, install a 3 inch $(7.6 \mathrm{~cm})$ thick layer of glass fiber insulation between the joists,

(b) attach metal resilient channels to the under edges of the joists. The channels should be installed so that they are perpendicular to the joists and spaced 2 feet $(60$ $\mathrm{cm})$ apart.

(c) Attach a 5/8-inch $(16 \mathrm{~mm})$ thick layer of gypsum board to the 


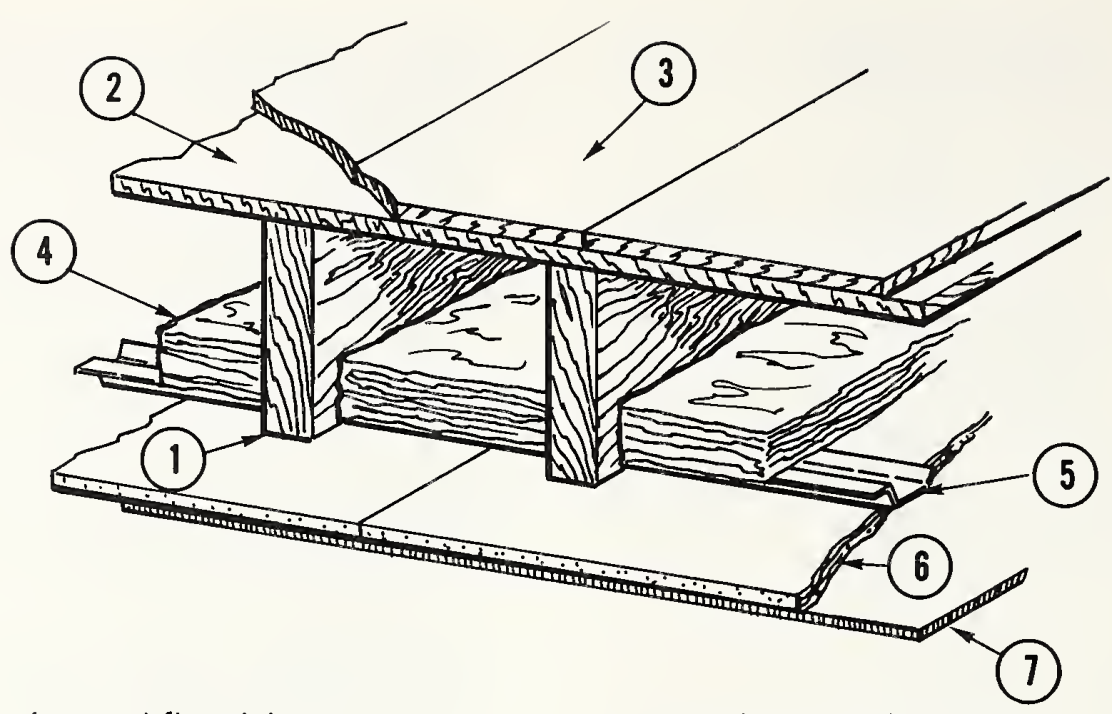

1. wood floor joists

2. wood subfloor

5. resilient metal channels

3. wood finish floor

4. glass fiber blanket, 3 in. thick

6. gypsum board, $5 / 8$ in. thick

7. acoustical tile

Fig. 4-11. DESIGN OF A SOUND INSULATING CEILING FOR A BASEMENT RECREATION ROOM

resilient channels with self-tapping screws.

(d) Provide a 1/4-inch (6 mm) clearance around the perimeter edge of the ceiling to avoid contact with the walls, and fill the space with a resilient non-setting caulk.

(e) To reduce excessive reverberation in the recreation room, cement acoustic tile to the gypsum board ceiling. As mentioned previously, the application of the acoustic tile will not improve the sound insulation of the new ceiling construction, but will only reduce the noise level in the recreation room.

Install a sound insulating door with proper gaskets at the entrance to the staircase leading to the basement recreation room. This measure will confine the noise to the recreation room area.

Install carpeting on the stairs to absorb both airborne noise and the impact noise of footsteps.

\section{CORRIDORS, HALLS, STAIRCASES}

In most apartments and homes, corridors or halls separate the noisy kitchen and family areas from the bedroom and study areas where a relatively high degree of privacy and quiet is desired. The most effective way of preserving this privacy is to install a solid core door at the entrance of the hall leading to the bedroom area. Such doors, when installed with proper gaskets, become very effective barriers against the noise escaping from the kitchen or family room areas of the home. In addition, installing a soft thick carpet in the hall and staircase will muffle the impact noise of foot steps and reduce reverberation thus leading to a quiet environment.

IMPROVING THE PRIVACY OF YOUR BEDROOM, STUDY AND BATH AREAS

Although interference with a person's sleep is least to be tolerated, especially at night, there are times when one desires quiet and privacy during the day. Areas requiring such privacy are the bedroom, study, and bathroom areas. Unfortunately, in most homes and apartments the lack of 
privacy in these areas is obvious. This arises from the use of the following building constructions and practices:

(a) thin, veneer, hollow-core doors,

(b) open air spaces under doors that provide for the circulation of return air, and

(c) the poor sound insulation provided by 1 ight interior walls throughout the home, particularly in bedroom areas.

The common practice of installing a forced-air ventilation system in some central closet location is responsible for the lack of privacy in homes and apartments. Since such a system often uses a central hall or corridor as the main air return duct, all other rooms bordering on this hall must vent the return air through open air spaces under the doors. Unfortunately, such

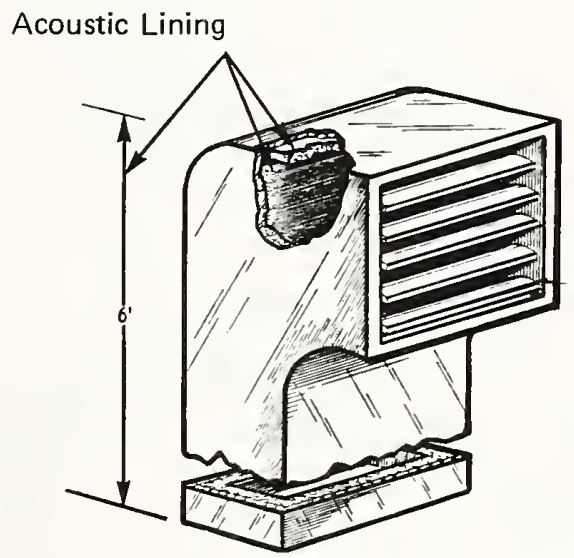

air paths become sound paths that nullify any privacy ordinarily provided by walls and doors.

The obvious solution to this problem is to:

(a) install solid core doors with perimeter gaskets and a drop closure,

(b) if possible, install separate return air ducts in each room. Sometimes this can be done in one-story houses, if the floor joists and ductwork are exposed in the area below. If this is not possible, install acoustically lined air transfer grilles or ducts, as illustrated in Fig. 4-12. Such ducts are commercially available.

For quiet operation: Use large size grille, $6 \mathrm{ft}$. length of acoustic lining in each duct run, rounded duct corners and stream-lined deflectors or wide-mesh grille face. Avoid sharp edges.

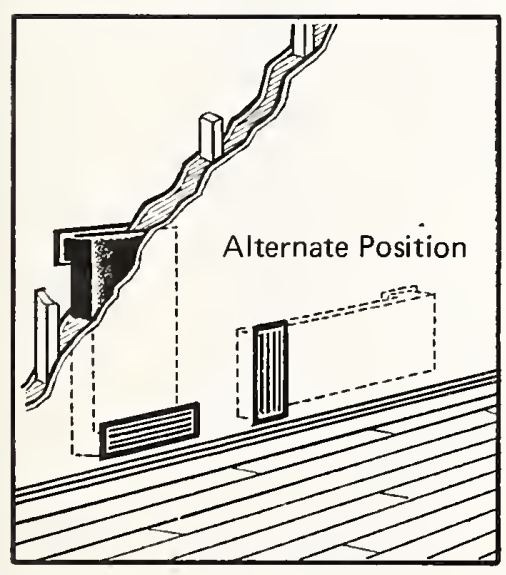

IN WALL

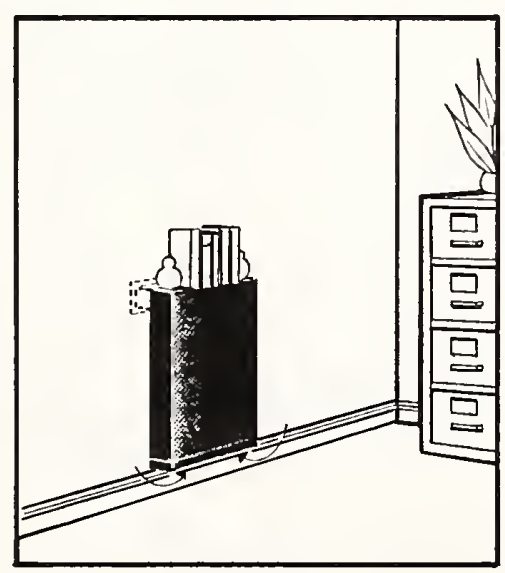

ON WALL

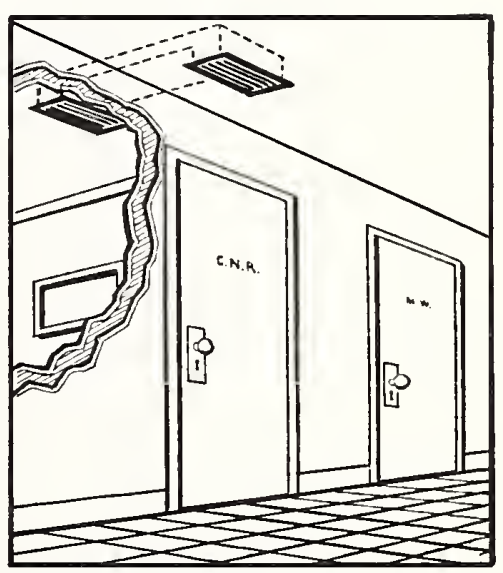

IN CEILING

Fig. 4-12. DESIGN AND INSTALLATION OF A QUIET AIR RETURN DUCT 
During construction of the house, similar return air ducts could have been built between the studding of the bedroom walls, by simply lining the inside surfaces of the wallboard with acoustical duct liner and providing an outlet grille at the top of the duct on the hall side of the wall, and an intake grille at the bottom of the duct on the bedroom side of the wa11.

If one is disturbed by conversation, TV sounds, etc., coming through the walls in bedroom areas, he should also look for noise leaks at the base of the walls and at electrical outlets.

The sound insulation of an existing wa11 may be improved by eliminating such sound leaks or by laminating another layer of wallboard, preferably to both sides of the wall. (See Fig. 4-13.)

\section{IMPROVING THE SOUND INSULATION OF WALLS}

A quantitative measure of the sound insulation provided by a wall or floor structure is the sound transmission loss. The sound transmission loss is defined as the number of decibels by which sound power randomly incident on a partition wall is reduced in transmission through the wall. This is illustrated in the left top diagram of Fig. 4-5, which shows a wall with an STL value of $30 \mathrm{~dB}$ reducing an incident noise level of $70 \mathrm{~dB}$ to a transmitted level of 40 $\mathrm{dB}$, a $30 \mathrm{~dB}$ reduction. In this case, the transmitted noise level is above the background noise and as a result is audible. The right diagram shows a $50 \mathrm{~dB}$ wall under the same noise conditions reducing the transmitted noise to an inaudible level, i.e., below that of the background noise. In this instance, the background noise is said to mask the transmitted noise. In the first example, the wall would not provide satisfactory sound insulation whereas in the second case, adequate insulation would be achieved under these conditions.

Thus, the satisfactory performance of the wall in a given situation hinges primarily on three factors: (1) the sound level on the source or noisy side; (2) the sound transmission loss of the wall; and (3) the background noise level on the receiving or quiet side of the wall.

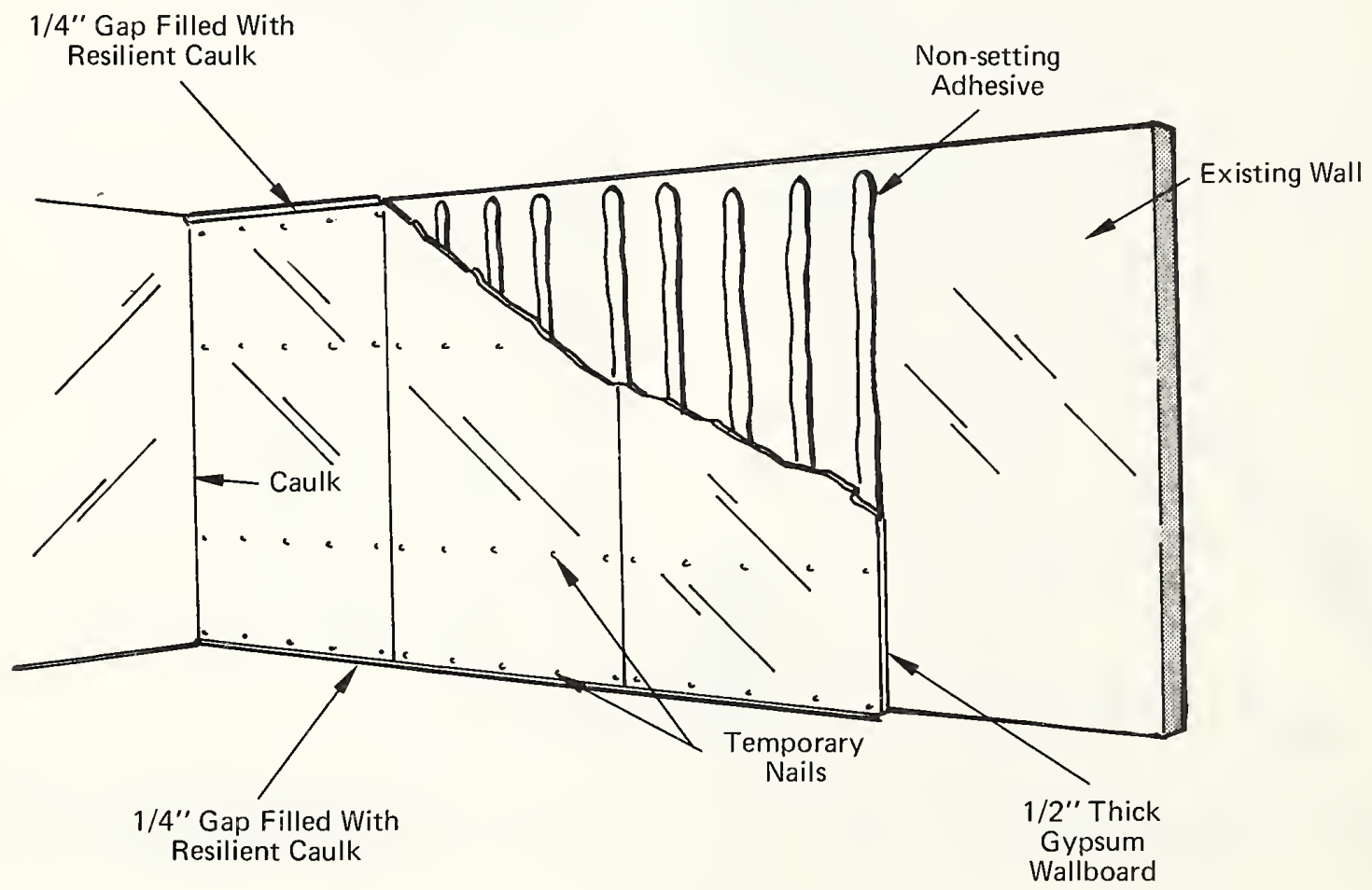

Fig. 4-13. IMPROVING THE SOUND INSULATION OF AN EXISTING WALL 
As we recognize from daily experience, the sound transmission loss differs for sounds of various frequencies. The low-frequency sounds of a passing truck travel through a wall structure much more readily than do the high-frequency sounds generated by chirping birds. In order to cope with this variation in a practical way, a frequency-weighted single number rating, called the sound transmission class (STC) was developed. The STC rating of a building structure is derived in a prescribed manner from the sound transmission loss values measured in sixteen frequency bands spaced between 125 and $4000 \mathrm{~Hz}$. The higher the STC rating of a partition, the greater is its effectiveness as a sound insulator. Table 4-1 shows the performance of various wall structures with different STC ratings described in terms of their approximate effectiveness against intruding speech.

Due to conventional building materials and construction, most interior walls within a typical home or apartment would have STC ratings between 30 and 35 . The first five entries in the bibliography at the end of this Guide contain architectural illustrations of various wall and floor structures covering a wide range of STC ratings.
Many walls, especially party walls separating apartment units, are particularly vulnerable to noise leakage. The installation of back-to-back electrical outlets, medicine cabinets, and master TV outlets are common causes of noise leakage. openings around pipe penetrations and duct work are also causes of noise leakage. Cracks at the ceiling and floor edges of walls caused either by settlement or poor construction allow the transmission of noise from one space to another.

The sound insulation of many such walls can be improved simply by eliminating the noise leaks and flanking paths.

(a) Sealing holes and cracks: (see Fig. 4-14)

All holes or openings around plumbing fixtures, piping and drain pipes should be enlarged slightly to provide a clearance around the pipe. The opening should then be filled with a fiber glass packing and sealed completely with a resilient caulk. This same treatment should be used in openings around ducts, grilles and electrical outlets.

TABLE 4-1

SOUND TRANSMISSION CLASS (STC) FOR VARIOUS WALL STRUCTURES

STC

Rating

25

30

35

40

45

50

55
Shouting barely audible

Privacy Afforded

Normal speech easily understood

Norma1 speech audible but not intelligible

Loud speech audible and fairly understandable

Loud speech audible but not intelligible

Loud speech barely audible

Shouting not audible

\section{Wa11 Structure}

$1 / 4 "$ wood panels nailed on each side of $2 \times 4$ studs

$3 / 8^{\prime \prime}$ gypsum wallboard nailed to one side of $2 \times 4$ studs

5/8" gypsum wallboard nailed to both sides of $2 \times 4$ studs

Two layers of 5/8" gypsum wallboard nailed to both sides of $2 \times 4$ studs

Two sets of $2 \times 3$ studs staggered $8^{\prime \prime}$ on centers fastened to $2 \times 4$ base and head plates with two layers of $5 / 8^{\prime \prime}$ gypsum wallboard nailed on the outer edge of each set of studs

$2 \times 4$ wood studs with resilient channels nailed horizontally to both sides with 5/8" gypsum wallboard screwed to channels on each side

3-5/8" metal studs with $3^{\prime \prime}$ layer of glass fiber blanket stapled between studs. Two layers of 5/8" gypsum wa11board attached to each side of studs. 


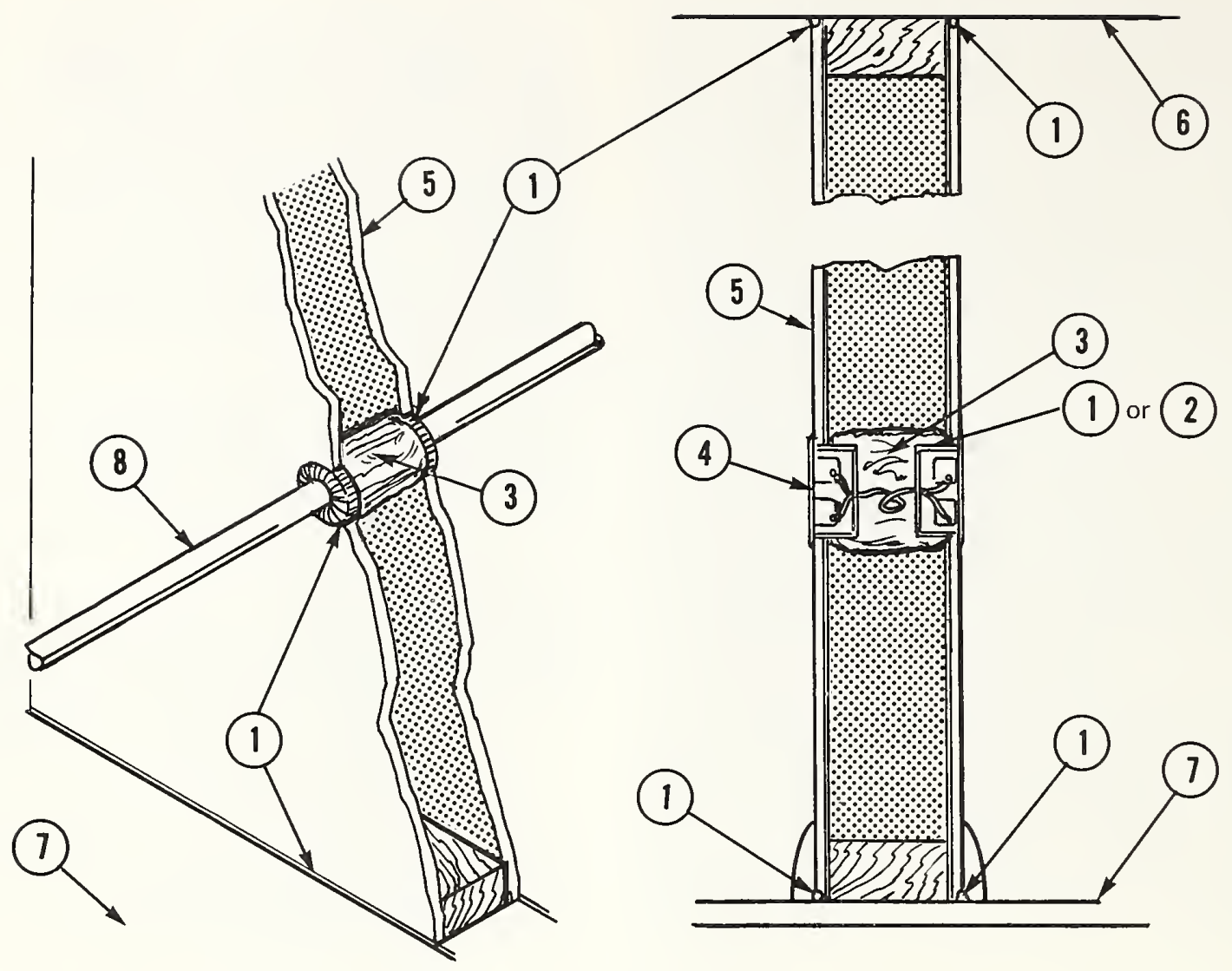

1. Caulk

3. Glass Fiber Packing

5. Wall

7. Floor

2. Lead Wrap

4. Electrical Outlets

6. Ceiling

8. Pipe

Fig. 4-14. SEALING COMMON NOISE LEAKS IN EXISTING WALLS

Settlement cracks at the ceiling edge of a wall should be sealed with a resilient caulk, preferably applied under pressure with a caulking gun. Cracks at the floor edge of the wall should be handled in the same manner, although it might require removing the toe molding and base boards to do a thorough job.

(b) Sealing leaks behind back-to-back outlets and cabinets: (see Fig. 4-15)

If you hear your neighbor's noise coming through an electrical outlet, remove the cover plate and examine the opening. If necessary, remove your outlet box, pack glass fiber wadding around your neighbor's outlet, and insert a 1/16-inch ( $1 \mathrm{~mm}$ ) thick sheet of lead to form an airtight barrier wall. Reinstall your outlet box, fill the remaining voids with the glass fiber and caulk and replace the cover plate.

If the party wall is of wood stud construction, you may be able to rewire and install your outlet in the adjoining stud space. Fill the voids around both outlets with the glass fiber wadding and seal the former opening with a piece of wallboard.

Noise transmission through recessed medicine cabinets can be 


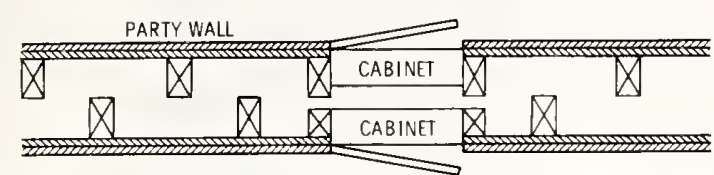

AVOID RECESSED BACK-TO-BACK MOUNTING OF CABINETS IN PARTY WALLS

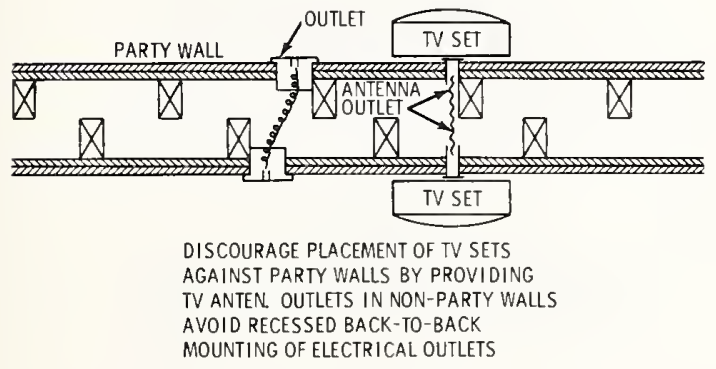

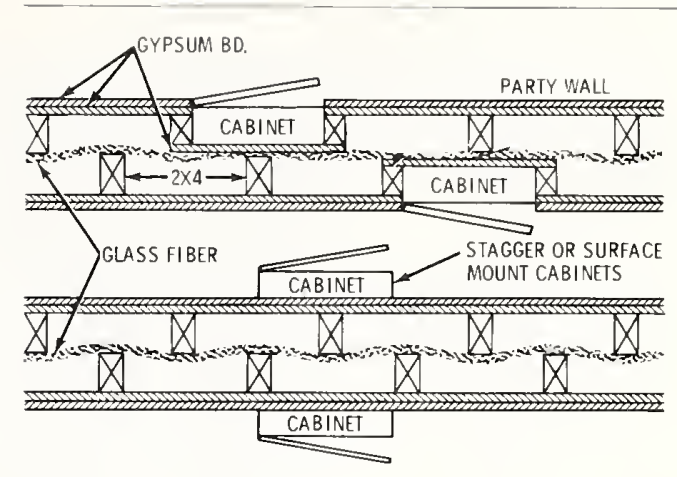

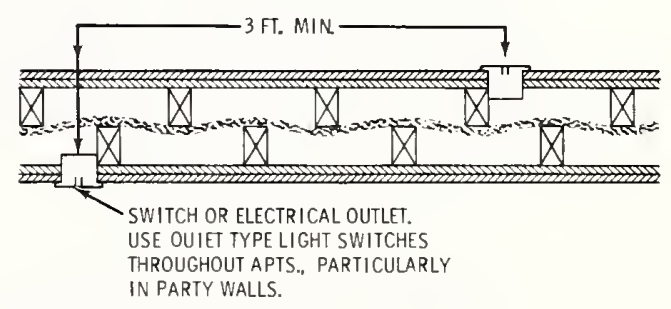

Fig. 4-15. PROPER INSTALLATION OF CABINETS AND ELECTRICAL OUTLETS IN WALLS

handled in a similar manner. However, if space permits, you may be able to insert a gypsum board or a sheet of plywood as a barrier between the two cabinets. In this case you should caulk the perimeter edges of the intervening barrier before installing your cabinet.

(c) Eliminating flanking paths: (see Fig. 4-16)

The chief flanking paths in homes and in apartment buildings are open ceiling plenums, attic spaces, basement areas and crawl spaces which span freely across dwelling units or adjacent rooms. Such areas should be completely subdivided with full height partitions or barriers installed directly above and below the party walls separating the dwelling units or rooms in question.

If the above recommendations fail to improve the sound insulation of the party wall sufficiently, then modifications to the existing wall must be made. Where only a moderate degree of sound insulation is required, laminate a $1 / 2^{\prime \prime}$ (13 min) thick layer of gypsum wallboard preferably to both sides of the existing wall with a resilient non-setting adhesive, as illustrated in Fig. 4-13. In addition, provide a $1 / 4^{\prime \prime}$ (6 $\mathrm{mm}$ ) clearance along the perimeter edges of the wallboard and fill the space with a non-setting caulk compound.

A much higher degree of privacy would require the attachment of a wall of special design to the existing wall. The new wall, as shown in Fig. 4-17, would be constructed as follows:

(a) Fasten wood furring members, spaced $16^{\prime \prime}(40 \mathrm{~cm})$ on centers vertically, to the existing wall.

(b) Nail resilient metal channels perpendicularly to the furring members; channels should be spaced $24^{\prime \prime}(60 \mathrm{~cm})$ apart.

(c) Fill the space between the existing wall and the channels with a glass fiber blanket. 

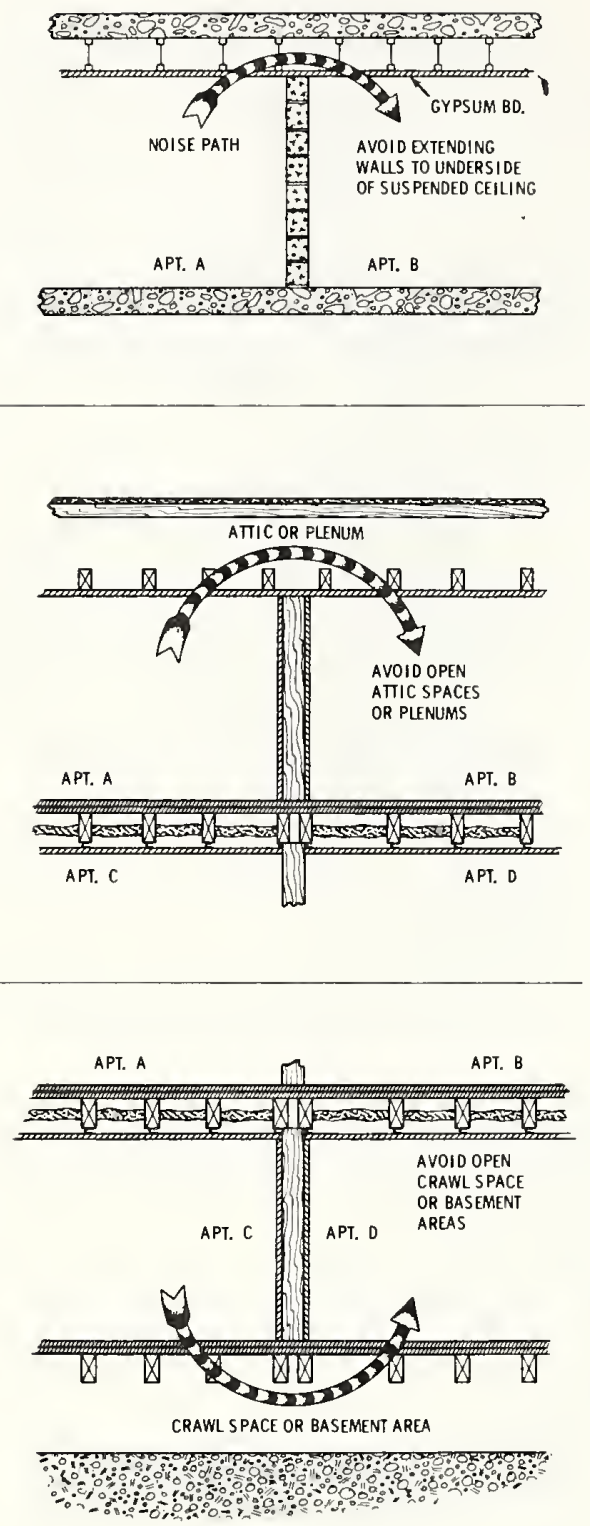
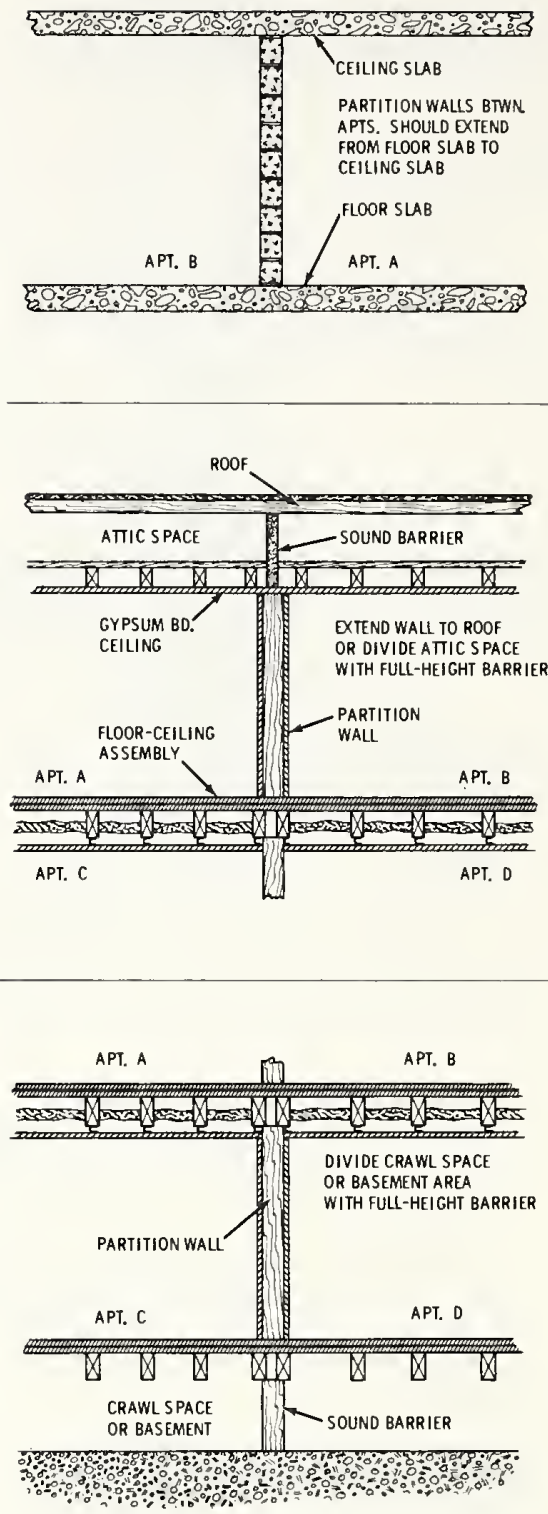

Fig. 4-16. METHODS OF CONTROLLING FLANKING SOUND TRANSIMISSION PATHS 


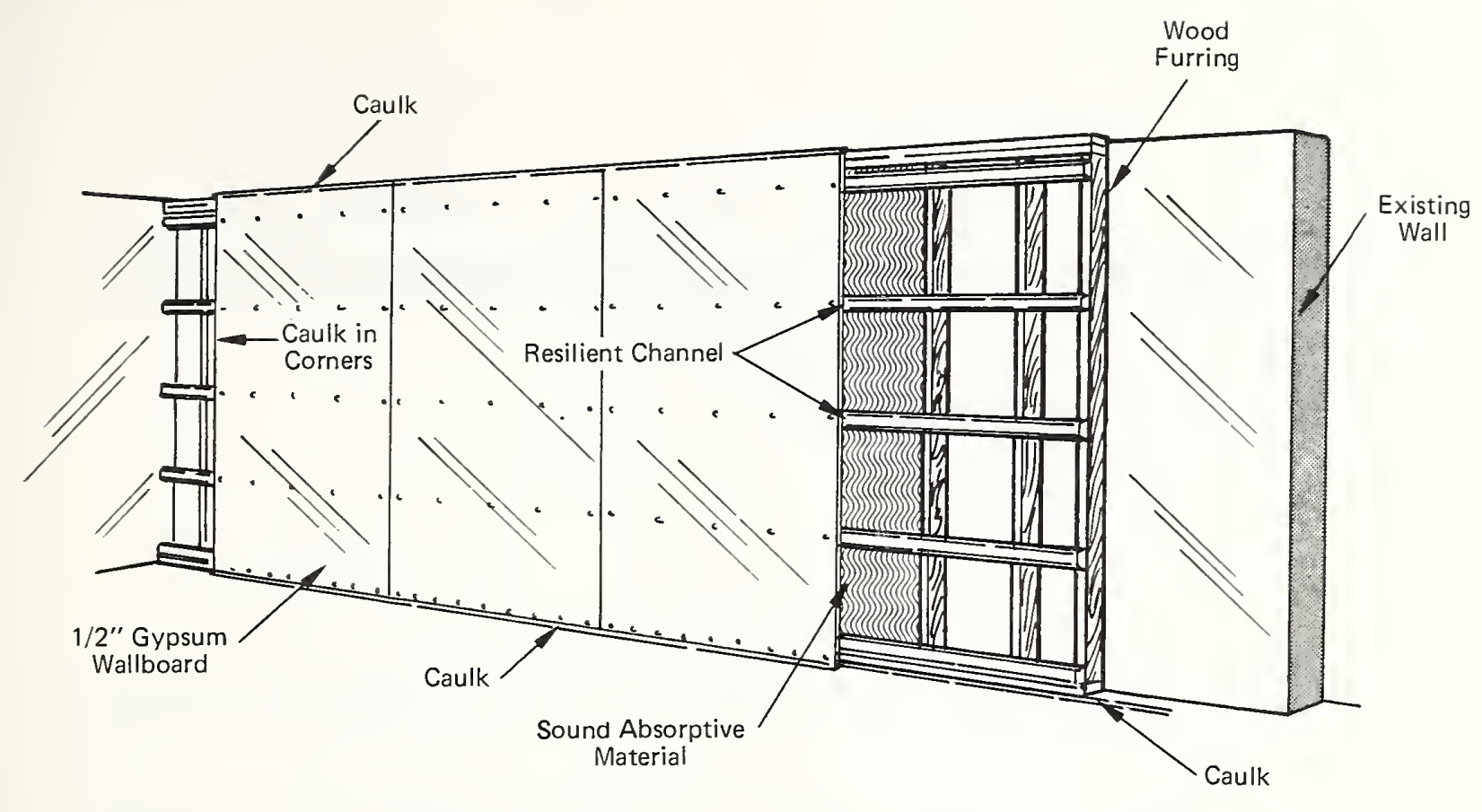

Fig. 4-17. METHOD OF OBTAINING A SUBSTANTIAL INCREASE IN SOUND INSULATION FROM AN EXISTING WALL

(d) Attach 1/2" (13 mm) thick gypsum wallboard to the metal channels with self-tapping screws.

(e) Provide a 1/4" (6 mm) clearance around the perimeter edge of the wallboard to avoid restraining contact witin adjoining surfaces of the room; fill the clearance gap with a resilient non-setting caulk.

IMPROVING THE SOUND INSULATION OF FLOOR-CEILING CONSTRUCTIONS

A floor-ceiling construction separating two rooms or apartment units will provide adequate sound insulation or privacy, if it can reduce the intrusion of conversational or TV sound and footstep or impact noise to non-disturbing levels.

If you are disturbed by the transmission of only footstep or impact noise from the room above, the installation of a soft thick carpet, preferably on a felt or foam rubber pad on the floor above will eliminate the problem in most cases.
If both footstep and conversational or TV noise from the room above annoy you, modifications to the existing ceiling must be made to remedy the problem. A gypsum board ceiling supported on resilient channels should be attached to the existing ceiling. This second ceiling, which is illustrated in Fig. 4-18 would be constructed as follows:

(a) Attach wood furring members, spaced 16" $(40 \mathrm{~cm})$ on centers to the existing ceiling.

(b) Nail resilient metal channels perpendicularly to the furring members; channels should be spaced $24^{\prime \prime}(60 \mathrm{~cm})$ apart.

(c) Fill space between the existing ceiling and the channels with a glass fiber blanket.

(d) Gypsum wallboard, 1/2" (13 mm) thick should be fastened with self-tapping screws to the resilient channels. 


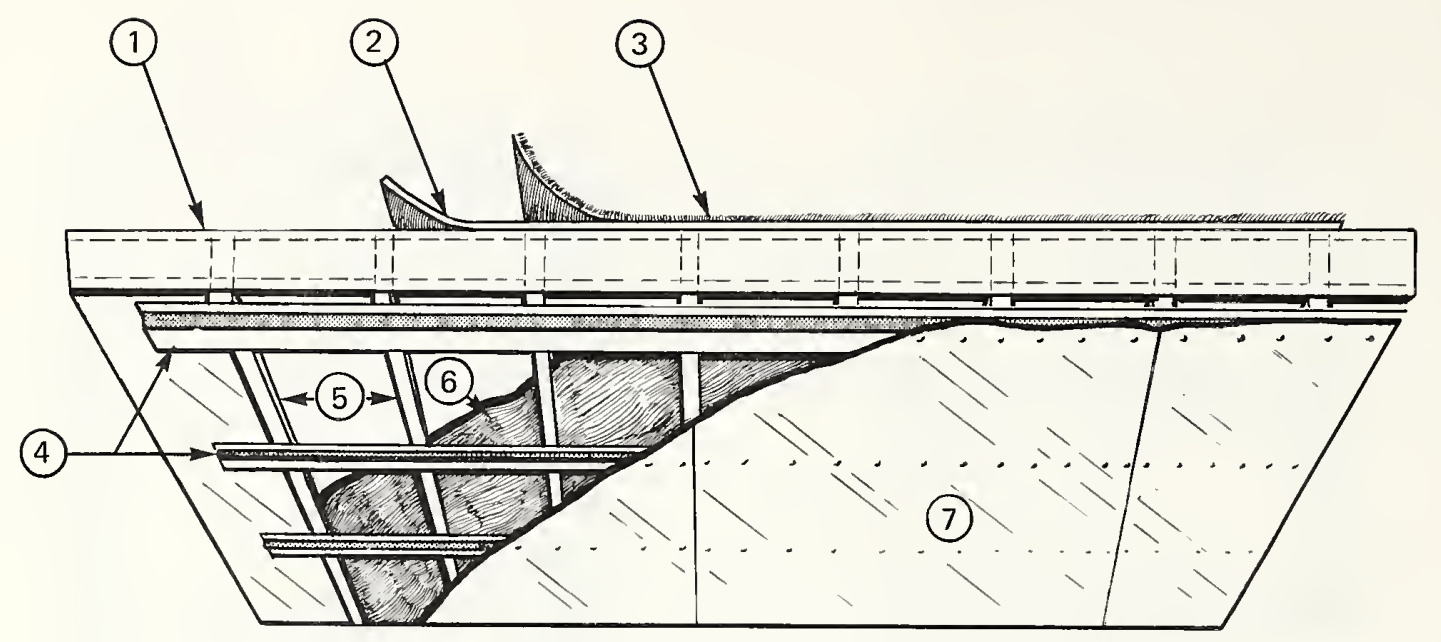

1. Existing floor-ceiling structure

5. Wood furring

2. Pad

6. Glass fiber pad

3. Carpet

7. Gypsum ceiling board

4. Resilient metal channels

\section{Fig. 4-18. METHOD OF INCREASING THE AIRBORNE AND IMPACT SOUND INSULATION OF AN EXISTING FLOOR-CEILING STRUCTURE}

(e) Provide a $1 / 4^{\prime \prime}(6 \mathrm{~mm})$ clearance around the perimeter elige of the wallboard ceiling to avoid contact with the walls, and fill the gap with a resilient, non-setting caulk.

This ceiling assembly should not only eliminate the airborne noise transmission, but should make a substantial reduction in the footstep noise level. However, if additional reduction in footstep noise is desired, then a carpet and pad placed on the floor above will solve the problem.

\section{CONTROL OF FLOOR SQUEAKING (See Fig • 4-19)}

Squeaking floors cause considerable irritation because they generally produce high-pitched noises to which the person with average hearing is highly sensitive. Although the squeaking floor problem is more commonly found in homes of wood frame construction, it occurs quite often in apartment buildings with concrete floors surfaced with wood block or strip flooring.

\section{Causes of Floor Squeaking:}

In general, floor squeaks are caused by the rubbing or sliding of sections of finished flooring over the subfloor. Movement between adjoining wood blocks, strips or sections of both sub-flooring and finish floor also cause squeaking. This problem usually is the result of faulty workmanship, the use of defective materials or poor structural design, such as:

(a) poor nailing: The most frequent cause of floor squeaking is the failure to nail the sub and finish flooring materials tightly and securely together and to the supporting joists or structural floor. This failure results from the use of undersize or improper nails, excessively wide spacing of the nails and faulty nailing practices in general.

(b) defective materials: Another cause of floor squeaking is the use of warped or twisted floor joists, buckled or bowed subfloor sheathing, or wood block or strip

flooring with defective tongues and grooves. When layers of such materials are fastened together, air-pockets or gaps form between them resulting in poor support. This allows the materials to rock, move or deflect causing the squeaking under foot. 

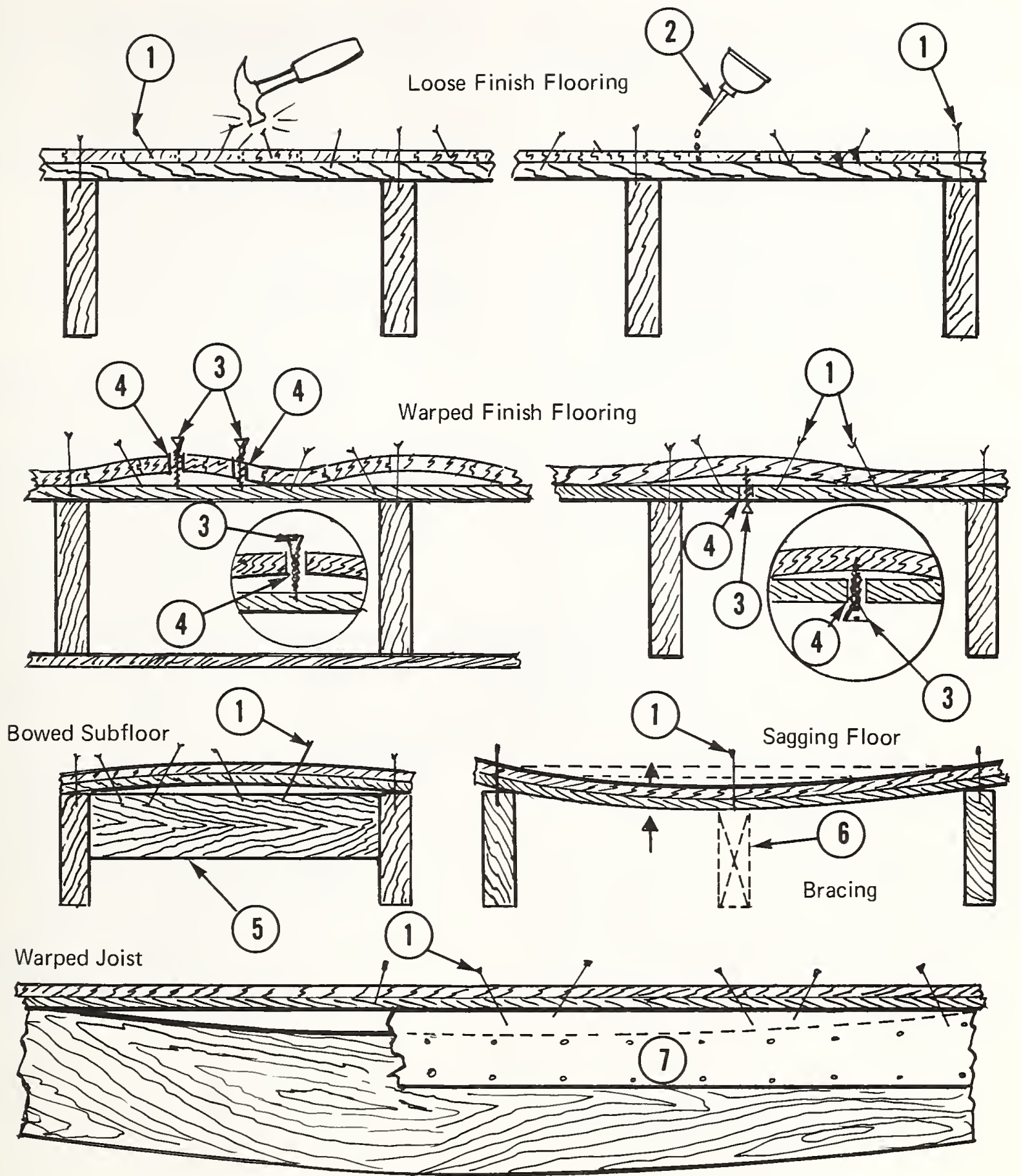

1. Toe-nail with ring nails

5. Insert cross brace

2. Apply mineral oil in grooves

6. Insert additional joist

3. Tighten wood screws

4. Drill clearance hole for screw

7. Butt long straight board against subfloor and nail to joist

Fig. 4-19. CAUSES AND REMEDIES FOR SOUEAKING FLOORS 
The installation of dry flooring in damp areas often results in squeaking floors because the absorption of moisture by the flooring causes it to swell or buckle, especially if no allowances were made for expansion.

(c) poor structural design: Although a floor joist system may be adequately designed in terms of structural and load requirements, it may deflect sufficiently under foot traffic to permit movement of the surface flooring; this generally occurs when either the depth of the joist is too shallow or the spacing between joists is too wide for a given floor span.

(d) absence of a sheathing layer: For reasons of economy, builders no longer adhere to the long-standing practice of inserting a layer of building paper between the sub-flooring and the finish flooring. This omission is common in current floor construction and makes floor squeaking caused by rubbing and sliding contact between the floor surfaces a much more serious problem in modern housing.

Methods of Avoiding Floor Squeaking in New Construction:

The problem of floor squeaking can be avoided by using properly designed floor structures, good quality materials, and proper installation techniques. With regard to floors consisting of wood blocks laid in mastic over wood or concrete subfloors, the manufacturer's instructions should be followed closely relative to the preparation or priming of the subfloor, application of the mastic and the laying of the wood blocks.

In general, floor squeaking can be minimized by observing the following construction techniques.

(a) Use straight, true, properly seasoned flooring materials and joists.

(b) Build a rigid well-constructed and properly supported floor system.

(c) Maintain proper moisture content of flooring prior to installation. (d) Insert building paper or felt between finish and subfloor layers to eliminate rubbing or sliding contact.

(e) Employ good nailing techniques, with emphasis on proper spacing and angle.

Ways to Remedy Existing Problems:

If you have floors that squeak, the following recommendations should help you correct the problem.

(a) To locate the problem, have someone walk over the squeaking portion of the floor while you examine the surface closely. If you hold a flashlight so that its beam grazes the floor surface, it is quite easy to see the motion.

(b) If you see no motion on wood block or strip flooring, the squeaking may be due to slippage between the tongues and grooves. Apply mineral oil sparingly at the seams between adjacent floorboards to lubricate the tongues and grooves where the slippage is taking place.

(c) Loose or bowed finish flooring may be securely fastened to subflooring by surface nailing with ring type or serrated nails into the subfloor and preferably the joists. Recess the nail heads and fill the hole with a wood sealer that matches the floor color. If the nails tend to split the flooring, predrilling a very fine hole through the finish floor before nailing will eliminate the problem.

(d) If the floor joists are exposed, and the finish flooring is warped, you can drill clearance holes through the subfloor and drive wood screws through the holes into the finish floor. The screws will draw the two layers of flooring tightly together.

(e) In places where the joists are warped or bowed, gaps will occur between the subflooring and the joists. Inserting wedges or shimming material tightly in the gap usually will remedy the problem. If the gap is very long, 
a 2 " $x$ 4" brace pressed up against the subfloor and nailed securely to the warped joist will provide adequate support.

(f) Excessive deflection of the structural floor due to lightweight or widely spaced joists may be corrected by the insertion of a fer extra joists, if the under surface of the floor is exposed. However, if there is a finished ceiling below the floor, cross- beaming with support columns may be the most expedient corrective measure.

CONTROL OF STAIR SQUEAKING (See Fig. 4-20)

As in the case of floor squeaking, the problem of stair squeaking can be avoided by using a properly designed structure, good quality building materials, and proper construction techniques. As you will notice, the causes and remedies of the problem also are similar to those associated with the problem of floor squeaking.

Stair squeaking may be caused by:

(a) loose or warped treads (the flat horizontal steps), or

(b) the binding and rubbing of the treads against the top or bottom edges of the risers (the upright or vertical boards).

\section{Renedies :}

(a) If the treads are loose, they should be nailed securely to the center and side stringers (the saw-tooth framework that supports the staircase).

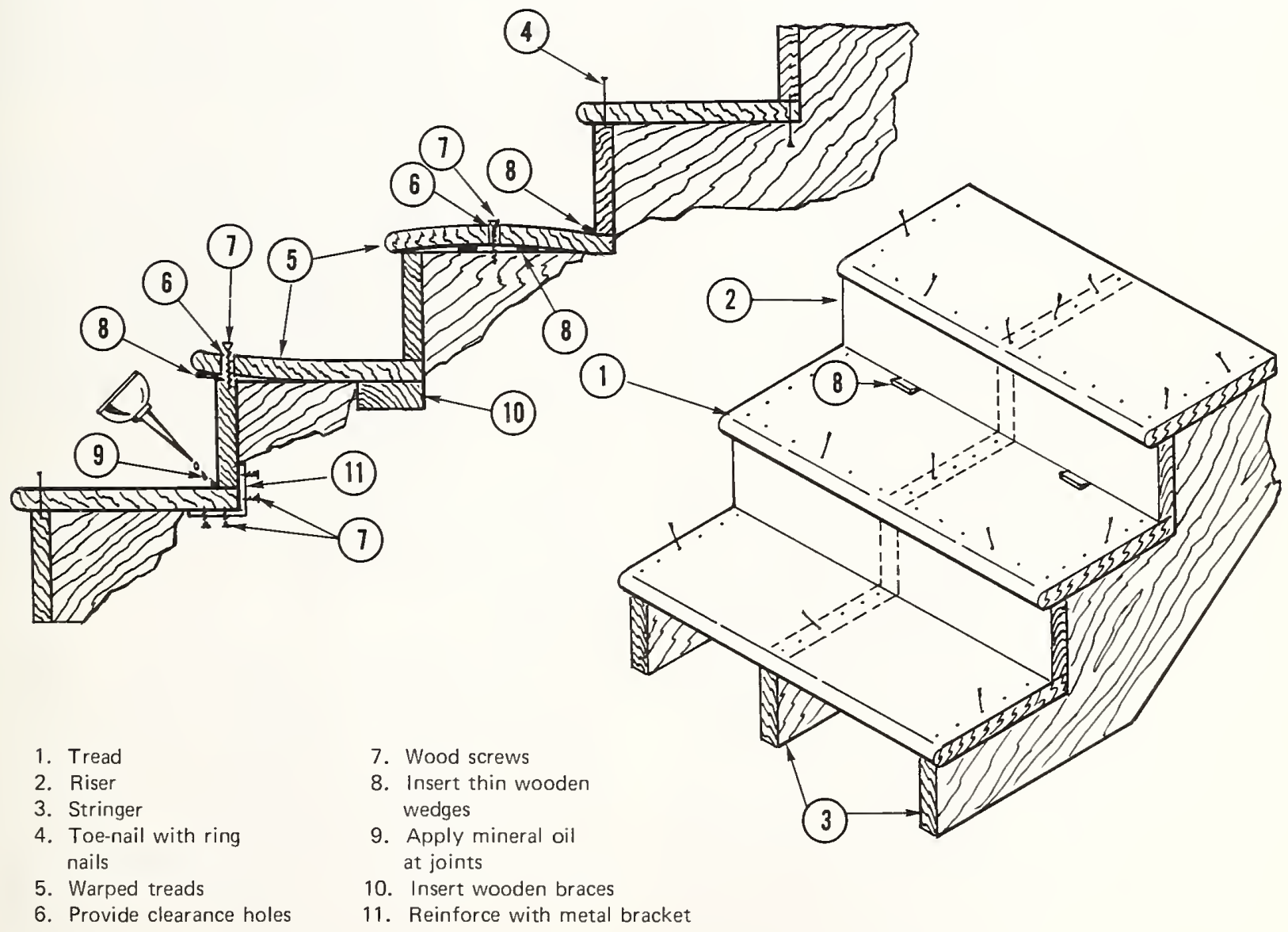

Fig. 4-20. TECHNIQUES FOR THE CONTROL OF STAIR SOUEAKING 
(b) If the treads are warped, inserting wedges between the tread and stringer usually will eliminate the rocking motion of the tread when it is stepped on. Drilling clearance holes through the treads, where they are supported by the stringer, and driving long wood screws through the holes into the stringers will pull a warped tread down tightly onto the stringer.

(c) Lubricating the top and bottom edges of the risers with mineral oil or silicone spray often will remedy the squeaking problem.

(d) Sometimes nailing the front edge of the tread to the riser or inserting thin wedges at the bottom of the riser will correct the squeaking problem.

(e) If the back of the stairs is accessible, the back edges of the treads may be nailed to the risers. Additional bracing also may be provided by fitting a $2^{\prime \prime} x$ 4" brace between the stringers, pressing it up against the bottom edge of a riser and nailing it securely to the stringers.

CONTROL OF HEATING, VENTILATING AND AIR-CONDITIONING SYSTEM NOISE:

(In Basement, Attic and Central Closet Installations)

The noise problem associated with heating, ventilating and air- conditioning systems is due primarily to the current trend of installing, in homes and apartments, small compact units driven by noisy high-speed, motor-coupled blowers. The large return grilles, which usually are coupled with short unlined ducts to the blower intake port and the open corridors that serve as centralized air-return ducts, aggravate the problem.

The problem reaches serious proportions when, for economic reasons, such units are installed in a closet centrally located in a house or an apartment. The typical closet provides virtually no sound attenuation because of its light-frame construction, louvered door, and large return grille. Thus the noise escapes with undiminished intensity and spreads throughout the home or apartment.
Homeowners and tenants alike often complain that their sleep is seriously disturbed throughout the night because of the noisy equipment. The noise also is a constant source of irritation and annoyance to them even during their leisure hours. However, they have no choice but to tolerate the noise or else suffer the discomfort of a poorly heated or air-conditioned dwelling, if they turn off the equipment.

\section{Causes of the Noise}

Outdoors

(a) Compressor: The noisiest component of most air conditioning systems is the compressor assembly. It consists of a compressor, motor and fan encased in a metal cabinet that is usually installed outside of the dwelling. As a consequence, it frequently disturbs the neighbors more than the home owner, especially if the neighbors sleep in bedrooms with open windows that overlook the compressor unit.

(b) The most objectionable noise is the cyclic operation of the compressor, in particular the low-pitched starting groan of the motor. Blade-frequency noise of the fan and high-pitched flow noise add to the annoyance. Occasionally, a slipping fan belt will produce a high-pitched screech, especially as the unit starts up.

(c) The reflection of the sound waves between the walls of adjacent houses builds up the noise level.

Indoors

(a) Motor and Blower: In a typical installation, the high-speed motor and blower, which generally are coupled together, are the main sources of noise. The mechanical noises of bearings, brushes and switches and the siren-like "blade-frequency" noise of the blower are transmitted throughout the dwelling both by air through the duct passages and by vibration of the ductwork and supporting walls or floor structures. The "blade frequency" noise of the blower may be determined by 
multiplying its speed (number of revolutions per minute), by the number of blower blades and dividing the product by 60 .

(b) Noise Resulting from Air Flow and Turbulence: Ventilation systems using high-speed blowers are characterized by high air-flow velocities. High velocity air flowing past obstacles in the duct air stream (sharp corners, ragged joints, dampers and grilles) generates a considerable amount of turbulence. However, the highly turbulent air flow that usually occurs at the discharge port of the blower is the predominant cause of duct vibration in most ventilation systems.

(c) Rigid Mounting of the Equipment: In most installations, the heating and air-conditioning equipment is mounted directly on the floor with all ducts coupled directly to wall and floor structures. As a consequence, the wall and floor structures are set into vibration and radiate the noise with increased intensity throughout the dwelling.

(d) Central Air Return Duct: The installation of the central return duct of a typical home size unit has two serious shortcomings. The return duct usually is coupled to the blower by a short- length, unlined duct with a relatively large cross-sectional area. As a result, the grille end of the duct radiates the noise from the motor-blower and air turbulence with undiminished intensity. Since there often are no air return ducts in individual rooms, entrance doors are undercut approximately 1 inch $(2.5 \mathrm{~cm})$ at the bottom to provide passage of the return air and thus complete the circulation. Unfortunately, the large air gaps under the doors are extremely efficient flanking noise paths which allow the noise to intrude into bedroom or other rooms where privacy is most desired.

If you are planning to build a home, insist that the builder installs both supply and return ducts in all bedroom and study areas and selects quiet heating and air conditioning equipment. Equipment should be selected on the basis of low noise output. The more progressive manufacturers provide sound power ratings of most types and sizes of equipment they market. Such ratings, which frequently contain sound power levels in various frequency bands under different load conditions, are useful for acoustical design purposes. A few salient points worth remembering are:

(1) It is less expensive to install quiet equipment than to reduce the noise output of a cheaper unit by costly acoustical treatment or construction.

(2) Centrifugal or squirrel-cage fans are less noisy than vaneaxial propeller fans, all other factors being equal.

(3) For a given air flow capacity, large-diameter, slow-speed, beltdriven blowers are substantially less noisy than small-diameter, high-speed motor-coupled blowers.

(4) Basement or slab-on-grade locations, far removed from living quarters, are preferred for the typical heating and cooling equipment installations. Attic locations should be avoided.

(5) For purposes of noise control, install equipment that is designed to meet the building's heating and cooling requirements, instead of installing smaller units which must labor continuously at maximum speed in order to meet the building's minimal demands.

Although the above advice may be useful to the potential homebuilder, it unfortunately offers no comfort to the tenants who have a noisy heating and air-conditioning system in their home or apartment.

If you have a noisy heating and ventilation system, check the following possible causes of the noise and respective remedies.

Remedies: (see Fig. 4-21)

Compressor:

(a) If necessary, relocate the compressor as far as possible 


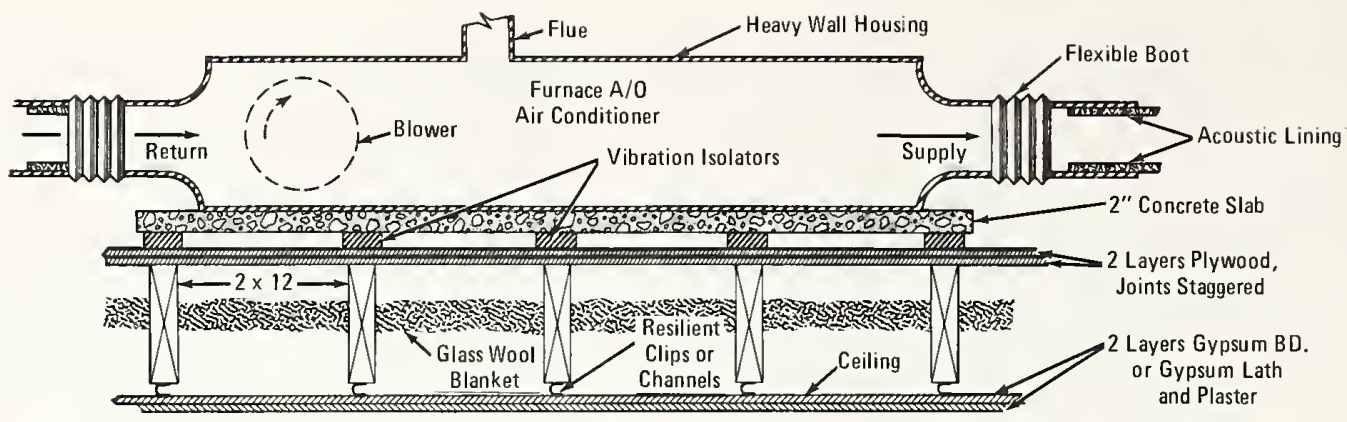

\section{ATTIC INSTALLATION}
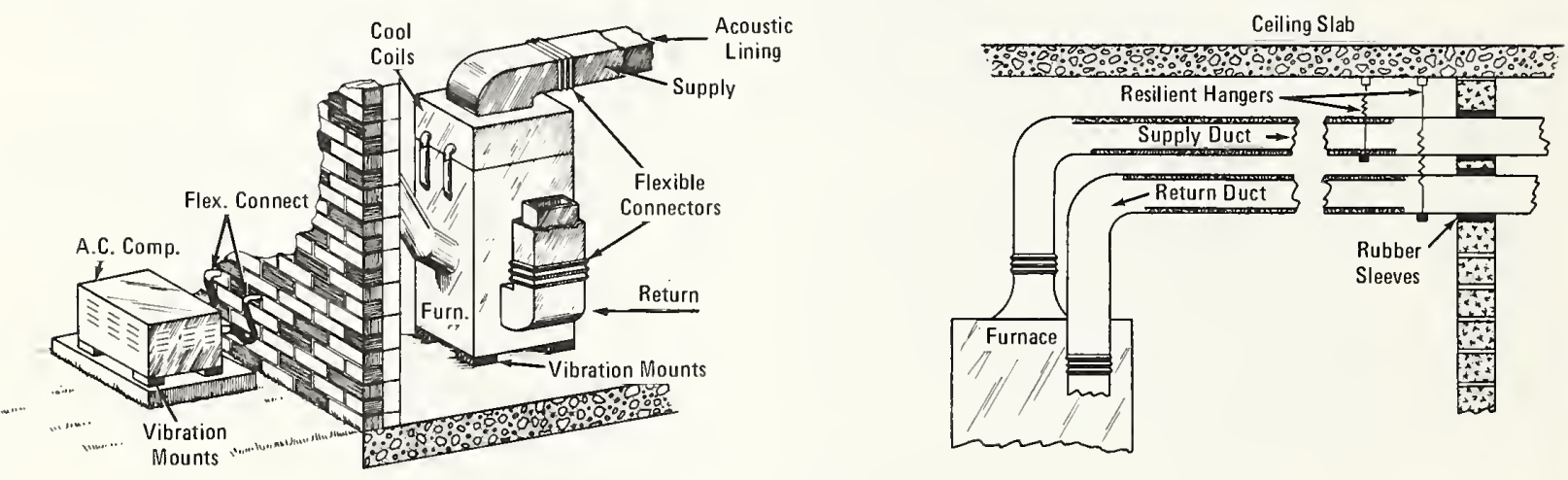

BASEMENT INSTALLATION

Fig. 4-21. TECHNIQUES FOR THE CONTROL OF NOISE FROM EXISTING ATTIC OR BASEMENT INSTALLATIONS OF FURNACES AND AIR-CONDITIONING UNITS

from the bedroom areas of your house and those of your neighbor's house as we11. An ideal location for the installation of compressors would be in the area between face-to-face kitchens of adjacent houses, especially if they are of rambler-type or one story construction.

(b) Insta11 the compressor on resilient mounts, preferably on a flat, level concrete slab that is at least 1 foot $(30 \mathrm{~cm})$ away from the exterior wall of the house.

(c) Install rubber sleeves or collars around all piping, refrigerant lines and conduits at points of support or penetration through the exterior wall of the house, to prevent the transmission of compressor vibration to the building structure. (d) Lubricate the bearings of the cooling fan, clean the blades and balance the unit. You can balance the fan simply by spinning it by hand and marking the bottom blade when it stops. If the same blade stops at the bottom following two or three spins, attach strips of adhesive tape to the opposite blade until balance is restored.

(e) Reduce blower speed, if possible and clean protective screen to ensure adequate air flow. Tighten and align fan belts and pulleys.

(f) Tighten loose parts or panels to prevent rattling or vibrational resonance.

(g) Install sound barriers or partial enclosures around the compressor to shield or 
direct the noise away from bedroom or other noise sensitive areas.

Motor and Blower:

(a) Lubricate or oil bearings.

(b) Clean blower blades and balance the unit as described in item (d) above.

(c) If blower is belt driven, replace worn or frayed belts, adjust tension on belt to prevent belt resonance or "drumming" and check pulley alignient.

(d) Mount motor and blower on resilient isolators, if possible.

(e) If possible, reduce the rotational speed of the blower. A 25\% reduction may decrease the total noise output as much as $8 \mathrm{~dB}$. However, consult with a professional heating and air-conditioning engineer for proper advice and instructions, especially if rewiring of motor connections is required.

(f) Install acoustical duct liner on the interior surfaces of the motor-blower enclosure.

Mounting of Equipment:

(a) Place vibration isolators under both the motor-blower and the heating and air-conditioning equipment. Sufficient isolation may be obtained by mounting all the equipment on a 5/8" (16 mm) thick plywood base resting on a $5 / 8^{\prime \prime}(16 \mathrm{~mm})$ thick pad of glass fiber.

A $2-1 / 2^{\prime \prime}$ (6 cm) square, 5/8" (16 mm) thick pad of ribbed rubber or neoprene covered with a hardboard or metal plate placed under each corner of the equipment would also provide adequate isolation. (b) Insert flexible canvas or plastic connectors at the blower end of both the supply and return ducts.

The above measures should reduce the noise output significantly.

Central Return Air Duct:

(a) All interior surfaces of the return air duct should be treated with acoustical duct lining, or

(b) the duct should be fitted with a suitable prefabricated silencer.

Construction of Central Closet: (see Fig. 4-22)

(a) Replace the louvered door with a solid core door equipped with a perimeter rubber gasket and a threshold drop closure.

Caution: The door louvers provided the ventilation of the closet for proper heating and combustion. It is necessary that the ventilation be maintained by other means. See next item.

(b) If the wall studs are exposed on the inside of the closet, build or install an acoustically lined air transfer grille between the studding, as illustrated in Fig. 4-12, to preserve the required ventilation and noise isolation.

(c) Nail resilient channels horizontally to the studding; space channels $24^{\prime \prime}(60 \mathrm{~cm})$ apart; fill space between the channels and the backwall with glass fiber blanket; attach 1/2" (13 mm) thick gypsum wallboard to the channels with self tapping screws.

(d) Install a ceiling of construction similar to item (c) in the closet.

(e) Apply acoustical tile to both ceiling and wall surfaces in 


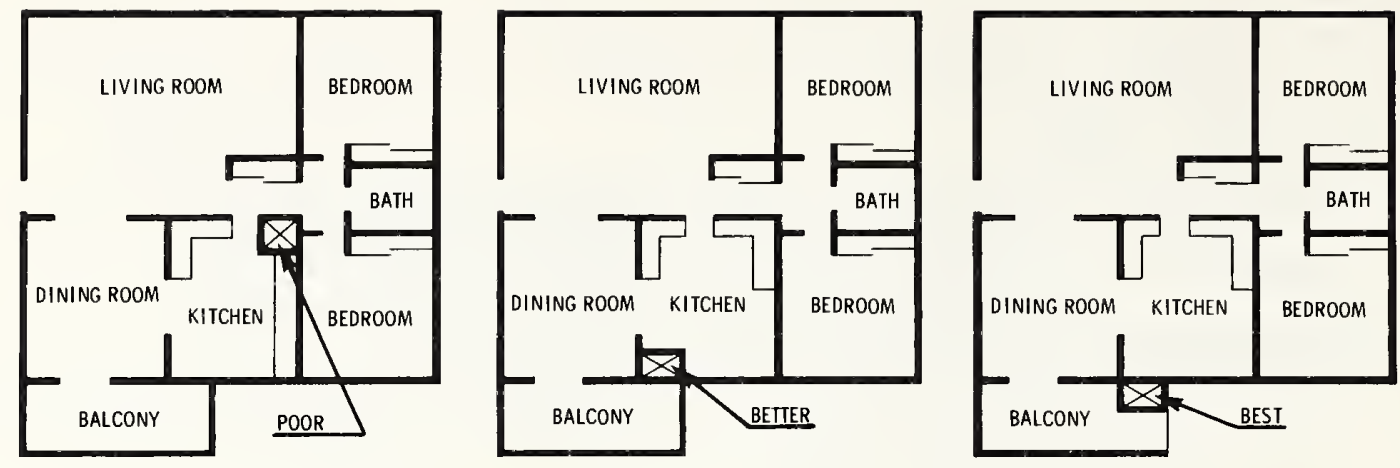

SELECTING PROPER LOCATION FOR CLOSET-INSTALLED FURNACE UNIT
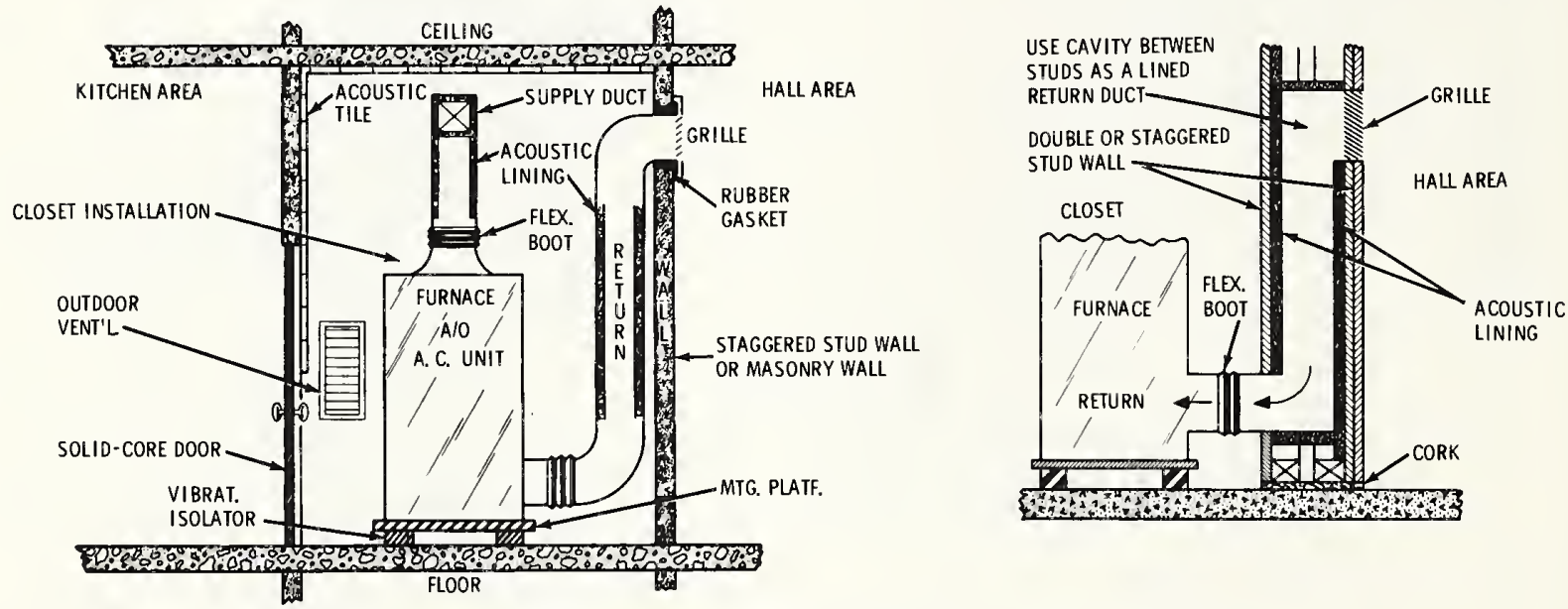

RECOMMENDED DESIGN OF CLOSET AND INSTALLATIGN OF FURNACE UNIT Fig. 4-22. TECHNIQUES FOR THE CONTROL OF NOISE FROM CLOSET-INSTALLED FURNACE
AND AIR-CONDITIONING UNITS

the closet to reduce noise buildup.

CONTROL OF DUCT NOISE: (see Figs. 4-23 and 4-24)

Because metal ducts are extremely efficient transmission paths of airborne noise and vibration, considerable attention must be given to the proper design, construction and installation of duct networks.

\section{Equipment Noise}

Airborne noises such as motor hum, blower-blade noise, bearing or pulley noise, furnace ignition, flame noise, and the creaking and snapping noise due to the expansion and contraction of metal parts are easily transmitted through the ducts to all areas of the house.

If any of these noises are a source of annoyance, you can reduce the level of noise output substantially by installing sound absorbing lining material or a prefabricated silencer in the ductwork. Such treatment is most effective when it is installed in both supply and return branch ducts serving the individual rooms, and preferably near the open or grille end of the duct. The installation of a ten-foot length of duct liner, as measured from the grille or diffuser, may reduce the loudness of the noise as much as $50 \%$ or $10 \mathrm{~dB}$. 

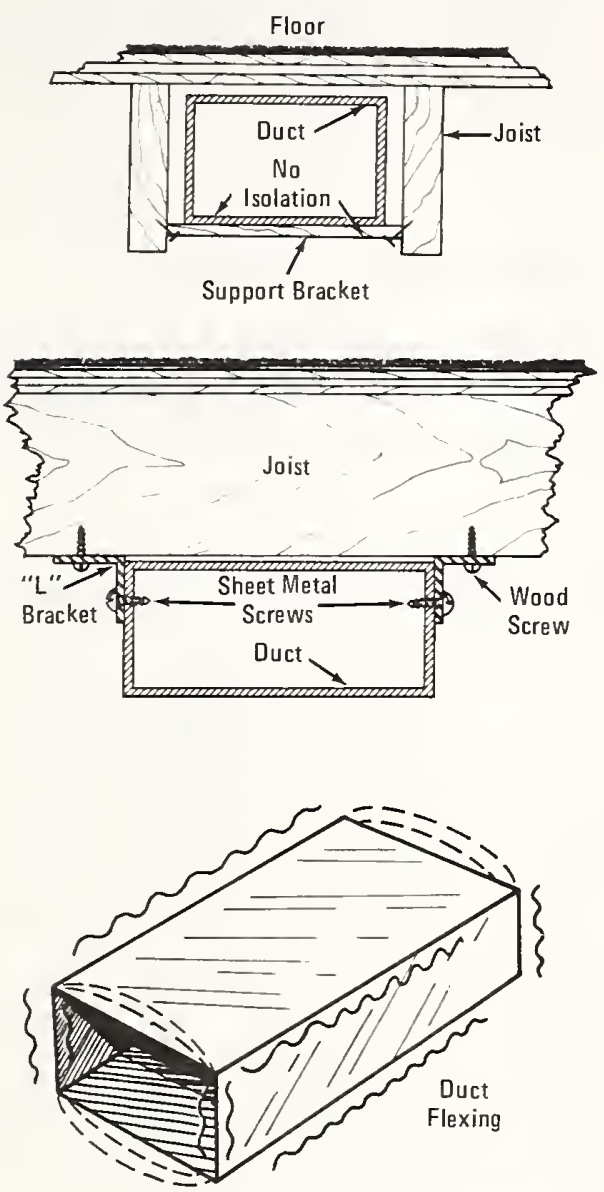
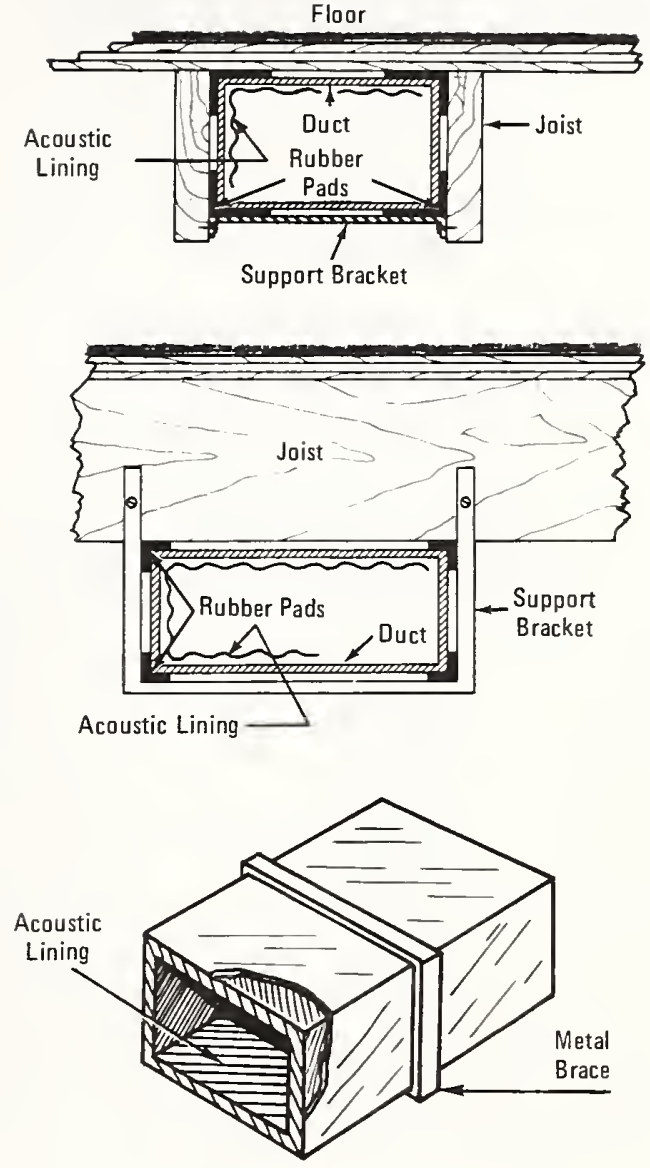

Fig. 4-23. METHODS OF REDUCING NOISE DUE TO AIR FLOW AND VIBRATION IN DUCT SYSTEMS

\section{Cross Talk}

Metal ducts act like speaking tubes on a ship. Noise from one room may be carried to another room through an unlined duct serving both rooms. For example, the problem of "Cross Talk" frequently occurs in a common return duct serving back-to-back bathroom installations, or in common ducts in which the grille openings serving separate rooms or apartment units are too closely spaced. Such problems also arise in exposed, thin-wall main ducts which span across adjacent apartment units, even though such ducts might not have any grille openings. Household noises may penetrate the thin walls of the ducts in one apartment, travel through the short duct passage through the party wall and emerge from the thin-wall duct in the adjoining apartment. Noise transmission from one room to another may also occur by way of openings and holes around poorly sealed duct penetrations through wall and floor structures.

Such noise transmission paths by-pass the sound insulating effectiveness of the intervening party walls and floor structures.

Noise transmitted through ducts, pipe chases, electrical channels, or other passages can be reduced effectively by lining the inside surfaces of such passageways with sound absorbing materials. In typical duct installations, it is possible to obtain noise reductions for high-pitched sounds of the order of 2 to 3 $d B$ per linear foot for $I^{\prime \prime}(2.5 \mathrm{~cm})$ thick acoustical lining. (In metric units, the reduction is about $10 \mathrm{~dB} / \mathrm{m}$ for an acoustical 
NOT THIS
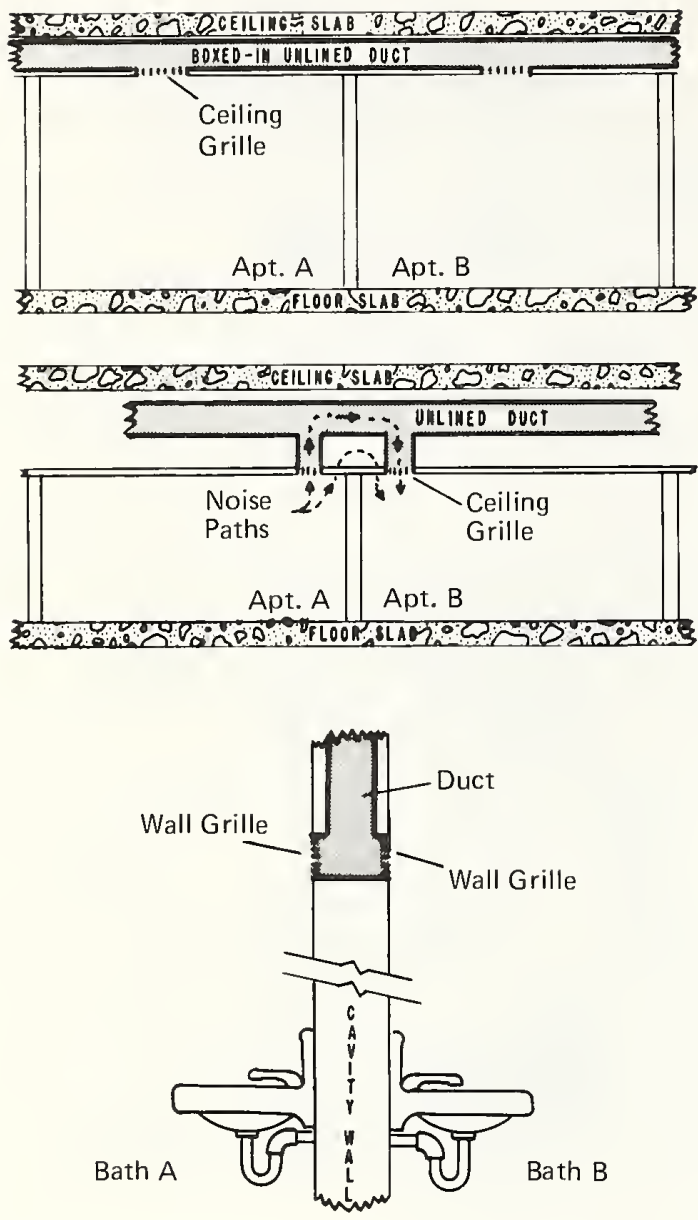

THIS
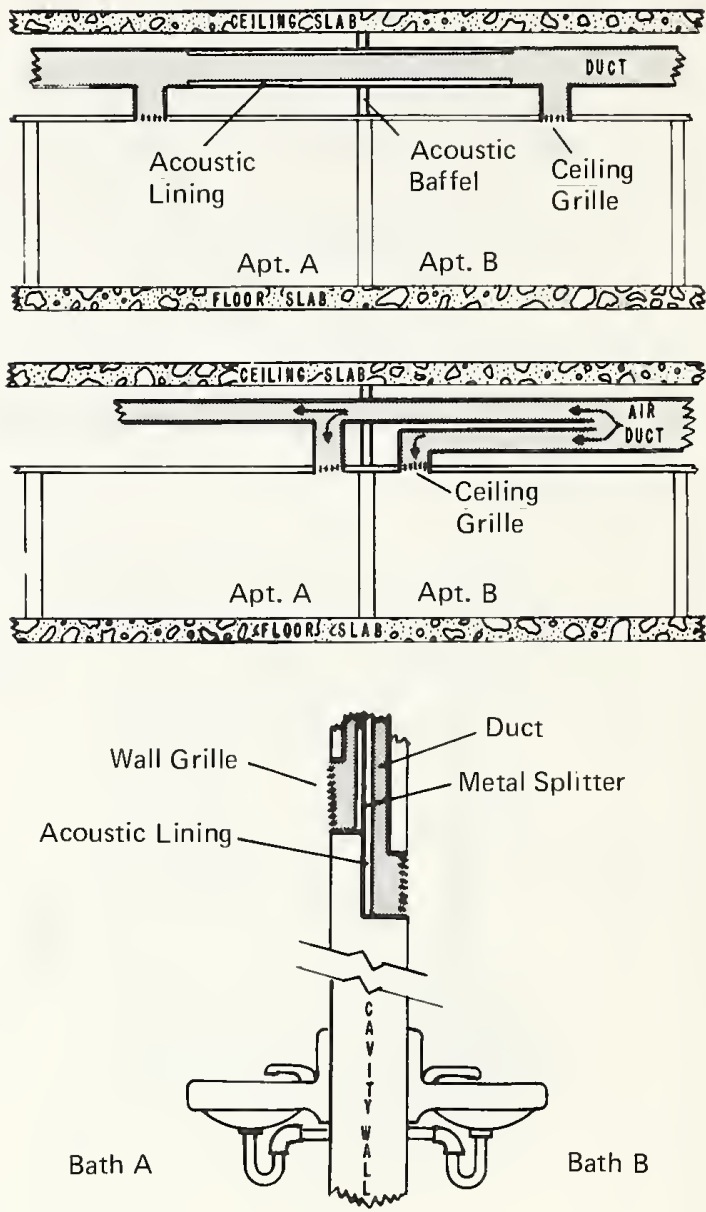

Fig. 4-24. METHODS OF CONTROLLING CROSS TALK AND THE TRANSMISSION OF FLANKING NOISE IN DUCTS

lining $2.5 \mathrm{~cm}$ thick.) A comparable degree of noise reduction for the low-pitched sounds is considerably more difficult to achieve because it usually requires at least a doubling of the thickness and/or length of acoustical treatment. Various types of duct lining and silencers are illustrated in Fig. $3-16$.

If you are disturbed by noises from an adjoining room or apartment that seem to be coming through a ventilation grille or the walls of the duct in your room, try one or more of the following remedies to alleviate the problem.

(a) Line the intervening duct run with acoustical material or install a sound silencer, preferably at the wall junction. (b) Remove existing grille, seal opening, and reinstall grille in the duct at a location as far as possible from the party wall.

(c) Seal all openings where ducts penetrate through the wall.

(d) If the noise is coming through the duct wall enclose the duct in a box made of gypsum wallboard.

\section{Duct Noises}

The creaking and snapping noises caused by the expansion and contraction of ductwork due to temperature variation often are a source of disturbance.

Likewise the booming noise caused by 
the ballooning and collapsing of thin-wall ductwork as the blower cycles on and off may be loud enough to interfere with a person's sleep.

If you are troubled by noisy ducts, the following measures may be useful in reducing or controlling the noise:

(a) Support ducts on resilient mounts or hangers to prevent wall and floor structures from amplifying the noise. The resilient mounting will permit the ducts to expand and contract without binding against some wall or floor construction.

(b) Wrap the ductwork with two or three layers of building paper which will act as a vibration damper. The paper may be taped in place or held with wire ties. This treatment will tend to suppress any vibration set up in the duct walls and restrain the "ballooning" action of the duct by adding some much needed mass and bracing to the flimsy duct work.

(c) Install 3/4" (19 mm) thick glass-fiber board sound absorbing lining on the inside surfaces of the ducts where "ballooning" occurs. The glass-fiber board, which is rigid enough to support the duct walls, and to act as a vibration damper, will also absorb some of the noises generated within the duct system.

(d) Using sheet-metal screws, install braces made of metal bars or folded strips of sheet metal across the largest or widest sections of ductwork or at places wherever "ballooning" occurs. The braces will provide adequate support of the duct walls and thus prevent this type of motion.

\section{Air Flow Noise}

High velocity air flow generates noise not only within the ducts but at the face of the outlet grille or diffuser as well. The intensity of flow noise is strongly velocity dependent. For a given duct area, decreasing the flow velocity $50 \%$ may decrease the output noise level as much as 15 to $24 \mathrm{~dB}$.

Air flow velocities of about 15 feet
$(4.5 \mathrm{~m})$ per second are quite acceptable for most homes and apartments. However, to be on the safe side, flow velocities in noise sensitive areas should be lowered to about 8 or 9 feet $(2.5-3 \mathrm{~m})$ per second.

The most expedient way of reducing air flow velocity is to decrease the rotational speed of the blower, providing that it does not interfere with the heating or cooling efficiency of the system.

\section{Grille Noise}

Noises generated within induction units or at the faces of outlet grilles or diffusers give rise to numerous complaints from tenants. The intensity and characteristics of such noise are dependent upon the air flow velocity and the size and design of the outlet units. For example, high velocity air striking the face of an outlet grille often generates a high-pitch whistling noise.

Removal of the grille is a simple expedient test to determine the cause of noise. If there is no appreciable reduction in the noise output without the grille, the noise may be due to turbulence within the discharge duct or some other source farther back in the duct system. If there is a marked reduction in the noise output without the grille, it is evident that the grille was at fault and therefore should be replaced with one of better design, or the air flow speed should be reduced if practicable.

The following points are worth remembering when choosing outlet grilles and diffusers for quiet operation.

(a) Grilles or diffusers which radiate little noise are made of heavy-gage metal with widely-spaced streamlined deflectors devoid of any sharp corners or edges.

(b) A low-spread air diffuser will generate substantially less noise than a large spread unit, all other factors being equal.

(c) Grilles constructed of wire mesh or perforated metal facings with large openings are less noisy than those with tightly woven or small openings which restrict the air flow. 
There are two types of noise associated with furnace combustion that sometimes cause annoyance. They are the muffled explosion noise when the gas or oil is ignited and the very high-pitched whistling noise caused by high-velocity gas flow through the fuel nozzle or burner jets.

The most expedient way of dealing with this problem is to have a heating engineer adjust the fuel pressure or install nozzles with proper openings for quiet operation.

If the furnace operation is still too noisy following such adjustments, the installation of sound absorbing material in the ductwork will reduce the noise to acceptable levels.

\section{Steam Heat Noise}

The hammering and knocking noises in steam pipes and radiators may result from water being trapped in the lines. This frequently is caused by vapor lock, partially open valves, or improper drainage of pipes and radiators.

If you have noisy radiators, you may alleviate the problem by:

(a) Opening all valves completely to allow trapped water in the system to drain back to the boiler.

(b) Placing thin blocks or wedges under the legs of the radiator opposite the input end to increase the drainage angle, and thus allow any trapped water to drain away.

(c) Replacing faulty pressure release valves.

(d) Inserting heat-resistant resilient material around the pipes at points of support or penetrations through walls or floor structures, to eliminate the rubbing contact and the resulting noise caused by the expansion and contraction of the pipes.

\section{Noise from Baseboard Heating Systems}

Homeowners and apartment dwellers alike frequently complain about the noisy operation of baseboard heating systems, in particular the hot water heating system and the electrical heating system.
Causes of Noise

The noise produced by such heaters generally is caused by:

(a) the expansion and contraction of pipes, heater components and housing,

(b) water flow and turbulence, and

(c) pump and motor vibration.

\section{Remedies}

(a) Heaters and associated piping generally creak at joints, connections and points of support or contact with building structures during expansion and contraction due to temperature variation. When the units are rigidly connected to walls and floors, these structures behave like sounding boards and amplify the noise. Therefore, in order to minimize this problem, heat-resistant resilient sleeves or collars should be placed at all pipe clamps, supports and penetrations through wall and floor structures. Similar measures should be taken to isolate the heater units from the walls and floors using heat-resistant resilient spacers and leg mounts.

Reducing the expansion and contraction noise of the heater assembly itself is somewhat more difficult to achieve. Sometimes, attaching braces of folded sheet-metal strips to the long narrow panels of the heater assembly will suppress the noise due to buckling and popping of the panels during the heating cycle.

Securely fasten all loose panels and hardware on the heater assembly to suppress buzzing and rattling noises.

(b) If the pipes tend to sing a high-pitched tone, this is an indication that there is high-velocity, turbulent flow in the system, which generally is caused by using an oversize, highspeed pump and undersize piping. 
This problem can best be alleviated by decreasing the rotational speed of the pump or replacing it with a slow-speed model.

(c) Pumps and drive motors should be mounted on resilient pads to prevent vibrational excitation of the floor.

Flexible hoses similar to auto heater or radiator hoses should be installed in a U-shaped configuration between the pump and pipe system to suppress the transmission of pump vibration to the pipe system.

Installing a surge tank in the system at the discharge end of the flexible hose will further suppress vibrations from the pump and dissipate pulsation from the impeller.

\section{Electrical Baseboard Heaters}

\section{Causes of Noise}

(a) The major source of noise is the expansion and contraction of the heater assembly and component parts and the amplification of the noise by wal1 and floor structures in direct contact with the heating system.

(b) Another source of annoyance is a low frequency resonance of the heater assembly, which sounds like the low-pitched hum of a transformer.

(c) Some insta1lations have individual thermostat controls in each room. The clicking of a thermostat in the still of the night can be extremely irritating, particularly if a person is trying to sleep.

\section{Remedies}

(a) To alleviate the noise of expansion and contraction of the heater assembly, use the method discussed above for hot water heaters.

(b) In a typical installation, the low-pitched humming noise genera $11 y$ is radiated at amplified levels by a large wall which is directly connected to the resonating heater assembly. Structurally isolating the heater from the floor and wall with heat-resistant, resilient spacers and leg mounts should reduce the noise output to acceptable levels.

Attaching braces made of folded sheet-metal strips to the long narrow panels of the heater assembly will further suppress the low-pitched huming noise or resonance.

(c) If your thermostat is excessively noisy, remove the cover plate to determine what type of unit was installed. The noisy thermostats generally use bi-metal, electromagnetic- assisted contact elements which operate off high or line voltage. The "Silent" thermostats use mercury-filled tubes as the contact elements and operate off a low voltage supply.

Consult with your heating engineer for advice regarding replacement or interchange of such units.

There are a number of methods that may be used to reduce the clicking noise of thermostats. However power must be shut off before the following methods can be tried.

(1) Remove the cover plate and remove the thermostat from the electrical box. Reinstall the unit with rubber grommets or other resilient mounts to isolate it from the box and wall structure. Replace the cover plate.

(2) If additional noise reduction is desired, wrap the outside surfaces of the electrical box with a thin layer of lead or metal-foil tape to damp the vibration. Place lead or tape on the back of the cover plate.

\section{CONTROL OF PLUMBING NOISE}

Most homeowners and apartment dwellers readily admit that noise arising from the use of plumbing facilities anywhere within the home generally can be heard throughout 
the dwelling, and frequently in adjacent dwelling units as well. Probably because of this characteristic invasion of a person's privacy, plumbing noise ranks not only as an irritating disturbance but as the most offensive noise to which building occupants are exposed.

In any case, it is extremely difficult to isolate yourself from plumbing noise or to prevent it from disturbing other occupants in the building.

Causes of Plumbing Noise and

Methods of Control

Water pipes and fixtures are rather ineffective noise radiators because of their small radiating areas. The major problem arises when such sources are rigidly coupled to large efficient noise radiating surfaces such as wall, ceiling and floor structures. Such surfaces, acting as sounding boards, radiate the noise at amplified levels.

Techniques for the reduction and control of common types of plumbing noises are given below. They are listed in preferential order and should be combined for greatest effectiveness.

\section{Isolation of Plumbing System}

Unfortunately, most of the plumbing system is installed within the walls and is thus inaccessible to the home owner or apartment dweller. He, therefore, is somewhat restrained in using the most effective measure to remedy plumbing noise problems, that is to vibration isolate the piping from all large wall and floor surfaces which act as sounding boards. However, he should make every attempt to isolate exposed piping with bands or sleeves of rubber, neoprene or glass fiber at al1 points of support, including pipe clamps, straps, and penetrations through wall and floor structures. If necessary, oversized clamps should be used to accommodate the thickness of the resilient sleeve.

\section{Expansion and Contraction of Pipes}

The expansion and contraction of pipes produce a staccato-like series of creaking, squeaking and snapping noises which are caused by the sliding or binding of the pipes against studding or other supports. Isolating the pipes from building structures as described above will alleviate the problem.
Noise from Turbulence and High-velocity Water Flow

High water pressures with resultant high flow velocities cause turbulence particularly around bends, valves, taps and connectors which usually contain many sharp edges and constrictions. The familiar hissing noise, that frequently occurs at partially opened taps, is associated with turbulence. It has been suggested that this noise is due to the combined action of eddies and collapsing water vapor bubbles.

Although turbulent flow is considered to be the chief cause of plumbing noise, the onset of cavitation in a plumbing system will result in much higher noise levels. Both conditions may exist simultaneously, especially around constriction in high pressure systems. Cavitation is associated with the collapse of vapor bubbles, which are formed at the constriction by a critical combination of high velocity and low pressure.

High velocity flow in a plumbing system, due chiefly to undersized piping, gives rise to turbulence which frequently generates excessive noise. A noticeable reduction in noise level may be obtained by using proper size piping to lower the water velocity. Flow velocities of the order of 6 $\mathrm{ft} / \mathrm{sec}(2 \mathrm{~m} / \mathrm{sec})$ or 1 ess in domestic systems have been found to be quite acceptable. Specified flow capacity requirements can be met and a substantial reduction in noise can be obtained by using both pressure regulators and larger diameter piping in the plumbing system.

High-pressure plumbing systems are inherently noisy, due to the resultant turbulent flow generated within such systems. The static pressure of main water-supply lines of buildings with three stories or less should be regulated so that it will not exceed 50 psi (345 kilopascals). The water pressure in branch lines serving individual apartment units should not exceed 35 psi (241 kilopascals). In high-rise structures where high-pressure main supply lines are required, pressure reducers or regulators should be used in supply branches at various floors to maintain water pressure within the above limits.

Pipe Hammering, Chattering and Vibration

The noisy hammering of a plumbing system is usually caused by the sudden 
interruption of water flow; for example, by a quick-closing tap. The sharp pressure build up at the point of interruption forms a shock wave which reflects back and forth in the system. The multiple reflections produce a series of hammer-like noises which gradually decrease in loudness as the energy of the shock wave is dissipated. The sudden release of pressure by a quick-opening valve which discharges into a section of piping with a narrow constriction, elbow or tee connector also may cause hammering of the plumbing system.

Hammering can also occur when pipes are loosely supported on V-shaped wire hangers. Water surges or pulsations in the pipes may cause them to bounce or vibrate in the hangers, or perhaps to swing and strike against other parts of the building. Replace the wire hangers with pipe clamps. Inserting a rubber sleeve around the pipe at the clamp and fastening it securely to some solid structure will remedy the problem.

Defective, loose or worn valve stems, faucet washers or seals give rise to intense chattering of the plumbing system. The defective device frequently can be pinpointed without difficulty, since immediate use of the device causes the vibration which generally occurs at some low flow velocity setting and diminishes or disappears at a higher flow setting. For example, if vibration occurs when a particular faucet or tap is opened partially and diminishes when it is fully opened, the faucet more than likely has some loose or defective parts.

Pipe vibration frequently is caused by motor driven pumps which, by virtue of rigid mechanical coupling, transfer their vibrational energy to the piping system. Noises due to such sources are easily recognized, since they consist mostly of pure tones associated with the rotational speed of the pumps or motors. The current trend toward using miniaturized, high-speed, shaft-coupled motor pumps has intensified this problem.

The above pipe noise problems generally can be alleviated by using flexible connectors and air chambers in the piping system and by replacing worn or defective valves and faucet washers. Pitted valve and washer seats also should be refinished or replaced.

Flexible connectors should be used in coupling supply and drain pipes to vibrating appliances such as pumps, garbage disposers, clothes and dishwashers. Since such appliances frequently have electrically operated shut-off valves, air chambers or other shock absorbing devices should be installed in supply and drain lines to prevent water hammering of the plumbing system. The air pockets, rubber inserts or spring elements in such devices act as shuck-absorbing cushions.

Noise from Draining and Dripping Water

The draining of water from bath tubs, basins and toilets produces gurgling noises which frequently are more annoying than those associated with the filling of such units. The noise problem is intensified when vertical drain systems do not run directly to the basement, but branch off into horizontal pipe runs which usually are supported from floor joists. Falling water striking the horizontal piping sets the drain system into vibration which in turn is transmitted to the building structure.

The noise of splashing of water such as that associated with filling a bath tub can be irritating. Attaching a long resilient hose to the faucet which will reach the bottom of the tub is an effective way of eliminating this noise problem. In the case where the noise of a dripping faucet is a source of annoyance in the still of the night, as a temporary solution, attaching a string or draping a wash cloth from the faucet will channel the water drops to the drain without noise. of course, replacing the worn washer seal would be a more permanent solution.

\section{Noise Due to Entrapped Air in Pipes}

A relatively common noise problem that is generally confined to newly constructed buildings is caused by entrapped pockets of air in the plumbing systems. The combined action of water pressure and compression of the air pockets may produce intense noise and vibration disturbances which are characterized by explosive bursts, spewing and spitting of water and air from open faucets or taps and hammering or knocking of the piping system. Such problems seldom are a source of complaints, unless they persist for extended periods. Generally speaking, the problem eventually corrects itself by gradual release of the entrapped air through continued use of the plumbing services.

\section{HOW TO DESIGN A QUIET PLUMBING SYSTEM}

If you are planning to build a home with a quiet plumbing system, you must 
incorporate the following design elements in the early stages of your building plan.

(a) Vibration Isolate all Plumbing Systems: (see Figs. 4-25 and 4-26)

A typical modern home or apartment may have three separate piping systems; the hot and cold water supply system, the drainage and venting system, and the hydronic piping system, which includes air-conditioning, and steam and hot water heating systems. One of the most effective solutions to pipe noise problems is to keep the pipe isclated with resilient sleeves and hangers from building structures with large radiating surfaces such as walls, ceilings and floors. Pipes in buildings behave like tuning forks using such structures as sounding boards to amplify the noise levels. (b) Use a Simple Plumbing Layout:

A well designed plumbing system with a minimum of fittings and bends is substantially less noisy than a complicated layout. Proper size fittings and large-radius elbows or bends should be used for quiet performance. Large diameter piping should be used for all main supply lines. Separate branch lines of smaller diameter should be installed to serve each individual area where required.

(c) Insta11 Pipes Away from Quiet Areas:

Pipes should not be installed in any walls enclosing bedrooms, living rooms, studies or other rooms where privacy and quiet surroundings are essential. For example, in order to prevent the transmission of noise into bedroom
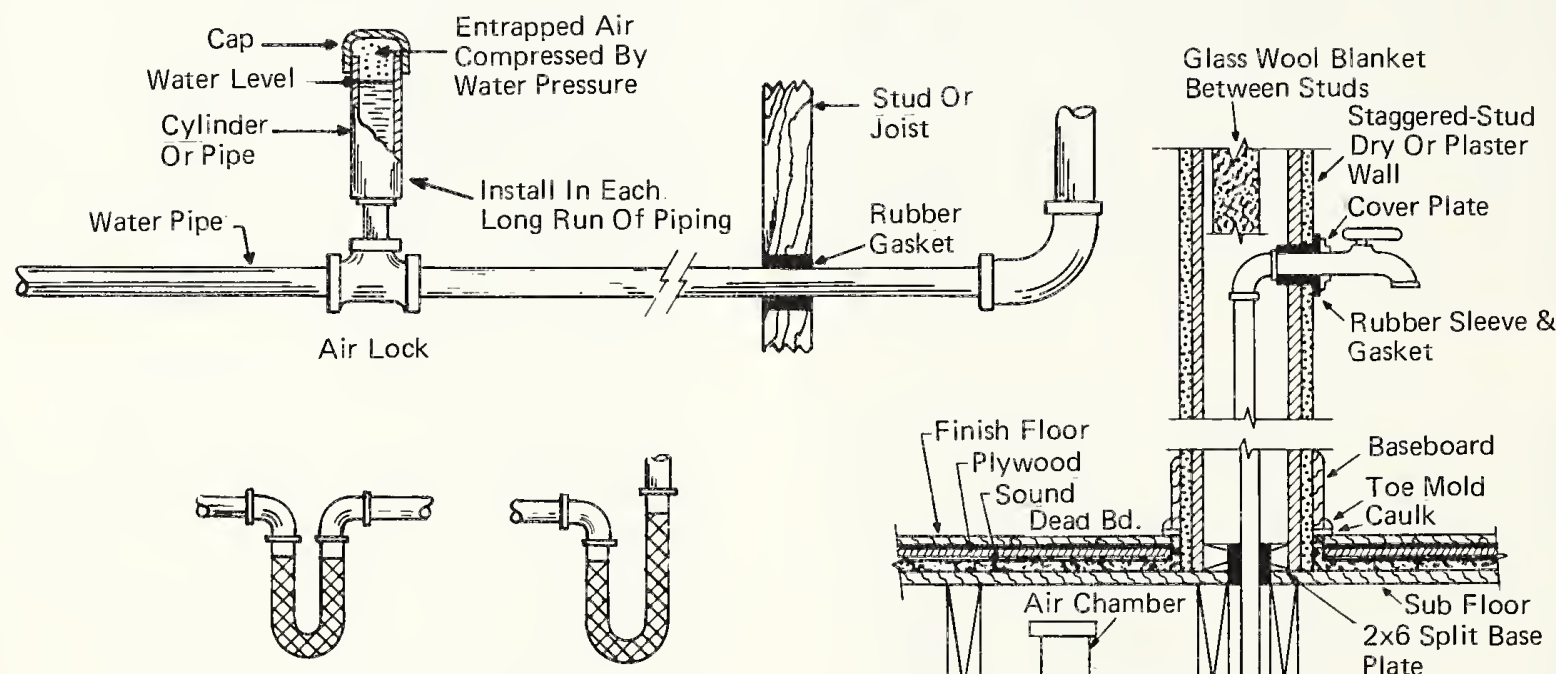

PROPER HOOKUP OF FLEXIBLE CONNECTORS
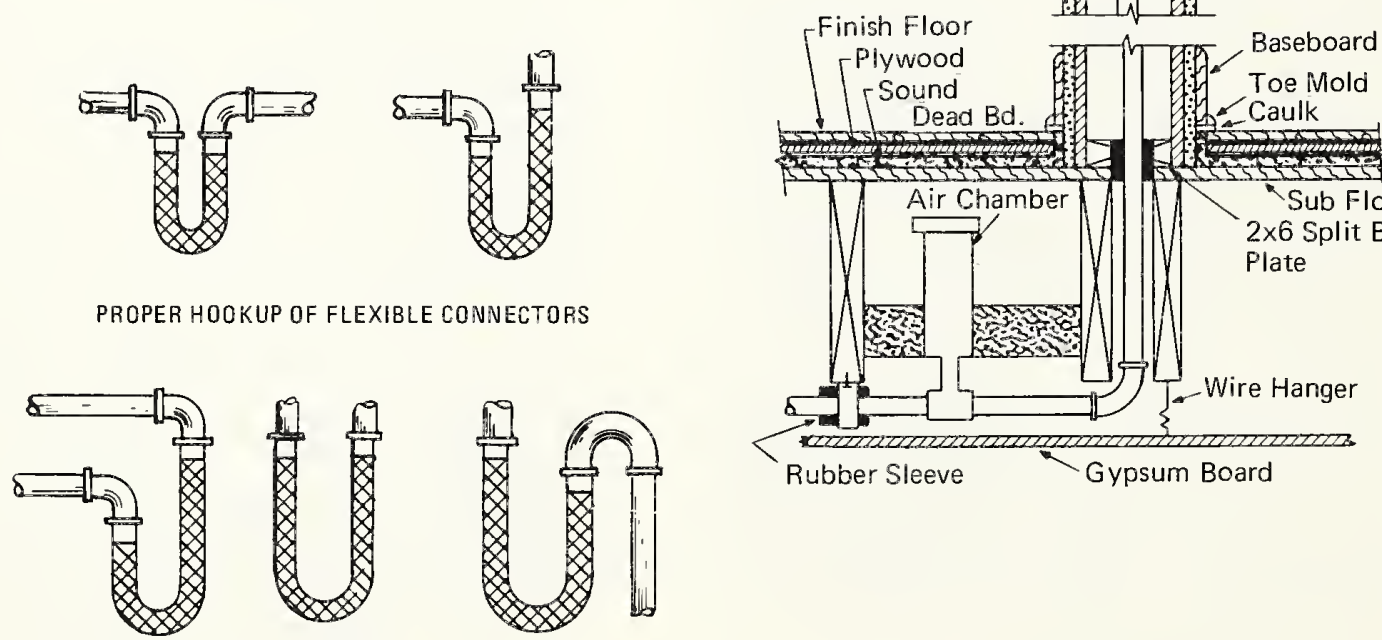

Fig. 4-25. TECHNIQUES FOR CONTROLLING NOISE IN PLUMBING SYSTEMS 

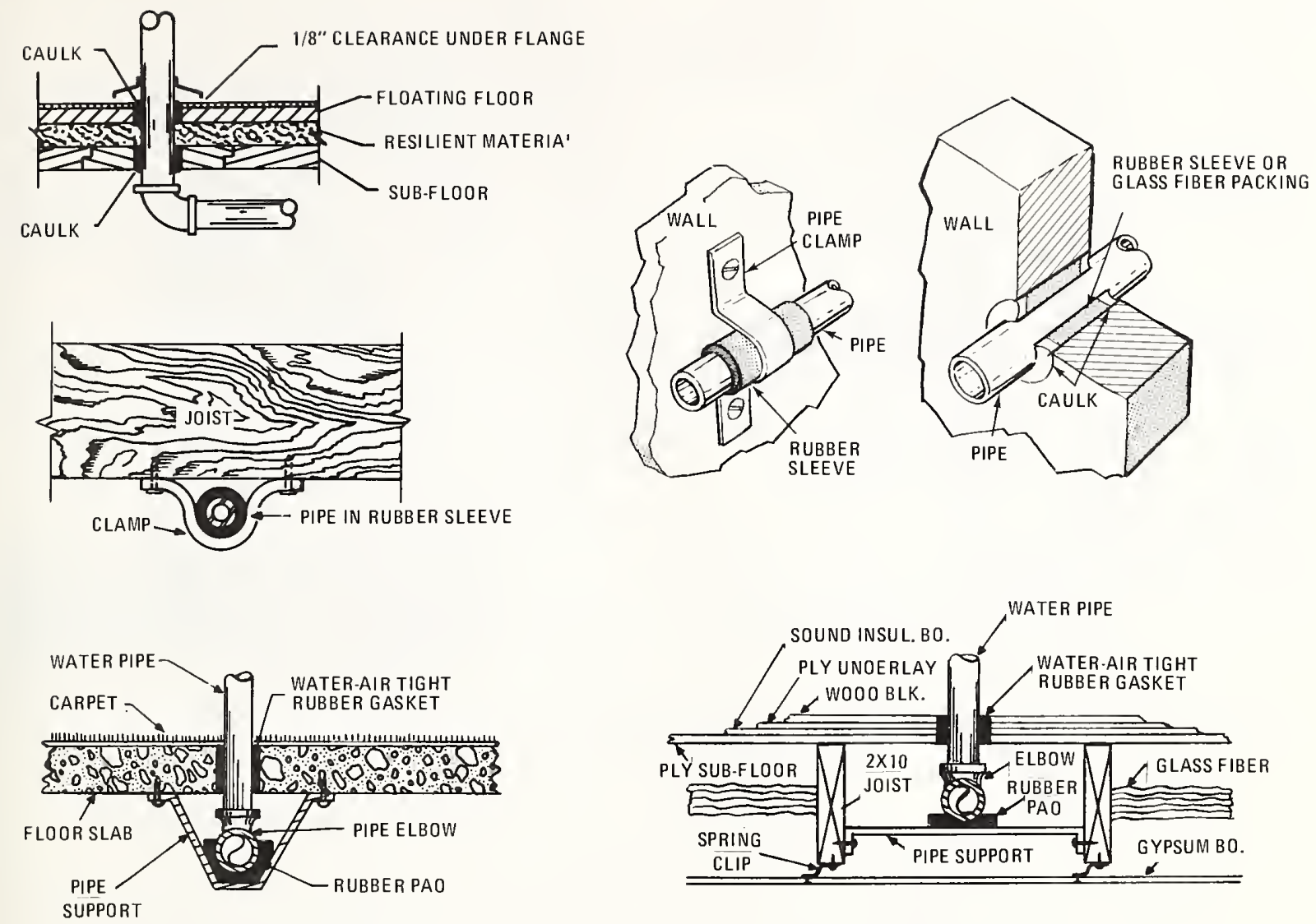

Fig. 4-26. METHODS OF INSTALLING PIPES FOR QUIET OPERATION

areas, supply and drain pipes should not be installed in walls separating bathrooms and bedrooms. Piping should be installed in partition walls which separate adjacent bathroom or kitchen areas. Supply and drain pipes must be isolated from internal studding or wall surfaces.

(d) Install Quiet Fixtures:

Siphon-jet toilet and flush tank fixtures with adjustable flow valves are considerably less noisy than conventional models. Taps and faucets using full-ported nozzles and equipped with anti-splash or aeration devices produce little noise.

(e) Install Bathroom Fixtures on Resilient Bases and Mounts:

(See Figs. 4-27 and 4-28)
Bath tubs, toilets and shower stalls should be set on underlayments of cork, rubber, neoprene or other resilient materials or installed on floating floors to reduce the transmission of noise due to falling water. Likewise, the fixtures should be vibration isolated from supporting walls by means of resilient gaskets. Such mounting precautions should be observed with respect to installation of wash basins and faucet fixtures as well.

(f) Seal Air Leaks Around Pipe Penetrations:

To prevent noise leaks, seal all openings around pipe penetrations through wall and floor structures with a non-setting waterproof caulking compound. Party walls between bathrooms should be 
Apt. A Apt. B
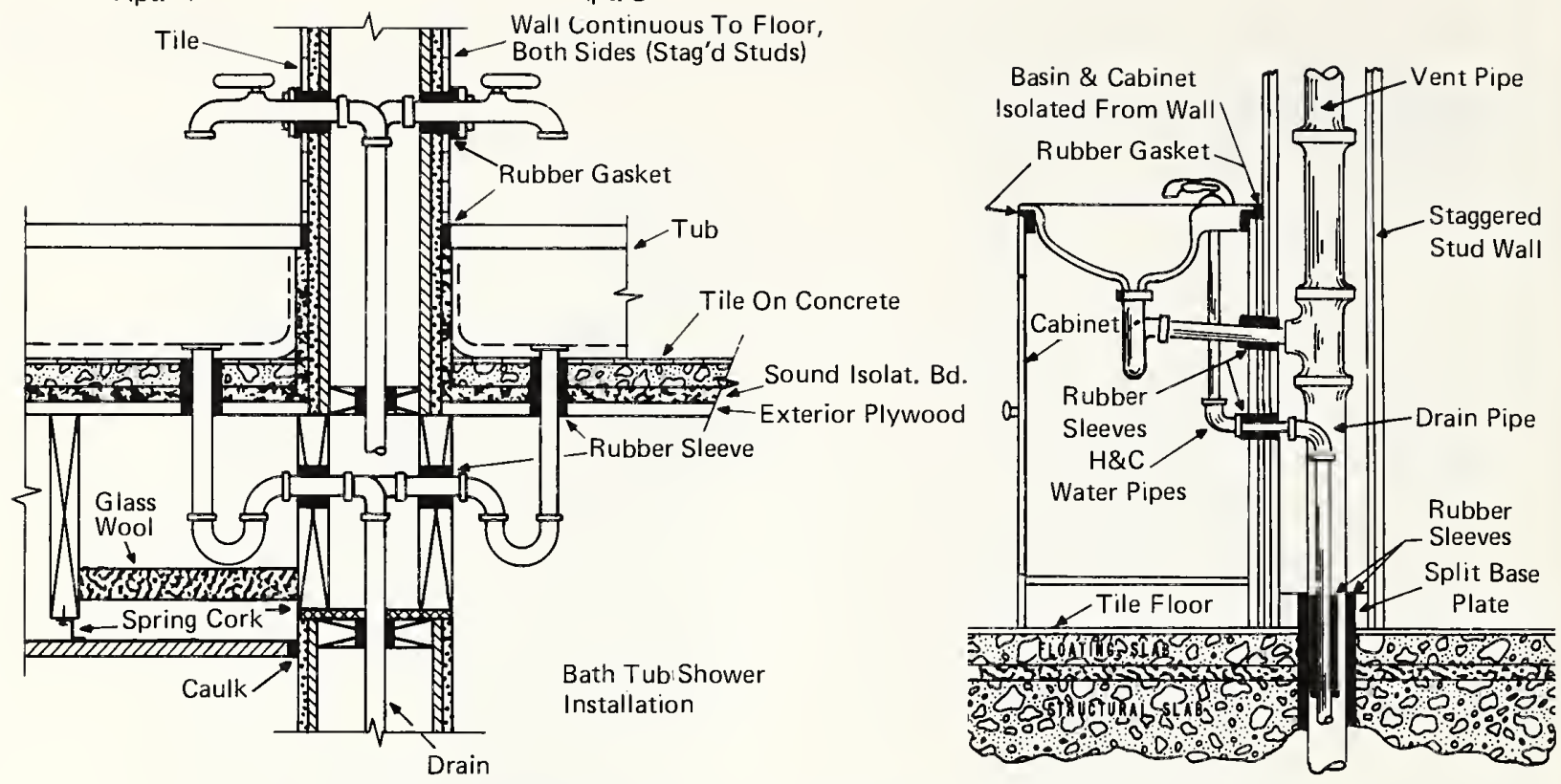

Fig. 4-27. PROPER INSTALLATION OF BATH TUBS AND WASH BASINS

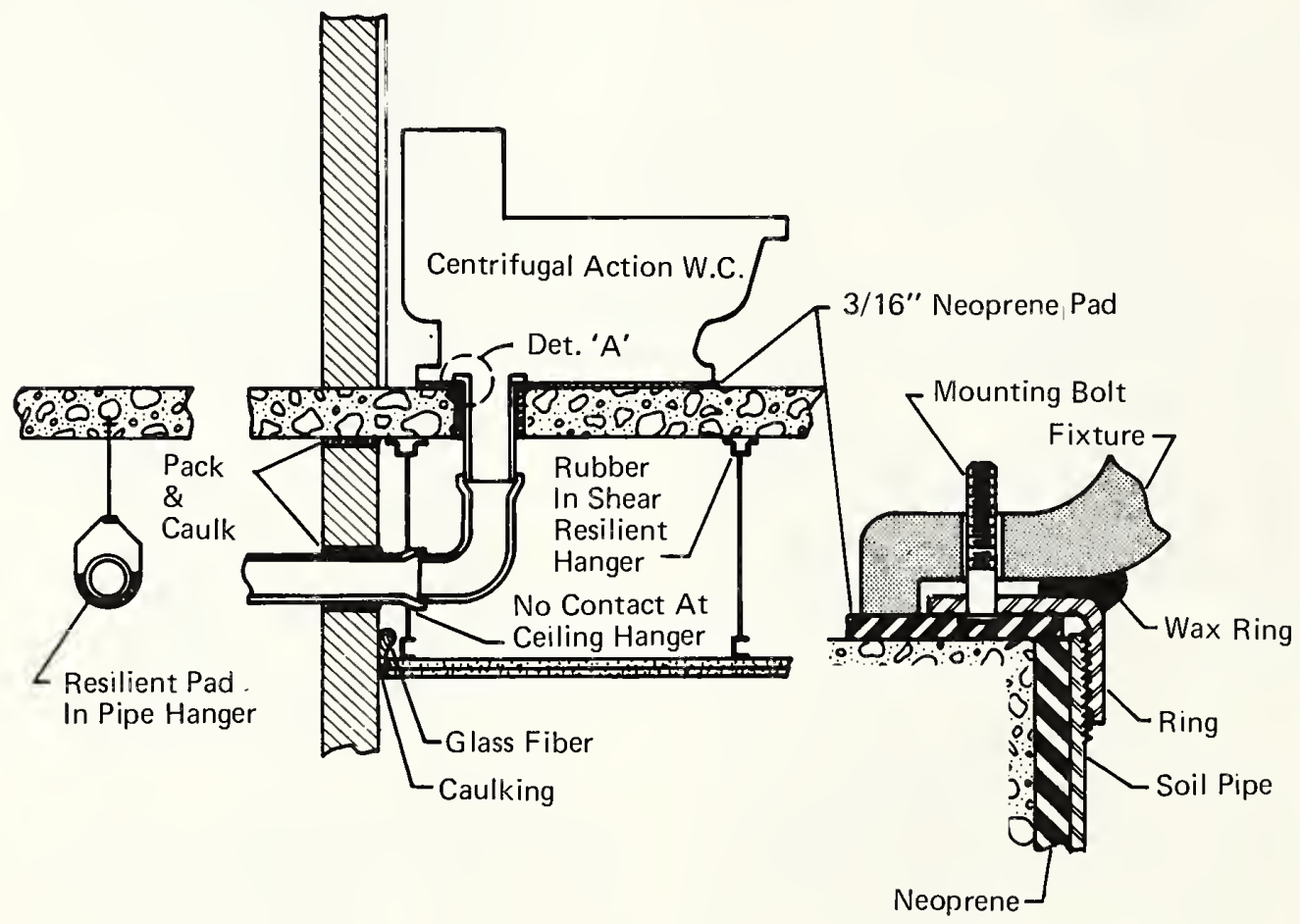

Detail ' $A$ '

Fig. 4-28. PROPER INSTALLATION OF A TOILET FOR QUIET OPERATION 
completely finished to the floor level on both sides, particularly in back-to-back tub and/or shower installations. Failure to surface the walls behind tubs results in serious noise transmission problems. Likewise, both subflooring and finish flooring in bathroom areas should be completely finished before tubs and shower stalls are installed.

(g) Enclose Large Pipes:

Large diameter pipes in high pressure systems frequently radiate considerable noise, especially when they are exposed. Such pipes should be boxed in gypsum board enclosures, preferably lined with acoustical material. An alternate, though somewhat less effective, technique is to enclose the pipes in thick glass fiber jackets with heavy impervious outer coverings of plastic or leaded-vinyl materials. It has been suggested that the glass fiber jackets should have a density of about $6 \mathrm{lb} / \mathrm{cu}$ ft $(96$ $\mathrm{kg} / \mathrm{m}^{3}$ ) and a thickness of at least 3 inches $(8 \mathrm{~cm})$. The impervious covering should, weigh at least 1 $1 \mathrm{~b} / \mathrm{sq} \mathrm{ft}\left(5 \mathrm{~kg} / \mathrm{m}^{2}\right)$.

CONTROL OF NOISE FROM RAIN GUTTERS AND SPOUTS

An irritating source of noise, particularly in the still of the night, is the turbulent flow of rainwater in gutters and downspouts during a storm, and the incessant dripping and plunking of the water after the storm is over. Rigid attachment of the downspouts or drain pipes to the exterior walls of bedroom areas is the chief cause of the problem. The interior surfaces of the walls, which perform as sounding boards, tend to reinforce or amplify the drain pipe noise. The following measures should be used to minimize disturbance from such noise.
a. Avoid installing vertical drainpipes outside of bedroom areas.

b. Structurally isolate vertical drains from the building by inserting soft rubber or neoprene sleeves or collars around the drainpipes where they are clamped to the wall.

c. Replace the conventional metal elbow at the base of the drainpipe with a soft rubber boot or plastic hose.

d. When replacing existing rain gutters, select units made of heavy gage metal, with rounded corners, and coated with vibration damping material.

Refer to Fig. 4-29 for proper installation of rain gutters and vertical drainpipes.

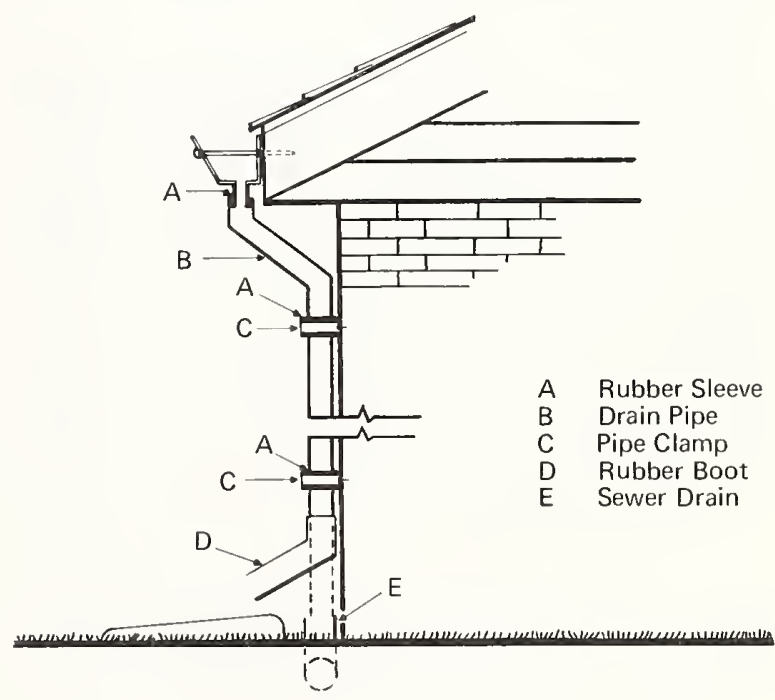

Fig. 4-29. METHODS OF CONTROLLING NOISE FROM RAIN GUTTERS AND DOWNSPOUTS 
As we know from experience, residential areas that formerly were quiet and peaceful in time become engulfed by highly disturbing sources of noise. Changes in zoning ordinances often permit development of noisy industrial parks or shopping centers on the outskirts of residential areas. Ordinary highways eventually become high-speed noisy expressways which invade the peace and tranquility of quiet communities and suburban areas that often are encircled or flanked by the roadway. In some cases, local neighborhood noise caused by traffic, lawn mowers, chain saws, barking dogs, or perhaps children at play may be the most disturbing problem. In other cases, noise from community swimming pools, playgrounds, athletic fields, amusement parks or sport arenas may be the major source of annoyance. The outdoor noise levels generated in neighborhood environments by the various sources cited above might be described as being in the moderate to high level range, or roughly 70 to $95 \mathrm{~dB}$.

However, the greatest number of noise complaints come from home owners and apartment occupants living in residential areas near airports. The development of medium jet airports into international airports handling much larger and noisier aircraft has resulted in more serious noise problems affecting much larger communities and populated areas. The expansion of small private airports to handle the new breed of sma11 business jet aircraft has created similar noise problems in small, formerly quiet communities.

Aircraft noise levels at the location of the home or apartment site will vary depending chiefly on its distance from the airport or flight path, and type and altitude of aircraft. In any event, dwellings near large airports or directly under flight paths may be subjected to rather intense levels of noise ranging from 90 to $110 \mathrm{~dB}$ or more.

We will discuss first the steps for improving the sound insulation of houses in moderately noisy neighborhood environments, and deal separately with the more difficult problem relative to aircraft noise intrusion at the end of this section.

Generally speaking, most single-family houses are an assemblage of light-frame interior partitions enclosed and supported by a light-frame exterior shell. If this shell were continuous and formed an air-tight seal around the enclosed space, it would provide at best a limited amount of sound insulation. Such construction typically has a sound transmission class rating of about STC 30-35. However, this shell usually contains several windows and doors. With their customary air and noise leaks, the windows and doors may reduce the STC rating of the exterior shell of the house to a value as low as STC 20. However, there are ways of preventing this reduction, and of improving the sound insulation of the house.

Reducing the Intrusion of Neighborhood Noise

If you have a home in a noisy neighborhood and are disturbed by the intrusion of outdoor noise, the following recommendations may alleviate the problem.

(1) The first rule of thumb is to install a central heating and air conditioning system in the house. This will eliminate the need to open windows and doors for ventilation purposes and thus make it easier to reduce the intrusion of outdoor noise.

(2) Existing windows should be caulked or equipped with gaskets to provide an air-tight seal.

(3) If the noise source is high-pitched and very directional, install storm windows with caulk or gasket seals on the side of the house facing the source. It is still more beneficial to install storm windows, properly sealed, on a11 existing windows. This will provide additional insulation against low-pitched noise as well as noise approaching from other directions.

(4) Exterior doors should be provided with soft resilient gaskets and threshold seals. This applies to sliding doors as well.

(5) Install storm doors equipped with resilient gaskets and threshold seals. Sliding doors also should 
be provided with storm doors. The use of storm doors, windows and weather stripping will also conserve energy by reducing air leakage and making it easier to maintain constant indoor temperatures.

(6) Seal or caulk all openings or penetrations through walls particularly around water or gas pipes, electrical cables, and refrigerant lines.

(7) Cover mail slots in doors; install hinged cover plates on clothes dryer heat vents, bathroom and kitchen exhaust ducts, and central vacuum system dust discharge chutes.

(8) Close the fireplace damper, install close-fitting cover plates at clean-out openings and erect a 1/2-inch (13 mm) thick removable, plywood barrier in front of the fireplace opening.

Adoption of the above measures would improve the sound insulation of the exterior shell of the house about $6 \mathrm{~dB}$ for low-pitched sound and more than $10 \mathrm{~dB}$ for high-pitched sounds; these measures would reduce the louaness of the intruding noise by about 25 to 30 percent, respectively.

\section{Reducing the Intrusion of Traffic Noise}

Homes or apartments of conventional construction that are located very near to heavily traveled roadways or expressways are particularly vulnerable to the intrusion of excessively high levels of noise. Since the soundproofing of existing dwellings may be costly and impractical, the occupants should choose those solutions which provide the most noise reduction at least cost. In addition to the recommendations described above, the homeowner should incorporate, on a step-by-step basis, the following noise control measures, until the noise has been reduced to a tolerable level.

(1) In existing windows, install glass panes of double thickness on the side of the dwelling facing the noise source.

(2) Install storm windows over the same windows. Select storm windows having glass panes mounted or encased in rubber gaskets.
(3) Line perimeter surfaces between the existing windows and storm windows with acoustic tile or sound absorbent lining.

(4) Replace existing hollow-core entrance doors with solid-core, soundproof doors provided with perimeter gaskets and threshold seals (see Fig. 4-7).

(5) If intruding traffic noise disturbs your sleep and your bedroom faces the roadway, choosing a bedroom which is located on the opposite side of the house may reduce the level of intruding noise about $10 \mathrm{~dB}$. Selecting a bedroom on the side of the house that is partially shielded from the noise source should reduce the level of the intruding noise about $6 \mathrm{~dB}$.

(6) Install a barrier wall or fence between your house and the roadway. To be effective, the fence should have a solid, continuous surface without any openings or holes. The fence should be relatively long and tall enough to shield, or hide, the entire roadway when viewed from the nearest side of the house. Such a barrier is most effective in reducirg the level of high-frequency sounds that travel in a beam-like manner. Its effectiveness for attenuating low-frequency sounds is limited by the ease with which such sounds diffract or bend around a barrier. At best, only a 5 to $10 \mathrm{~dB}$ reduction of the total sound level can be expected, but the attenuation of the high-frequency sounds may result in an appreciable reduction in annoyance. Since the acoustical efficiency of such a barrier may not be improved by massive construction, it nlay as well be made of relatively light material, such as 1 -inch $(2.5 \mathrm{~cm})$ thick boards.

\section{Reducing the Intrusion of Aircraft Noise}

Brief intrusions of high-level sounds are among the most troublesome to remedy. A recent study of aircraft flyover noise showed that reports of annoyance tended to 
increase by a factor of only two when the flyover rate increased by a factor of eight. Read of timistically, this means that if you can tolerate one intrusion, you probably can tolerate two. From the standpoint of an acoustical engineer who must quiet an airport area, and the community that must live within the area, the situation must be viewed pessimistically. Reducing the number of landings and departures per hour to one-half their original number may leave a large number of delayed and disgruntled passengers in remote airports that they did not wish to see, but still produce only an insignificant dent in the number of complaints from the residents. (For a householder, this statistic is also important. If you must operate a noisy tool, even though very briefly, it is important to try to stop the noise at the source. Otherwise, you may find yourself the recipient of a number of complaints despite the limited duration of the noise involved.) Moreover, some studies have shown that people do not become accustomed to noise intrusion as time goes on, but remain intolerant.

If you are one of many home owners who now find themselves constantly exposed to intense and highly disturbing aircraft noise, you should consider carefully the following four basic facts before you decide on any course of action to resolve the problem.

(1) The cost of soundproofing an existing home against intense aircraft noise is exceedingly high.

(2) Although soundproofing your home will reduce your annoyance indoors, it does nothing to improve the noise environment outside the home. Therefore if you enjoy gardening, patio parties or backyard barbecues, you will have to tolerate the aircraft noise as before.

(3) The probability is rather low of gaining noticeable relief from the noise in the near future through the development of quieter aircraft or changes in aircraft landing or takeoff flight procedures. In fact, the noise environment near a large airport generally tends to grow worse in time, with the continuing expansion in aircraft size and flight operations.
(4) The most effective solution may be to move to a more quiet area, even though it might entail some financial loss in the sale or rental of your home.

The following is a discussion of the various sound insulating modifications that may be used to reduce the intrusion of intense aircraft noise into the home. Figures 5-1 through 5-11 illustrate the structural details of such modifications.

Major Sound Insulating Modifications:

(a) Incorporate the noise-reduction recommendations given above relative to reducj.rig the intrusion of neighborhood and traffic noise.

(b) Windows

Windows are the weakest acoustical barrier in the exterior shell or walls of most homes.

Ordinary locked, double-hung windows generally provide an average noise reduction of about $18 \mathrm{~dB}$; the addition of a storm window, as illustrated in Fig. 5-1, will increase the sound insulation to about $24 \mathrm{~dB}$. Caulking of both sets of wirdows improves the sound insulation about $3 \mathrm{~dB}$ at the higher frequencies. However, if the aircraft noise still seems to be coming through the windows, despite the fact that they were equipped with storm windows and sealed air tight, then the existing window installation will have to be replaced with fixed, well-sealed, double pane windows throughout tha house.

As illustrated in Figs. 5-2 and $5-3$, the window installation then would consist of two 1/4-inch $(6 \mathrm{~mm})$ thick panes of glass, each encased in a U-shaped soft, resilient gasket with a 4-inch (10 $\mathrm{cm})$ air space between panes. Line perimeter surfaces within the framing with acoustic tile or other sound absorbent material.

This

double-window

installation when properly sealed would increase the sound insulation to approximately $37 \mathrm{~dB}$. 

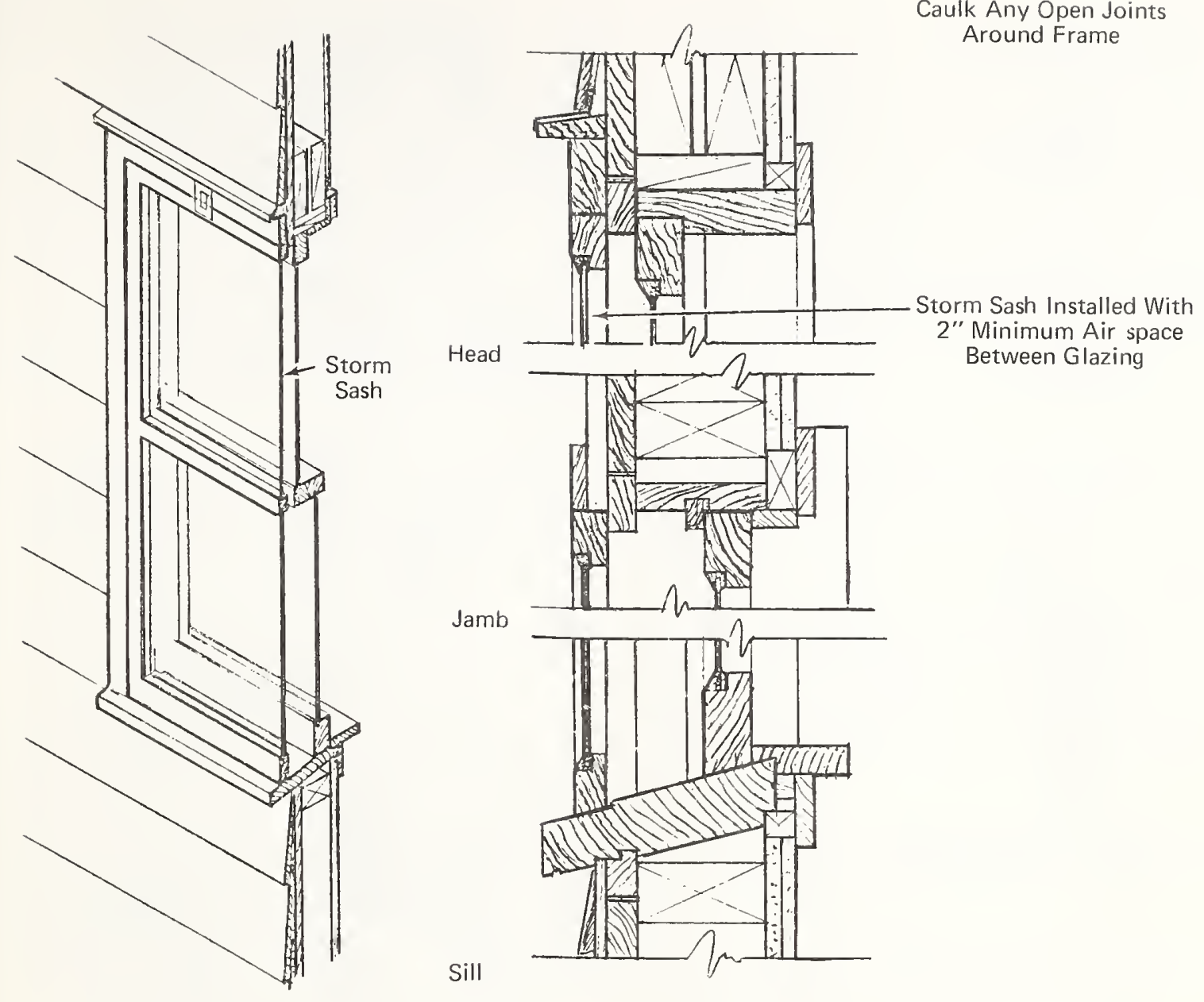

Fig. 5-1. DETAILS OF A DOUBLE-HUNG WINDOW WITH A STORM SASH 


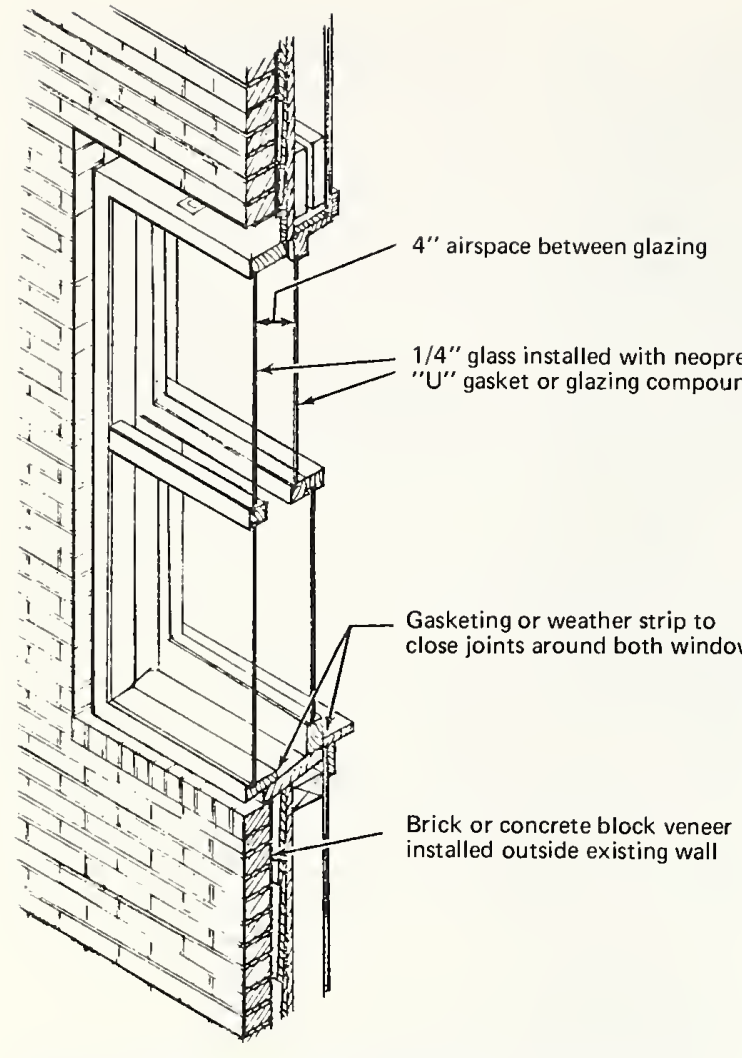

Fig. 5-2. INSTALLATION OF BRICK VENEER AND DOUBLE 1/4-INCH WINDOW GLAZING

\section{(c) Exterior Doors}

If the remaining noise now seems to be coming through the doors, remove the moulding from the door frame to see if there are any air gaps or noise leaks between the door frame and the wall. The conventional practice of installing prefabricated door kits, in which the doors are premounted in a door-frame assembly, often causes serious sound transmission problems. Builders generally provide an oversized opening to receive the preassembled door and fasten the assembly into place with a few wedges and nails, and completely ignore any resulting air gaps or leaks. These are conveniently covered and hidden from view by the thin finish moulding. However, such leaks must be sealed if any improvement in sound insulation is to be expected, and certainly before any existing doors are equipped with gaskets or replaced with soundproof doors.

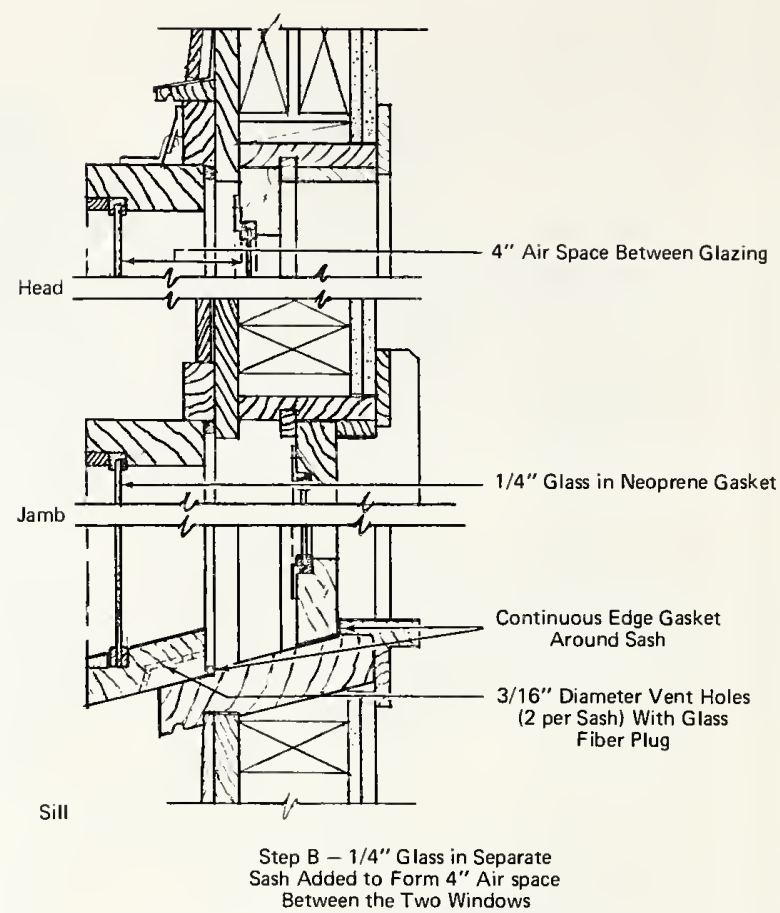

Fig. 5-3. DETAILS OF DOUBLE 1/4INCH WINDOW GLAZING

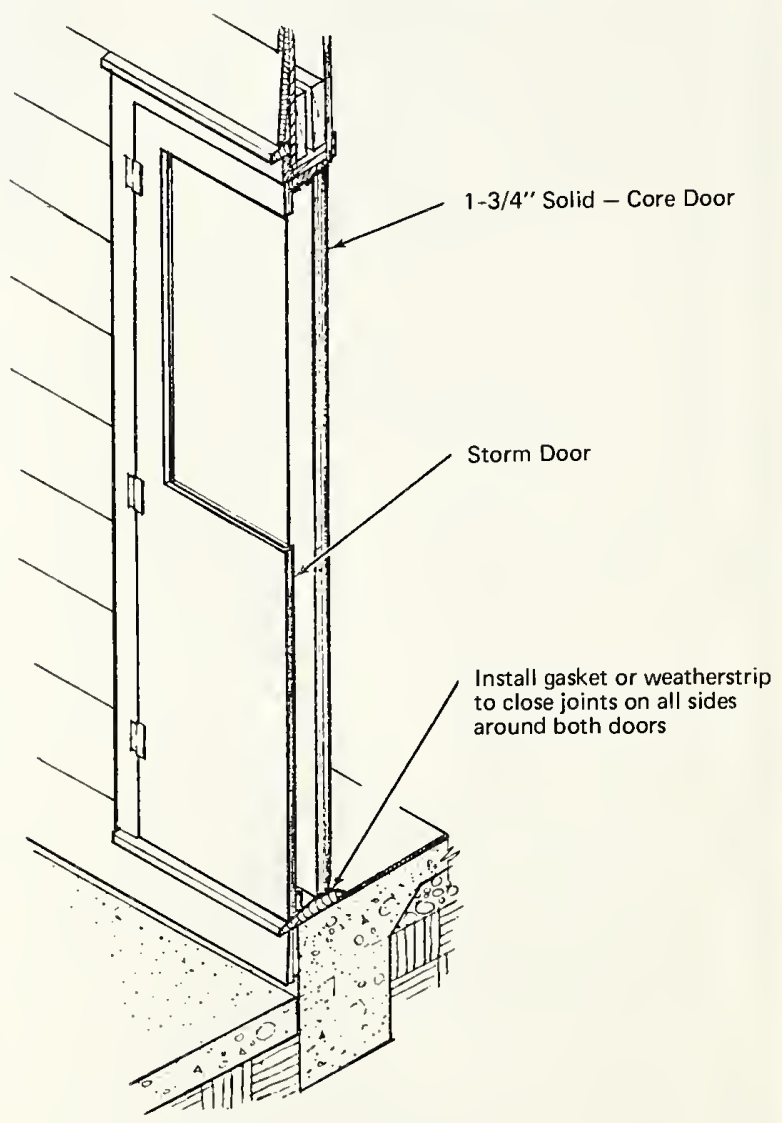

Fig. 5-4. DOOR MODIFICATIONS 
Replace existing doors with
$1-3 / 4$ inch $(4.5 \mathrm{~cm})$ thick solid-core doors with soft, resilient, perimeter gaskets and threshold seals. Install storm doors with 1/4-inch $(6 \mathrm{~mm})$ thick glass and weather stripping. It is estimated that the noise reduction provided by this combination of doors would be about $20 \mathrm{~dB}$ greater than that of a conventional exterior door. Details of these modifications are illustrated in Figs. 5-4 and 5-5.

(d) Exterior Walls

Generally speaking, exterior walls constructed of poured concrete, cinder block, brick or other masonry materials provide sufficient sound insulation against aircraft noise, if all penetrations through the walls have been properly treated and sealed. For example, ventilation ducts and exhaust vents must be lined with sound absorbent material or equipped with sound silencers; holes around penetrations of gas or water pipes, electrical conduits and refrigerant lines must be sealed airtight with a resilient, non-setting caulking compound. Settlement cracks in the walls or gaps around the framing of windows and doors should be caliked or sealed in the same manner.

No structural changes or modifications should be made to the exterior walls until you are sure that the noise is not entering the house through the basement, crawl space or attic areas.

Exterior walls of light-frame construction generally consist of wood studding faced on the inside with gypsum board and on the outside with insulation board and wood, metal or stucco siding. Such walls are inherently incapable of providing adequate sound insulation against moderately high levels of noise, much less against the intense levels of aircraft noise intrusion. Such walls would have to be capped on the outside with a

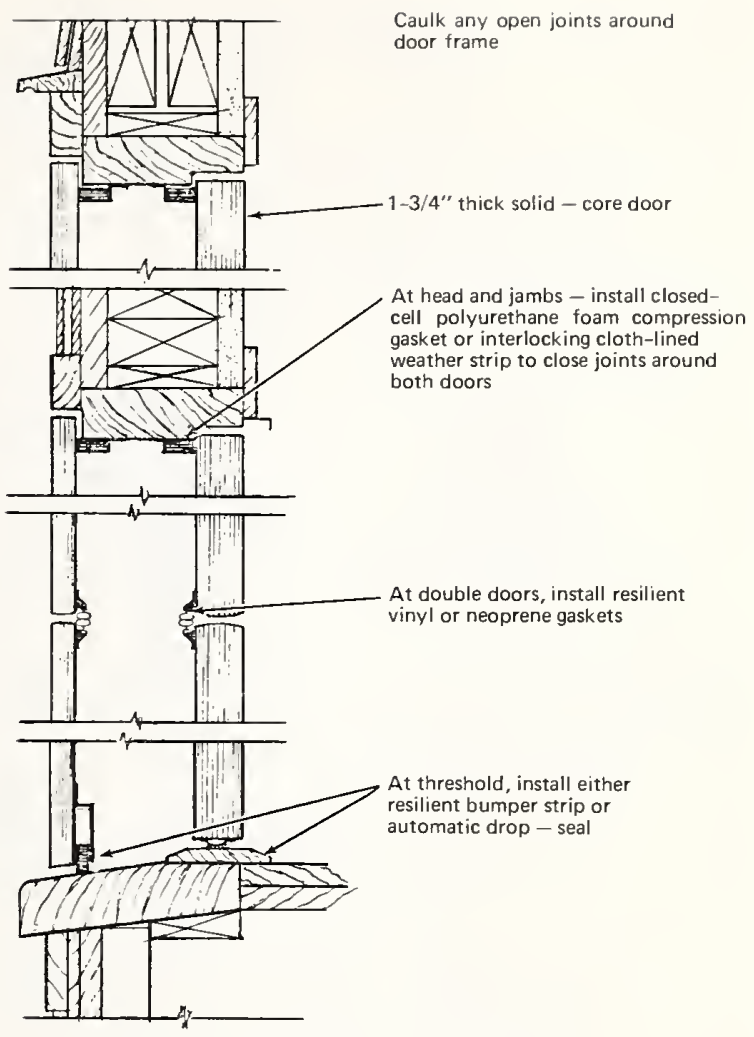

Fig. 5-5. DETAILS OF DOOR MODIFICATIONS

4-inch $(10 \mathrm{~cm})$ thick wall of brick veneer before the aircraft noise could be reduced to a tolerable level inside the house, as illustrated in Fig. 5-2. Figure 5-6 illustrates the details and sound insulating effertiveness of other window-wall constructions.

(e) Roof and Ceiling Modifications (see Figs. 5-7, 5-8, 5-9 and 5-10)

\section{Ventilated attic space}

If the attic is to be used as a walk-in storage area, install subflooring on top of the ceiling joists and cover with 5/8-inch (16 $\mathrm{mm}$ ) thick gypsum board. Tape and seal the edges and joints of the gypsum board layer. Otherwise, the gypsum board may be installed directly on top of the ceiling joists.

Install a metal duct vent attenuator, as illustrated in Fig. 5-11, at all air-circulation vents in the attic. All other openings 
Movable Windows

Casement or Double Hung

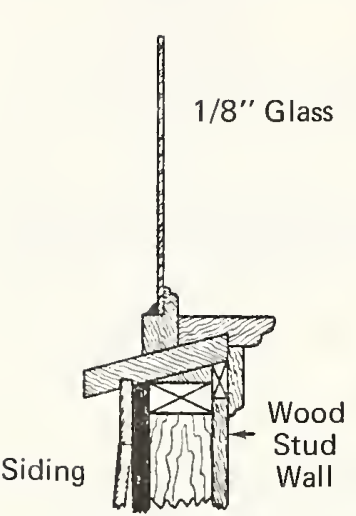

Fixed Windows

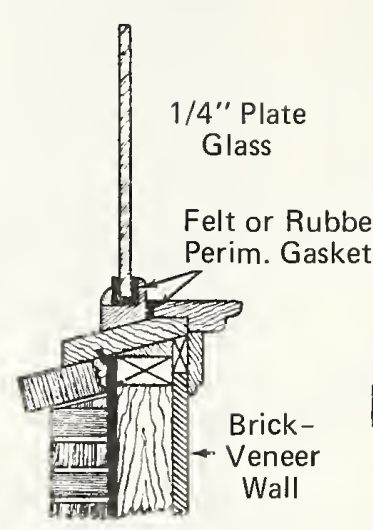

\section{rixed Windows}

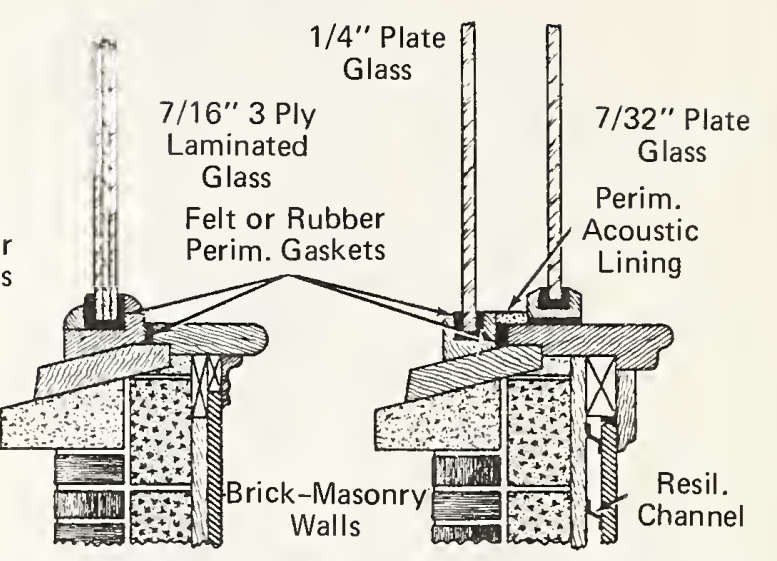

\begin{tabular}{|c|c|c|c|}
\hline Window: Poor & Fair & Good & Very Good \\
\hline Wall: Poor & Fair & Good & Very Good \\
\hline
\end{tabular}

Fig. 5-6. SOUND INSULATION EFFECTIVENESS OF VARIOUS WINDOW-WALL ASSEMBLIES

or cracks should be sealed with glass fiber wadding and resilient caulk.

Plank and beam vaulted ceiling or flat roof

Attach wood furring or preferably metal resilient channels to the existing ceiling. With self-tapping screws, fasten 5/8-inch $(16 \mathrm{~mm})$ thi.ck gypsum board to the furring or metal channels. Tape and finish joints and edges of the gypsum board in the conventional manner.

\section{(f) Pasement Areas}

Caulk or weatherstrip existing windows and install storm windows.

Weatherstrip existing hinged or sliding doors and install storm doors.

(g) Craw1 Spaces (see Fig. 5-11)

Insta11 a metal duct vent attenuator with 1 -inch $(2.5 \mathrm{~cm})$ thick glass fiber liner at a 11 air-circulation vents. Seal al1 other openings or cracks with glass fiber packing and resilient caulk.

Instal1 5/8-inch (16 $\mathrm{mm})$ thick exterior plywood or moisture-proofed gypsum board to the underside of the floor joists.

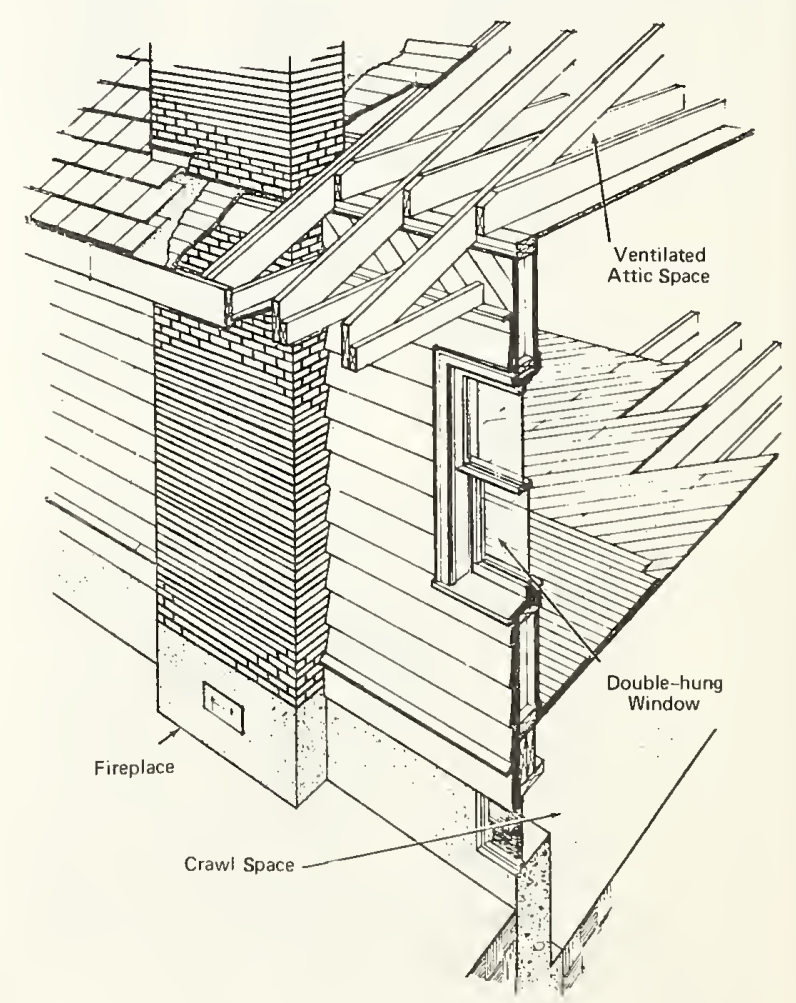

Fig. 5.7. HOUSE WITH A CRAWL SPACE AND VENTILATED ATTIC 


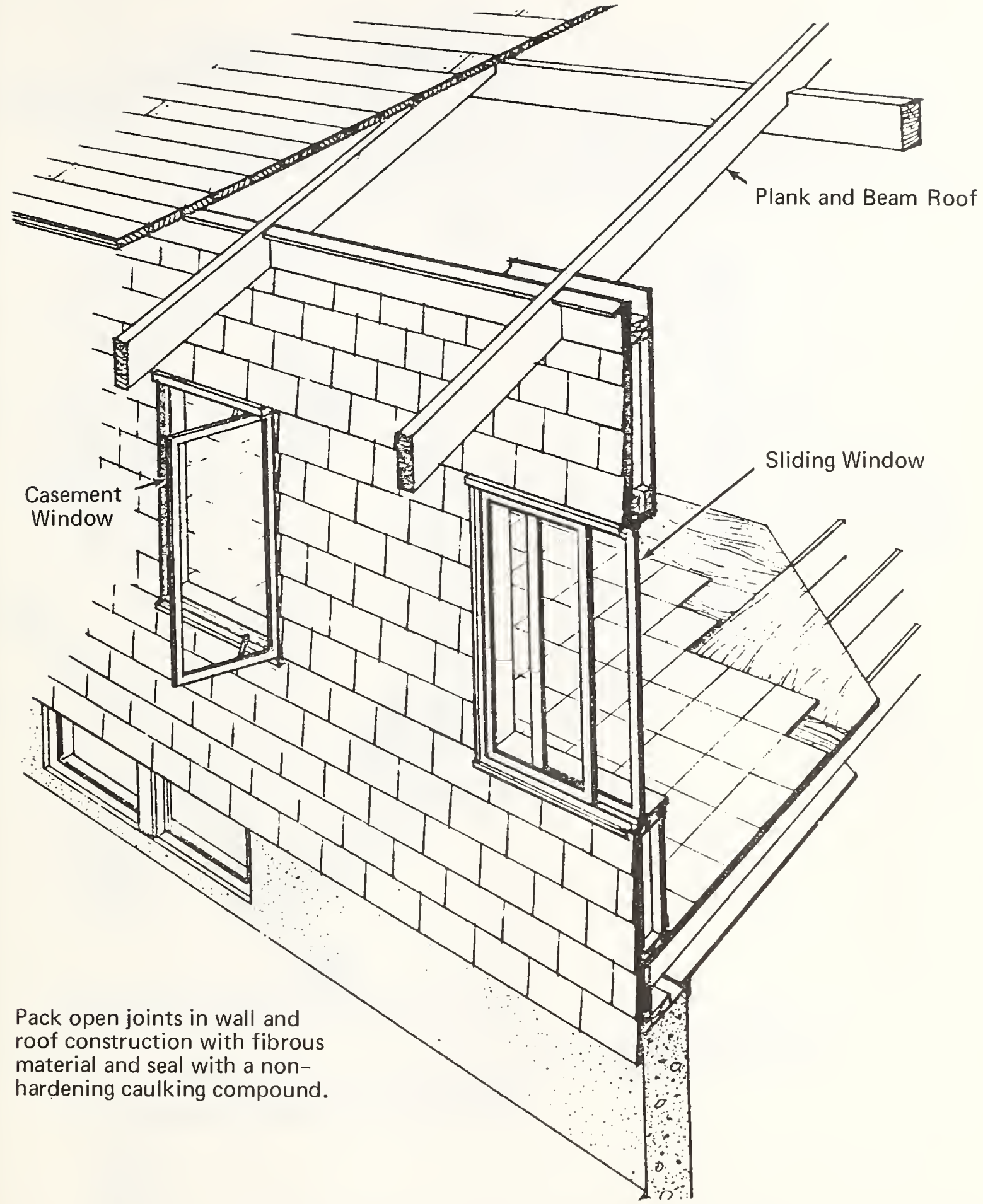

Fig. 5-8. HOUSE WITH A BASEMENT AND PLANK AND BEAM ROOF 


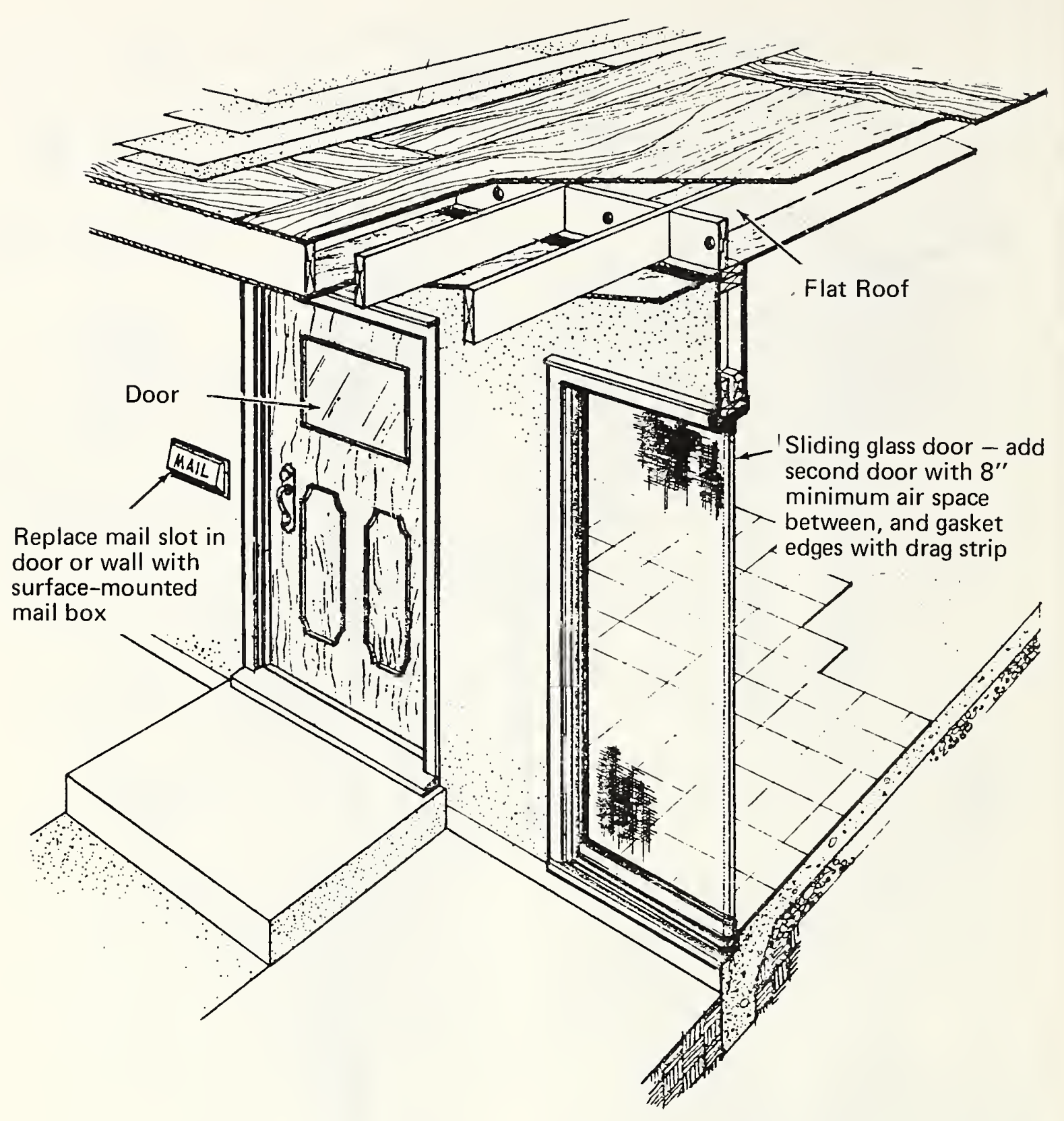

Fig. 5-9. HOUSE WITH SLAB ON GRADE AND A FLAT ROOF 


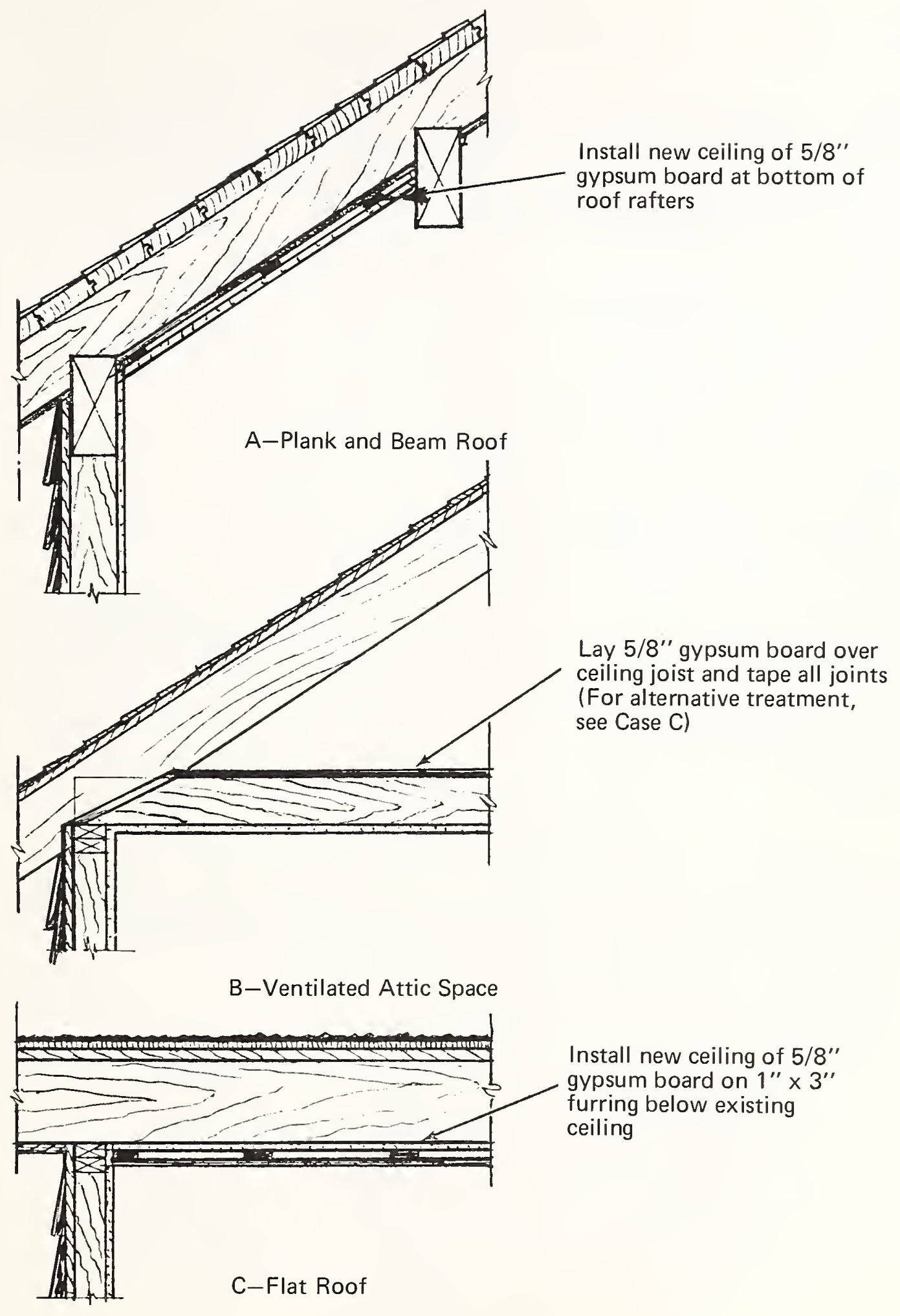

Fig. 5-10. ROOF AND CEILING MODIFICATIONS 


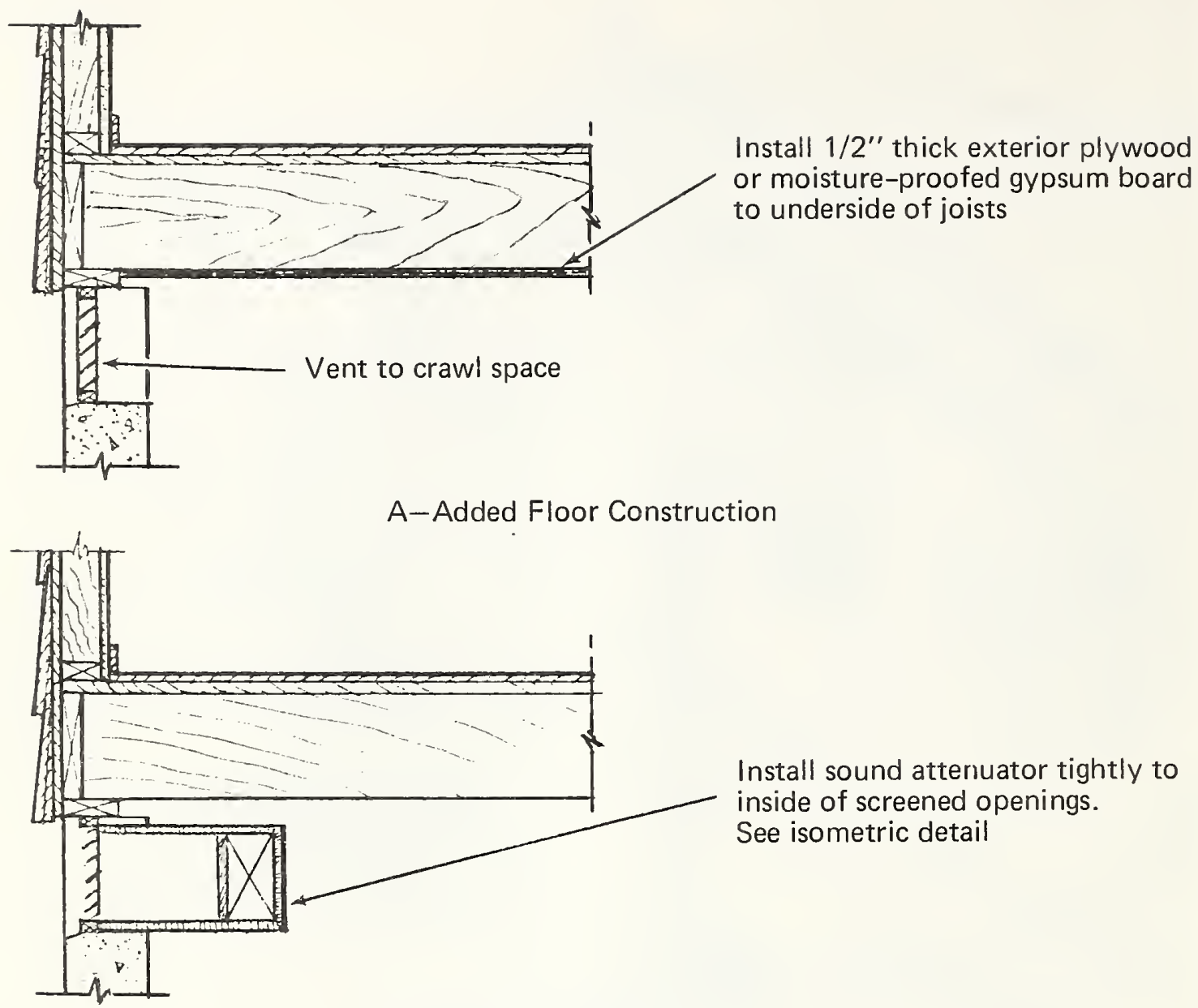

B-Vent Attenuators

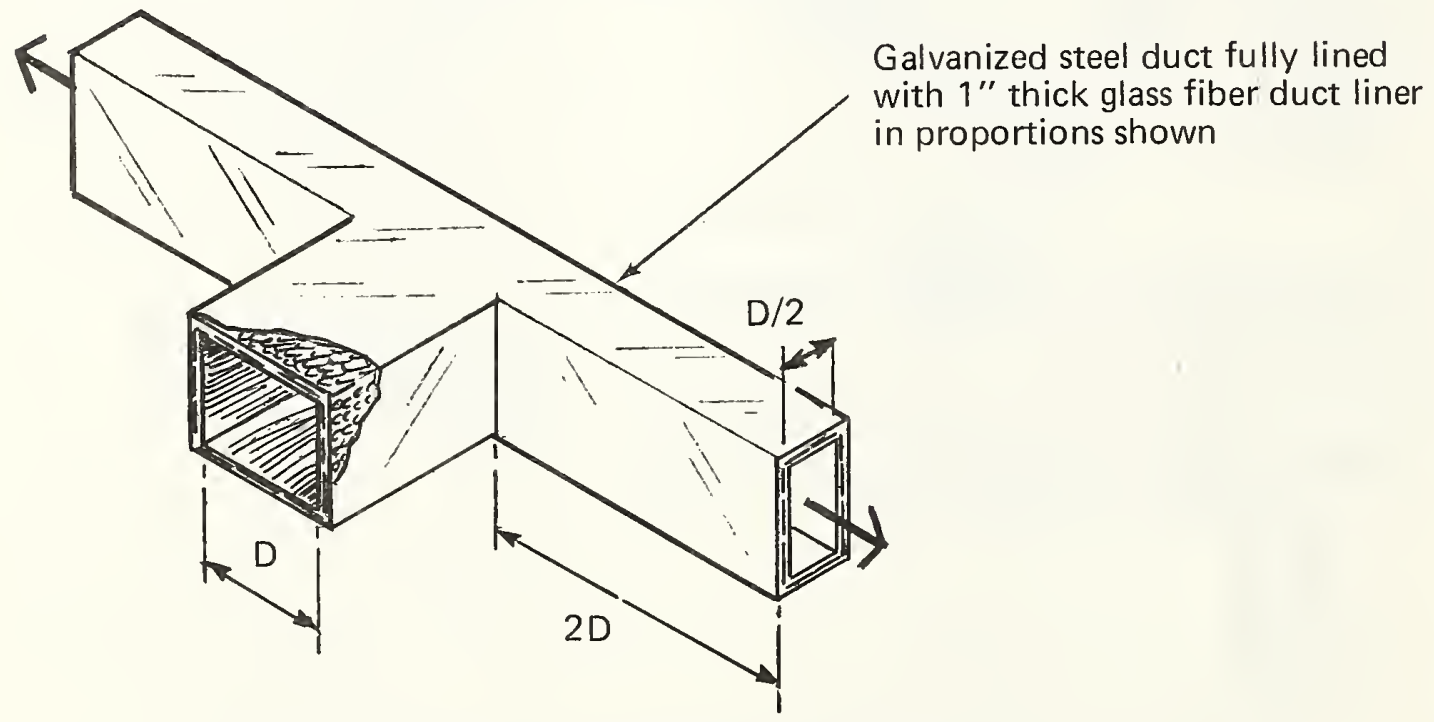

Fig. 5-11. CRAWL SPACE MODIFICATIONS 
SELECTING A QUIET HOME OR APARTMENT

(or how to avoid making the same mistake twice)

If you are planning to move to a new home or apartment and desire more peace and privacy than you have at present, the following recommendations can help you to select a quiet location. However, you should be careful to consider all factors before making your move, because the desirability of living in a quiet community may be offset by some disadvantages. For example, it might take you twice as long to drive to work or mid-city attractions from the new location than it does from your present one.

\section{SELECTING A QUIET RESIDENTIAL AREA}

(a) Obtain Maps of the Area

Obtain a recent edition of a detailed map of the area that you propose to live in and study it carefully for the location of major sources of noise. Look, particularly, for such major noise producers as airports, railroads, expressways, and industrial areas.

Airports: If possible get a sectional aeronautical chart of the area to determine the location of all airports and their respective flight patterns and air routes relative to the proposed building site. Such charts may be obtained from local airport authorities or the Aircraft Owners and Pilot Association, Washington, D. C. 20014 .

Sites near large commercial, international or military airports should be avoided if possible. Residential buildings directly in line with prescribed runways, take-off and landing patterns or flight paths would have to be located at least 15, and preferably 20 miles (24 to $32 \mathrm{~km}$ ) away from the airport before the noise from jet aircraft fly-overs would be relatively unnoticeable to the occupants. (See Fig. 6-1.)

Near an airport, but off to the side of the runways, buildings would need to be a lateral distance of about 3 to 4 miles ( 5 to $6 \mathrm{~km}$ ) from the flight path before the noise would drop to a tolerable level at the building location.

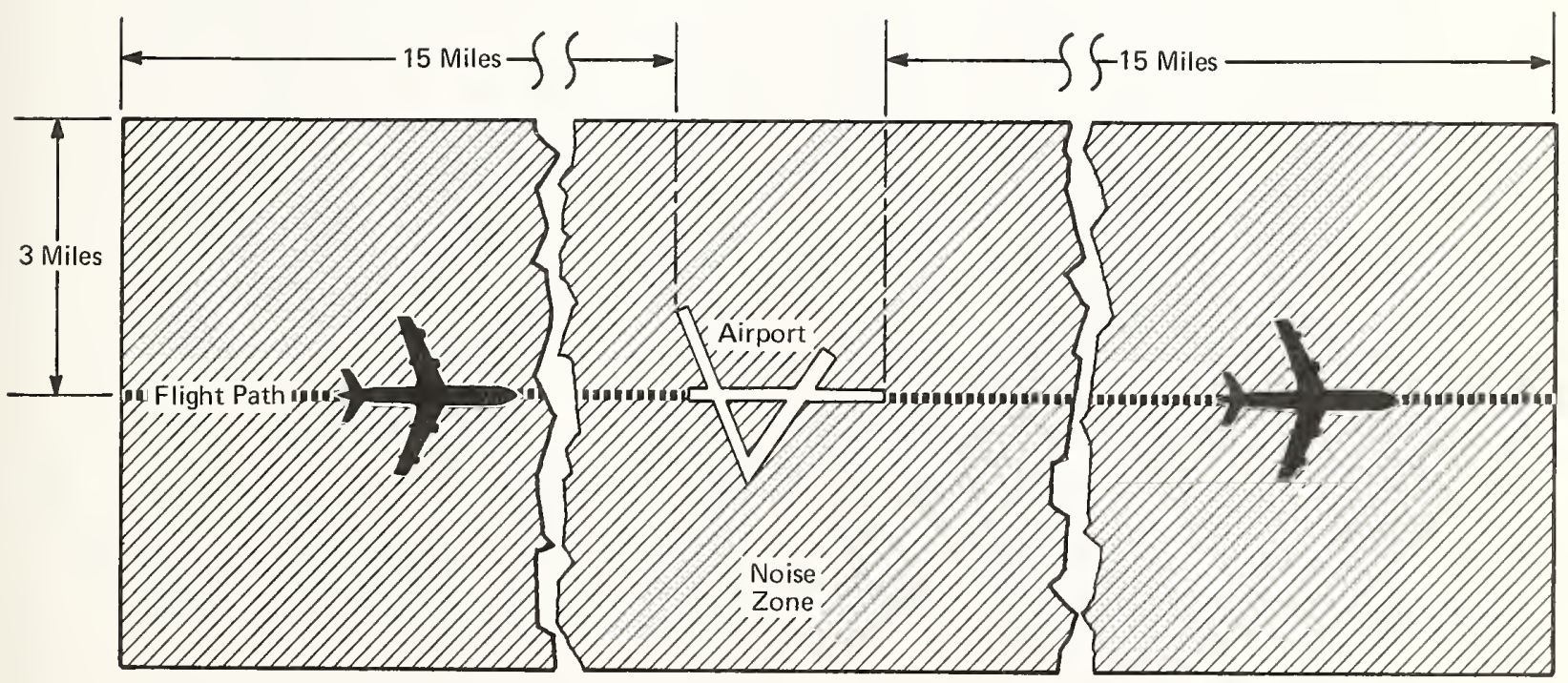

Fig. 6-1. TO AVOID AIRCRAFT NOISE DISTURBANCE, SELECT HOMES OR APARTMENTS OUTSIDE SHADED AREA 
Railroads, Expressways and Industrial Areas: If annoyance or disturbance from these excessively noisy sources is to to be avoided, residential buildings should be located at a distance of at least one mile and preferably two miles $(1.6$ to $3.2 \mathrm{~km})$ from the sources.

(b) Check With City Zoning or Planning Authorities

Consult with local city or county planning authorities regarding future development plans for the area. As a result of future rezoning action, quiet residential areas frequently are bordered by industrial parks or huge shopping malls. Although such business centers by themselves may not cause any annoyance, the noise from the increasing flow of automobile traffic and shipments by heavy trucks to and from the areas, especially at night, may become a major source of disturbance for you.

(c) Consult With City or County Transportation Authorities

Such authorities should be consulted relative to the status and location of existing and proposed expressways, mass transit systems and changes in aircraft flight paths, procedures and operation.

\section{(d) Conduct An On-Site Examination}

Conduct several on-site examinations of the area during daylight and especially after midnight to assess the outdoor noise environment. Be especially alert for the location of fire and police stations, schools and athletic fields, hospitals, sport arenas, race tracks, shopping centers and amusement parks. Although ordinarily these civic centers and activities may be considered secondary sources of noise, they could well become, depending on their proximity to your location, the major source of noise and disturbance for you. (e) Consult With Residents Living In The Area

It often pays to talk to people living in the area to determine whether or not they are disturbed by noise, especially at night.

\section{SELECTING A QUIET LOCATION WITHIN A HOUSING OR APARTMENT DEVELOPMENT}

of course the first thing that you should do is to select and inspect an apartment development that advertises "soundproof" wall and floor construction, quiet, efficient central airconditioning systems and fully carpeted interiors, including entrance foyers, corridors and hallways.

In addition, it is important to choose an apartment that has a ventilation system with air supply and return branch ducts serving individual rooms, and well-fitted, solid-core doors with perimeter gaskets and threshold seals installed in bedroom areas. Such construction and furnishings are prerequisites for ensuring a quiet environment and adequate privacy within a dwelling unit, as well as minimizing the intrusion of noise from neighboring apartment units or other areas in the building.

If possible, avoid choosing a home or an apartment unit that has only a single air-return grille or duct to serve the entire dwelling area. In order to complete the circulation, entrance doors to individual rooms must be undercut about 1 inch $(2.5 \mathrm{~cm})$ at the bottom to provide an air gap for passage of the return air. Regrettably, such air gaps also create a series of flanking paths throughout the dwelling, which virtually nullify any sound insulation that partition walls and doors separating rooms could provide. If, in addition, the return grille is undersized, the air-flow velocity at the face of the grille must be made fairly high in order to maintain adequate ventilation. This, unfortunately, may generate a considerable amount of high-pitched noise. As a consequence, the occupants of such dwellings frequently complain not only about the lack of privacy, but also of interference to sleep caused by the grille noise intruding in to the bedroom area.

A particularly serious problem that often occurs in apartment buildings is caused by a ventilation system in which a 
common air return is installed in the corridor outside the dwelling units. The entrance doorways of all apartment units must then be undercut to permit the venting of the return air. Obviously, the privacy between adjacent apartments is seriously breached in such cases.

The only remedy the tenant himself has is to close the gap at the bottom of each door in his dwelling with a threshold plate equipped with a tubular gasket. He must then install in the wall or ceiling of each room an acoustically lined air-transfer duct to vent the air into the adjacent hall or corridor. Both door thresholds and air-transfer ducts are commercially available. Techniques for sealing door thresholds and installing air-transfer ducts are discussed in Chapter 4 and illustrated in Figures $4-7$ and $4-12$, respectively.

Although the intrusion of outdoor noise is prevalent in dwellings in urban locations, it can occur in dwellings in

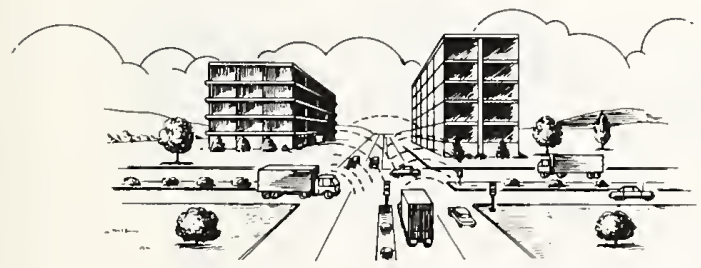

Avoid building sites at intersections of major traffic arteries. Such sites are extremely noisy due to accelerating, decelerating, and braking vehicles.

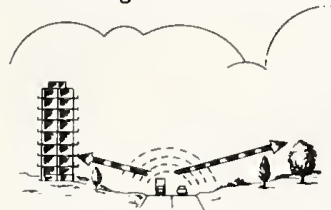

Building sites in open areas are less noisy than sites in congested building areas.

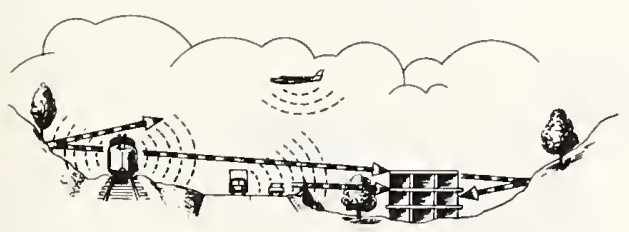

Avoid hollows or depressions. They are generally noisier than flat open land. supposedly quiet suburban and even rural areas. While an occasional jet aircraft flyover or a passing truck may cause some disturbance in such areas, many problems arise from a neighbor's air conditioning unit, barking dogs or bird calls. If the intrusion of such noise is bothersome to you, give preference to buildings that have fixed or well-sealed windows that are thermally insulated or feature double-pane construction. Buildings with single pane windows protected by well-fitted storm windows also would provide an adequate degree of insulation against such noise. The intrusion of outdoor noise may be minimized even further by observing the following suggestions relative to selecting the building site.

(a) Avoid building sites that front on main traffic arteries or bus routes. Early morning traffic noise may be a source of annoyance, as illustrated in Fig. 6-2.

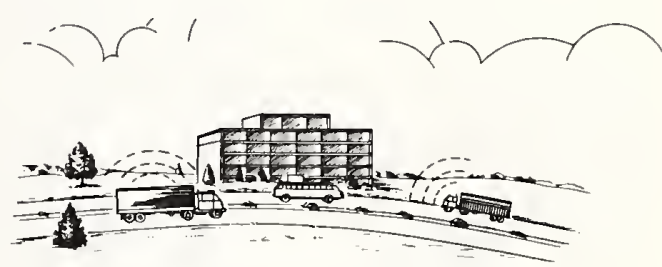

Avoid building sites on the crests of hilly traffic arteries. Such sites are very noisy due to low gear acceleration noise.

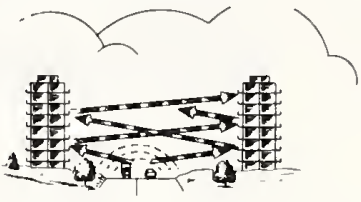

Traffic arteries between tall buildings are quite noisy. Avoid building sites opposite tall buildings in such cases.

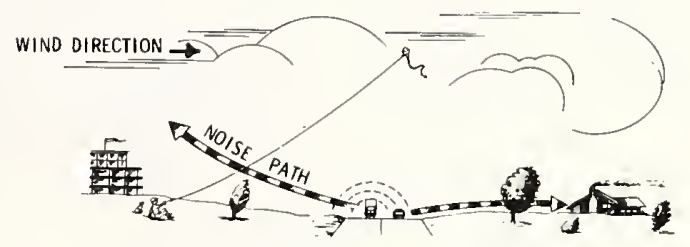

Upwind building site is less noisy than a downwind site.

Fig. 6-2. EXAMPLES OF APARTMENT SITES THAT SHOULD BE AVOIDED 

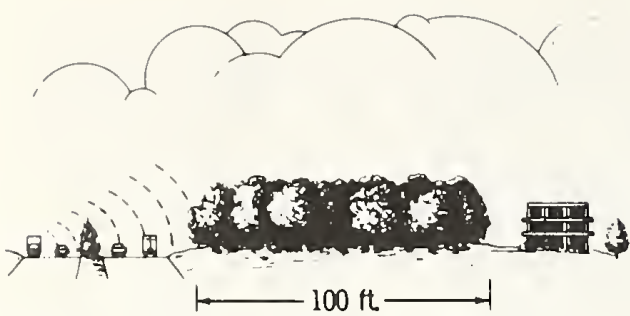

A thick growth of leafy trees \& underbrush reduces noise about 6 to $7 \mathrm{db} . / 100 \mathrm{ft}$. (average over audible freq. range)

Low Freq. Loss: 3-4 db

High Freq. Loss: 10-12 db

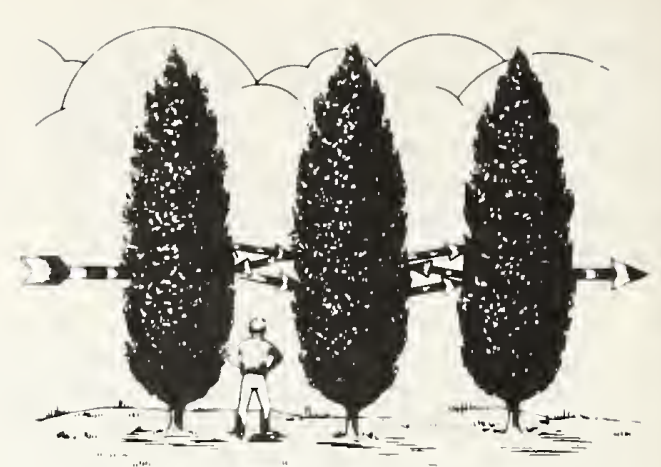

High Freq. Reduction 3-4 db

Single row of trees is worthless as noise barrier. Due to inter-reflection multi-rows of trees are more effective.
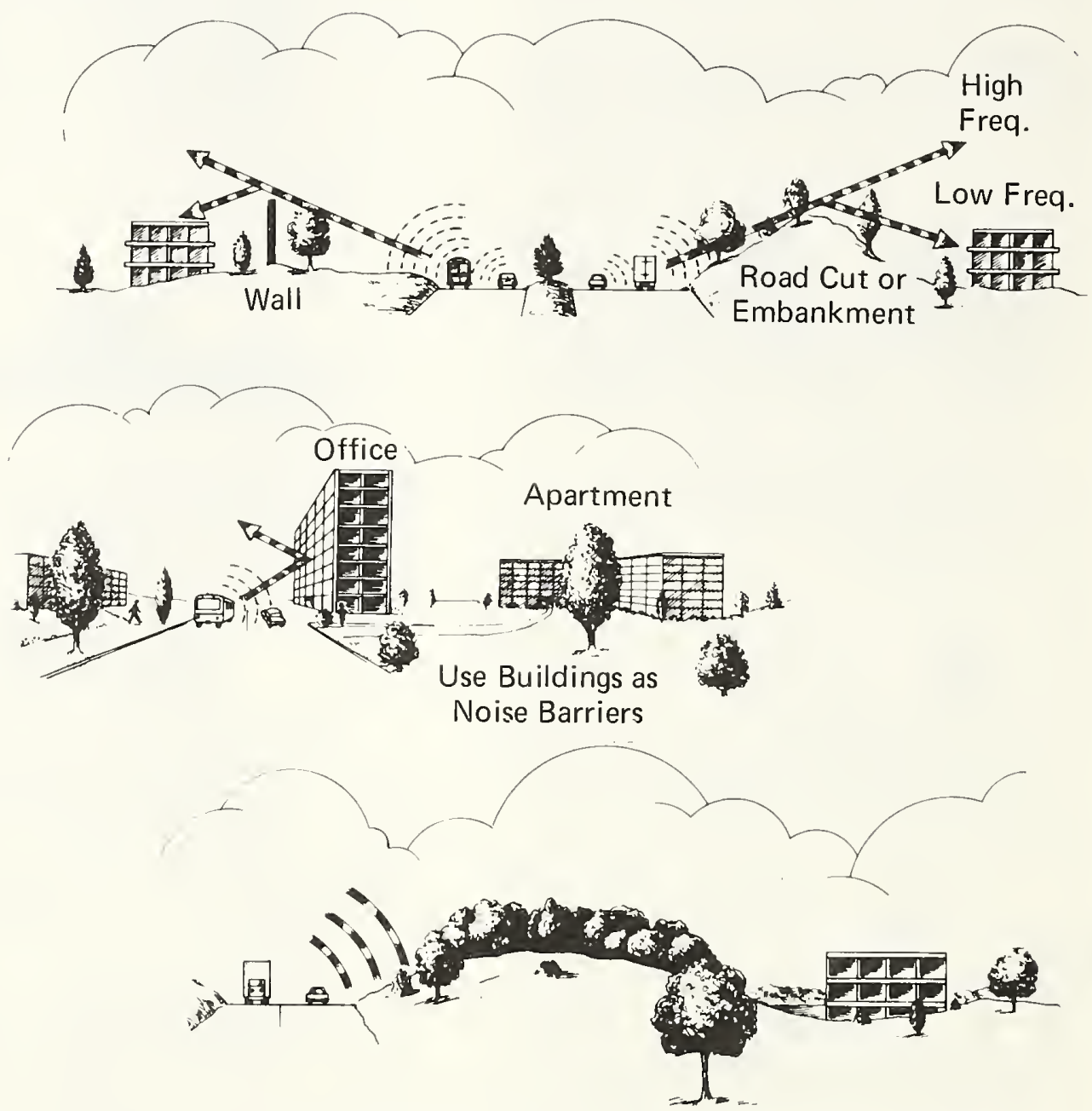

Fig. 6-3. ACOUSTICAL SHIELDING PROVIDED BY LANDSCAPE AND NATURAL AND MAN-MADE BARRIERS 
(b) Select building sites that face wide open spaces such as parks, golf courses, rivers, lakes and seashores. Such areas usually are very quiet, especially at night.

(c) Choose a building site that is located as far as possible or is acoustically shielded from a major source of noise by other buildings, natural landscaping or wooded areas, as illustrated in Eigs. 6-3 and 6-4.

3. SELECTING A QUIET APARTMENT UNIT WITHIN THE BUILDING

Unless the building manager can demonstrate that the apartment units will provide an adequate degree of insulation against the intrusion of noise from other areas within the building and noise of outdoor origin, the renter should take the following precautionary measures to ensure freedom from such disturbances.

(a) Select apartment units that are remote from elevators, mechanical equipment rooms, laundry rooms, indoor garages, or roof-mounted ventilation equipment, such as chiller pumps, compressors or cooling towers. Rarely are such powerful noise sources properly enclosed in sound-insulating structures or vibration-isolated from the building structure. As a consequence, dwelling units adjoining such sources may be subjected to intermittent round-the-clock intrusion of excessively high noise levels. These dwellings can readily develop into an acoustically intolerable environment.

(b) Apartments that overlook parking lots, thoroughfares or through streets, playgrounds and swimming pools may be noisy during parts of the day unless carefully sealed against noise. Noises caused by automobile operation such as starting of engines and door slamming, particularly during the

early morning hours, can be a source of annoyance. Likewise, the voices or shouting of children at play throughout the day and evening may be disturbing. If possible, give preference to an apartment unit on the quiet side of the building; that is, one which faces away from such noise sources.

(c) Consider the surroundings of apartments that face U-shaped courtyards. Such areas teno to be reverberant and can be noisy, particularly if they are used as recreation or play areas or face main highways or traffic arteries.

NOT THIS
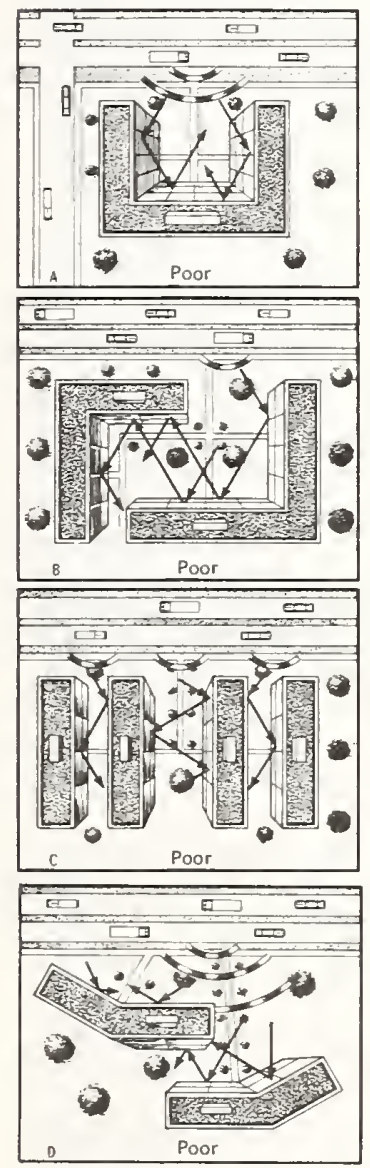

Fig. 6-4. ORIENTATION OF APARTMENT BUILDINGS FOR

OPTIMUM ACOUSTICAL SHIELDING

THIS
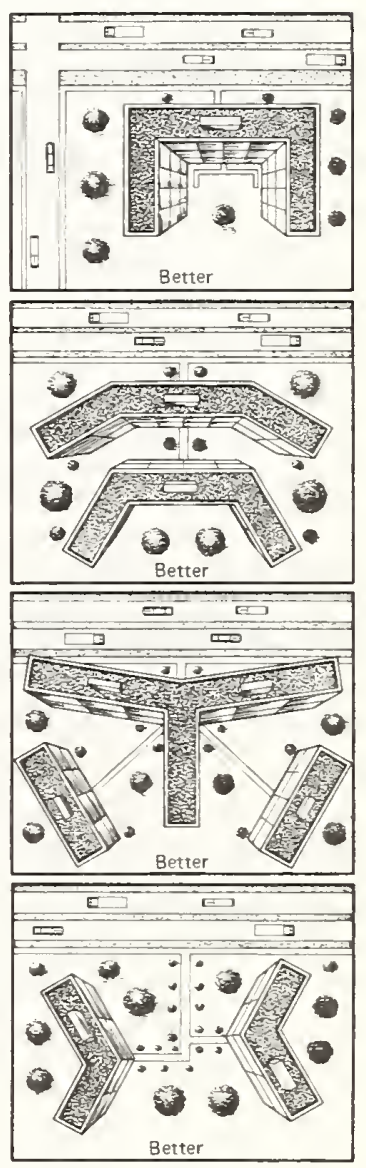


\section{ANALYZING THE PROBLEM:}

If you are faced with a noise problem, examine it first in terms of the three basic elements: source, path, and receiver. We have discussed these elements in Chapter 3. obviously, the ideal solution is to reduce the noise output of the source, itself, since this will benefit both the operator of the noisy equipment and bystanders or other people in the vicinity.

If attempts to reduce the noise output of the source have proved only marginally successful, it may be feasible to alter the transmission path so that less noise reaches the listener. Acoustical absorbers and noise barriers may be used to attenuate the noise before it can reach noise-sensitive areas. Unfortunately, such measures do not provide much relief for the operator of the noisy equipment, who usual1y is immersed in the direct noise field from the machine. His only remedy is to wear ear protectors, and to minimize the duxation of each exposure to noise.

Suppose you find yourself the unwilling receiver. In the first place, do not risk your hearing unnecessarily while waiting hopefully for something to be done. The most important action is to determine whether the noise can be hazardous to your hearing, or whether it is merely bothersome. This you can do simply by using your ears, and making a few observations. Follow this simple rule of thumb; If THE NOISE LEVEL IN YOUR SURROUNDINGS IS SO HIGH THAT YOU HAVE TO SHOUT TO CONVERSE WITH A PERSON NEARBY, CONTINUED DAILY EXPOSURE AT THIS LEVEL FOR MANY HOURS MIGHT ENDANGER YOUR HEARING. However, to be on the safe side, a closer estimate of the noise level in the area is required. You can estimate the noise level from the limiting distances over which intelligible conversation is possible, and the vocal effort required to maintain it.

Figure 7-1 shows the empirical relationship that has been found between the distance over which spoken words can be recognized and the typical sound level of the environment, as one would read it on a sound level meter using the A-weighting scale. As an example of the use of the graph in Figure 7-1: Suppose you are talking to a person at a customary distance of about 5 feet $(1.5 \mathrm{~m})$ from him. If you can carry on your conversation - with no unusual effort to hear him or no feeling that you need to raise your voice to a higher level - then it's likely that the sound level does not exceed $67 \mathrm{~dB}(\mathrm{~A})$ where you are standing [point (1), Fig. 7-1]. Suppose now you are at a lively cocktail party which gradually becomes so noisy that you have to raise your voice to converse and have trouble understanding your neighbor [this condition is at point (2)] - the noise level at this location would be approximately $75 \mathrm{~dB}(\mathrm{~A})$.

If by chance you were to find yourself in an industrial plant so noisy that you must shout to be understood at a distance of 5 feet $(1.5 \mathrm{~m})$, [point (3)] -- the noise level would be about $90 \mathrm{~dB}(\mathrm{~A})$. The current national standard on occupational noise restricts an employee's exposure to $90 \mathrm{~dB}(\mathrm{~A})$ as an eight hour time-weighted average. From your own experience you can judge the advantages of maintaining environmental noise below this level.

To use Fig. 7-1 as a guide for estimating the level of noise in any indoor or outdoor environment, start a conversation at a normal voice level with a companion, and then back away. Arrange to signal to one another the moment you feel that extra effort is involved, either in speaking or listening. At this point, if both of you have normal binaural hearing, the distance between you can be noted on the horizontal scale of the graph. From the solid lire on the graph you can read off the effective A-weighted sound level in decibels along the vertical scale. This chart applies to tests conducted in a fairly reverberant space; only a very long echo time, as found in a large convention hall or church, will lead you to overestimate the sound level by compellirg you to override the echoes of your own voice.

To trouble-shoot noise problems in the home, first listen carefully to the noise for distinctive clues or characteristics that might identify the cause or suurce of noise. If the source is well known to you, you can recognize how the transmission has altered its sound where you are, and thus can tell whether it is reaching you by air or through the structure. Airborne sounds usually arrive with their high-pitched 


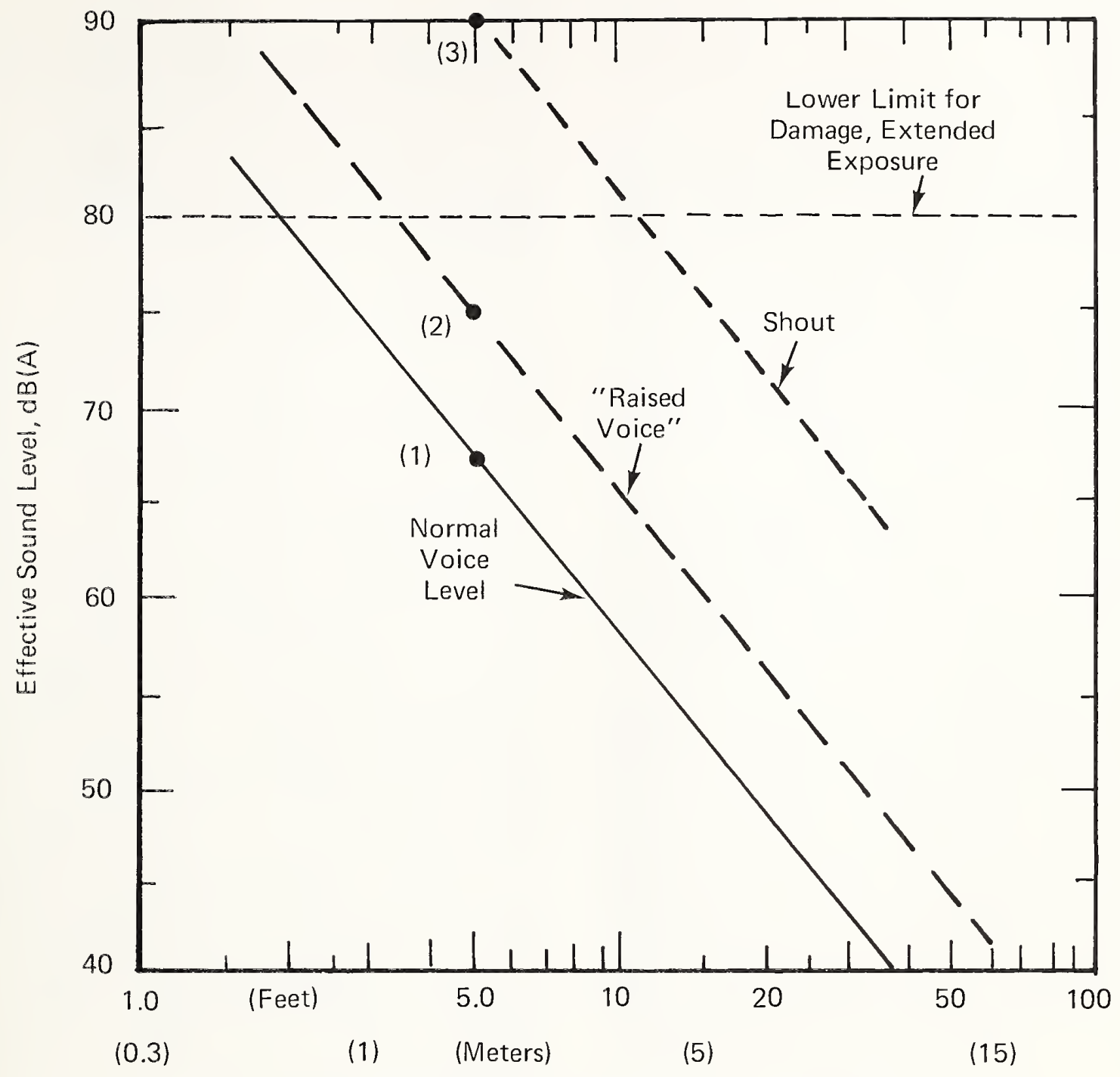

Limiting Distance for Conversation, "With Ease"

Fig. 7-1. THE "A" WEIGHTED SOUND LEVELS OF SPEECH AT VARIOUS DISTANCES UNDER ORDINARY CIRCUMSTANCES

components intact; structure-borne sounds are likely to seem muffled.

Some noise problems are relatively easy to recognize, especially if both the source and the noise transmission path are obvious -- as in the case of a dishwasher which operates in the kitchen but transmits much noise through the air nearby and sends vibrations along the plumbing system to other areas of the house. However, most noise problems are much more subtle and exasperating to cope with because of the difficulty in determining -- let alone locating -- the noise source or its dominant transmission paths. Such problems are particularly difficult in large high-rise apartment buildings, in which the noises generated by the numerous types of equipment, appliances, and occupant activities occur simultaneously. They combine and blend, and thus camouflage the offending sources.

If noise intrudes into your living quarters from an adjacent apartment, or perhaps from one room to another within your own dwelling, the following steps should 
help you to analyze and to alleviate the problem.

HOW TO LOOK FOR NOISE LEAKS:

In a high percentage of cases, the main cause of noise transmission problems within homes or apartments, or between adjacent dwelling units, is an airborne sound transmission path or leak. Look for holes, cracks or openings around pipe and duct penetrations, plumbing fixtures, ventilation grilles, back-to-back cabinets, and doors. Carefully examine the ceiling edges and corners of walls for cracks caused by building settlement. Even hairline cracks will permit passage of a considerable amount of noise.

For problems involving the intrusion of outside noise, check the effectiveness of gaskets or weather-stripping around the windows and exterior doors. However, the places that most frequently are overlooked, but are sources of serious noise leakage, are concealed behind the thin molding that frames doorways and windows. In conventional building practices the openings cut for the installation of pre-assembled door and window units are much larger than need be. These openings, which rarely are adequately sealed for acoustical or thermal purposes, present a direct noise transmission path. Sometimes, air spaces between door jambs and the wood door-stops which hold the gaskets, by-pass the gaskets and thus cause the noise leaks. In other cases, large holes in exterior cinder block walls may be hidden from view by thin veneer paneling. Although a visual examination of a room should be conducted to detect the more obvious noise leaks, it can not be fully relied upon to locate those hidden from view.

\section{USE YOUR EARS:}

When used properly, your ears are highly versatile instruments. Not only can they identify noise sources, but they can locate noise leaks and trace transmission paths, as well.

If you have trouble detecting a noise leak, cupping your hands and placing them behind your ears will increase the sensitivity of your ears and enhance your sense of sound localization. If, with your hands cupped behind your ears, you turn your head from side to side, you should find yourself able to locate more precisely the direction from which the sound or noise is coming. High-frequency sounds, which tend to be propagated in a beam, can be located rather easily this way. If the sound is very high-pitched, sealing one ear with your hand or finger tip and listening with the other ear as you turn your head may enable you to "home $i n$ " on the sound much more rapidly. Any path along which the noise grows louder will usually lead you to the area where the noise enters the room.

Having located the point of entry, you need next to determine how the noise enters the room. Is it transmitted through an air leak such as a crack, hole or an opening in a wall, or by structural vibration of building components such as a wall, or by both? One way of making this determination is to conduct a "talk test" with a companion located on the cther side of the wall. If the sound of his voice comes through the wall loud and clear, it is quite evident that there is a serious air leak in the wall. On the other hand, if you can hear him talking but the sound is garbled and you cannot understand what he is saying, the sound most probably is being transmitted structurally through the wall. The high-frequency sounds of speech, such as those characterizing the consonants which make speech intelligible, do not pass readily through wall or floor structures.

Another test permits you to estimate whether noise is being transmitted through a wall by way of air leaks or by way of structural vibration. Plug one of your ears with your fingertip and press the opposite ear tightly against the wall. Compare the loudness of the sound you hear while your ear is sealed against the wall with what you hear as you withdraw your ear from the wall. If the sound you hear with your ear pressed against the wall is louder, the sound is most likely travelling a structural path through the wall. However, if the sound is no softer when you withdraw your ear, the sound is probably reaching you through an air leak.

\section{USE HOME APPLIANCES AS DIAGNOSTIC TOOLS:}

Generally speaking, the task of resolving a noise problem often requires devices for generating sounds and vibrations of relatively high intensities. Fortunately, the average household contains a number of rather common appliances that can be used for such purposes. For example, vacuum cleaners equipped with rug beaters or power drills with drill bits placed slightly off-center are excellent sources of airborne 
sound and vibration. Transistor radios, electric shavers and food blenders are convenient sources of mid-frequency sounds.

Toy whistles and alarm clocks may be used for the generation of high-frequency sounds. Golf balls and walking canes can be used to generate fairly repeatable impact type noise when dropped from a constant height. The noise and vibration generated by all these sources cover a useful range of the frequency spectrum with an adequate intensity level for most investigations.

With the noise sources at hand, you now need a few simple noise-leak detectors and vibration sensors. Several devices that can perform these functions also can be found in most households. Appliances such as flashlights, hand mirrors, hairdryers, vacuum cleaners with crevice tool attachments, long thin knife blades, and even strips of thin stiff paper can be used quite effectively to locate simple noise leaks even without the noise source.

If you suspect noise leakage through a partition wall, try the following test. With a long thin knife blade or a strip of thin flat sheet metal, probe along the base of the wall and try to insert the blade under the wa11. If you find places where the blade penetrates a few inches under the wall, it is highly probable that noise leakage occurs at these locations. Mark the locations and repeat the test on the other side of the wall. Similar penetration of the knife blade would be a positive indication of noise leakage, most likely caused by either warped flooring or wall base-plates. To eliminate such noise leaks you could remove the base board molding on both sides of the wall and lay a continuous bead of resilient type caulk between the bottom edge of the wall and the floor, on each side. The molding may be reinstalled over the caulking.

Sometimes you can find a noise leak simply by looking for a light leak. Ask a companion with a flashlight to direct a bright beam of light along the edges of the wa11, while you look for light leakage in a darkened room on the other side. A small hand mirror will be useful for looking into remote corners or otherwise inaccessible places. Detection of any light leakage signifies noise leakage. You might want to judge for yourself the effectiveness of this particular test. This can best be done at night. Close the door of a brightly lit room, such as a bathroom, and look at the door from an area in total darkness on the other side. If your door is installed in a typical manner, you might be surprised to see it framed in a corona or halo of light. The light coning through the edges of the closed door traces a path that sounds can travel just as easily. This demonstrates quite clearly why many bathrooms provide so little privacy. However, the absence of a light leak is not necessarily a sign of an effective acoustical seal, because sounds can travel along complicated paths and corners much too abrupt for light beams.

A test particularly useful for detection of air and noise leakage around windows and door frames is to use a hand-held hair dryer or a vacuum cleaner with a long narrow crevice tool fitted at the end of the hose. While your companion aims the air stream or suction at places where you suspect noise leaks, you explore for drafts on the oppcsite side of the wall. Wetting your hand or fingers will increase your sensitivity to the direction from which the draft is coming.

Another test to determine whether window or door gaskets are sealing properly, involves opening the unit, inserting a strip of paper against the gasket and closing the unit. If the paper is held tightly or you feel a noticeable amount of resistance as you try to withdraw it, the gasket is functioning properly at that point. This test must be repeated at intervals along the entire length of gasketing to determine the overall performance of the gasket. However, sound leaks that occur in double-door or double-window installations with two or more sets of gaskets may not always be detected by this method.

A more reliable test is to operate a noisy device such as a vacuum cleaner or a power drill in the offending room and listen on the other side of the partition wall for any noise leakage. Although you can use your ears to locate the noise leaks, you can make the job much easier by using a sound probe or detector. A very useful device is a stethoscope. The child's toy sold in some "educational kits" is quite adequate for this purpose. An arm's length of soft plastic or surgical tubing, one end of which can be inserted in your ear, will work nearly as well. In a pinch, you can use a cardboard tube. The center tube from a roll of wall paper or a mailing tube of about that diameter is about the right size to accomodate one ear, and is long enough to allow you to move about freely while you search for the noise leaks. With a sma11 lump of caulking compound, modeling clay, or 
putty at hand, you can make temporary sea1s of any leaks as you find them. This will make it easier to locate the major or dominant leaks that must be sealed before any appreciable reduction of the intruding noise can be expected. This principle applies to the radiation of noise from vibrating surfaces as well.

At times, the noise problem may involve the transmission of both airborne and structureborne sound through a building construction. In such cases, you have to determine which path is dominant before you can proceed to the next step with any hope of success. Remember that your ear, when pressed tightly against a vibrating surface, is a sensitive detector of vibration covering a fairly broad range of frequencies. Your finger tips are a sensitive detector of low-frequency vibration, when held lightly against a vibrating surface. However, because of its sensitivity and flexibility, the stethoscope is even better suited for detecting structural vibration and sound leaks. As you probe around the edges of a wall or door with a stethoscope, a noticeable increase in the noise level will signify a noise leak at that location. If you fail to find any leaks, press your stethoscope against the wall at several places. A substantial increase in the noise level upon contact with the wall surface will indicate that the noise is coming through the wall as structural vibration.

If there is no appreciable increase in the noise level, as detected by the stethoscope, and the intruding noise is still noticeable, then you have to examine other surfaces of the room. It may be possible that the noise is entering the room through the ceiling or the floor. The surface having the largest vibrating area or undergoing the most intense vibrational motion must be quieted before the reduction of noise from any other vibrating surfaces will have any significant effect.

A useful thing to remember while searching for sound leaks is that sound generally travels through a wall as easily from one direction as from the opposite one.
Since the sound path is reversible it may be convenient to interchange the positions of the noise source and the observer in dealing with noise intrusion problems.

If outdoor noise is entering your room through a leak in the exterior wall, it is sometimes easier to locate the leak by making noise inside the room, and detecting that noise on the outside. This arrangement, of course, causes less disturbance in your neightborhood. Whether the noise source should be placed indoors or outdoors will depend on the background noise levels in each location at the time of the investigation. The rule of thumb is to place the listener or investigator in the location with the lower background noise leve1, which generally, but not always, is indoors. In such cases, power mowers, chain saws, or motorcycles running at fast idle make excellent outdoor noise sources. For safety, such machinery should not be left running unattended.

Quite often, the manner in which a listener describes an irritating noise problem and the way it affects him might reveal the probable causes of the problem and ways of alleviating it. Table 7-1 gives a capsular description of some of the more common noise problems, their probable causes, and the acoustical measures needed to rectify them.

Table 7-2 outlines, in order of importance, the strategies and techniques that one could use in resolving a noise problem. The table also gives an estimate of the amount of noise reduction that one can expect from each technique. This order is chosen because noise from the major source must be attenuated before reduction of noise from subsidiary sources will have any appreciable effect.

For your convenience in solving specific noise problems, we have compiled in Table 7-3, at the end of this chapter, an alphabetized catalog of common noise sources, practical remedies, and references to other chapters in the Guide where more technical background information on noise abatement and control is presented. 
TABLE 7-1

COMMON NOISE COMPLAINTS, LIKELY CAUSES AND SOLUTIONS

Complaint

A. "This room is noisy"
Probable Causes

Noisy appliances, ventilation system

Room is excessively reverberant; if the sound of a person's handclap persists longer than a second

Outdoor noise intrusion

B. "It is difficult to concentrate"

c. "It's stuffy and oppressive in here"

D. "You can hear voices, but they are unintelligible"

E. "I hear whistling noise"
If conversation at an ordinary distance of 3-5 ft $(1-1.5 \mathrm{~m})$ is difficult, the ambient noise level is too high [greater than $70 \mathrm{~dB}(\mathrm{~A})]$. Excessive noise may be due to causes described above.

If there is adequate ventilation, the room is acoustically "dead." There is too much absorption, i.e., excessive drapery, rugs and pads and upholstered

furniture.

The sound transmission through a partition or ductwork, and ventilation noise in the mid-frequency range.

High-pitched sound usually is generated by ventilators and grilles; worn or defective washers, and valve seals in plumbing, heating and refrigerant systems. High-velocity gas flow through furnace burner jets or nozzles causes similar noises.

\section{Remedies}

Reduce noise output of source: install vibration mounts; isolate source in sound-insulating enclosure. Ventilation noise: reduce blower speed; install acoustic lining and flexible connectors in ducts.

Install sound absorbing materials, e.g., carpets and pads, drapery, upholstered furniture, acoustical ceiling. Total surface area of absorbent material should be at least one-fourth of total room surface area.

Install gaskets around existing windows and doors; install storm windows and doors; replace hollow core or paneled entrance doors with solid core doors.

If the remedies outlined above do not alleviate the problem, install a prefabricated, sound-insulated engineer's booth or field office enclosure.

Remove at least $50 \%$ of all sound absorbent material such as drapery, thick carpet and padding; or replace existing furnishings with lighterweight material.

Caulk or seal all visible cracks at ceiling and floor edges of party wall. Remove cover plates of all electrical outlets in party walls to check for back-to-back installation; in such cases pack cavities with glass fiber wadding and seal with a resilient caulk. If additional sound attenuation is required, acoustical modification of the party wall may be necessary (see text).

Set dampers at most quiet setting; place ear at grille, if noise is louder remove grille. If noise vanishes with grille removed, reduce blower speed or install new grille with larger and more streamlined openings and deflectors. Reduce pressure in plumbing system and isolate pipes and valves from supporting wall and floor structures with resilient sleeves or collars. Replace worn or defective faucet washers or valve seals. 
Complaint

F. "I hear my neighbor's TV and stereo"

G. "Footstep noises from the apartment above annoy me"

\section{Probable Causes}

Acoustically-weak partition wall due to inadequate construction, noise leakage through cracks at floor and ceiling edges or through back-to-back electrical outlets. Neighbor's TV set may be too close to party wall.

Rigid, light-frame construction of floor assembly; solid concrete floor slab covered with tile; lack of carpeting and padding.

\section{Remedies}

Use same methods as in $D$ above. Suggest that neighbor place resilient pads under his TV and stereo sets and relocate them away from the party wall.

Suggest that carpet and padding be installed on the floor above. If additional footstep isolation is desired, test both ceiling and the walls in your room with a stethescope to determine which is radiating most noise. If noise radiation from ceiling is greater, install a gypsum board ceiling mounted on resilient hangers, place fiber glass blanket in void between ceilings. In some cases, wall paneling mounted on resilient furring members may be required in addition. 
Procedure

Breakup

resonances.

Reduce flow

velocity to half.

Break pure tones into random spectrum, same power.

Reduce dominant frequency by a factor of two, as by halving fan speed.

Reduce radiating surface area by a factor of two. sound barrier by a factor of two.

Double distance from a source of sound.
Increase mass of

Reduction in Sound leve 1 , dB

$20 \mathrm{~dB}$ or more

$18 \mathrm{~dB}$

May be negligible as read on a sound level meter

$6 \mathrm{~dB}$

$6 d B$

$6 \mathrm{~dB}$

Up to $6 \mathrm{~dB}$

(small source), up to $3 \mathrm{~dB}$

Install soundabsorbent materials.

Increase dominant $\mathrm{Up}$ to $6 \mathrm{~dB}$. frequency by a factor of two.

Up to $6 \mathrm{~dB}$, mostly at high frequencies. (line source).

Estimated Decrease in. Loudness

Almost $75 \%$, possibly more. Resonances yield pure tones perceived as louder than mixed sounds of equal total intensity.

Almost $73 \%$

Depending upon composition, loudness may be reduced more than 50\%. (Equivalent to about $15 \mathrm{~dB}$ SPL.)

Lowered dominant pitch reduces loudness equivalent of additional $6 \mathrm{~dB}$ SPL. Total loudness can be reduced about $50 \%$.

Noticeable reduction in loudness (about 25\%).

Noticeable reduction in loudness (about 25\%).

Loudness reduction up to $25 \%$.

Noticeable reduction in echoes and high-frequency noise, usually noticeable effect on loudness.

Can reduce audibility for sounds if original is above $6 \mathrm{kHz}$. Higher-frequency sounds are more readily absorbed by air and weakened or deflected by barriers. A last resort.
Relative Reduction in Sound Power

$99 \%$ or more.

Almost $97 \%$

None

Reduces sound radiation efficiency by a factor of about four.

Reduces sound radiation efficiency by a factor of about four.

Reduces sound radiation efficiency by a factor of about four.

Reduces sound power to $1 / 4$, if sound source is small and in open space; to $1 / 2$ if long line source; by nil if large source or echoing space.

Usually reduces sound power to as little as $1 / 4$.

May be none. Likelihood of efficient sound radiation increased, but can reduce sound transmitted through enclosures or barriers by a factor of four. 
INDEXED CATALOG OF SPECIFIC NOISE PROBLEMS AND REMEDIES

AIR CONDITIONER, Central (Chapter 4)

Compressor: Select unit with low noise rating; mount on resilient pads preferably on a concrete block located away from bedroom area.

Grilles, Diffusers and Convectors:

Select streamline units with smooth, wide openings and low-spread discharge.

Fan: Choose slow-speed, large diameter units. Isolate fan housing from ductwork with flexible sleeves. Install fan on resilient mounts.

Motor: Choose slow-speed unit with symmetrical belt drive. Use resilient mounts.

Ducts: Treat ducts and dampers with sound-absorbent material; design ducts for large-volume, slow speed air-flow in preference to small volume, high-speed flow.

AIR CONDITIONER, Room or Window (Chapter 4)

Compressor: Select a unit with adequate power capacity to do the job.

Fan: Select a large diameter, squirrel-cage belt driven fan.

Gasket: Install perimeter gasket of soft rubber to isolate unit from wall or window structure.

Mounting: Mount on resilient pads.

Thermostat: Adjust unit to minimize starting and stopping noise which can be more troublesome than the noise of steady running.

Air Filter: If the air filter in the unit becomes clogged with dust, condensate may freeze on expander tubing and thus further restrict the air flow. This may cause whistling noise. Clean filters weekly during season to retain free air flow.

\section{AIRCRAFT NOISE}

Note alignment of main runways and flight patterns of commercial or military airports.
Methods of avoiding: (Chapter 5)

Recommended dwelling locations: (Chapter 6)

Sound insulation requirements of dwellings: (Chapter 5)

Recommended seat location for passenger: (Chapter 9)

\section{AIR HAMMERS}

Select units with exhaust silencer and sound insulating jacket. Choose minimum stroke needed to perform work. (Chapter 3)

\section{ALARMS}

Emergency vehicles (Chapter 2)

Noise levels: (Chapter 2)

Startle effects: (Chapter 2)

Traveling: (Chapter 2)

APPLIANCES (Chapters 3 and 4)

Choose units with low-noise rating where given.

Install properly: Use vibration mounts or resilient pads, flexible connectors, vibration damping coatings, and perimeter gaskets to isolate units from cabinet enclosures.

See entries under individual appliances by type.

\section{BIRDS (Chapter 5)}

Reducing noise intrusion: Caulk or gasket existing windows; install storm windows. This will also conserve energy by minimizing temperature variation in the home.

\section{BLENDERS}

Select unit with low-noise rating; (Chapter 4)

Container: select glass rather than plastic. 
Housing or base: choose heavy metal instead of light plastic bases.

Mounting: place on resilient pads.

Use as a vibration source for troubleshooting noise problems; (Chapter 7).

\section{BLOWERS}

Choose slow-speed, large-diameter fan with wide blades or squirrel-cage design; prefer belt-drive to direct motor drive. (Chapters 3 and 4)

\section{BOWLING ALLEY (Chapters 3 and 4)}

Reduce noise buildup and reverberation by sound-absorbent treatment of walls and ceiling. Avoid areas where conversation can be done only by shouting.

Alley must be vibration-isolated from building structure by means of resilient mounting.

BUSES

Avoid residences near major bus routes. Engines should be well tuned, well muffled. (Chapter 6)

Tire tread design should be selected for quiet running. (Chapter 9)

Brakes should be adjusted and properly maintained to avoid squealing.

Choose seats near center; (Chapter 9)

\section{CABINETS}

Choose solid-core, well-damped door. (Chapter 4)

Apply mastic to inner panels. (Chapters 3 and 4)

Tighten loose strike plates.

Line shelves and backs of cabinets with cork or rubber tile to absorb impact noise. (Chapter 4)

CAN OPENER

Place on soft rubber pad. Avoid mounting on walls. (Chapter 4)
CHAIN SAW

Select quiet unit with adequate power. (Chapters 3, 4, and 5)

Wear ear protection. (Chapter 2)

CHILDREN, VOICES AND PLAY NOISES (Chapters 4 and 5)

Avoid play in reverberant spaces; reduce noise buildup by sound-absorbent treatment of playrooms and classrooms, close off sleeping quarters with well-fitted windows, gasketed doors; treat ventilation ducts with sound-absorbing lining.

CLOCKS, Alarm (Chapter 4)

Give preference to the types of alarms that chime or turn on radios, if you live in a multiple dwelling. Place alarm clock on a soft padded surface. Be especially careful not to set the alarm on a surface which joins or touches the party wall between your apartment and the adjacent one, or on a wall adjoining another sleeping room.

\section{CLOTHES DRYER AND WASHER (Chapter 4)}

Select units with low-noise ratings. Vent clothes dryer where noise will give minimum disturbance. Isolate laundry room as much as possible from other living space.

Install units on resilient pads or mounts; use flexible connectors in water and electrical supply line; install air chambers in water supply line.

Use sound-absorbing and vibration damping materials on inside surfaces.

\section{COMBUSTION NOISE - FURNACE (Chapter 4)}

Have fuel-nozzle adjusted for minimum noise (this can be done with no loss of burning efficiency). Separate furnace room from rest of residence with fireproof partition, if possible. Air intake in most residential furnaces is direct from surrounding air space; replacement with duct from outdoors can reduce noise radiation to rest of house; it also might reduce need for humidifying heated air in the house.

Flexible connector and sound-absorbing 
TABLE 7-3 (Cont'd)

lining in air ducts can reduce transmission of combustion noise from the furnace to room areas.

\section{COMPACTOR}

Vibration isolate the unit from enclosing cabinet and floor by means of resilient mounts and gaskets. (Chapter 4)

\section{COMPRESSED AIR LINE}

Select quiet blower nozzle; install silencer on exhaust port; keep tight couplings. (Chapter 3)

Maintain uniform diameter; avoid abrupt changes in cross-section. Avoid rigid connection between pipe and wall, to prevent "sounding board" effect. (Chapter 3)

\section{COMPRESSORS}

Use muffler on air intake to reduce radiation of whistling noises. (Chapter 3)

Choose rotary pump rather than reciprocating pump; balance carefully. Vibration isolate unit from surrounding structures by resilient mounting; install flexible cunnectors in lines. Place unit in sound-insulated enclosure. (Chapter 3)

\section{CORRIDOR NOISE}

Treat corridor floor with carpeting and the ceiling with sound-absorbent acoustic tile. Install sound insulating doors with gaskets to reduce sound leakage into living areas. Walls of apartments that are common with corridor walls should be of soumd-insulating construction. (Chapter 4)

\section{COURTYARD NOISE}

Courtyards tend to be reverberant and noisy.

Seal windows that overlook courtyards and draw ventilation from other exposures. If necessary, increase weight of glazing in windowpanes or install storm windows. (Chapters 5 and 6)

Some reduction of noise level can be obtained in a large courtyard by planting trees and shrubs.
Avoid installation of air conditioners in areas with courtyard exposures.

\section{CREAKING}

Common causes of creaking in a house are loose wood joints. See entry under floor noises, also see Chapter 4.

For creaking door hinges and blinds, lubricate hinge pins. Weatherstripping improves the door seal and also restricts the freedom of the door to move and generate creaking sounds. Refit latches and locks.

Creaking of ductwork is caused by thermal expansion and contraction. Mount ductwork on flexible hangers. Flexible expansion joints may help relieve the problem.

\section{CROSSTALK}

Install acoustic barriers in ceiling plenums, crawl spaces, and ventilation ducts. (Chapter 4)

Flanking transmission and small acoustic leaks are common causes of crosstalk between supposedly isolated adjoining spaces. (Chapter 4) For general discussion of "Troubleshooting" and leak-finding techniques, see Chapter 7 .

\section{DAMPER NOISE}

Dampers are the vanes used to control the flow of air in ventilation and heating ducts. Damper noise is typically a whistling or hissing sound, and may be reduced by slowing air flow, see Chapter 4. If dampers are remotely located from the grilles, some improvement can be obtained by inserting acoustical lining or sound silencers in the intervening ductwork.

Some flutter noise may be generated in a flexible damper; substitution of more rigid, heavier, or streamlined dampers may help.

\section{DIFFUSER NOISE}

Diffuser outlets in ventilating systems frequently are sources of whistling or hissing noise. Reduction of air flow velocity is the most effective remedy. Replace existing diffuser with a large-diameter, low-spread, better-streamlined unit. In a pinch, 
TABLE 7-3 (Cont'd)

removing the diffuser altogether and replacing it with a "spreading plate", i.e., a panel treated with sound-absorbing material on the side toward the duct opening and suspended 4-6 inches below the opening can be used to diffuse the air flow quietly. (Chapter 4)

\section{DISHWASHER}

Generally, choose a dishwasher after a demonstration for quiet operation. Built-in dishwashers should be quieter because of the limited surface for radiating sound. However, rigid pipe coupling and drain coupling can transmit noise to the household plumbing. Install flexible connectors in pipe and drain lines to minimize vibration conduction. Load dishes so that they are not free to flap in the washing stream. Tighten loose fittings, especially door latches and shelf supports. Mount unit on resilient pads and isolate it from cabinet enclosure with rubber gaskets. (Chapter 4)

DOG BARKING (Chapters 5 and 10)

Voice your complaint to the owner of the dog. Dogs that bark persistently should not be left out in the yard at night, out of consideration for the neighbors. Keep your own dog indoors, he makes a fine burglar or fire alarm in your house.

Caulk or gasket windows; install storm windows.

\section{DOOR KNOCKER (Chapter 4)}

Replace device with a door chime. Door knockers that strike too heavily may be relieved by putting a small resilient felt or rubber pad at the point where it contacts the strike plate. The knocker itself may be replaced by a lighter-weight part.

\section{DOOR SLAMMING (Chapter 4)}

Install door closure dampers on a11 exterior doors or spring-loaded self-closing doors, such as screen doors. A resilient gasket or weatherstrip surrounding the door will reduce the impact noise, as well as help seal the door against acoustic leaks. Substituting a solid-core door for a hollow-core door may help to relieve door-slamming and door-rattling noise.

\section{DRAIN PIPES}

The principal generator of noise in drain pipes is turbulence from rapid and unsteady flow. The noise, however, generally becomes annoying because the drain pipe is fastened rigidly to some surfaces which act as sounding boards. Insertion of resilient pads in the pipe supports should reduce noise, as should spacing the pipe from floor supports by means of resilient padding. Where a choice is available, use the largest diameter of pipe, to maintain relatively slow, smooth flow; (Chapter 4). Use heavy-walled pipe such as ceramic or metal pipe rather than plastic pipe.

\section{DRILLS, PNEUMATIC}

Drills with exhaust silencers and sound-insulating jackets are available.

Repairs on city streets using pneumatic drills should not be carried out during sleeping hours; (Chapter 10). In many cities, ordinances forbid such noise during night hours.

Caulk or gasket existing windows facing source; install storm windows.

\section{DRUMMING, BELT (Chapter 3)}

Looseness makes belt drumming and slapping more likely. Adjust tension for minimum noise. If necessary, a damping idler wheel may be installed to maintain belt tension while reducing belt vibration.

\section{DUCT NOISE (Chapter 4)}

The principle causes of duct noise are creaking, rubbing and snapping of ductwork due to expansion and contraction. Turbulent flow through ducts causes roaring sounds.

Dampers in ducts cause whistling noise for high-speed flow, see entry under damper noise. Whistling noises generated at duct openings, branches or elbows can be transmitted through the ductwork. Sound conducted through the ductwork as though it were a speaking tube can be minimized by installing sound-absorbing material and sound baffles. 
Use of sound-absorbent lining, flexible connectors and stiffeners or bracing in ductwork generally alleviates such problems.

\section{ELECTRICAL EQUIPMENT (Chapters 3 and 4)}

Common sources of noise are loose laminations on transformers (which can sometimes be tightened), sparking (indicated by a hissing, frying sound), and, occasionally, "singing" of loosely-supported filaments in lamp bulbs. Sparking can be prevented by tightening electrical contacts, and by making sure that switch contacts are clean. Lamp bulbs that "sing" should be replaced. Loose laminations sometimes develop in the ballasts used with fluorescent lamps; these should be replaced, preferably with more conservatively-rated ballasts. Fluorescent lamps yield more light per watt of power than do incandescent lamps, but they tend to be noisier than the latter. Noisy wall switches that are likely to be turned on in the middle of the night - as in bathrooms can usually be replaced by "silent" mercury or rotary switches of the appropriate rating.

See individual entries for specific electrical appliances.

\section{ELECTRONIC AIR FILTERS}

Homeowners occasionally complain about the loud, sharp, snapping noises produced by the electronic air filter unit installed in their heating and air-conditioning system. Dust particles passing through the ionizing cell of the filter are given an intense electrical charge and are collected by highly-charged electrical plates. Abnormally large dust particles occasionally short across the electrical plates and cause arcing, which produces the objectionable snapping noise. The installation of acoustical lining or sound silencers in supply and return ducts for the purpose of attenuating mechanical equipment noise will alleviate the electronic filter noise problem as well.

\section{ELEVATORS (Chapter 6)}

An elevator shaft acts as a speaking tube to transmit sounds from the drive motors and elevator doors. Proper mounting of the motors and doors can reduce the noise produced. Sound-absorbing material can be installed in the elevator shaft, though this is seldom done. Bedrooms should not be adjacent to elevator shafts unless storage areas are placed in the intervening spaces.

Especially in tall buildings, where high-speed elevators are used, the air overpressure and rarefactions caused by the piston action of the elevator cab have to be vented somewhere; provision of alternative vents for this pressure may be feasible, and can prevent the whistling of air past the safety strips on elevator doors.

EXPANSION AND CONTRACTION (Chapters 3 and 4)

Long sections of sheet metal supported at widely separated points are likely to make "clacker" type noises as they expand and contract with temperature. This is a serious source of noise in ventilation ductwork. Separating the duct into segments which are supported independently and connected together with resilient or compliant joints can reduce sound from this cause considerably.

Sheet-metal roofing can be sealed with mastic placed at frequent intervals where it is laid on the roof. This can be used to minimize rattling in the wind. Careful attention to the edge of roofing can prevent the rattling (and tearing) produced when strong gusts of wind penetrate under the roofing and lift it.

In "vee-groove" roofing, nails should be applied through a spot of mastic applied at the spring section at the peak of the vee. Motion of the roof relative to the nail will then tend to clinch the roofing material around the nails.

FANS, EXHAUST (Chapters 3 and 4)

Remember the useful rhyming conditions "slow and low". Use as slow a fan speed as can be obtained. Exit louvers should be made of easily-cleanable material and massive enough to prevent rattling. In choosing a kitchen hood filter, give preference to one that is long, wide and made of thick highly porous material; this choice will tend to maintain smooth air flow. of 
course, for ease of cleaning the filter should be interposed between the hood and the fan.

\section{FAUCETS (Chapter 4)}

One of the greatest nuisances late at night is a dripping faucet with no immediate chance of fixing it. Simply placing a sponge or a facecloth under the drip may reduce the sound appreciably. The traditional quick remedy is to tie a string or shoelace to the faucet so that the drip is channeled as a miniature stream down the string. A more effective solution is to replace the worn washer that is causing the drip.

"Singing" of a faucet is almost always a sign of a deteriorating washer, which should be replaced.

\section{FLOOR NOISES (Chapter 4)}

Squeaking floors and stairways; Squeaking floors often can be silenced by inserting oil in grooves or edges of flooring, renailing, inserting wedges under warped board, installing additional support or bracing to sagging floors.

A curious slapping noise on tiled floors, such as parquet and the various linoleum, rubber and asphalt tiles, can be heard if a tile becomes loose. The loose unit can be located by walking briskly across the floor and noting which tile emits the sound. To prevent the tile or linoleum from cracking, apply heat from a hand-type hairdryer or pan of hot water to soften the surface. Even if partly secured, tiles may be lifted enough to allow mastic to be inserted under the loose edges. A weight placed over the tile will hold it in place until the mastic sets.

FURNACES (See entry under heating systems)

\section{GARAGE DOOR (Chapter 4)}

The noise generated by the opening and closing of roll-up type doors of garages attached to the home can be reduced substantially by mounting the track on resilient isolators and placing a rubber gasket at the bottom edge of the door to absorb the impact shock of closing the door.
GARBAGE COLLECTION (Chapters 4 and 5)

Vibration-damped, laminated metal trash cans, which are relatively quiet when handled, are commercially available. Cans made of heavy-gage, resilient plastic make less noise when dropped or struck than do ordinary metal cans.

If collection takes place at hours when it interferes with sleep, there may be a case for community action; (Chapter 10)

\section{GARBAGE DISPOSER (Chapter 4)}

If possible, select a unit for quiet operation. Such units feature resilient mounts, flexible pipe connectors and a sound-insulating outer shell. Coating the underside of the sink basin with vibration damping compound will improve the noise reduction. Installing acoustic tile inside the cabinet enclosure will reduce noise buildup.

GARDEN APPLIANCES (See entries under individual appliances by type)

The major noisemakers are lawn mowers and chain saws. Mowers can be selected for quiet operation. Reel mowers tend to be quieter (and often somewhat safer) than rotating-blade mowers. Ear protection should be worn if needed (Chapter 2) and attention should be paid to the community's needs for reasonable quiet.

Since chain saws are difficult to silence, they should be used only at times when neighbors will not be disturbed; adequate rest periods should be taken by the operator.

If it is possible to extend a nower cord to the working site, electrically-operated tools may eliminate a large degree of the noise.

\section{GRILLES}

The ventilator grilles on forced-air heating and cooling systems frequently are a major source of high-pitched noise. The most common cause of noise is high-velocity air flowing through the grilles; choice of a more streamline grille with larger openings will reduce the whistling noise. (Chapter 4) 


\section{GRINDERS}

Except when it is directly engaged in grinding, any rotary grinding tool should be so well-shielded and well balanced that it is essentially quiet. This can be improved by careful mounting of the grinder on resilient pads. (Chapter 3)

A portable grinder can be operated quietly when set upon a vibration-isolating mount. (Chapter 4)

HEATING SYSTEMS (Chapter 4, Control of heating, ventilating and air-conditioning system noise)

Most conventional heating systems generate noise, some more than others. For a given system, however, the amount of noise generated will depend upon the type of equipment used and method of installation.

One advantage of lowering the thermostat at night during the heating season is that it minimizes the number of times the disruptive cycling of the heating system goes into operation during sleeping hours. Flame noise can be minimized by proper choice of combustion nozzles and proper adjustment.

The quietest system is hot-water heat, if care is taken to vibration isolate the system from wall and floor structures by means of resilient mounts throughout the house. A noisy impeller can be replaced with a quieter one, or the rate of flow can be reduced, which will lower the noise output.

Steam systems generate noise through air vents, which whistle and hiss every time the steam is circulated; a two-pipe steam system is much to be preferred to the one-pipe steam system. Steam radiators also "hammer" if water gets trapped in the circulatory system, a particularly serious fault of the one-pipe steam systen. Place wedges under the legs of the radiator opposite the input end to increase the drainage angle. This will allow any trapped water to drain away and thus prevent water hammering.

Baseboard heating systems can cause annoyance, especially in the still of the night, if they are not installed properly. This applies to electrical as well as hot water systems. The major causes of the noises are the expansion and contraction of the heater assembly, component parts and piping, and the amplification of such noises by wall and floor structures in direct contact with the heating system. Noises from motors, pumps and turbulence in the case of hot water systems, and the low frequency resonance or huming associated with the electrical heaters also are sources of disturbance. Such problems can be alleviated by structurally isolating the heaters from walls and floors by using resilient spacers and leg mounts made of heat-resistant materials. Asbestos board backed with pads of silicone, neoprene or rubber would be suitable for this purpose, providing that the asbestos surface supports or is inserted next to the heater.

Many modern heating systems use forced air circulation because it permits air conditioning and humidification to be supplied through the same ducts. This is potentially one of the noisiest systems unless special care is taken in its design and installation. See entries under blowers, fans, ventilation, heating and air-conditioning equipment.

HEATING SYSTEMS, CENTRAL AND CLOSET INSTALLATIONS (Chapter 4)

Noise associated with forced-air heating systems is due to a combination of sources, such as mechanical noise of the motor/blower, blower-blade passage frequency, turbulence, air-flow noise, combustion or burner noise, thermal expansion and contraction of ductwork and pulsation and vibration of the ducts.

Substantial reductions in noise output can be made by designing systems for low-velocity and low-pressure operation. Placement of resilient mounts under the motor/blower and use of resilient hangers, flexible boots and acoustic lining in ducts will alleviate the noise problem.

Closet installations will require in addition soundproof walls, doors and an acoustic-lined return duct. 
Most household appliances have some magnetic parts in them, such as the armatures on motors and the reactors in fluorescent lamps. The magnetic sheets will vibrate against one another, and generate hum at multiples of the power line frequency. Where the frames are bolted, as in transformers, tightening the bolts may help to eliminate the hum. On reactors for fluorescent lights, replacement of the "potted" unit may be the only remedy available. Radiation of the hum by other parts of the fluorescent lamp may be decreased by resilient-mounting of the reactor ballast; (Chapters 3 and 4)

\section{HUMIDIFIERS}

The noise generally is caused by the fan or impeller. Atomizer-type humidification may generate a noise from the spray nozzle, which may be adjusted or replaced with a quieter unit. Since the spray noise is relatively high in pitch, acoustic baffles may help; (Chapter 4).

\section{HYDRAULIC CONTROLS}

The most common cause of trouble is valve noise, which is discussed under that entry. Also see Chapter 3.

As a rule, hydraulic and pneumatic controls are simple and rugged, but generally more noisy than electrical controls, with which they can sometimes be replaced. Sound insulating enclosures can be designed to suppress such noise.

\section{ICE CRUSHER}

See general discussion for kitchen appliances in Chapter 4.

Like a blender, it can be made quieter by using a heavy container for the crushing compartment, and mounting the crusher on a vibration isolator.

\section{IMPACT NOISE}

See Chapters 3 and 4 for general principles of reduction of impact noise in machinery, kitchen cabinets, and floors in homes and apartments.
These devices should be operated at times that do not interfere with sleep among the neighbors. Shock mounting helps. One should select an appliance that is designed for quiet operation. A hydraulic device can be made quiet more easily than can a device depending upon mechanical impacts to compress the trash. (Chapters 3 and 4)

\section{INCINERATOR CHUTES (Chapters 3 and 4)}

Frequently these are made of relatively thin sheet metal, which vibrate and rattle as the trash strikes against the flexible metal surface. Again, disposal of trash should be restricted during normal sleeping hours.

The metal chute should be structurally isolated from the building walls by means of resilient mounts. The exterior surfaces of the chute should be coated with a vibration damping compound to reduce the drumming resonance of the chutes caused by the impact of trash or refuse.

\section{JACK HAMMER}

A few models which feature a sound insulating jacket and exhaust silencers are available and should be used.

Workmen should wear hearing protection. Work with these tools should be confined to normal waking hours. Avoid long, continued use of the jack hammer by interposing adequate recovery periods. (Chapter 2)

Use of such noisy equipment may be a case for community regulation; (Chapter 10). Frequently, an additional source of noise is the compressor supplying air power to the jack hammer. Compressors with sound insulating enclosures are available and should be required.

See discussion under compressors and compressed air line in this index.

\section{KITCHEN APPLIANCES}

With the exception of washers and dryers, most kitchen appliances are used for brief periods of time. However, several appliances may be in use simultaneously such as clothes 
TABLE $7-3$ (Cont'd)

washer, dryer, dishwasher, garbage disposer, etc., which jointly can generate a rather high level of noise.

For methods of selecting and installing appliances for quiet operation, see Chapter 4.

\section{KNIFE SHARPENER}

Place the appliance on a soft resilient pad to isolate it from the kitchen counter top or working surface. (Chapter 4)

\section{LAUNDRY ROOMS}

The principal sources of noise are clothes dryers and clothes washers; refer to these entries. The rooms tend to have hard surfaces and are likely to be very reverberant. Noise buildup can be reduced by installing sound absorbing material such as perforated metal tiles backed with glass fiber pads, which will withstand the moisture. Resilient mounts and flexible connectors should be used on all machines; (Chapters 4 and 6).

For gas-operated dryers and water heaters, consider using an exterior-opening duct to draw outside air for combustion. This will reduce the level of combustion and burner noise radiated indoors.

\section{LAWN EDGERS}

These tend to be as noisy as gasoline-powered lawnmowers but have a high-speed blade, which emits a somewhat louder and more irritating high-pitched noise. Some models are noisier than others. Be selective in choosing a quiet unit. Ear plugs should be worn; they are especially effective against high-pitched noise.

\section{LAWN MOWERS}

Rotary Power Type: Select model with low noise rating, preferably with under-deck exhaust. Replace the conventional muffler with a new, somewhat more expensive, but highly effective model. Sharpen cutting edges of the blade and balance the blade. Tune engine and reduce speed; tighten loose and rattling parts. The above measures should provide about a $6-d B$ or about a 25 percent reduction in loudness. Generally speaking, noise levels at the user's position frequently exceed $90 \mathrm{~dB}(\mathrm{~A})$, although this does not pose a hearing hazard because of the brief use of the mower, once every week or two. However, it may prove very hazardous to workers whose daily occupation is the mowing of lawns, park lands or the grounds of large institutions. Parents should consider this point very carefully before allowing their children to mow lawns to earn money on weekends or during summer vacations. In any event, ear protection should be worn by all workers exposed to such noise for extended periods. Refer to Chapter 2 for a discussion regarding noise-induced hearing damage. Reel-type power mowers, because of their smaller radiating surface, are somewhat less noisy than the rotary type. Properly designed electric-motor type mowers generally are the least noisy. Mower manufacturers can make substantial reductions in the noise out of their machines by adopting the following recommendations.

(a) Install the most effective, not the lightest, muffler available.

(b) Vibration isolate engine from the mower deck.

(c) Except for the cutting edge, the blade should have rounded corners and a serrated or feathered trailing edge.

(d) Underdeck should have smooth streamline air flow passageways devoid of any obstacles or sharp corners.

(e) Construct the deck of laminated sheet metal with a viscoelastic core to suppress deck vibration.

(f) Enclose engine compartment in a sound-insulating jacket or cover.

\section{LAWN THATCHERS}

Fortunately, these are used infrequently on any given lawn. The noise problems are common to those of lawn mowers and edgers, i.e., the engine is the main source of noise. Select units with well-muffled engines. The operator should wear ear protection. See related entries. 


\section{LEAF BLOWERS}

Choose a model with a well-muffled engine, large air-inlet aperture, a squirrel-cage blower, and a streamlined discharge nozzle. Select attachment hoses that have smooth inner surfaces. Operate blower at slowest speed that will perform the chore.

\section{LEAF SWEEPERS AND MULCHERS}

Select machinery with well-muffled engines. Leaf sweepers are available in two types - the vacuum and the rotating brush types. Requirements for quiet operation of the vacuum-type are similar to those for a leaf blower. Canvas hoppers should be selected rather than metal to absorb the noise produced by the impacts of stones, acorns or debris. Metal hoppers should be coated with a vibration damping compound. The cutting and blowing noise of a mulcher also can be reduced somewhat by coating the inside surfaces of the metal housing with vibration damping material.

\section{LIGHT SWITCHES}

Silent mercury-type light switches and thermostats are available. Rotary-disc type switches also are relatively quiet in operation compared to the spring-operated type switches.

\section{MASKING NOISE}

See discussion under "Is There An Acoustical Perfume?" at end of Chapter 3 .

\section{MECHANICAL EQUIPMENT}

See individual entries in this section and in Chapter 3 for methods of reducing the noise output of various types of machinery and mechanical equipment.

\section{MOTORS}

On electrical motors, eliminate unbalanced loads and replace worn bearings to prevent vibration and thumping noises; oil dry bearings to eliminate screech.

Motors should be installed on resilient mounts and vibration isolators.
Stray electromagnetic field from motors can set steel housings into vibration; replacing steel housings by brass or other non-magnetic material may alleviate this noise problem.

\section{MOTORBOATS}

Relatively quiet outboard motors are available. They come equipped with vibration isolating mounts, sound insulating jackets, and under-water exhaust systems. Inboard motors with basically the same features can be installed in an acoustically-lined, sound-insulated enclosure to provide a greater amount of noise reduction. Electrical, battery-powered motors which operate quietly are available for light use, such as trolling or fishing. In addition they do not pollute the water.

\section{MOTORCYCLES}

Quiet mufflers are available. Except for delayed adolescence, there is no reason to use a poorly-muffled vehicle. With a properly designed muffler, power from the engine will be more smoothly developed, and greater fuel efficiency can be expected.

\section{MUFFLERS}

In general, mufflers are sound-absorbing chambers placed on the exhaust of internal combustion engines to prevent the discharge of noise from the combustion process. As a temporary measure, additional muffler action can be improvised by lining a large-diameter metal can with a pad of glass fiber and inserting a slotted, smaller-diameter can. This double wall muffler should then be attached to the end of the exhaust pipe.

To work properly, a muffler must be designed for a given noise spectrum, sealed carefully to the exhaust pipe, and must itself have substantial, non-rattling walls, and sound baffles.

MUSIC

Unwanted music becomes noise. Two rival sources of music generate cacophony. Rock bands approach sound levels hazardolis to hearing. (Chapter 2) Similar levels can occur with some "Hi-Fi" systems. 
Avoid places that feature such loud music. Warn your children about the hearing hazard. See Chapter 4, Recreation Rooms, for methods of sound-proofing rooms against loud noise or music.

NAIL GUNS

Relatively quiet models are now commercially available. Avoid prolonged use of the device on a daily basis unless hearing protection is worn.

\section{NEEDLE VALVES}

The characteristic noise emitted by a needle valve is a high-pitched hiss. Examine valve and replace it if it is defective. Vibration isolate valve and associated piping from large radiating surfaces by means of resilient pads or sleeves. Enclose valve in glass fiber padding with an outer jacket of leaded viny 1.

\section{OFFICE EQUIPMENT}

See Chapter 8 for methods of quieting office machines and equipment.

OUTBOARD MOTORS

See discussion under MOTORBOATS.

\section{PIPES}

See Chapter 3 for design of quiet flow systems, and Chapter 4 for control of plumbing noise.

PLUMBING NOISE

See Chapter 4, Control of Plumbing Noise.

\section{POLISHERS}

Quiet models are available. These feature slow-speed, large diameter rotating discs which can be fitted with brushes or buffing pads.

\section{POWER TOOLS}

Variable speed tools like drills and scroll saws are less noisy than the high speed/torque models. Operate the tool at lowest speed capable of doing the job. See entries under specific type of tool.
Sump pumps and automatic-leveling water pumps are serious noise offenders, particularly if a large quantity of water is drawn late at night. Vibration isolate the pump from the building and connect it to the plumbing via flexible tubing. Seal connectors carefully for maximum pumping efficiency (briefest operating time).

Pumps for compressed air systems are exceptionally noisy devices. See entries under compressors and jack hammers.

See Chapter 3, Section 5 and Chapter 4, Control of Plumbing Noise.

\section{RADIATORS}

Steam radiators are inherently noisier than hot-water radiators; one-pipe steam systems are noisiest because of air-venting requirements and the frequent onset of vapor lock (water condensed from cool steam blocks the access of new steam in the pipes which causes intermittent hammering). Select a hot-water system which features large diameter pipes and low velocity flow. Vibration isolate the impeller pump from the building structure. Separate the pump from the pipe system by means of flexible connectors. "Bleed" dissolved air out of hot-water system at regular intervals to avoid hammering sounds from trapped air bubbles. See Chapter 4 relative to steam and hot water heating systems.

RADIO

Adjust volume control of the radio so that the sound level of commercials (which usually are about $5 \mathrm{~dB}$ higher than program material) is at a comfortable conversational level.

\section{RAILROAD}

A railroad is a source of vibration as well as airborne noise. Select dwellings that are at least one mile, preferably two miles, from a railroad line. See Chapter 6, "Selecting a quiet home or apartment site."

If you live near a railroad see Chapter 5 for recommendations regarding insulating your house against outdoor noise. 


\section{RAIN GUTTERS AND SPOUTS}

A major noise source is the turbulent flow and irritating dripping of rainwater in the gutters and downs pouts. (Chapter 4)

Select rain gutters of heavy gage metal, rounded bends and coated with vibration damping material. Avoid installing vertical drains outside of bedroom areas; isolate drains from the building structure by using soft rubber sleeves at pipe clamps and gutter spouts. Replace conventional metal elbow at base of downspout with a soft rubber boot or plastic hose.

\section{REFRIGERATORS}

Select units for silent operation. Frost-free refrigerators are operated with numerous fans, all more-or-less contributing to a noisy kitchen. Any refrigerator will be less noisy if it is mounted on resilient pads on a solid floor. (Chapter 4, Kitchen Noise, item 12)

\section{RISERS}

Vertical ducts or ventilation risers mounted on the exterior of buildings frequently are the cause of noise complaints. Such devices often rattle in windy areas or snap, crackle and pop, owing to thermal expansion and contraction with outdoor temperature variation. Further, the outdoor noise of aircraft, traffic, etc., are easily transmitted by the thin-wall duct and carried into the building interior. All exterior ductwork should be of double-wall construction with acoustic lining and silencers. Risers should be vibration isolated from exterior walls by means of resilient mounts at all points of support.

ROCK AND ROLL MUSIC

Just possibly, this term may become obsolete before this Guide is published, but anyone who has been exposed to amplified music has heard the noisiest part of "Rock and Roll." The sound levels generated can be high enough to damage the hearing of audience, performers and bystanders. (Chapter 2)

If you must have a rock and roll session at your house, and if you must play the music so loud that you experience the pleasurably giddy sensation that high sound levels seem to produce in some people, the only way to avert noise complaints is to give a block party and invite all the neighbors in.

Obviously, shutting all windows and doors will reduce the amount of noise escaping outdoors.

\section{ROOF NOISE}

"Rain on a tin roof" is a proverbial source of noise. Metal roofing can be quieted to some extent by mounting it over a layer of mastic. Slate roofing is strong and relatively quiet, though liable to breakage by hail and repeated freezing and thawing, but can only be applied to a roof structure capable of withstanding its great weight. Asbestos roofing is quietest, but most likely to tear in high winds. Massive roof materials like lead, copper, and slate serve best as barriers against exterior noise; sound insulation of the roof of a house is just as important as it is for the walls. Also see entries under expansion and contraction.

\section{ROTOR TILLERS}

The noise generated by a rotor tiller is comparable to that from a power lawn mower; see entries under LAWN MOWERS.

\section{SANDERS}

Since most noise is generated at the contact between the sander disc or belt and the work, little can be done to prevent noise from being produced. At the factory wear ear protection. Provide for adequate rest periods away from the noise. Work in well-ventilated areas, preferably treated with sound-absorbing baffles or acoustic tile ceilings.

\section{SAWS: BAND, CHAIN AND CIRCULAR}

Noise reduction at the source is difficult. In the case of chain saws, a good muffler can make a substantial reduction in noise output.

Wear ear protection and provide for adequate rest periods away from the noise during the working day. 


\section{SEWING MACHINES}

The reciprocating motion of the machine generates considerable impact noise. Place rubber pads under the legs of the machine cabinet or under the machine itself if it is a portable tabletop model. Resilient mounting of the machine in the cabinet will result in a noticeable reduction of noise.

SHAVERS, ELECTRICAL

Motor-driven models are less noisy than vibrator types.

\section{SHOWER STALLS}

Shower stall should be mounted on a resilient pad or underlayment. Plumbing fixtures and drains should likewise be resiliently mounted. Metal or fiber glass stalls should be coated with vibration damping materials. See Chapter 4 on control of plumbing noise and Chapter 3 on vibration damping materials.

\section{SIRENS}

Audibility is a natural requirement, useful except for those who live near an emergency facility such as a fire station or hospital. Fortunately, the spectral distribution of sirens clusters within the $500-2000 \mathrm{~Hz}$ frequency range, which can be excluded to a considerable degree by closing off living quarters from outside noise, just as one tries to do for highway noise. See Chapter 5 for reducing the intrusion of outdoor noise into the home. See Chapter 2 relative to detection of sirens.

\section{SNOWMOBILES}

Many communities are beginning to regulate the noise emission that will be tolerated from snow mobiles and other all-terrain vehicles.

Select machine on basis of low noise output. Such machines feature effective mufflers, acoustical lining, and vibration damping treatment. As a precautionary measure, ear protection should be worn, particularly during periods of extended or prolonged use.

\section{SOLENOIDS}

Many automatic household machines such as dishwashers and washing machines use electrically-operated solenoids to open and close input and drain valves. The basic mechanism is a rod of magnetic material operated by a coil. Usually, a fairly heavy impact occurs when the solenoid is actuated. Shock-mounting the device may help to confine the noise to the machine and its immediate vicinity. Sometimes, a solenoid held back by a spring will vibrate when the slot in which it travels becomes worn, and replacement will cut down the hum level significantly.

\section{SONIC BOOMS, SHOCK WAVES}

\section{Also see entries under THUNDER.}

Depending on the distances involved, by the time a sonic boom or shock wave reaches your house, it usually will have lost some energy by spreading, air absorption, and perhaps reflection off natural or man-made barriers.

Building structures that are adequately sound-insulated against aircraft or traffic noise will also do well against sonic booms; but due to their suddenness and infrequency, sonic booms produce startle and short-term interference that are more like that caused by aircraft flyover noise. Community action may be required to resolve the problem of sonic boom disturbances caused by supersonic aircraft flyovers and the shock waves caused by "blade slap" associated with helicopter flights. (Chapter 9)

\section{STAIR CASES}

The impact from footsteps can be minimized by placing carpets or resilient pads on the treads. Stair halls are often needlessly "live", and can be quieted considerably by the use of fireproof, sound-absorbing material on the upper walls. (Chapter 4).

In apartment buildings, staircases that are sealed off with fire-proof doors equipped with quiet closures minimize not only the fire hazard but, also noise interference.

\section{STEAM AND PRESSURE-REDUCING VALVES}

Such valves are exceedingly noisy. Replacing existing valve with a number of smaller units to effect a gradual reduction in pressure will lower noise 
output considerably. Enclosing valves in a gypsum board or plywood box, or duct packed with fiber glass is an effective means of reducing the noise level.

\section{STEREO SETS}

See entries under MUSIC.

\section{SUMP PUMPS}

See discussion under PUMPS.

\section{SWIMMING POOLS}

The hard tile surfaces surrounding an indoor swimming pool and the water surface of the pool cause the area to be highly reverberant and thus build up the noise made by persons at play within the pool. Various types of sound absorbers that are moisture resistant can be used within the pool area to reduce the noise level, but seldom are.

Pumps and circulation systems which are additional sources of noise should be structurally isolated by means of resilient mounting and flexible connectors to minimize noise buildup. A rooftop swimming pool is a nearly intolerable source of noise to the residents beneath. Such installations require vibration isolation of the pool, and the plumbing and filtration system from the building structure.

\section{TELEPHONE}

If you are disturbed by the noise of a ringing telephone, select desk type telephones in preference to wall-mounted models. Owing to the sounding board action of the wall, the wall-mounted telephone tends to be considerably noisier than the desk type unit. For this reason, one should refrain from having wall-mounted telephones installed in bedroom areas or on party walls separating dwelling units.

Most telephones are equipped with a volume control to adjust the noise level of the bell. Place phone on soft rubber pad.

If you need only to know when there is a call for you, and don't wish to have an audible signal, you can arrange to have the ringing signal replaced by a flashing light. This is done routinely by the telephone company for hard-of-hearing clients, and is available in most systems.

If you are hard-of-hearing, and the telephone is in a noisy environment, you can improve your chances of hearing the conversation by having your hearing aid fitted with a telephone pickup coil, which picks up the electrical signal from the earphone of the telephone. This will reduce markedly the competition from the room noise.

\section{TELEVISION SETS}

Noise complaints involving television sets are like those concerning "Hi-Fi" sound systems and rock bands; the sound levels at which they are played are objectionable to the neighbors. In apartment buildings, TV noise disturbance can be reduced by requesting tenants to locate their sets away from party walls, place resilient pads under the legs of the cabinet or stand, and set the volume control at a lower level.

Another source of objectionable noise is the high-pitched tone radiated by a poorly-adjusted TV set. Persons with normal hearing can find this noise quite bothersome; however, it can be removed by having the set readjusted by a repair man.

Occasionally, a high-pitched noise is radiated from a pulse transformer which is usually located in the rear of a television set. The noise, which generally is directional, can often be reduced by installing fireproof acoustical tile on the wall directly behind the TV set.

\section{TELEVISION ANTENNA}

The television antenna acting like a vibrating reed can generate a moaning or mournful sound on windy days, if it is mounted on the roof or attached to the chimney of a house. This problem can be corrected by bracing the antenna with guy wires or tie rods, or by installing the antenna in the attic area where it would be sheltered from the wind.

\section{THERMOSTATS}

See discussion under LIGHT SWITCHES. 
THUNDER

TABLE $7-3$ (Cont' $d$ )

The noise from thunder is very low-pitched if the thunder arises from a lightning stroke at a fair distance from the listener. Methods used for excluding traffic noise, such as solid walls and well-sealed windows and massive roof structures also will reduce the intensity of the sound from thunder inside your house.

\section{TIRE NOISE}

See Chapter 9, Automobiles.

\section{TOILETS}

See Chapter 4 on control of plumbing noise.

Water flow in the drains is a major source of noise. "Silent flushing" toilets are available.

Syphon-jet toilets with flush-tank fixtures equipped with adjustable flow valves are considerably less noisy than conventional models. A high-pitched noise which occurs when the flush tank is being refilled usually is a sign of defective seals. Replacement of the seals eliminates the problem.

TOOLS

See entries for individual tools such as Saws, Sanders, Grinders, etc.

TOYS

Toys perform useful functions for children, but their use does not justify risking a child's hearing.

Explosive devices such as cap and air pistols are a serious hazard, now subject to regulation in many states (see Chapters 2 and 10).

The regulations, unfortunately, do not protect against the use of noisy toys in reverberant areas, rooms with hard, reflecting surfaces that will permit the noise to build up. It is a good idea to restrict the use of noisy toys to out-of-doors, where reverberation and noise buildup are less likely to occur.

Musical instruments, such as trumpets, should not be put into the hands of children who might carelessly blow them near another child's ear.

Noisy firecrackers are banned in many places, primarily as a fire and explosion hazard, but occasionally a child may be deafened permanently by another child's prank. This loss, though less visible, is a very serious problem.

\section{TRANSFORMERS}

A transformer carrying a fairly large amount of power can be a serious noise problem. The core of the transformer is made up of layers of magnetic iron a1loys. Occasionally these layers ("Iaminations") work loose and vibrate with the frequency of the alternating current passing through the transformer. On large transformers, there is often a provision for tightening the bolts holding the laminations together. On cheaper appliances, such as the fluorescent lamps and high-intensity lamps, the laminations are "potted" in a tarry mixture, which may become heated and leak out, permitting the laminations to vibrate. These must be replaced.

\section{TRAFFIC NOISE}

For methods of reducing the intrusion of traffic noise into your living quarters, see Chapter 5.

\section{TRAIN NOISE}

Airborne noise from a train can be treated like traffic noise. (Chapters 5 and 6). Also, see entry under RAILROAD.

For selection of quiet accomodations while travelling in trains, see Chapter 9.

\section{TRUCK NOISE}

For control of tire noise, see Chapter 9.

For protection of dwellings from truck noise, see Chapters 5 and 6 .

\section{VACUUM CLEANERS}

Relatively quiet models are now available. Such cleaners feature streamline blower and air passage design, vibration isolation of the motor from the housing, and use of 
sound absorbent and vibration damping materials within the housing or canister. In canister-type cleaners, replacing corrugated hoses with smooth-wall hoses may eliminate pure-tone whistling noises caused by air-flow turbulence in the hose.

\section{VALVES}

Quiet valves are now available. Since a valve involves the constriction of fluid flow, it is always a potential source of noise.

A smooth flow path for the fluid should be provided at the entrance and exits of the valve. See Chapter 3 for design of quiet flow systems. A valve that gradually becomes noisier generally has worn gaskets, seals or valve seats. Replacement of the defective part usually alleviates the noise problem.

Reduction of water or fluid pressure frequently lowers the noise output substantially.

To prevent valve noise from being carried all over the house, consider the following measures: isolate the valve from the rest of the system by mechanically flexible couplings; avoid rigid clamping of plumbing systems to such sounding boards as floors and walls; run the piping through sound-treated ducts.

See Chapter 4, Control of Plumbing Noise.

\section{VEHICLE NOISE}

See entries under Traffic Noise and under individual types of vehicles, such as trucks, trains, etc.

\section{VENTILATION SYSTEMS}

See Chapter 4, Control of Heating, Ventilating, and Air-Conditioning Noise.

\section{VIBRATION}

See discussions in Chapters 3 and 4 relative to vibration isolation and control of structure-borne noise.

WALLS

See Chapter 4, Improving the Sound Insulation of Walls.

\section{WATER COOLERS}

These have major noise sources in the refrigeration equipment and in the plumbing connections. See entries under Compressor, Plumbing Noise, Refrigerators, and Valves.

See Chapter 4, Quieting of Large Appliances.

WASHING MACHINES

See entries under Plumbing Noise, Valves, Solenoids, and Drain Pipes. See Chapter 4, Quieting of Large Appliances.

\section{WATER NOISES}

See Chapter 4, Control of Plumbing Noise.

\section{WATER HAMMER}

See Chapter 4, Control of Plumbing Noise.

Water is a heavy fluid; when its flow is suddenly interrupted, its inertia will produce a sharp rise in pressure at the shutoff valve. Sudden starting or stopping of the flow of water gives rise to "water hammer", unless some means of prevention is applied. In plumbing systems, an air cushion is used to absorb some of the shock. A defective washer in a tap, by producing a sharply intermittent flow of water, can be a potent source of water hammer. This can be easily remedied by putting in a new and better fitting washer. Gate valves are more likely to produce water hammer than are needle and globe valves.

Hammering can also occur when piping systems are loosely supported on " $V$ " shaped wire hangers. Placing a rubber sleeve around the pipe and clamping it to. some solid structure will resolve the problem.

\section{WHISTLING NOISES}

Turbulence generated by high-speed air flow past the edge of an obstacle produces a whistling tone.

Such noises often occur in ducts, dampers, ventilation grilles, on the blades of fans, and usually around 
obstacles in the air stream that have sharp or ragged edges or narrow restrictions.

Turbulence is strongly dependent on flow velocity. A slight reduction in flow velocity will often result in a noticeable decrease in noise radiation, particularly in the high frequency range.

See Chapter 3 on design of quiet flow systems and Chapter 4, Control of Plumbing Noise.

\section{WINDOW RATTLE}

If window panes rattle, look for breaks in the putty. If the entire frame rattles, check the adjustment of springs or weatherstripping. Double-hung windows in aluminum guides can have the guide spacings adjusted for good fit. Wind noise and rattling in casement windows are more difficult to prevent because such windows are harder to seal or weatherstrip properly.

In a pinch, you can make a temporary stop to window rattling by forcing a wedge of wood between the panel and the window frame, or by wedging the space between the panels of double-hung windows. A more satisfactory and durable repair is to refit the window sashes and install adequate weatherstripping.

Storm windows provide acoustic insulation as well as thermal insulation and in the interest of quiet, can be left on windows that need not be open. 
NOISE CONTROL AT THE OFFICE AND SCHOOL

Noises at the office often can reach levels high enough to interfere with a person's ability to do his work. Interference in communication, disruption in concentration, and invasion of privacy are among the chief complaints.

\section{NOISE SOURCES}

The main sources of noise in most offices are:

(a) conversation among workers and on the telephone,

(b) noise from typewriters,

(c) ringing telephones,

(d) noise from office machinery, such as copiers, teletypers, etc.,

(e) noise from ventilation systems, and

(f) intrusion of outdoor noise, particularly traffic and aircraft noise.

\section{REDUCING THE NOISE}

In order to control the noise the first thing to do is to examine the office enviromment. If the wall, ceiling, and floor surfaces are hard and smooth the space will be excessively reverberant and will tend to amplify the noise to disturbing levels. Installing an acoustical ceiling, draperies, and thick carpeting will lower the noise level approximately 5 to $8 \mathrm{~dB}$ (or approximately $1 / 4$ to $1 / 2$ in loudness).

Typewriter noise may be reduced by placing soft rubber pads under the machine to prevent vibration of the desk top. Applying vibration damping material to the underside of the desk top or typewriter stand, particularly if it is made of metal, will tend to reduce the sounding board effect and the usual noise buildup.

The noise of ringing telephones may be reduced almost to inaudibility. There is a control in the base of the telephone that adjusts the ringing volume. The control can be reset to lower this volume. In fact, the bells can be replaced with flashing lights in most instances. office equipment like copiers, teletypes, computers, card punches and printers often are excessively noisy. If at all possible, such equipment should be installed in a separate room, such as a storage area. Otherwise, it should be located in an area as far as possible from office personnel. Acoustically lined barriers or partial enclosures should be placed around the machines to confine the noise. Any other noise control measures usually require making modifications to the equipment itself, as illustrated in Fig. 8-1. This should be done when the machine is idle or awaiting repairs, and preferably by a skilled technician or repair man. In most cases, the machine noise can be reduced by adopting the following recommendations in the order listed. This order is chosen on the basis that noise from the major source must be attenuated before reduction of the noise from subsidiary sources will have any significant effect.

1. Cover all ports or openings around card punches, copiers, readers and printers with $1 / 4$ inch (6 mm) thick clear plastic shields edged with soft rubber gaskets to make an airtight seal.

2. Wherever possible, vibration-mount all drive motors and cooling fans on rubber pads, or use rubber grommet-type sleeves on mounting bolts.

3. Treat all console panels with viscoelastic-type (e.g., gum mastic) vibration damping material.

4. Install $3 / 4$ inch $(19 \mathrm{~mm})$ thick fiberglass board lining on the inside surfaces of console paneling to reduce the noise build-up in the hollow reverberant cavities.

5. Instal1 acoustically lined ducts at intake and discharge sides of cooling fans.

6. Install resilient pads or vibration isolators under all equipment to reduce low frequency vibratory transmission to the supporting false floor. 


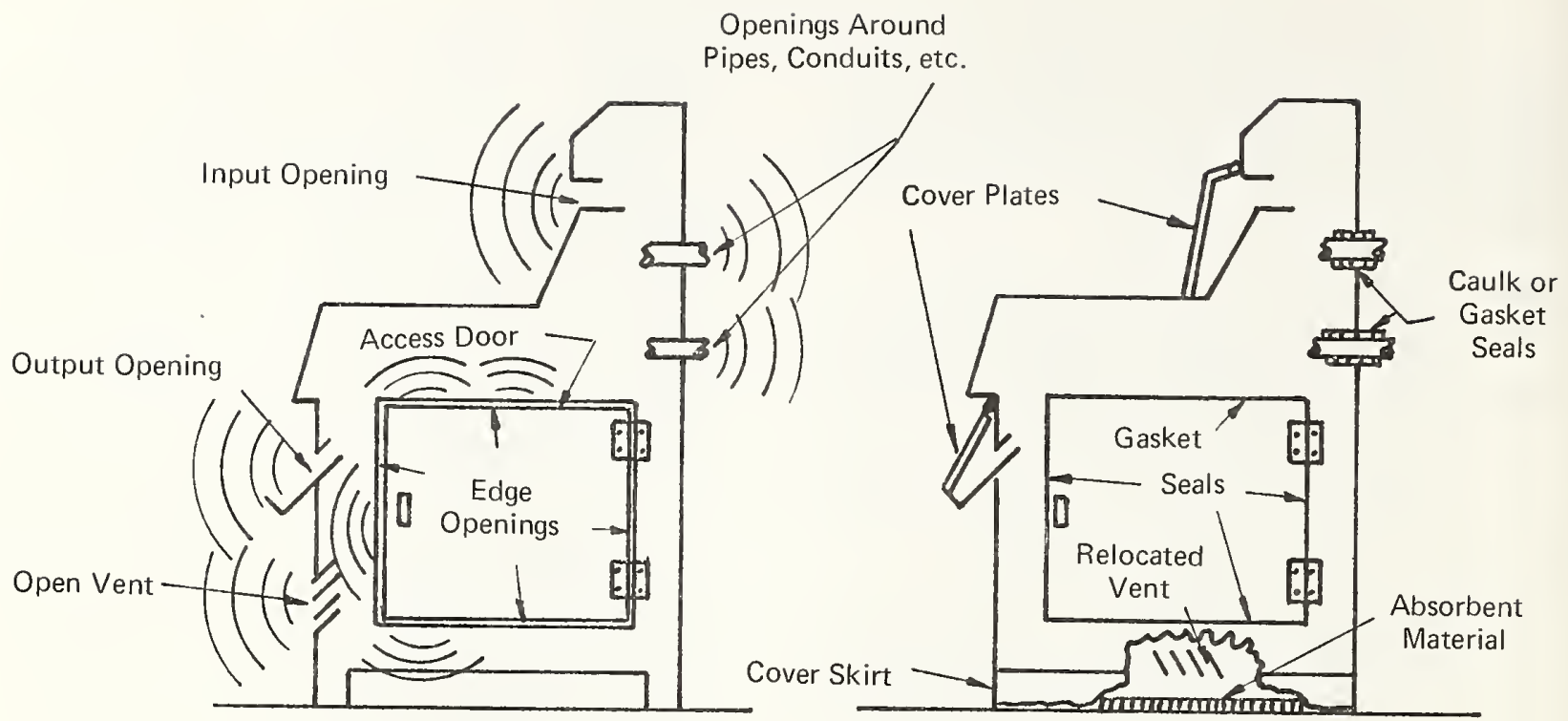

Fig. 8-1. MINOR DESIGN CHANGES FOR QUIETING OF OFFICE MACHINES

7. Install acoustically-lined U-shaped partial enclosures around individual card punches, readers and printers.

8. If a choice is available, give preference to large diameter slow speed fans and wide ducts in providing for the cooling of equipment.

The above measures should be attempted a step at a time in the order listed until the noise level in the office is reduced to acceptable limits. However, the most effective way of quieting office equipment is to incorporate good noise control techniques early in the design stage. This, of course, is the responsibility of the design engineer. However, as in the case of purchasing home appliances, the buyer can encourage manufacturers to adopt such practices by giving preference to quiet equipment.

\section{EXTRANEOUS NOISE}

The intrusion of noise from outside sources such as automotive traffic or aircraft flyovers can be minimized by improving the sound insulation of the windows, which usually are the weakest acoustical barrier against outdoor noise. In buildings that are air-conditioned, you should seal existing windows tightly with non-hardening caulk; if additional sound reduction is required, you should install storm windows, preferably encased in rubber gaskets. See Chapter 5 for more detailed instructions for minimizing the intrusion of outdoor noise.

\section{PRIVACY BETWEEN OFFICES}

If lack of privacy between offices, as for example, between an executive's office and the outer office, is of major concern, you should examine walls, doors, and ventilation ducts separating the two spaces to determine their sound insulating capabilities as well as the presence of noise leaks. In most cases, the door separating the office areas is the weakest barrier against indoor noise transmission. More than likely it is a hollow core door, constructed of thin veneer panels and with an open air space of $1 / 2$ inch $(13 \mathrm{~mm})$ or more at the base. Such a door at best will provide a noise reduction of only 10 to 15 $\mathrm{dB}$. Replacing the door with a solid core, soundproof door with gasket seals at the 
top and sides and a threshold seal will correct the problem.

However, if the partition wall separating the two offices extends only to the underside of a suspended ceiling, noise from one office can easily travel through the ceiling plenum over the walls and emerge into the space on the other side, as shown in Fig. 4-16. Measures to correct this problem involve installing an acoustical barrier of lead sheet and fiber glass blanket in the plenum space immediately above the wall. Noise leakage at the base of the wall can be controlled by caulking the bottom edge on both sides of the wall with a resilient, non-setting compound.

Ventilation ducts serving both spaces should be acoustically lined or equipped with baffles to prevent them from acting as speaking tubes. If additional sound insulation is required after the above measures have been adopted, then an additional wall or resilient attachment of a layer of gypsum board to the existing wall will be required. This would involve attaching wood furring strips to the existing wall, nailing resilient metal channels horizontally across the furring strips, and fastening the wallboard to the channels with self-tapping screws, as illustrated in Fig. 4-17.

A relatively simple and moderately effective measure for improving the privacy between two offices is to locate personnel, desks, office machines, file cabinets, etc. in both offices as far as possible from the intervening wall, and especially the door, as illustrated in Fig. 8-2.

\section{PRIVACY IN OPEN-PLAN OFFICES}

In large open-plan offices with many employees, acoustical privacy is difficult to achieve between the work zones. Where privacy of a confidential nature is important in various work zones, speech originating in any one zone must be made either unintelligible or non-distracting when heard in adjacent zones. In order to achieve satisfactory privacy, a highly sound absorbent environment throughout the entire office area is mandatory. This can best be done by installing an acoustical ceiling with a very high sound absorbent rating, erecting acoustical barriers around work

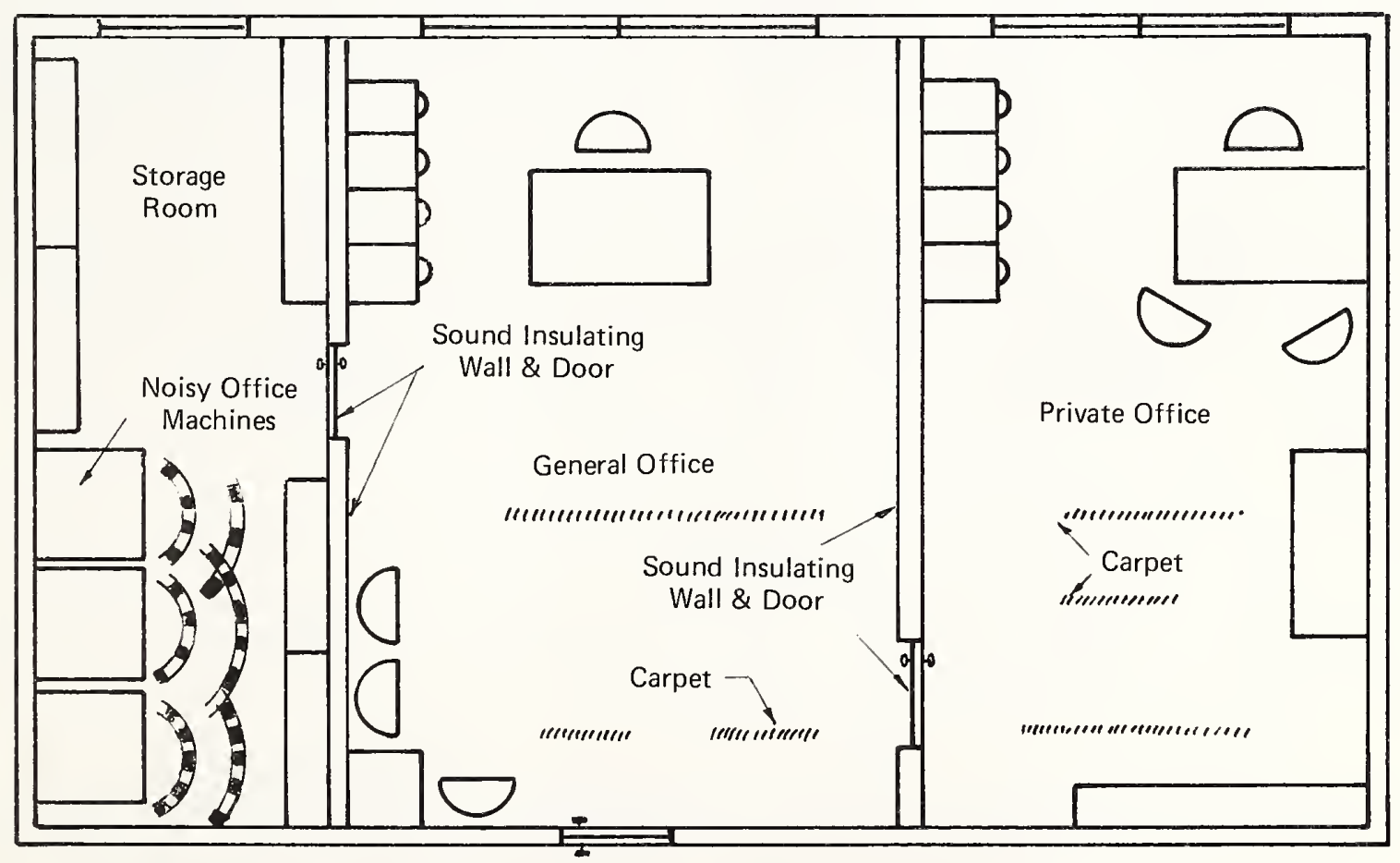

Fig. 8-2. A WELL-PLANNED OFFICE COMPLEX WHICH AFFORDS PRIVACY AND A QUIET ENVIRONMENT 
zones, introducing an unobtrusive broadband masking noise, (similar to the sound of a splashing fountain or air diffuser noise) and furnishing the office with carpeting, draperies and padded furniture. The work zones should be situated no less than 15 feet $(4.6 \mathrm{~m})$ apart. The factors controlling privacy in open plan offices are illustrated in Fig. 8-3.

The acoustical ceiling should be installed at a height of 9 feet or less. For best results the ceiling should be suspended to provide a plenum of at least 30 inches $(76 \mathrm{~cm})$. The ceiling tile or panels should have a NRC, Noise Reduction Coefficient, of between 0.70 and 0.80 . The NRC ratings usually appear on the labels of the cartons containing the tile.

The acoustical barriers should be at least 5 feet $(1.5 \mathrm{~m})$ in height, 8 feet $(2.5$ m) in length with a sound absorbing layer 2 to 4 inches (5 to $10 \mathrm{~cm}$ ) thick. Acoustical barriers encased in metal frames and faced with fabric are commercially available. Such barriers should be placed directly on the floor and positioned so that they will shield the employee from any line-of-sight sources of noise or reflecting surfaces. In other words, the barriers should be arranged so that they break up all direct paths of sound travel from one work area to another. The larger and taller the barrier the greater is its effectiveness.
Sound systems that produce specially designed masking noise are commercially available. Such systems include a number of loudspeakers which generally are installed above the suspended acoustical ceiling. The level of masking noise should be high enough to over-ride distracting noise sources in the office, but yet must be low enough not to interfere with conversation in a given work area. This points out one of the limitations or risks of using masking noise, even if it specially shaped to improve speech privacy. If the level of masking is too high, people will tend to talk louder, in order to maintain intelligibility. Consequently, their conversation will be more easily understood in other areas. Thus, instead of improvement there is actually a reduction in the degree of privacy provided by the masking.

NOISE CONTROL AT SCHOOL

$\begin{array}{ccc}\text { Many } & \text { of the criteria used for the } \\ \text { design } & \text { of acoustically desirable }\end{array}$ environments in office buildings apply also to the design of school buildings, but they often are neglected. This is rather unfortunate, because the need for a quiet environment is far more demanding in a classroom than in an office. In order to function efficiently, the teaching-learning process requires an environment that will enhance the student's ability to concentrate and study and will facilitate communication

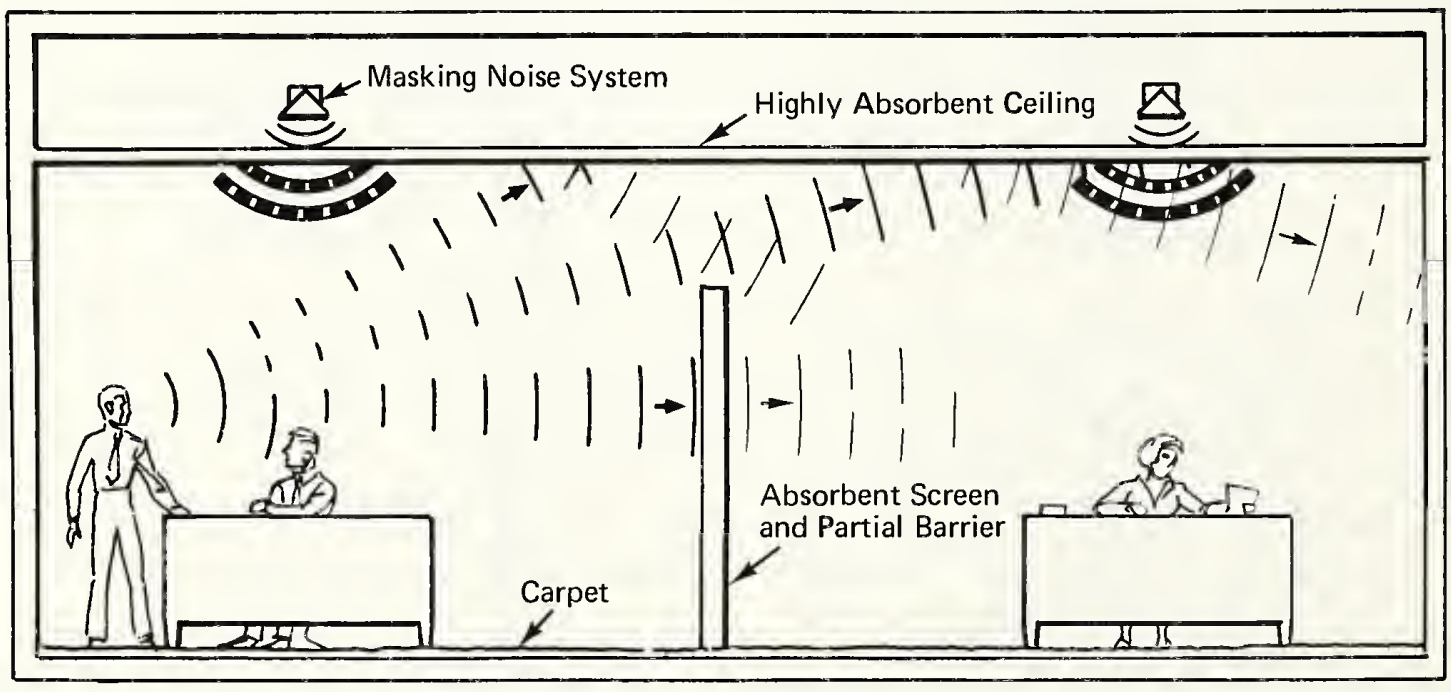

Fig. 8-3. FACTORS CONTROLLING SPEECH PRIVACY IN OPEN-PLAN OFFICES: ACOUSTICAL CEILING, SCREEN AND CARPET MINIMIZE SOUND REFLECTION. PARTIAL BARRIER ATTENUATES AND DEFLECTS DIRECT SOUND. MASKING NOISE RENDERS INTRUDING SPEECH UNINTELLIGIBLE. 
between the teacher and the students. Meeting such requirements implies creating quiet and pleasant surroundings.

\section{CAUSES OF NOISE}

Most schools tend to be too noisy. Some problems are virtually insoluble, such as those caused by the intrusion of high-intensity noise from traffic and aircraft flyovers, but most noise interference problems in classrooms stem from the intrusion of sounds from adjacent classroom or corridor activities. Since the wall, ceiling and floor surfaces and the furnishings of most classrooms are made of hard, durable materials that are good sound reflectors, the rooms generally are overly reverberant. As a consequence, intruding noises as well as those generated within the rooms build up to higher levels by repeated reflections, causing an even greater amount of distraction and aggravation. These conditions are especially prevalent in high-ceiling areas such as gymnasiums, swimming pools, cafeterias, auditoriums and large multipurpose rooms. In such areas sounds may echo or reverberate for several seconds.

The intruding noises generally are caused by student chatter or laughter in corridors, discussions in other classrooms, footsteps, movement of furniture, and on some occasions, boisterous or outright rowdy behavior of some students. However, in other cases, the transmission of noise from kitchen, cafeteria, or band room areas, or a noisy ventilation system may be the major cause of disturbance.

\section{REDUCING CLASSROOM NOISE}

If the noise seems to be coming from the corridor, even when the classroom door is closed, an expedient and often successful remedy is to install a perimeter gasket of soft resilient rubber around the door stops and at the threshold to seal the door. If the door happens to be of hollow-core construction, it should be replaced with a solid-core or soundproof door.

If on the other hand you hear conversation coming from the adjacent classroom, and there is no appreciable change in the level of disturbance whether the doors to both your room and the adjacent room are closed or open, there is a good chance that the partition wall separating the two rooms is either incapable of providing adequate sound insulation or has sound leaks. Solutions to this particular problem, as discussed in Chapter 4, require that either the noise leaks must be sealed or the wall must be modified to improve its sound insulation.

Schools or classrooms that are excessively reverberant can be treated with various types of sound absorbent materials to achieve a relatively quiet environment. Although acoustical tile applied to the ceiling of a classroom is the treatment most commonly used for this purpose, other excellent sound absorbing materials such as drapery, carpeting, and acoustic panels can be used much more effectively on the walls and floor of the room. It has been demonstrated that sound absorbent materials when placed on the floor of a large classroom or lecture hall can produce a much more desirable acoustical environment than when placed on the ceiling, even though at both locations the material would reduce the reverberation time about the same amount. The reasons for this are several. A teacher's speech is more easily heard and understood by a larger number of students when it is reflected off a hard ceiling surface than a soft sound absorbent ceiling because it follows a more direct path to the students' ears. In a classroom, the undersides of the desks and chairs and the clothing worn by the students tend to deflect the sound waves reflected from a hard floor and reduce their intensity before they reach the ears of the students.

As a rule of thumb, the acoustical environment in a classroom should approximate living room conditions. The echo or reverberation time should be about $3 / 4$ second for a moderate size room seating about 40 students. If the furniture and the surfaces of the room are made of hard, sound reflecting materials, the reverberation time can be reduced to acceptable limits by installing carpet on the floor and drapery around the windows and perhaps in the corners of the room, and if needed, a few acoustical panels encased in metal frames that can be hung on the walls. In most cases, a reasonably acceptable environment can be achieved if the total area of sound absorbent material is about equal to the floor area.

The ideal acoustical environment can best be achieved in moderate size classrooms that are fully enclosed by walls and doors which provide visual privacy and adequate sound insulation against the intrusion of both indoor and outdoor noise. The entire ceiling of the classroom and the walls 
behind and to the side of the teacher's desk should be made of hard sound reflective materials for the purpose of facilitating the projection of her voice toward the class. The sound absorbent material required to achieve the optimum reverberation time should preferably be in the form of draw drapes mounted on the windows, carpeting laid on the floor, and if needed, a few acoustical panels hung on the side and rear walls of the room.

Care should be taken to avoid making the reverberation time too short because this would produce a classroom environment that is acoustically dead. In order to teach in such surroundings, the teacher would have to raise her voice. Excessive vocal exertion for prolonged periods, even on a daily basis, leads to physical exhaustion. This is a common complaint among teachers in schools with open-plan classrooms which have no walls but depend upon highly sound absorptive surfaces and furnishings to provide adequate privacy.

of the many types of acoustical materials in the marketplace, perhaps the most effective and versatile material to use in creating an acoustically desirable environment is carpeting. A thick carpet, preferably laid on a pad of foam rubber or hair felt, will provide an amount of sound absorption comparable to that given by a typical acoustical ceiling for approximately the same area of coverage. No other acoustical material is as effective in reducing the transmission of impact sounds such as footsteps through floors to adjacent rooms or rooms directly below. And further, no other acoustical material can serve as effectively as a quiet floor covering as carpeting. Noises from footsteps, shuffling of feet, movement of furniture and falling objects, which can be extremely annoying in rooms with hard surface floors, become virtually inaudible in carpeted rooms.
Recent studies have shown that carpeting in schools exerts another positive influence that extends beyond its immediate acoustical or physical effects. Faculty members have observed a dramatic improvement in the behavior of students attending carpeted schools. Students unconsciously tend to exhibit their best living-room manners in such schools. For example, boys wearing caps remove them almost immediately upon entering the carpeted area. Noisy behavior and boisterous activity virtually vanish in carpeted areas only to be replaced with quiet conversation or subdued laughter.

\section{NOISE CONTROL IN MULTIPURPOSE ROOMS}

One of the noisiest areas in a school is the multipurpose room that serves as a dance hall, banquet room, auditorium, and at times as a kindergarten. Such rooms generally are located in the basement of the school and feature tile floors, painted cinderblock walls and gypsum board ceilings. While such room surfaces are used primarily to withstand hard use and abuse, they unfortunately cause a room to become excessively reverberant and noisy.

The most effective and adjustable acoustical treatment that can be used to control the acoustics in a inultipurpose room is a combination of draw drapery installed on the walls and around the windows, and small shag carpets laid on the floor. Thus for functions such as lectures or plays which require a short reverberation time for good audition, the drapery would be extended for maximum coverage and the carpets kept in place. However, for musical programs or dances for which a reverberation time of about 1-1/2 seconds might be desirable, the drapery would be drawn back for minimum coverage and perhaps some of the carpets would be removed. 


\section{NOISE CONTROL WHILE TRAVELING}

Prolonged exposure to noise and vibration, even at moderate levels, produces a certain amount of fatigue and irritation. This explains why most of us have experienced a feeling of exhaustion and irritability following a day-long automobile or bus trip or a cross-country jet plane flight, even though supposedly we were resting comfortably in our seats or simply relaxing while reading, conversing or watching the scenery roll by.

According to a recent survey many cars and trucks traveling at expressway speeds generate such intense infrasonic sound (i.e., sound below the frequency ordinarily considered to affect human hearing) and such high vibration levels within the vehicle that drivers are distressed by them. They experience such symptoms as fatigue, irritability, recklessness, euphoria, dizziness and lowered efficiency and vigilance.

Noise levels of about $100 \mathrm{~dB}$ in the infrasonic or very low frequency range have been measured in ordinary vehicles, with windows closed. When windows were opened the noise levels increased to $110-120 \mathrm{~dB}$. Infrasonic sounds, although they cannot be heard, may be felt or sensed as separate pulsations.

An individual person has no control of the noise levels while he is traveling in public transportation vehicles such as buses, trains, or airplanes. He has at his disposal several techniques, however, which can be used to make a substantial reduction in noise level in the automobile he drives.

\section{AUTOMOBILES}

1. Select A "Quiet" Automobile: The first course of action is to purchase or rent cars which are specifically designed to be quiet. As a rule they will prove considerably less noisy than other cars.

2. Drive at Slower Speeds: Driving at slower speeds generates less wind noise, engine noise, transmission noise, road or tire noise and reduces the low frequency noise and vibrations in the infrasonic range.
3. Close Windows for High Speed Driving: Driving with the car windows closed prevents the generation of some infrasonic noise, and reduces the intrusion of the rush-hour traffic roar. The general interior noise level, including the infrasonic noise, may be reduced as much as 10 to $20 \mathrm{~dB}$. Unfortunately, siren noise is equally diminished, therefore the driver has to be especially alert for approaching emergency vehicles. Turning off the radio and cooling fan would help to lower the interior noise level and thus make emergency signals more audible.

If air ventilation is required in the car, as on a hot summer day, the driver should use the car's air ventilation system rather than open windows. In the event a car window must be opened to provide ventilation, opening the rear window - preferably farthest from the driver - usually exposes him to the least noise. The worst condition, and unfortunately the one most commonly chosen by the driver, is to open the window nearest him. Especially at expressway speeds, this places his ear virtually within the source of very intense wind noise and air turbulence. The common driving habit of resting an elbow on the window ledge and sticking it out into the wind stream usually intensifies the noise and vibration levels. It is no wonder that a driver completing a sustained, high-speed trip under these conditions might feel exhausted, dizzy and short-tempered.

4. Use Slow Fan Speed: With rare exception, air conditioning, ventilation and defroster fans in most automobiles are excessively noisy at their high speed settings. Since fan noise is acutely dependent upon the speed of the blades, even small reductions in fan speed often result in noticeable reductions in noise output. It follows that to minimize noise, you should try to achieve adequate ventilation for the lowest possible fan speed.

5. Turn off the Radio: It is seldom recognized that sustained exposure to 
moderately loud radio sound causes some acoustic fatigue. To demonstrate this fact to those who ordinarily drive with their radios blaring, may we suggest that they drive home from work some evening with the radio turned off. They might discover to their surprise that they arrive home less tired.

6. Select Quiet Tires: Some types of passenger car tires are noisier than others. Some make a singing or whining noise, while others - like snow tires produce a low-pitched rumbling or humming noise. Generally speaking, the noise output of a tire is influenced by tread design. The buyer has a choice of three basic types of tread designs: continuous rib; block; and cross-bar, as shown in Fig. 9-1. Tires using the continuous rib pattern are relatively quiet. Furthermore, the buyer should select a continuous rib tire with a randomized or nonsymmetrical design; that is, one in which the design of each individual $\mathrm{rib}$ is different and the number of transverse grooves or slots between each pair of ribs is different. Such tread designs tend to smooth out pure-tone hum into a less objectionable broadband noise. The noisiest tires are those utilizing cross-bar designs generally found on snow tires.

7. Balance Car Wheels: Automobile wheels or tires even slightly out of balance can generate a substantial amount of vibration and low-pitched noise - often with annoying pure tone humming. Good wheel balance generally results in a smoother, quieter and less tiring ride. It also reduces tire wear.

8. Reduce Windshield Wiper Noise: Driving an automobile in rainy weather can be rather tiresome, but if one is forced to listen to the incessant noise of a chattering and groaning windshield wiper the trip can be nerve wracking. Usually, a noticeable increase in wiper noise is a sign that the rubber in the wiper blades has aged and has become stiffer and harder. An expedient though temporary measure to control such noise is to file the edges of the wiper blades with an emery board of the type found in most ladies' handbags. This will remove the hard rubber edges and expose the soft resilient rubber underneath; not only will this reduce noise but it will improve wiper action as well. The long term solution, of course, is to replace worn or dried-out wiper blades as soon as they become noisy or streaky.

If it is a question of mechanical noise, lubricating the wiper motor and linkage mechanism usually will result in somewhat quieter operation. If noisy operation persists, look for loose or worn parts, or for rubber motor mounts or gaskets
Quiet

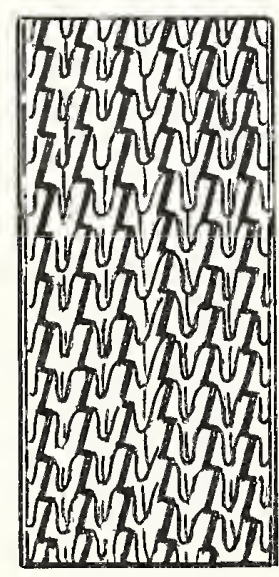

Continuous Rib
Noisy

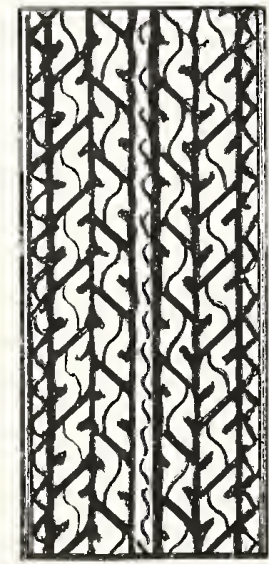

Discrete Block
Noisiest

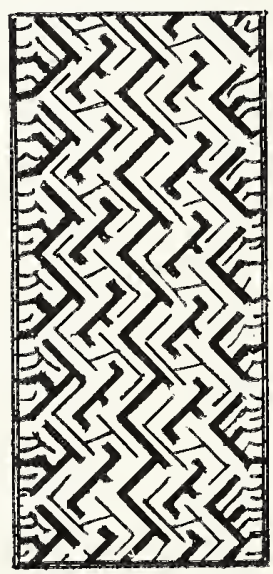

Lug or Cross-Bar

Fig. 9-1. BASIC TIRE TREAD DESIGNS IN ORDER OF RELATIVE NOISINESS 


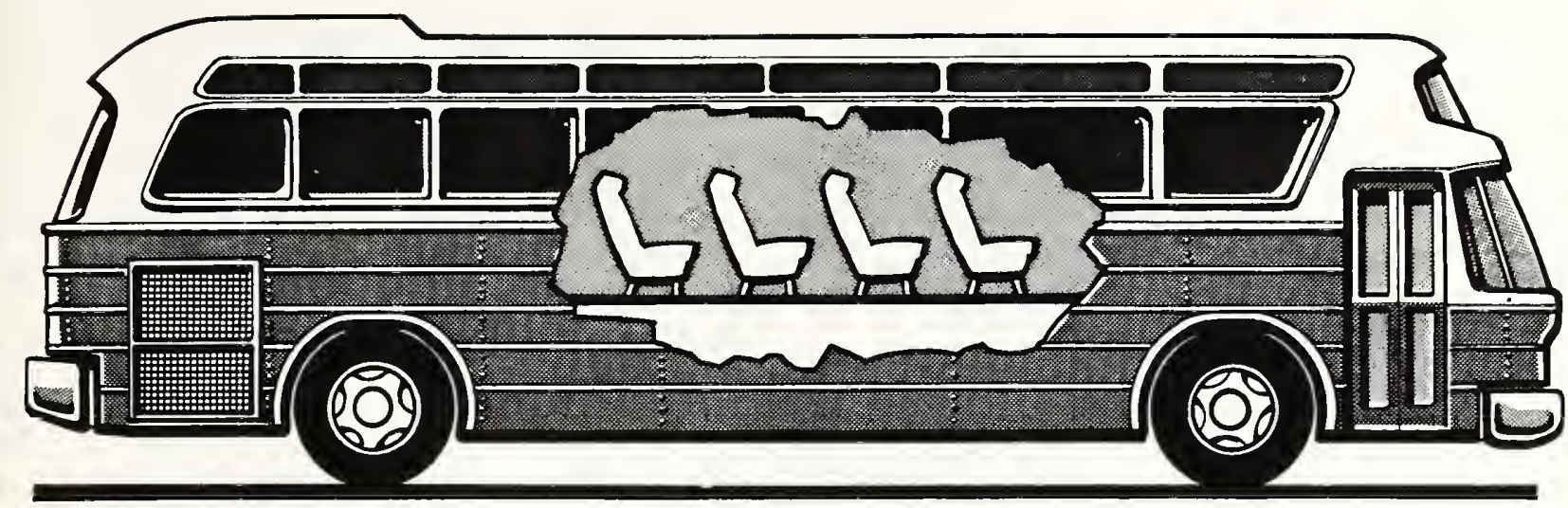

Fig. 9-2. SEATS LOCATED IN CENTER OF BUSES PROVIDE SMOOTHEST AND QUIETEST RIDE

that have become brittle and hard. Replacement of the defective parts often will restore quiet operation.

\section{BUSES AND TRAINS}

Passengers generally will find that seats in the center of the vehicle, as illustrated in Fig. 9-2, provide the smoothest and quietest ride. In buses, seats located above the wheels and near the rear engine are the noisiest and usually have the greatest vibratory motion.

\section{AIRPLANES}

Generally speaking, seats farthest removed from the windows - for example, aisle seats and those located near the center of the passenger cabin, as shown in Fig. 9-3, are the quietest and provide the smoothest ride. Window seats, as a rule, are noticeably noisier because of the windstream noise and the closer proximity to the noisy engines. Seats located in the rear or tail section of a jet plane are usually the noisiest, regardless of whether the plane has wing-mounted or tail-mounted engines.

\section{SELECTING A "QUIET" MOTEL OR HOTEL}

Having once experienced the exasperation and frustration of spending a sleepless night in some noisy motel or hotel, the weary traveler hopes that he will have better luck in picking "quiet" lodgings in future travels. He can reduce the element of chance substantially in selecting a "quiet" motel by observing a few cardinal rules, as illustrated in Fig. $9-4$ and listed below.

(a) Remember that locations near and especially within sight of traffic expressways, railroads and airports can be extremely noisy. As a precaution to avoid annoyance or interference to

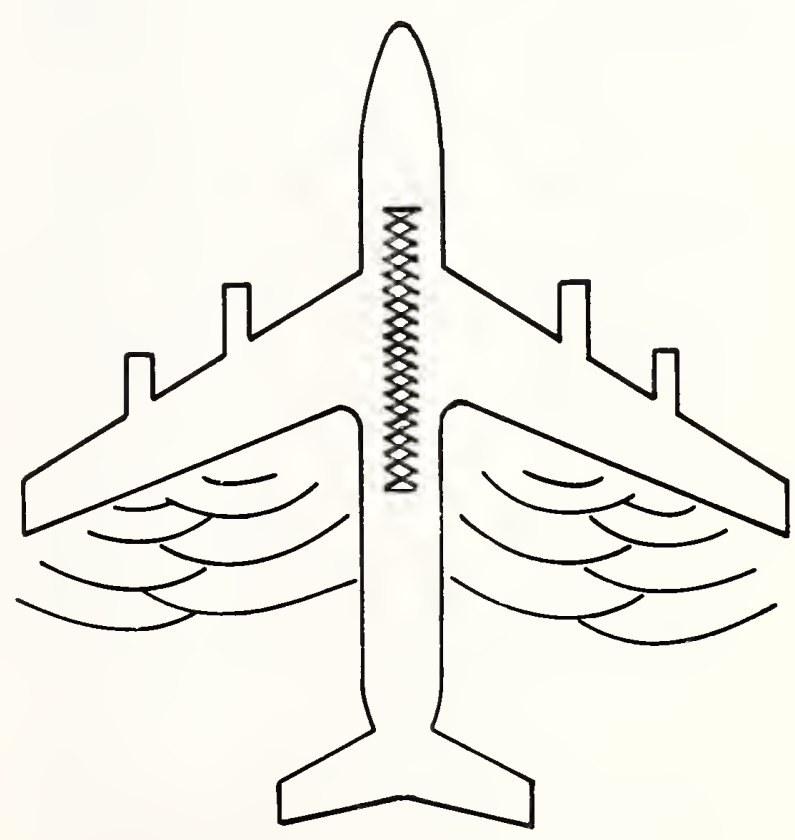

Fig. 9-3. SEATS LOCATED IN SHADED PORTION OF JET PLANE PASSENGER COMPARTMENT PROVIDE QUIETEST AND SMOOTHEST RIDE 


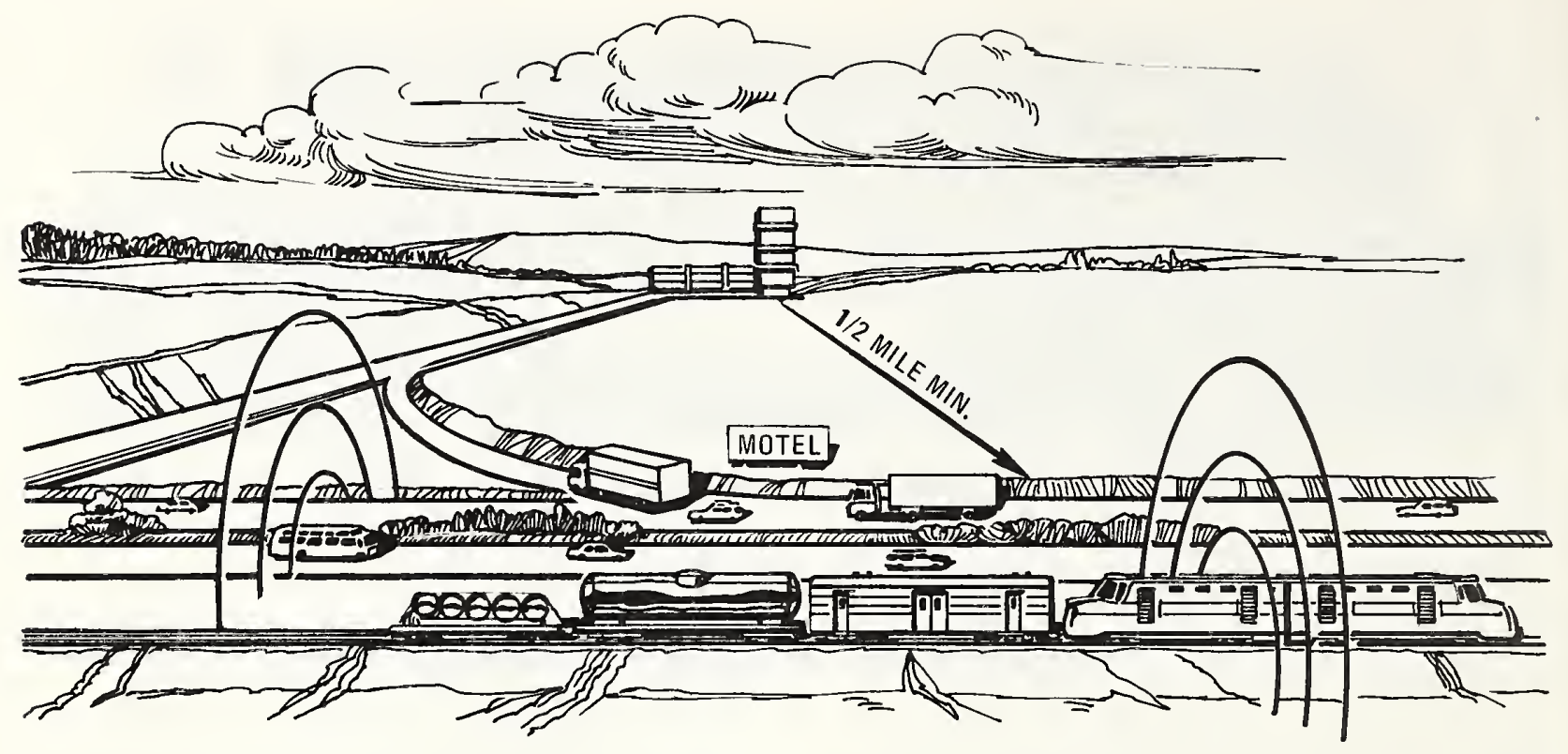

Fig. 9-4. TO MINIMIZE DISTURBANCE FROM TRANSPORTATION NOISE, GIVE PREFERENCE TO MOTELS AT LEAST 1/2 MILE FROM EXPRESSWAYS AND RAILWAYS

sleep from traffic and rail noise, try to select motels or hotels that are at least $1 / 2$ mile $(800 \mathrm{~m})$ away from such sources. If you are traveling by car, you can use the odometer or mileage gauge to measure the distance.

However, hotels and motels can be designed and constructed to prevent the intrusion of traffic noise, even at road-side locations. Such sound-insulating construction would consist of brick or concrete block exterior walls, non-operable double-pane windows, solid-core doors with perimeter gaskets, and central air-conditioning systems. The traveler who desires both the convenience of a road-side motel and quiet accommodations should select a motel that features such construction. In addition, he should choose a room on the quiet side of the notel which faces away from the road.

(b) Be especially careful to select hotels or motels that are not located in the path of direct, low-altitude aircraft flyovers. Unfortunately, finding a motel which is free of annoyance from nighttime jet flights may be difficult because of the intensity of the noise and the great distances that are required to attenuate it to acceptable levels simply by air absorption alone. Ordinarily, the traveler faced with the problem of determining whether or not jet aircraft flying in the vicinity of a given motel might disturb his rest can resolve it in one of three ways.

(i) If he is a trusting soul, he can ask the registration clerk whether or not aircraft noise is a source of disturbance at night.

(ii) He can conduct a simple "talk test" outside the motel. If it is possible to converse normally with a person three to five feet away without difficulty or disruption during a typical aircraft fly-by, the need not worry.

(iii) He should request a trial demonstration inside a given room to determine for himself whether or not aircraft noise is audible and possibly annoying.

(c) Check carefully before selecting motels or hotels with window or wall-mounted air conditioners. Window or wall-mounted air conditioners generally are relatively noisy devices, especially models that are five or more 
years old. The noise levels produced by such units may disturb one's sleep. Furthermore, the air intake and exhaust ports of such units act as speaking tubes or noise leaks, thereby allowing the transmission of exterior noise into the room. As a consequence, even if you choose not to operate the unit because of the noise, you may well be disturbed by the noise radiating from air conditioners in adjoining rooms or other areas, not to mention noise associated with the late arrival or early departures of motel guests.

(d) Noise can be expected to intrude into motels or hotels with louvered windows or doors. Although open louvers are often desired for fresh air ventilation, they unfortunately constitute an open path for the intrusion of outdoor noise. Because they are inherently difficult to seal, closed louvers provide very little protection from outdoor noise.

(e) Motels built with wood-frame construction require exceptional care in order to be made well-isolated from noise. Party walls in wood-frame structures often are lightly made, and sounds from occupants in adjacent rooms pass readily through. Unless dwelling units are made up as separate small cabins, privacy may be limited. Sometimes owners install sound absorbent acoustical tiles in an attempt to defeat the problem, but this doesn't inhibit the passage of sound between dwelling places.

The traveler should not be misled by the presence of acoustic tile on the ceiling or walls of a particular motel. This is no guarantee that the room is "soundproofed". While such treatment effectively reduces the buildup of noise within the room, it is totally ineffectual in preventing the intrusion of noise from outdoor sources or from adjoining rooms or other areas within the building.

Generally speaking, most motel or hotel rooms do not require any acoustical treatment on ceilings or walls; the ordinary room furnishings of carpeting, drapery, bedspreads and mattresses and padded furniture are good sound absorbers and prevent the room from becoming too reverberant and noisy. Have you every wondered why your bedroom is so much more quiet than, say, your kitchen or bathroom? The sound-absorbing furnishings in the bedroom make the difference. It also explains why the "Bathroom Baritone" rarely sings in the bedroom - there his voice would sound weak and flat.

(f) Select motels of brick, concrete or masonry construction. Such motels generally have reasonably massive party walls and floors. In addition look for thick carpeting and drapery, quiet air conditioning and ventilation systems, well-fitted solid-core doors, and sealed non-operable plate glass windows. These features combined tend to provide a quiet, restful interior environment free from the intrusion of outdoor noise, or noise from adjoining rooms or other areas within the building.

\section{SELECTING A "QUIET" ROOM}

Select a room on the "Quiet" side of the motel (See Fig. 9-5). Since buildings are relatively large and massive structures, they can effectively deflect sound or noise. The larger the building, the greater its effectiveness as a noise barrier. When a sound wave strikes a building, some of the sound energy penetrates into the building and is absorbed; the remaining energy is reflected or deflected by the building in other directions. That side of the building receiving the full force of the sound wave will, of course, be relatively noisier than the opposite or shielded side.

Therefore, when selecting a motel, the traveler should remember that rooms on the side of a motel that faces a major source of noise, such as an expressway or railroad, may be objectionably noisy; whereas, rooms on the opposite side in the acoustic shadow of the building may be relatively quiet.

In addition, rooms on the "quiet" side of the motel ordinarily are disturbed less frequently by noise generated on the motel premises, such as noises caused by swimming pool activities, patio parties, or the traffic associated with guest registration.

Other tips on selecting a "Quiet" motel or hotel room:

(a) Avoid rooms which are adjacent to or near stairways, elevators, or areas containing vending machines, cooling towers, fans, or air conditioning equipment. The noise radiation from such equipment or from people using the vending machines may be quite disturbing. See Fig. 9-6. 


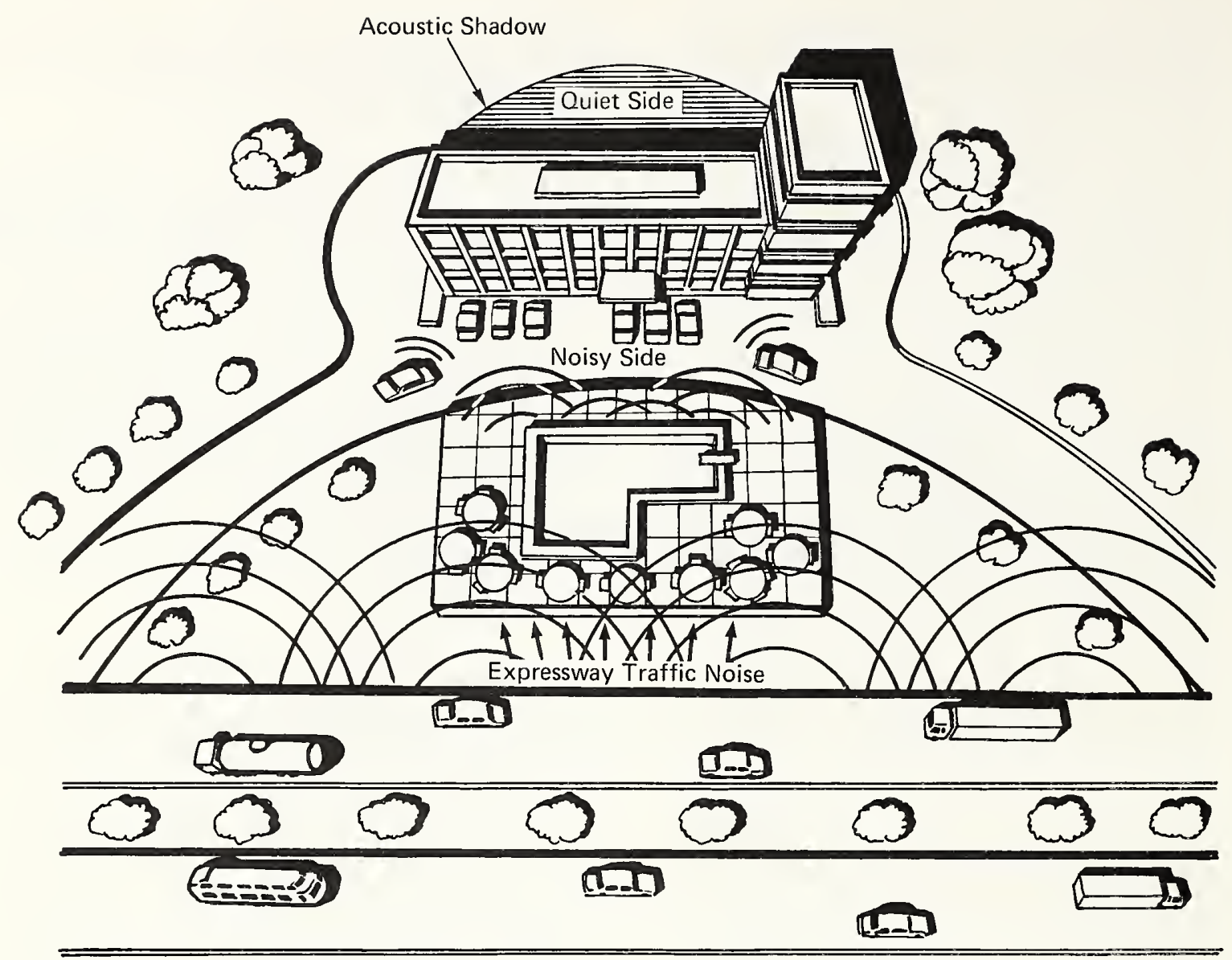

Fig. 9-5. TO MINIMIZE DISTURBANCE FROM TRAFFIC NOISE SELECT ROOMS ON "OUIET" SIDE OF THE MOTEL

(b) Avoid rooms located on the ground floor level, if noises from footsteps, parking lots or plumbing are particularly annoying to you. Rooms located on the top floor provide the most isolation from such disturbances. (c) In large hotels in downtown locations, rooms facing an inner courtyard are usually much less noisy than rooms overlooking the street. The courtyard, however, can act as a speaking tube for noises generated within it. 


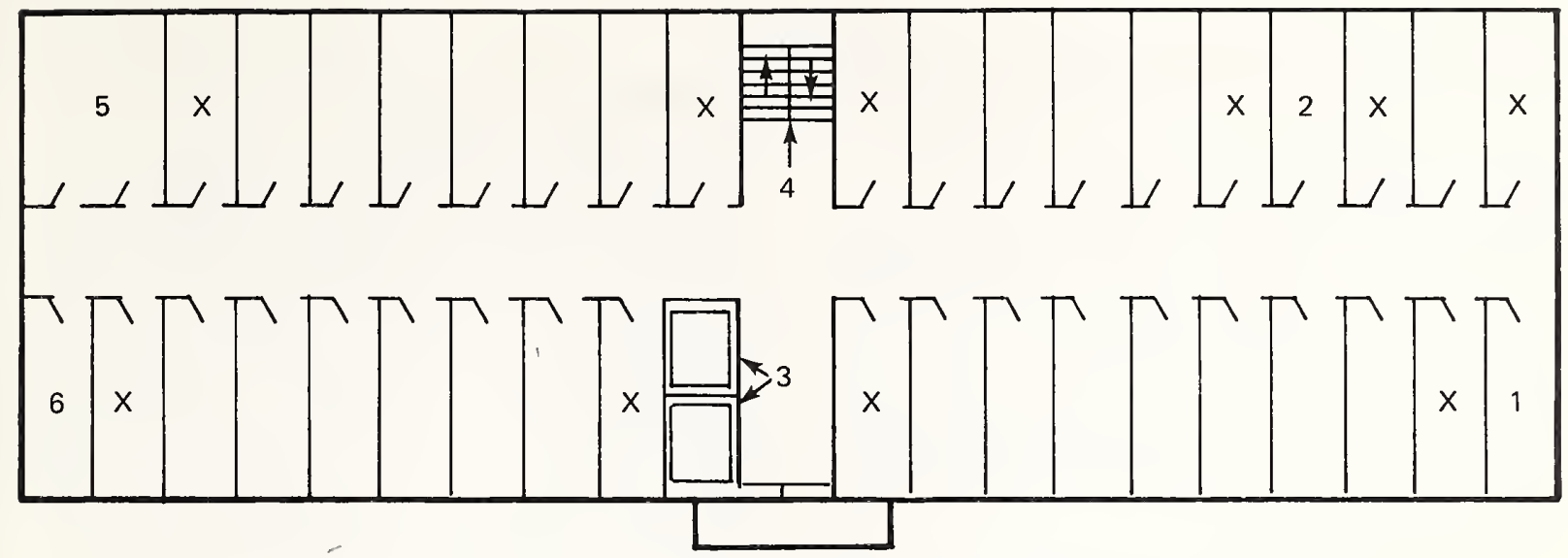

X Marks the Location of Potentially Noisy Guest Rooms

1. Mechanical equipment room

2. Vending \& ice machine room

3. Elevators
4. Staircase

5. Conference room

6. Telephone room

Fig. 9-6. TO AVOID DISTURBANCE FROM NOISE, SELECT HOTEL OR MOTEL ROOMS THAT ARE AWAY FROM NOISY AREAS OR EQUIPMENT ROOMS. 


\section{INTRODUCIION}

As an individual you can apply the principles of noise control given in the previous chapters. By doing so, you can protect yourself from the harmful and irritating effects of noise from many sources. Yet, there are some sources of noise which affect you, and over which you have no direct control. What can you do in such cases?

You can exhaust simple, informal remedies: Talk to your neighbor; call the construction foreman down the street; call the relevant officers in your town, the councilman, public health officer, the licensing bureau, or the official who issues building permits. People say, "You can't fight City Hall", but in fact you can.

As problems of noise intrusion have developed, groups of citizens have already made their voices heard. You will find that there are agencies established at severa1 levels of government -- and you can get your government to help you, to mediate between you and the parties responsible for the noise problem. The following is a list of some existing agencies dealing with noise abatement.

\section{FEDERAL NOISE REGULATION}

The Noise Contro1 Act of 1972 represents the first major Federal legislation in the field of noise control. The Act requires the U. S. Environmental Protection Agency to develop and publish information concerning noise levels that may be hazardous, and to set standards defining permissible noise levels for products that have been identified as major noise sources. These may include such products as power lawn mowers, household appliances, and room air conditioners.

Although EPA has primary responsibility for most Federal efforts to control noise, other agencies are also concerned with special areas of noise control.

Department of Transportation:

The Federal Aviation Administration (FAA) sets criteria and standards for controlling aircraft noise. EPA advises the FAA in fulfilling its responsibilities, while much of the research and experimental testing for aircraft noise abatement is performed for FAA by the National Aeronautics and Space Administration and by the U. S. Air Force. The Federal Highway Administration, also within the Department of Transportation, has adopted Noise Control Standards and Procedures for use in planning and design of highways to assure that measures are taken to hold highway noise to levels that are compatible with various land uses.

Department of Labor: The Occupational Safety and Health Administration sets and enforces regulations, under the Occupational Safety and Health Act of 1970, that are designed to protect the hearing of workers. Authority is limited to regulation of companies engaged in interstate commerce. Under this Act, the Department of Health, Education and Welfare performs research to define occupational noise limits needed to safeguard the hearing of workers. The Labor Department relies upon results of this research in setting noise standards.

Department of Health, Education and Welfare; Department of the Interior: Under the authority of the Federal Coal Mine Health and Safety Act of 1969, the National Institute for Occupational Safety and Health, Department of Health, Education and Welfare is responsible for the development of noise standards to protect the hearing of miners. The enforcement of these standards is the responsibility of the Mining Enforcement and Safety Administration, Department of the Interior.

The Department of Housing and Urban Development: The Department of Housing and Urban Development (HUD) has developed criteria for the sound insulation characteristics of walls and floors in row houses, nursing homes, and multi-family housing units. These criteria must be met by housing of this type in order to qualify for HUD mortgage insurance.

Many other Federal agencies, such as the National Bureau of Standards and the National Science Foundation, are concerned with research in noise control and abatement in factories, homes, offices, and commercial work areas. 
3. Authority would exist for the

For almost a century and a half the control of noise by regulation within the United States has been a matter of concern for city governments. It is only within the last decade that some states have seriously entered the field of noise regulation. Over 400 municipalities (representing a combined population in excess of 60 million people 30 percent of the U.S. population) have enacted noise regulations; however, until recently, there has been very little leadership at the state level except for the enactment of regulations requiring mufflers on motor vehicles. An up-to-date compilation of various city and state noise control ordnances is maintained by EPA (Washington, D. C. 20460)

Both local and state noise control laws are usually classed as general nuisance laws which include a prohibition of "unnecessary," "excessive," or "unreasonable" noise. These criteria are often too subjective to be effectively enforced. When vigorously challenged, this type of noise control law tends to be struck down by the courts because it is too broadly worded for objective enforcement. What is "unnecessary" or "unreasonable" for one person may be "acceptable" to another.

Noise control laws which are based upon performance standards appear to be far superior to nuisance laws if they are properly written and correctly applied. Performance standards typically specify the maximum allowable noise level at a given point; and this requires monitoring equipment and trained personnel for enforcement.

A promising trend has been the adoption of noise control laws with performance standards (such as specified in the model ordinance prepared by the National Institute of Municipal Law Officers, Washington, D. C. 20006) and the establishment of offices of noise abatement to supervise enforcement. The advantages of such legislation and of establishing a regulatory agency are severa1:

1. An Office of Noise Abatement can act as a focal point for activities in noise control regulation, enforcement, and public education.

2. A staff can be assembled that is specifically trained to respond to complaints and monitor noise levels. office of Noise Abatement to use initiative in seeking out the worst offenders.

4. An office of Noise Abatement would have legal authority to advise zoning commissions on the noise impact of different zoning plans under consideration.

The main disadvantage is the cost of such a program. Although this cost is not high relative to other kinds of pollution controls, it represents yet another demand on city, county and state budgets.

\section{CORRECTIVE ACTION PROGRAMS}

To be effective in solving community-wide noise problems the average citizen must make himself heard -- as in Chicago where an organization called Citizens Against Noise persuaded the City Council to pass an effective antinoise ordinance in 1971; and as in New York City where Robert Baron and Citizens for a Quiet City, Inc., succeeded in establishing a Bureau of Noise Abatement.

Obviously, it is first necessary to familiarize yourself with the various regulations (Federal, state, and local) which affect your "noise environment." This familiarization need not be a close and detailed reading of all the applicable laws. At the local level, a telephone call to the police department and the city or county council is a starting point for determining what laws exist, if any, relating to noise control and their applicability to your particular case. The matter of noise in a community could also be brought up at a local civic association meeting - many voices can be more effective than one!

At the state level, you may write or call your elected officials in the legislature to inquire what they are doing about an effective noise control law in the state. The governor's office should also be contacted. Again, it is a matter of being heard. If enough organizations and private citizens ask probing questions about noise pollution, elected officials will take notice of this interest and corrective action will be forthcoming.

Although the activities of many Federal agencies have some bearing on noise control, it is the U. S. Envirommental Protection Agency that takes the lead in coordinating these activities and initiating others. Write to the EPA Office of Public Affairs 
(Washington, D. C. 20460) for publications, general information, and answers to specific questions on noise control. Your voice, individually and collectively, can be heard at the Federal level through many channels of communication - by making your concern known to your representative and two senators in Congress, and to the President of the United States. You can also exert influence by urging EPA and other Federal agencies to develop and enforce noise control standards.

A corrective action program, to be effective, must involve powerful political groups in the community. Since both government and industry ultimately respond to public demands, the only real solution to the overall noise problem is a rising public awareness of the dangers of noise and a persistent demand for effective noise control.
Some noise problems may be quite local in character and are best solved on a neighbor-to-neighbor basis. For example, while nuisance laws might be invoked to engage the police in quieting a neighbor's dog, such tactics may do little to promote friendly neighborhood relations! Perhaps a better approach to solving problems of this kind might be to raise the issue of noise at a meeting of your local neighborhood association, or at a block party, attended by your neighbors. You might suggest appointment of a "noise warden" whose duty it would be to register complaints to those causing a disturbance. If such complaints were brought to the attention of the offender in a diplomatic way, and perhaps in indirect fashion through an appointed neighborhood representative, sensibilities may be spared - and more positive effect achieved! 
The following books are recommended to the reader for more detailed information on noise control:

"A Guide to Airborne, Impact, and Structureborne Noise Control in Multifamily Dwellings", HUD FT/TS-24, prepared for the U. S. Dept. of Housing and Urban Development by R. D. Berendt, G. E. Winzer, and C. B. Burroughs, National Bureau of Standards, January 1968.

"Solutions to Noise Control Problems in the Construction of Houses, Apartments, Motels and Hotels", Owens-Corning Fiberglas, December 1963.

"A Study -- Insulating Houses from Aircraft Noise", U. S. Dept. of Housing and Urban Development, HUD TS-19, May, 1967.

"Noise Assessment Guidelines", U. S. Dept. of Housing and Urban Development, TE/NA-171, August 1971 .

"Sound, Noise, and Vibration Control", Lyle F. Yerges, Van Nostrand Reinhold Company, New York, 1969.

"Acoustical Designing in Architecture", Vern 0 . Knudsen and Cyril M. Harris, John Wiley \& Sons, Inc., New York, 1950.

"Sound Control Constructions, Principles and Performance", United States Gypsum, 1972.

"Handbook of Noise Control," Cyril M. Harris, Ed., McGraw-Hil1 Book Co., Inc., New York, Toronto, London, 1957.

"The Tyranny of Noise", Robert Alex Baron, St. Martins Press, New York, 1970.

"Handbook of Noise Measurement", Arnold P. G. Peterson and Ervin E. Gross, Jr., (Seventh Ed.), General Radio Co., 1972.

"How You can Soundproof your Home", Paul Jensen and Glenn Sweitzer, Lexington Publishing Co., Lexington, Mass., 1974.

"The Noise Around Us", Including Technical Backup, Commerce Technical Advisory Board on Noise Abatement, CTAB-71-1, September, 1970.

"Regulation of Noise in Urban Areas - A Manual Prepared for Public officials, Managers and Environmental Engineers", W. S. Gatley and E. E. Frye, University of Missouri, Ro11a, 2nd edition, 1972.
"Noise from Construction Equipment and Operations, Building Equipment, and Home Appliances," NTID300.1, U. S. Environmenta1 Protection Agency, December 31, 1971.

"Effects of Noise on People," NTID300.7, U. S. Environmental Protection Agency, December 31,1971 .

"A Survey of Studies on the Effects of Noise on Performance and Health within the Socialist Countries," E. Gullian, Report to the Committee on Hearing and Bioacoustics (CHABA), sponsored by the Occupational Safety and Health Administration (OSHA), 1974.

"Effects of Noise on Physiological State," Proc. Conf. Noise as a Public Health Hazard (American Speech and Hearing Administration, G. Jansen, Pp. 89-98 (1969).

"Annoyance Reactions from Aircraft Noise Exposure," R. Rylander, S. Sorensen, and A. Kajland, J. Sound and Vibration 24, 419-444 (1972).

"Some Effects of Infrasonic Noise in Transportation," M. J. Evans and W. Tempest, J. Sound and Vibration 22, 19-24 (1972).

"Acceptability Criteria for Noise in Large Offices," E. C. Keighley, J. Sound and Vibration 11, 83-93 (1970).

"A Laboratory Study of the Nuisance Due to Traffic Noise in a Speech Environment," C. G. Rice, B. M. Sullivan, J. G. Charles and C. G. Jordan, J. Sound and Vibration 37, 87-96 (1974).

"Effects of Noise on People," J. D. Miller, J. Acoust. Soc. Am. 56, 729-764 (1974).

"Proposed Damage-Risk Criteria for Intermittent Noise Exposure", W. D. Ward, Proc. XV International Congress on Occupational Hea1th, Paper AIV-36, pp. 143-149, September 1966.

"Environmental Acoustics", L. L. Doelle, McGraw-Hil1 Book Co., New York, 1972.

"Acoustical Manual: Apartment and Home Construction," NAHB No. 315.04, NAHB Research Foundation, Inc., June, 1971. 
"Susceptability to TTS and PTS", W. Dixon Ward, Proc. of Internat. Congress on Noise as a Public Health Problem, Dubrovnik, Yugoslavia, May 13-18, 1973.

"Adaptation to Community Noise - A Case Study", E. Jonsson and S. Sorensen, Journ. Sound and Vibration (1973) 26 (4), 571-575.
"Environmental Noise Control," E. B. Magrab, John Wiley \& Sons, New York, N. Y., 1975.

"Environmental Noise \& Vibration Control," M. E. Zaret, Western New England College, 1975. 
A-weighting Network, 4-6, 102

Absorbers, 30, 36

Distribution for maximum effectiveness, 34, 131

Materials, 30, 35

Space, 35

Wa11, 35

Absorption, 29-31, 34, 49

In air, 29-31

In buildings, 30, 34, 49

In materials; 30,31

Acoustical Ceiling, 51, 55

Acoustical Fatigue, 14, 41, 133-134

Acoustical Hangover, 11

Acoustical Lining, 28, 36, 54, 55

Acoustical Materials, 28, 31

Acoustica1 Pane1s, 35, 131

Acoustical Perfume, 41-44

Acoustical Ratings, 16, 61, 71

Acoustical Sealants, 28, 32

Acoustical Shadow, 137

Acoustical Shielding, Barriers, 35, 36, 40, $59-66,130$

Acoustical Treatment, 16, 34, 36, 50

Acousticians, 2, 88

Acoustics, Rooms, 130-132

Air Absorption, 29-31, 34

Air Bleeding and Venting, 120

Air Chambers, 81

Air Compression, 1, 2, 29, 81

Air Compressors, 47, 70, 71, 112

Air Conditioners, 23, 47, 70, 110, 136 Installation, 23, 47, 136

Noise contro1 of, 47, 70, 86, 110

Types, 110,136

Air Filter, 54, 110

Air Hammers
Air Rarefaction, 1, 2, 29

Air Return Ducts, 59, 60, 70-71, 73, 98

Air Transfer Ducts, Grilles, 59, 60, 98

Airborne Sound, 2, 17, 45-47

Absorption, 29-31, 49-50

Insulation, 50, 51

Transmission, 45-49

Aircraft Noise, 3, 4, 7, 41, 87-88, 97-98, $110,127,131$

Flyover Noise, 3, 87-88, $99,127,131$

Helicopter Blade Slap, see Sonic Boom, 122

Intrusion, 35, 87-88, 127

Passenger Compartment Noise, 135

Airflow Noise, 23, 71, 77

Ducts, 21, 74-77, 113

Fans, 18-20, 70, 71

Grilles, 77, 113

Ventilation systems, $18-20$

Airport Noise, 86, 87, 97

Impact on residential areas, 86, 97

Alarm Clock, 4, 105, 110

Alarms, 110

Ambient Noise, 8, 127

Antinoise Ordinances, 141

Apartment Selection, Chapt. 6, pp. 97-101

Appliances, 9, 28, 29, 110-112, 114

Application as noise generators and detectors, 104

Design criteria, 9, 16-21, 29

Installation, 26-29, 38

Isolation, 28, 51-57

Noise control, 28, 29, 52-55

Noise rating, 16,71

Attenuation of Sound

Barriers, 59-66

Distance, 30, 33-34

Doors, 90

Wa1ls and floors, 61

Windows, 87,88

Attics, 91-92

Auditoriums, 30, 131-132

Automobile, Noise Control, 15, 133-134

Background Noise, 8, 127 
"Acoustical Perfume", 41-44

Masking, 8, 41-44, 60

Baffles, 37

Balance, Machinery, 20, 21, 72

Tires, wheels, 134

Ballast, Fluorescent Lamp, 114

Barriers, Wa11s, Fences, 35, 36, 40, 59-66, 87

Baseboard Heaters, 78-79, 116

Basements, 32, 57-58, 92-96

Bathroom Baritone, 32

Bathrooms, 32, 58, 59-66, 81

Bedrooms, 4, 32, 58, 72

Belt Noise, Drumming, 73, 113

Bending of Sound, 32

Binaural Hearing, 102

Birds, 99, 110

Blade-passage Frequency, 70

Blade Tip Speed, 19

Blenders, 51, 110-.111

Blowers, 20, 29, 70, 73, 111

Blade Passage Frequency, 70

Mounting, 70-74

Bowling Alley, 111

Broadband Noise, 7, 41

Building Sites, Orientation, 97-101

Burner Jets and Nozzle Noise, 78

Bus Noise, 99, 111, 135

Community Disturbance, 99

Passenger Disturbance, 29, 135

Cabinets, 28, 29, 53-54, 62, 63, 111

Installation, 53-54

Insulation, 54

Noise reduction, 28, 54

Cafeterias, 131

Can Opener, 51, 111

Carpet, 30, 31, 49, 55, 58, 65, 98, 130-132 Acoustical Properties, 30, 31, 49, 55
Sound Absorber in Classrooms, 130-132

Caulking, 28, 58, 61-62, 63, 65, 88, 105, 129

Ceilings, 35, 54-58, 65, 73

Central Air Return, 59, 70

Corridors, 59, 70

Doors, 59

Problems, 59, 70

Chain Saw, 13, 40, 106, 111

Children's Voices, 57-58, 101, 111

City Planners, 98

Zoning, 86,98

Classrooms, Noise Control, Acoustics, 129-132

Clocks, 111

Closets, 59

Furnace Installation, 59, 70, 73

Clothes Dryer-Washer, 17, 81, 111

Combustion Noise, 74, 78, 111

Burner Jets, Nozzles, 78

Compactor, 112

Computers, 127-128

Compressed Air Line, 112

Compression of Air, 1, 29, 81

Compressors, 47, 70, 71, 112

Concentration of Sound in Corners, 34

Cooling Towers, 101, 137

Corridor Noise, 30, 46, 58, 112, 131

Reduction, 46

Shielding, 46

Transmission, 46

Couplers, Flexible, 23, 38, 79, 81

Courtyard, 32, $101,112,138$

Craw1 Spaces, 92, 96

Creaking, 66-70, 112

Cross Talk, 75-76, 112, 129

Also see Flanking Transmission

Cylindrical Wavefronts, 33 
Damage to Hearing, 1, 8-13, 15, 102

Damper Noise, 23, 77, 87, 112

Duct Noise, 113

Decibel Scale, 3 Definition and Application, 3 $\mathrm{dB}(\mathrm{A})$ Notation, 3-5

Diffraction, 32

Diffusers, 77, 112

Directionality of Sound Source, 31

Dishwasher, 51, 81, 113

Dog Barking, 86, 99, 113

Domestic Noise

Detectors, 104

Sources, 104

Doors, 35, 57, 59, 90, 91

Air Leaks, 35

Bel1s, 12, 29

Creaking and Rattling, 112

Exterior, 86, 90-91

Flanking Paths, 47

Gaskets, 52, 57, 59

Ho 11 ow-core, 59,87

Interior, 52, 57, 59

Knocker, 113

Mounting, 52

Prehung, 52, 90, 104

Sealing Against Noise, 32, 58

Slamming, 54, 113

Solid-Core, 52, 58, 87, 91, 98

Storm door, 86,87

Sound Insulating, Soundproof, 52, 57, 58, $87,128,129$

So und Leaks, 59

Threshold Seal, 52, 59

Drain Pipes, 81, 85

Installation, 81, 85

Isolation From Noise, 81,85

Quiet Design, 81, 85

Drapery, 30-31, 49, 130-131

Acoustical Properties, 30-31, 34, 49

Multipurpose Rooms 131, 132

Drills, Pneumatic, Power, 105

Drop-closures, Doors, 59

Drumming Noise, 73, 113

Belts, 73, 113

Panels, 73

Reduction, 73, 113

Ducts, 74-77, 113

Absorbent Treatment, 28, 36, 75, 76
Ballooning, 77

Central Return, 59, 70, 76-77

Creaking, 74, 77

Criteria, 21-33, 76-77

Crosstalk, 75-76, 129

Damper Noise, 23, 77, 87, 112

Design Criteria, 21-23, 76-77

Expansion Noise, 74

Flexible Connectors, 23

Flexing, 23

Flow Noise, 77

Mounting, 77

Resilient Hangers, 77

Sealing Against Noise, 28

Sound Transmission, 74

Vibration Transmission, 74

Ear, 9-12

Damage to, $1,8-13$

Desensitization, 10

Drum, rupture, 10

Frequency Response, 6

Resolution of Sound, 3-6

Sensitivity, 3-6

Sound and Vibration Detector and Locator, 104

Ear Phones, 57

Ear Protectors, 40

Eccentricity, 21

Echoes, 31, 32

Electrical Appliances, 114

Choosing Quiet Units, 16, 17, 29

Design Criteria, 16, 17, 29

Electromagnetic Noise, 114

Electromechanical Equipment, 114

Enclosures of, 40, 127

Noise Reduction, $18-21$

Electronic Air Filter, 114

Elevators, 101, 114, 137

Noise Isolation, 47

Shaft Noise Transmission, 114

Enclosures for Equipment, 40, 127

EPA, Environmental Protection Agency, 140-142

Equal-1oudness Contours, 4-7, 14

Exhaust Noise 7, 14

Expansion and Contraction, 114

Ductwork, 76-77

Heaters, 78

Piping, 78, 80 
Extended Source, 34

Fans, $18-20$

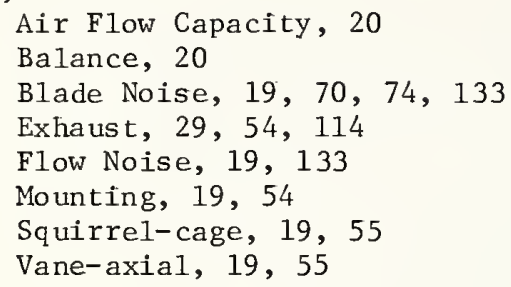

Faucets, 81,114

Dripping, 81

Flow Noise, 81

Valve Noise, 81

Water Hammer, 81

Federal Noise Regulation, 140-142

\section{Filters}

Air, 54

So und, 4-6

Flanking Noise, 47-48

Flexible Connectors, Hoses, Ducts, 23, 38, 73, 79,81

Floor-Ceiling Assemb1y, 35, 65

Floors, 65-69, 114

Foots tep Noise, 34-35, 55, 58, 59, 65

Impact Noise, 55, 58-59

So und Insulation, 65-66

Squeaking, 66-69

Flow Noise

Air, 2, 29

Water 78-81

Flow Resistance, 20-23

Flow Velocity, 20-22, 77, 80

Reduction Techniques, 20-23, 73, 77, 80

Fluorescent Lights, 114

Footstep Noise, 35, 55, 58, 59, 65, 130-132

Forced Air Heating Systems, 59

Frequency, 3, 6, 29

Frictional Resistance, 20-23, 31

Furnace, 71, 78-79

Installation, 71, 78-79, 86

Noise, 71, 77-78, 79

Garage Noise, 101
Garbage, Trash, 29

Cans, 29, 41, 115

Collection, 115

Compactors, 112

Disposal Units, 4, 53, 54, 115,

Garden Tools, 115

Gaskets, 28, 52, 58-59, 87

Central Return, 70

Design Criteria, 77, 115

Noise Output, 77, 115

Test for Noisy Grille, 77

Grinders, 116

Gunshot, 10, 12-13

Gymnasiums, 131

Hairdryers, 105

Hallways, Corridors, 30, 46, 58, 112

Headsets, Earphones, 12, 57

Hearing Aids, 11

Hearing Mechanism, 4

Hearing Damage, by Noise, 1, 8-10, 15, 102 Hair Cel1 Damage, Nerve Damage, 11 NIPTS, 8-9

Permanent Threshold Shift, 8-9, 11

Ruptured Ear Drum, 10

Sensorineura1 Hearing Loss, 8, 11-13

Hearing Precautions, 8, 12

Ringing in ears, 11, 12

Temporory Threshold Shift, 8-9

Tickle Sensation, 11

Tinnitus, 11

Hearing Protection, 12-14, 40-41

Ear Plugs, Helmets, 12-14

Stapedius Reflex, 10

Heating Systems, 70, 78-79, 116, 117, 120, 122

Baseboard, 78-79, 116

Electrical 78,-79, 116

Forced Air, 78-79, 116

Furnace, 78-79, 86, 116

Hot Water, 78-79, 116, 120

Steam Heat, 78-79, 116, 120, 122

Helicopter, Blade Slap, see Shock Wave, 122

Hertz, 3, 6

Hi-Fi Systems, 13 
Homes

Noise Control, Chapter 4, PP 45-85, $87,97-98$

Hotels, 135-137

HUD, Dept. of Housing and Urban Development, 140

Hum, 74, 79, 117

Humidifiers, 17,117

Hydraulic Controls, 117

Ice Crusher, 51, 117

Imbalance Noise, Rotating Parts, 20

Impact Noise, 17, 49, 58, 117, 130-132

Cabinets, 54

Footsteps, 55, 58, 65, 130-132

Reduction of Impulsive Forces, 17-18, 49

Impactor, 117

Incinerators, 117

Indoor Noise, 45

Industrial Noise, 3, 14, 15, 41, 97, 102

Infrasonic Sound, 133

Distress Symptoms, 133

Fatigue, 133

In Vehicles, 133

Intercommunication System, 57

Inverse Square Law, Sound Level vs Distance, 33

Jack Hammer, 117

Kitchen, 51

Appliances (see individual listings), 51 $52-57,117-118$

Cabinets, Installation, 54

Doors, 52-55

Exhaust Fan, 52-55

Sound Conditioning the Room, 52-57

Knife Sharpener, 118

Landscaping, 100-101

Acoustical Properties, 100-101

Laundry Rooms, 101, 118

Appliances (see individual listings)

So und Conditioning, 101, 118

Lawn Tools, 13, 118-119
Edgers, 13, 118

Leaf Blowers, 118

Leaf Mulchers and Sweepers, 13, 118

Mowers, 13, 118

Choice of Quiet Unit, 118

Noise Hazard to Hearing, 13

Thatcher, 118

Light Leakage, Test for Noise Leaks, 105

Light Switches, 118

Lightning, Process of Noise Generation, 2

Line Sources, 34, 35

Localization of Sound, 104

Loudspeakers, 6, 32, 47

Loudness, 3, 6, 13

Equal Loudness Contours, 4-7

Growth with Duration, 7

Louvered Windows, Doors, 47, 73, 137

Masking Noise, 13, 14, 41-44, 119, 130

Acoustical Perfume, 41-44

Communication Problems, 14, 41

Effectiveness, 44

Hearing Effects, 8-14

Systems, 44,130

Magnetically-generated Noise, 114, 122

Mass Law, Weight vs Sound Insulation, 49

Mechanical Equipment, 16-17, 101, 118

Appliances, Criteria for Quiet, 16-17

Enclosures, 40, 127

Performance, 16

Reduction of Noise, 17-29

Motels, 135-138

Motors, 20, 70, 73, 78, 79, 118

Proper Installation in Appliances, 20

Heating Systems, 70, 78, 79

Machinery, 20

Plumbing Systems, 81

Motorboats, 8, 119, 120

Motorcycles, 8, 106, 119

Mufflers, 15, 28, 37, 119

Multipurpose Rooms in Schools, 131, 132

Music, 12, 41, 57, 119

Background Masking Effect, 41

Hearing Hazard Criteria, 12 
Music Rooms, 131

Nai1 Guns, 120

Needle Valves, 120

NIPTS, (Noise-Induced Permanent Threshold Shift), 8-9

Noise, 1

Airborne, Reduction of, 52, 54, 56

Broadband, 7, 41

Build up, 28, 29, 32, 40, 51, 54, 57

Causes, 1, 107-108

Characteristics of, 2,3

Control of Source, Path and Receiver, 16$29, \quad 102-104$

Definition, 1,7

Exposure, 40, 42

Damage to Hearing, $1,8-13$

Health Hazard, 8-15

Physiological Effects, 8-15, 41, 133

Stress-induced Diseases, 8

Field

Direct, 47-48

Reverberant, 47-48

Flanking Transmission, 47-48

Generation, 2, 17-23, 104

Impact, $17-18$

Leakage, Reduction, 28, 32, 56, 57, 105-107

Levels, Reduction of, 109

Loudness, 4-7, 13

Occupational, 14-15

Ordnances, 140-142

Outdoor, 35, 45, 106

Sources, 4

Structureborne, 2, 45-48

Subjective Responses to, 6-7

Noise Complaints, 107

Noise Control Act of 1972, 140

Noise Control Engineers, 2, 5, 17, 56, 88

Noise Leaks, 28, 32, 47, 57, 60, 68, 102, 104 Detection of, 104,105

Noise Problems and Remedies, 107-126, 140-142

Noise Radiation, $26,46,80$

Area Effect, 26, 46, 80

Noise Ratings, 16, 71

Noise Reduction, Basic Principles, Ch. 3, pp 16-44, 107-109

Noise Reduction Coefficient, NRC, 130
Noise Regulations, 124, 140-142

Federal, 140-142

State, 141-142

Loca $1,141-142$

Noise Sources, 4, 127

Indoors, 34, 45

Line Source, 34

Outdoors, 34, 45

Point Source, 33-34

Noise Surveys, 16, 17, 47, 98, 104, 136

Noise Warden, 142

Nystagmus, 15

Octave, 6

office, $120,127-132$

Acoustical Design, 129-130

Eq uipment, 120, 127-132

Furnishings, 129, 130, 132

Noise Sources, 127-129

Open Plan Design, 129-130

Privacy, 128-130

Selecting Quiet Location, 128-132

Open-plan Design 45, 57, 128-131

Apartments, 45, 57

Homes, 45, 57

Offices, 128

Schoo1, 129-131

OSHA, Occupationa1 Safety and Health Act, 140

Outdoor Noise, 35, 45, 86-87

Aircraft, 97

Amusement centers, parks, 86

Cìty, 138

Commercia 1,45

Industrial, 45

Neighborhood, 45, 86

Rural, 99

Shopping centers, 86, 98

Sport arenas, 30

Traffic, 86

Transportation, 110, 124

Partial Enclosures, 129-130

Path, Quieting, 33-40

Phons, 7

Pipes, 120

Enclosures of, 85

Expansion, 78

Flow Noise, 80

Isolation of, 23, 80

Layout to Minimize Noise Generation, 2126,82 
Noise Transmission, $80-81$

Sealing Against Noise, 28

Size, 82

Steam, 78

Turbulence, $78-80$

Valve Noise, 81

Vibration, 81

Water Hammer, 80-81, 125

Pitch, 3, 4, 6

Planning Against Noise, $16,17,51$

Playrooms, 57, 58

P1 umbing, 79-86, 120

Air Chambers, 81

Design of Quiet Systems, 18-23, 53, 81-85

Faucet Noise, 1, 81

Fixtures, Selection of Quiet, 83

Flow Noise, 81

Installation of, 21-22, 81

Noise Leakage, 28, 53, 81

Sinks and Drains, 53, 81

Toilets, 81

Turbulence in Flow, 21-23, 80

Point Source, 33-34

Polishers, 120

Power, 10, 15

Acoustic Power Leve1s, 3, 10, 15

Power Line Frequency, 5

Power Mower, 4, 8, 13, 106, 118

Power Tools, 13, 15, 120

Pressure Fluctuations, 1

Pressure Waves, 1,29

Privacy, 44, 58, 63, 80

Bathroom, 58, 80

Bedroom, 57

Offices, 44, 128

Schoo1, 137

Study, 57

Propagation of Sound, 29-34

In Air, Outdoors, 34

In Buildings, 34

Protecting the Receiver (Ear Protectors, etc.), 40-41

Pumps, 17, 23, 81, 120

Centrifugal Impeller Noise, 19, 79

Mounting, 23

Pressure Regulation, 20, 80

Pulsation Noise, 23, 78, 79
Sump, 120

Vibration Isolation, 23, 78, 81, 122

Water, 81,120

Punches, 127

Pure Tone, 7, 41

Quieting, criteria for

Apartments, 97-101

Appliances, 16, 17, 29

Automobiles, 133

Homes, 97-101

Motels, Hotels, 135-137

Offices, 127-128

Schools, 129-132

Tires, 134

Radiation of Sound, 26, 46, 80

Area Effect, 26, 46, 80

Prevention, 26

Processes, 26

Reduction, 26

Radiators, 78, 120

Hot Water, 78, 120

Steam, 78, 120

Valve Noise, 78

Radio, 13, 41, 58, 120, 133

Railroad Noise, 34, 97, 120, 135

Disturbance in Residential Area, 97, 9.8, 135

Propagation as a Line Source, 34, 35

Vibration, 120, 135

Rain Gutters and Spouts, 85, 121

Installation for Quiet Operation, 85

Noise from Turbulent Flow, 85

Reduction of Flow Noise, 85

Rarefaction, 1, 2, 29

Reaction Time, 14

Following Noise Exposure, 14-15

Recovery Time, 14

Recreation Rooms, 57-58

Noise Control, 57-58

Reflection of Sound, 31-32, 49

Refrigerators, 23, 52, 121

Compressor Noise, 47, 70

Reducing Noise Output, 23, 52

Resilient Attachments

Channels, 49, 58, 63, 65, 73, 92

Collars, 52-54, 72

Gaskets, 28, 52-59

Hangers, 23, 57, 65, 82 
Mounts, 52-54

Sleeves, 52-54, 57, 72

Resonance, 18, 47, 79

Reverberant Areas, 29, 32, 57

Reverberation Time, 32, 131-132

Ringing in Ears (Tinnitus), 11

Risers, 121

Duct, 121

Staircase, 69-70

Rock and Rol1, 8, 11-13, 121

Hazard to Hearing, 8, 11-13, 121

Safe Listening Levels, 121

Roofing, 121

Sound Deadening Metal Roofs, 121

Sound Insulating Capabilities, 121

Types, 121

Roofs, 91-95

Room Noise, 57-58, 107, 130-132

Rotor Tillers, 121

Sanders, 121

Saws, 121

Bench, 121

Chain, 13, 40, 106, 111, 121

Schools, 127-132

Noise Control, 130-132

Sewing Machines, 122

Vibration Isolating Mounting, 122

Shavers, Electrical, 122

Shock Absorbers, 18, 81

Shock Waves, 2, 10, 81, 122

Shower Stalls, 83-85, 122

Proper Installation, 83-85, 122

Sirens, 4, 14, 122

Audibility, 4, 14, 122

Emergency Use, 14, 122

Neighborhood Problems, 122

Skeet Shooting, 8

Sleep Interference, 8, 51, 58, 70, 77, 87, $135-136$

Snowmobiles, 12, 122

Noise Limits, 122
Protection for Operator, 40, 41, 122

Snubbers, 37

Solenoids, 122

Sources of Noise, 122

Sonic Booms, 2, 4, 122

Sound Absorption, 29-31, 34, 49

Confusion with. Insulation, 35, 50, 137

Sound Attenuation, 35

Sound Barriers, 32, 35-36, 40, 59-66, 130

Sound Diffraction, 32

Sound Directionality, 31

Sound Generation, 1-2, 7

Sound Insulation of

Attics, 91

Basements, 92

Craw1 Spaces, 9.2

Doors, 90

Enclosures, 40, 42

Roofs, 91

Wa1ls, 35, 91-92

Windows, 35,88

Sound Intensity, 2, 3, 6, 32

So und Leakage, $28,32,35,60$

Sound Levels, 4-6

A-weighted, 4-6, 102

B-weighted, 6

C-weighted, 6

Sound Level Meter, 3, 6, 12

Readings, 4

Weighting Networks, 4-6

Sound Power, 2, 3, 4-6, 15

Sound Pressure, 4-6

Sound Pressure Leve1, 2-4

Sound Propagation 1, 2, 29, 32, 33, 34

Sound Radiation Efficiency, 26, 46, 80

Sound Reflection, 31, 33, 34, 49, 70

Sound Silencers, 37

Sound Sources, 16

Extended Source, 33-34

Line Source, 33-34

Point Source, 33--34, 38 
Sound Surveys, Inspections, 16, 17, 47, 98, 104

Sound Transmission, 2, 35

Sound Transmission Class, STC, 61, 86

Sound Transmission Loss, 50, 60-62

Sound Velocity, 2

In Air, 2, 29

In Solids, 2

Sound Wave, 29-33

Direct, 31-32

Reflected, 31-32

Sounding Board Effect, 46-47, 53, 80, 82, 85

Soundproof, Construction, 40, 87, 127, 137

Source - Path - Receiver, 16, 29, 102, 104

Speaking Tubes, 75, 129, 137

Speech, 4, 41

Conversation Level, 4, 41, 60, 61, 102 $131-132$

Intelligibility, 61, 102-103

Recognition, 4, 102

Sounds, 11, 12, 15

Speed of Sound, 2, 29

In Air, 2

In Solids, 2

Spherical Wave Front, 33

Staircases, 50, 58, 122

Isolation of Footstep Noise, 50, 58

Reduction of Noise from Creaking, 69-70

Stapedius Reflex, 10

Startle Reflex, 15

State, Noise Regulations 140-142

Steam Heat Noise, 78

Steam VaIves, 122

Air Entrapment, 78-79

Noise Reduction, 1, 22

Stereo Sets, 46, 57

Stethoscopes, 44, 105, 106

Structureborne Sound 2, 17, 45, 49

Reduction, 38

Student Behavior, 132
Subjective Response to Noise, 6, 7

Sump Pumps, 123

Supersonic Airplanes, see Sonic Boom, 122

Swimming Pools, 50, 123, 137

Mote1s, 137

Residential Disturbance, 50, 122

Talk. Test, 136

Target Shooting, 12

Teaching-Learning Process, 130-132

Telephone, 12, 123, 127

Television, 12-13, 51, 60, 123

Antenna, 123

Electronic Noise, 123

Temporary Threshold Shift (TTS), 8-9

Thermal Vibration, 2

Thermostats, 79, 123

Threshold, of Hearing, 3-4, 7

of Pain, 3-4

Threshold Shift in Hearing, 8-11

Thunder, 1, 2, 4, 6, 30, 124

Noise Generation Process, 1-2

Tickle Sensation, 11

Tinnitus, 11

Tire Noise, 14, 124, 134

Quiet Tread Design, 134

Toilets, 81,124

Tools, 124

Toys, 124

Hearing Hazard, 124

Traffic Noise, 4, 14-15, 87, 124, 127

Effects on Driver Safety, 14-15

Emergency Sirens and Signals, 14

Reaction Time of Driver, 15

Startle Effects, 15

Transformers, 79, 124

Transmission of Airborne $\&$ Structureborne Noise, 35, 38, 45, 49, 106 
Transmission Path, 16, 29, 30, 33, 47, 74, 105,106

Trash Cans, Collection, 29

Transportation Noise, 124

Aircraft, 87,110

Automobiles, 132-136

Buses, 111

Motorcycles, 119

Trains, 124, 135

Trucks, 124

Turbulence, $2,5,18,19,21,23,37,71,78$, 126

Air, 18, 19, 21, 23, 126

Water, 18, 19, 21, 23, 78, 126

Typewriters, 17, 18, 127

U. S. Environmental Protection Agency (EPA) $140-142$

Vacuum Cleaner, 4, 13, 105, 124-125

Hose Noise, 29

Valves, 125

Flow Noise; 125

Pressure-reducing, 48, 122, 125

Types for Quiet Operation, 125

Water Hammer, 125

Ventilation System Noise, 20, 59, 70-71, 125, 127,133

Vibration Attenuation, 23-28

Vibration Control, 23-25

Vibration Damping Materials, 26-28 Application to Surfaces, 27-28, 54

Vibration Detectors, 104

Vibration Isolation, 23-25, 38-40, 52-54, 73, 80

Vibrationa1 Resonance, 72, 134

Voice Projection, 132

Wa11s

Acoustical Treatment, 34, 35

Construction, 49

Double, 49

Exterior, 45, 60-65, 86, 91, 136

Interior, 45, 49, 59, 60-65

Isolation, 45, 60-65

Noise Leaks, 61, 62, 86

Sound Insulation, 60-65, 98

Transmission of Sound, 2 Airborne, 45-48

Structureborne, 45-48
Wa11 Treatments

Increase sound absorption, 34, 35

Reduce sound transmission, 60

Washing Machines, 17, 23, 52, 125

Noise Reduction, 23, 52

Vibration Isolation, 23, 52

Water Coolers, 125

Water Noises, 78, 125

Turbulent flow in pipes, 78

Valve noise, 81, 125

Water hammer elimination, 81, 125

Water Pressure, 20

Water Skiing, 8

Wave Form, 2

Wave Front, 31, 33

Cylindrica1, 33

Spherical, 33

Wave Length of Sound, 29

Weatherstripping, 91, 92

Weighting Networks, Sound Level Meters, 4-6

Wheel Balance, 20-21, 134

Whisper, 4

Whistling Noises, 6, 7, 31, 126

Dampers and Ductwork, 77, 112, 115

Valves, 80-81, 125

Wind Noise, 7, 133

Window, 35, 125-126

Automobile, 133

Leakage of Sound, 35, 125-126

Noise intrusion, 86-88

Rattling, 125

Sealing against noise, 88-90

Sound Insulation, 88

Windshield Wiper Noise, 134-135

Windstream Noise, 133, 135

Work Scheduling to Minimize Hearing Damage Neighborhood Disturbance, 40-41 


\begin{tabular}{|c|c|c|c|}
\hline $\begin{array}{l}\text { U.S. DEPT. OF COMM. } \\
\text { BIBLIOGRAPHIC DATA } \\
\text { SHEET }\end{array}$ & $\begin{array}{l}\text { 1. PUBLICATION OR REPORT NO. } \\
\text { NBS HB- } 119\end{array}$ & $\begin{array}{l}\text { 2. Gov't Accession } \\
\text { No. }\end{array}$ & 3. Recipient's Accession No. \\
\hline \multicolumn{3}{|l|}{ 4. TITLE AND SUBTITLE } & $\begin{array}{l}\text { 5. Publication Date } \\
\text { Ju1y } 1976\end{array}$ \\
\hline \multicolumn{3}{|c|}{ Quieting: A Practical Guide to Noise Control } & 6. Performing Organization Code \\
\hline \multicolumn{3}{|c|}{$\begin{array}{l}\text { 7. AUTHOR(S) } \\
\text { Raymond D. Berendt, Edith L. R. Corliss, and Morris S. Ojalvo* }\end{array}$} & 8. Performing Organ. Report No. \\
\hline \multirow{2}{*}{\multicolumn{3}{|c|}{$\begin{array}{l}\text { 9. PERFORMING ORGANIZATION NAME AND ADDRESS } \\
\text { NATIONAL BUREAU OF STANDARDS } \\
\text { DEPARTMENT OF COMMERCE } \\
\text { WASHINGTON, D.C. } 20234\end{array}$}} & $\begin{array}{l}\text { 10. Project/Task/Work Unit No. } \\
2130116\end{array}$ \\
\hline & & & 11. Contract/Grant No. \\
\hline \multirow{2}{*}{\multicolumn{3}{|c|}{ 12. Sponsoring Organization Name and Complete Address (Street, City, State, ZIP) }} & $\begin{array}{l}\text { 13. Type of Report \& Period } \\
\text { Covered } \\
\text { Final }\end{array}$ \\
\hline & & & 14. Sponsoring Agency Code \\
\hline \multicolumn{4}{|c|}{$\begin{array}{l}\text { 15. SUPPLEMENTARY NOTES } \\
\qquad \begin{array}{l}* \text { Guest worker from the National Science Foundation } \\
\text { Library of Congress Catalog Card Number: 76-5500. }\end{array}\end{array}$} \\
\hline
\end{tabular}

16. ABSTRACT (A 200-word or less factual summary of most significant in formation. If document includes a significant bibliography or literature survey, mention it here.)

This guide offers practical solutions for ordinary noise problems that a person is likely to meet. The discussion describes the ways in which sounds are generated, travel to the listener, and affect his hearing and well-being. Recommendations are given for controlling noise at the source and along its path of travel, and for protecting the listener. The guide instructs the reader by way of "Warning Signs" on how to determine whether he is being subjected in his environment to prolonged noise exposures that may prove hazardous to his hearing. Remedies are given for noise problems that a person is likely to find in his home, at work and at school, while traveling, and in the growth and development of his community. The remedies include noise prevention techniques and selection of quiet alternatives to existing noise sources. General principles for selecting quiet appliances are given. Ways of searching for the sources of noise and for deternining the paths over which they travel to the listener are described. A detailed index is given for individual noise sources describing specific solutions to the problems they present. General ways of looking for inherently quiet homes and travel accomodations are described。 In a final chapter, there are suggestions for enlisting community help where large external sources of noise must be quieted, such as those arising from public utilities and public transportation.

\section{KEY WORDS (six to twelve entries; alphabetical order; capitalize only the first letter of the first key word unless a proper} name; separated by semicolons) Airborne and structure-borne sounds; aircraft noise intrusion; annoyance; appliance noise; health and hearing hazards; household noise; legal and community action; loudness; noise control and abatement; traffic noise.
18. AVAILABILITY
X Unlimited

For Official Distribution. Do Not Release to NTIS

X Order From Sup. of Doc., U.S. Government Printing Office Washington, D.C. 20402, SD Cat. No. C13.11:119

Order From National Technical Information Service (NTIS) Springfield, Virginia 22151

\begin{tabular}{|l|c|}
\hline $\begin{array}{l}\text { 19. SECURITY CLASS } \\
\text { (THIS REPURT) }\end{array}$ & $\begin{array}{c}\text { 21. NO. OF PAGES } \\
163\end{array}$ \\
$\begin{array}{l}\text { UNCL ASSIFIED } \\
\text { (THIS PAGE) } \\
\text { UNCLASSIFIED }\end{array}$ & $\begin{array}{r}\text { 22. Price } \\
\$ 5.10\end{array}$ \\
\hline
\end{tabular}




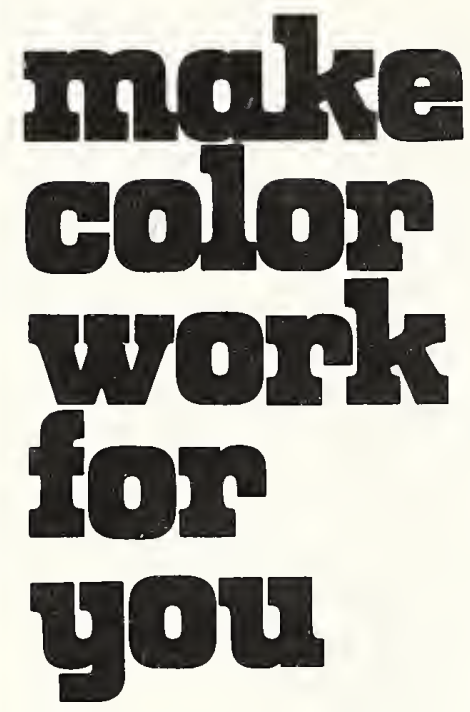

Color in our Daily Lives, a new consumer booklet from the National Bureau of Standards, takes the reader step by step through the fundamental principles of color and light, families of color, influence of colors upon other colors, and color harmony. This fullcolor, 32-page illustrated booklet highlights practical applications of color, including:

- Your personal color plan.

- Your color environment.

- Color plans for the home.

- Using color to drama. tize or to hide.

- Color and illumination.

- Experimenting with color.

This new basic guide can serve as your handbook in helping you make decisions about how to use color in your life and make it work for you. Order Color in Our Daily Lives prepaid for $\$ 1.70$ from the Superintendent of Documents, U.S. Government Printing Office, Washington, D.C. 20402. Use SD Catalog No. C13.53:6. 





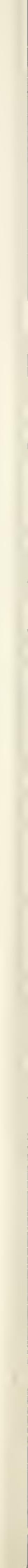





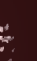

